Florida International University FIU Digital Commons

7-20-2009

\title{
Risk factors of type 2 diabetes and cardiovascular diseases among Jamaican adolescents
}

Sheila C. Barrett

Florida International University

DOI: $10.25148 /$ etd.FI14050445

Follow this and additional works at: https://digitalcommons.fiu.edu/etd

Part of the Dietetics and Clinical Nutrition Commons

\section{Recommended Citation}

Barrett, Sheila C., "Risk factors of type 2 diabetes and cardiovascular diseases among Jamaican adolescents" (2009). FIU Electronic Theses and Dissertations. 1413.

https://digitalcommons.fiu.edu/etd/1413

This work is brought to you for free and open access by the University Graduate School at FIU Digital Commons. It has been accepted for inclusion in FIU Electronic Theses and Dissertations by an authorized administrator of FIU Digital Commons. For more information, please contact dcc@fiu.edu. 
FLORIDA INTERNATIONAL UNIVERSITY

Miami, Florida

RISK FACTORS OF TYPE 2 DIABETES AND CARDIOVASCULAR DISEASES AMONG JAMAICAN ADOLESCENTS

A dissertation submitted in partial fulfillment of the

requirements for the degree of

DOCTOR OF PHILOSOPHY

in

DIETETICS AND NUTRITION

by

Sheila C. Barrett 
To: Dean Fernando M. Treviño

R.Stempel College of Public Health and Social Work

This dissertation, written by Sheila C. Barrett, and entitled Risk Factors of Type 2 Diabetes and Cardiovascular Diseases among Jamaican Adolescents, having been approved in respect to style and intellectual content, is referred to you for judgment.

We have read this dissertation and recommend that it be approved.

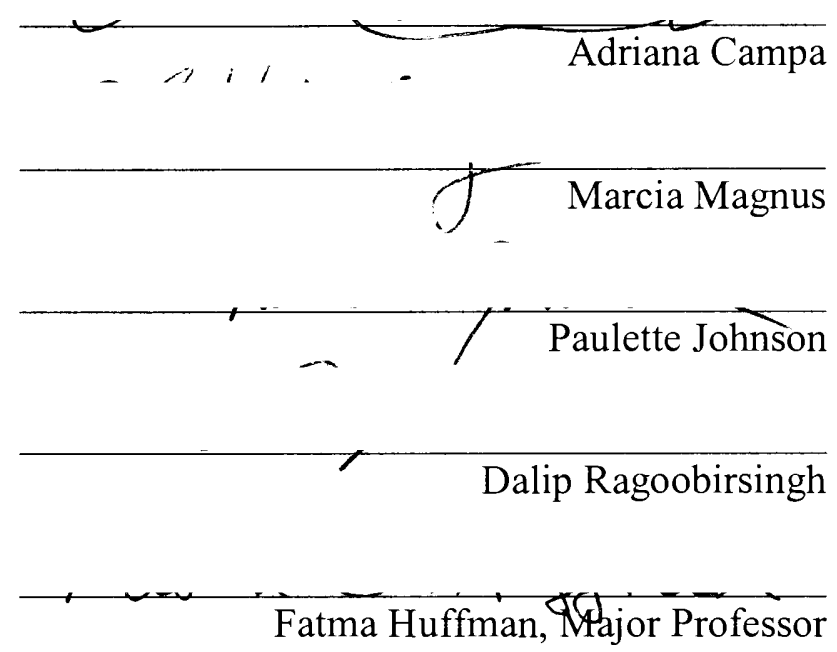

Date of Defense: July 20, 2009

The dissertation of Sheila C. Barrett is approved.

Dean Fernando M. Treviño R.Stempel College of Public Health and Social Work

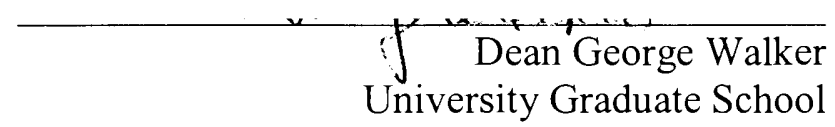

Florida International University, 2009 


\section{DEDICATION}

The study is dedicated to the memory of my mother, Olive Barrett who died of diabetes complications, my father, Ivan Barrett who later followed with ischemic heart disease. The pain of losing my parents prompted this research to help bring awareness to others. 


\section{ACKNOWLEDGMENTS}

I wish to thank my committee members for their support during the preparation and compilation of this study. I appreciate their time, effort and guidance throughout the process. Special gratitude to Dr. Paulette Johnson for her help and patience with the statistical analyses, Dr. Adriana Campa, for her suggestions in methodology, Dr. Marcia Magnus and Dr. Dalip Ragoobirsingh for their scrutiny of the proposal. To my major professor, Dr. Fatma Huffman, thank you for your faith in my ability to complete this study. Her gentleness, encouragement, guidance, financial planning and assistance helped me to meet the deadlines and requirements for completion of this study. Thanks to the University Graduate School, FIU for awarding me the Dissertation Year Fellowship during my final year of the study.

Special thanks to the workers of the Heart Foundation of Jamaica, the Diabetes Association of Jamaica, and the Central Medical Laboratory, who performed the blood analyses. To the Jamaican Ministry of Education and Ministry of Health officials who sanctioned the collection of data. To the principals and contact persons in all ten schools who accommodated us for the data collection. To the study subjects who willingly participated and made this project possible.

To my family members here in the USA and in Jamaica who supported me during this challenging process. I am extremely grateful to my sisters, Cheryl and Heather Barrett in Jamaica who worked tirelessly to organize this study for me and made followup calls to ensure the smooth success of the project. To Francine White who assisted me throughout the data collection and to my nephews who took time out from their school activities to accompany me on data collection days. Your help is greatly appreciated. 


\section{ABSTRACT OF THE DISSERTATION \\ RISK FACTORS OF TYPE 2 DIABETES AND CARDIOVASCULAR DISEASES AMONG JAMAICAN ADOLESCENTS}

by

Sheila C. Barrett

Florida International University, 2009

Miami, Florida

Professor Fatma Huffman, Major Professor

Purpose: The purpose of the study was to examine Jamaican adolescents in a school setting, for risk factors of type 2 diabetes mellitus (T2DM) and cardiovascular diseases (CVDs).

Methods: A descriptive epidemiological cross-sectional study of 276 Jamaican

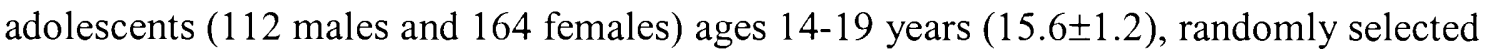
from grades 9-12 from ten high schools on the island. Thirteen risk factors were examined. Risk factors were compared with BMI levels and demographics. A sub-study validated finger prick testing of fasting blood glucose, total cholesterol, and $\mathrm{HbAlc}$ versus venous testing in 59 subjects.

Results: Prevalence of overweight was $33.0 \%(n=91)$ with mean BMI of $23.74 \pm 7.74$. Approximately $66.7 \%$ of subjects reported $\geq 3$ risk factors. The number of T2DM and CVDs risk factors increased for subjects with BMI above 25. One third of the overweight subjects were classified with the metabolic syndrome. High BMI was associated with high waist circumference $(\mathrm{r}=.767, \mathrm{p}<.01)$, high waist-to-hip ratio 
$(\mathrm{r}=.180, \mathrm{p}<.01)$, presence of Acanthosis Nigricans $(\mathrm{r}=.657, \mathrm{p}<.01)$, high total cholesterol $(\mathrm{r}=.158, \mathrm{p}<.01)$, family history of T2DM $(\mathrm{r}=.157, \mathrm{p}<.01)$, and hypertension $(\mathrm{r}=.422, \mathrm{p}<.01)$. Regression analyses significantly predicted gender and physical activity $(\mathrm{p}<.001)$, and total number of risk factors for T2DM and CVDs $(\mathrm{p}<.001)$. Paired samples t-tests revealed no significant differences between methods of testing for TC and HbAlc $(\mathrm{p}<.01)$ but not for FBG $(\mathrm{p}>.05)$. Percentage bias for the methods of blood testing met the reference standards for fasting blood glucose but not for total cholesterol and $\mathrm{HbAlc}$. Bland Altman tests of agreement between the two methods indicated good agreement for all three tests.

Conclusion: Jamaican adolescents are at high risk for T2DM and CVDs as seen in other study populations. Effective programs to prevent T2DM and CVDs are needed. Family history of diseases, anthropometric measures, and gender identified more subjects at risk than did the biochemical measures. Comparison between finger prick and venous blood methods suggested that finger prick is an adequate method to screen for risk factors in children and adolescents. 
I.

INTRODUCTION

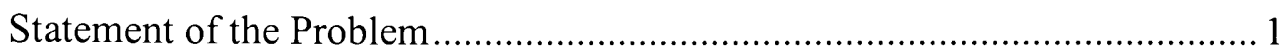

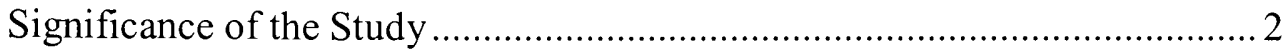

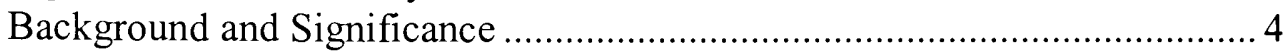

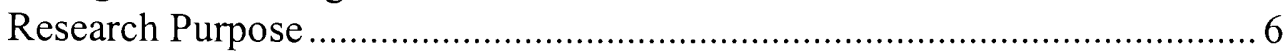



Research Questions, Objectives and Hypotheses ......................................... 9

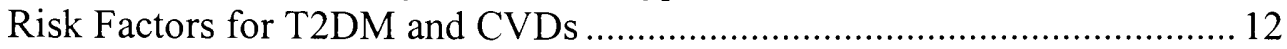

T2DM and Obesity among Adolescents ................................................. 16

Obesity and T2DM in Jamaican Adolescents ........................................... 17

Relationship of Obesity to T2DM and CVDs............................................. 19

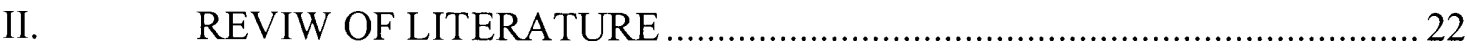

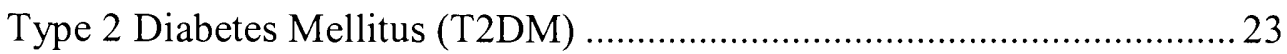

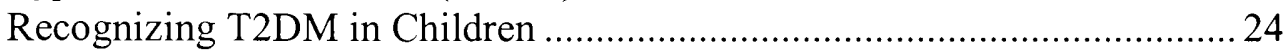

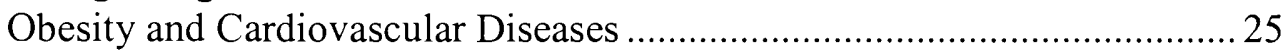

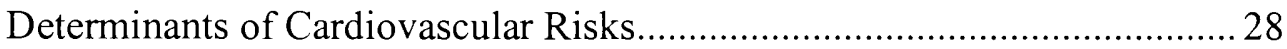

Definition of Overweight and Obesity......................................................29

Prevalence of Overweight and Obesity...................................................... 33

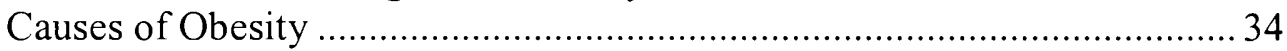

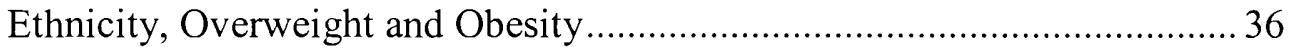

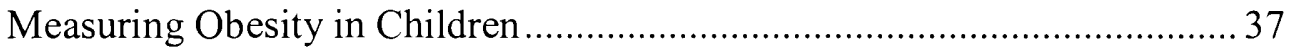

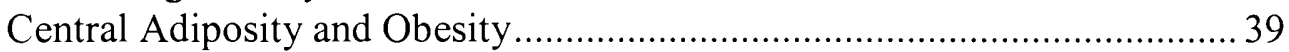

Clustering of Risk Factors for T2DM and CVDs ...................................... 41

Health, Social, and Economic Costs of Obesity ......................................... 42

Acanthosis Nigricans, Obesity, Type 2 Diabetes,

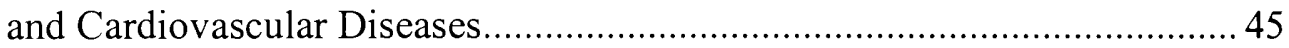

Obesity and Physical Activity and Relationship to

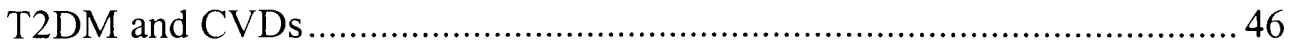

Obesity, T2DM, CVDs and Food Intake, ................................................... 47

Comparison of Finger Prick versus Venous Blood Testing........................... 49

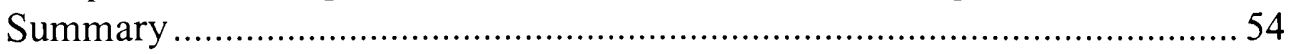

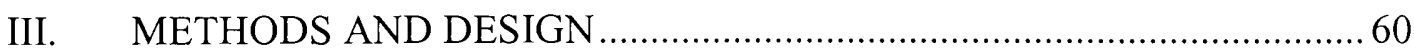

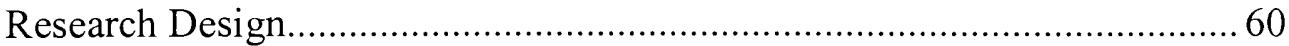

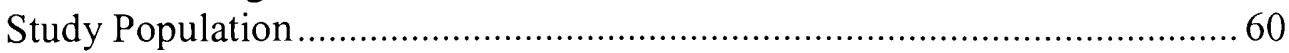

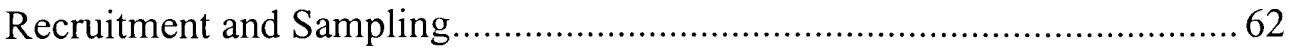

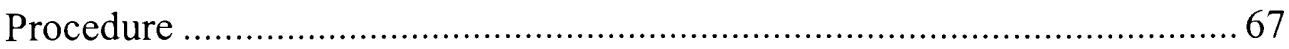


Pilot Testing of Instruments..................................................................... 70

Data Collection and Description of Instruments.......................................... 70

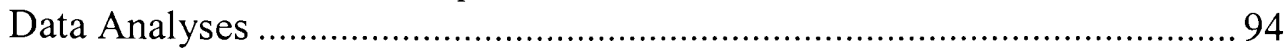

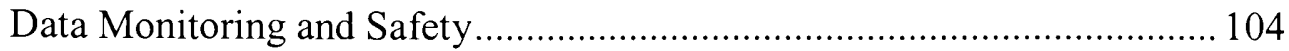

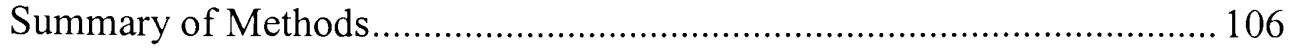

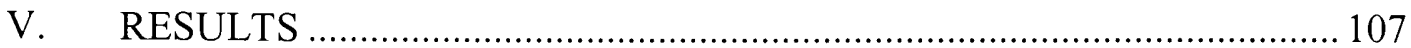

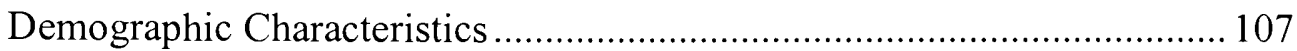

General Description of the Risk Factors ..................................................... 110

Total Number of Risk Factors for T2DM and CVDs ................................. 117

Number of Risk Factors and the Metabolic Syndrome................................ 119

Results Relating to Hypotheses Testing .................................................... 120

Validation of Finger Prick Method of Blood Testing

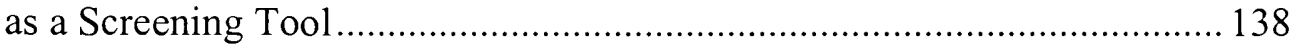

Summary of Validation Study .............................................................. 150

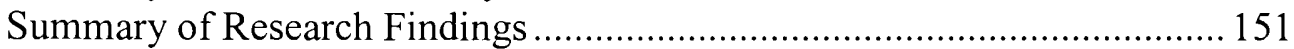

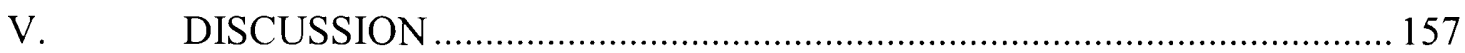

General Description of Study Subjects ................................................... 157

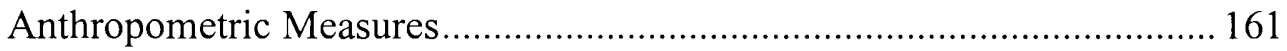

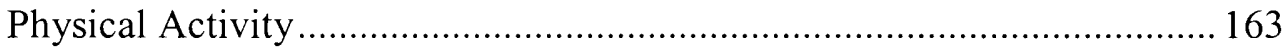

Presence of Acanthosis Nigricans............................................................ 166

Socio Demographic Characteristics and Risk Factors

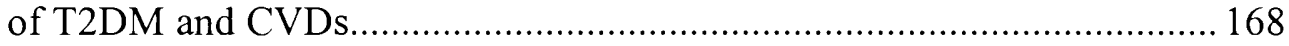

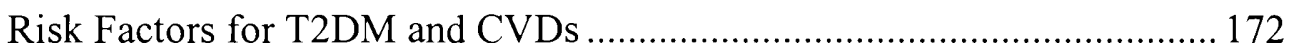

Waist Circumference and Waist-to-Hip-Ratio......................................... 176

BMI and Family History of Obesity ................................................. 178

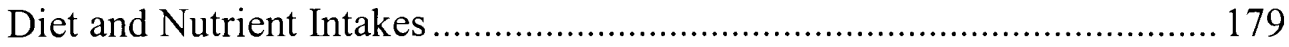

Comparison of Finger Prick vs. Venous Blood ......................................... 183

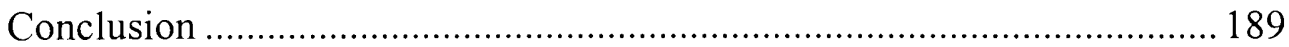

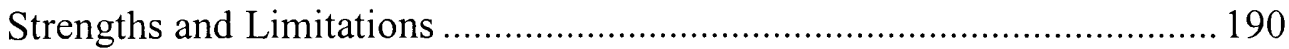

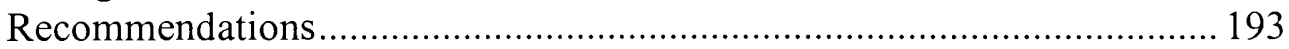

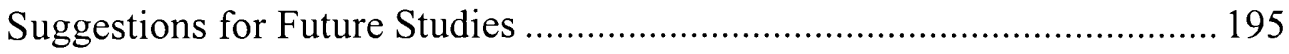



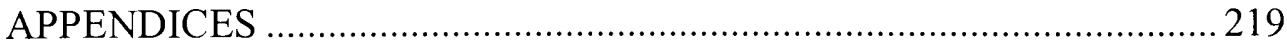

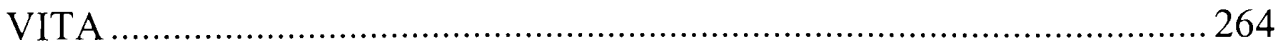




\section{LIST OF TABLES}

\section{TABLE}

1. Research Questions, Objectives and Hypotheses 9

2. WHO Classification of Obesity and Risk of Co-Morbidities 31



4. Waist Circumference and Obesity-Associated Metabolic Complications.

5. Summary of Literature on T2DM and CVDs among Adolescents 55

6. Breakdown of Population by Regions, Parishes, School Type and Location.

7. Statistical Analyses 99

8. Demographic Characteristics of the Study Population 109

9. Anthropometric Measures of Study Subjects

10. Biochemical Risk Factors of T2DM and CVDs

11. Physical Activity, Family History of T2DM, CVDs, and Obesity

12. Presence and Severity of Acanthosis Nigricans 116

13. Number of Risk factors for T2DM and CVDs 118

14. Relationships among Gender, Ethnicity, Place of Residence, and Income and the Risk Factors of T2DM and CVDs

15. Cross-tabulations and Odds Ratios of Individual Risk Factors with BMI Classifications

16. Logistic Regression Analysis of BMI with Risk Factors for T2DM and CVDs.

17. Relationship between WC and WHR to Risk Factors of T2DM and CVDs .... 130

18. Correlation Statistics of Subjects' and Parents' BMI 


\section{LIST OF TABLES}

19. Caloric and Macronutrient Intakes Estimated by 24-hour Recall..................... 136

20. Demographic Characteristics of Sub-Study Sample .......................................139

21. Comparison of Sample to Sub-Study Sample: Blood Measures ....................... 140

22. Frequency and Correlations of FBG, TC and HbA1c................................. 141

23. Paired Samples t-test for FBG, TC, and HbAlc .......................................... 142

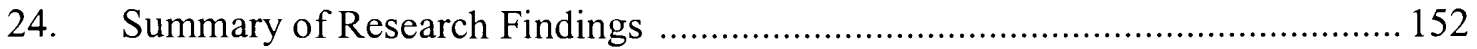




\section{LIST OF FIGURES}

FIGURE

PAGE

1. Comparison of Overweight and Normal Weight Subjects by Number of Reported Risk Factors of T2DM and CVDs

2. Comparison of Subjects' and Parents' BMI 132

3. Comparison of Finger Prick and Venous Fasting Blood Glucose

4. Comparison of Fasting Blood Glucose by Finger Prick and Venous Method

5. Bland Altman Plot for Finger Prick and Venous Method FBG 145

6. Comparison of Finger Prick and Venous Method TC 146

7. Bland Altman Plot for Finger Prick and Venous TC 147

8. Comparison of Finger Prick and Venous $\mathrm{HbAlc}$ 148

9. Bland Altman Plot for Finger Prick and Venous HbAlc 


\section{GLOSSARY}

Acanthosis Nigricans

Body Mass Index

Fasting Blood Glucose

Fasting Plasma Glucose

Gylcated Hemoglobin

Hypertension

Insulin Resistance

Macronutrients

Metabolic Syndrome

Obesity

Overweight
A skin disorder manifested by velvety light-brown to black marks around the neck, axilla, knuckles, elbows and is associated with obesity and insulin resistance.

A crude estimate of body fat calculated by dividing a person's weight by their height (weight $(\mathrm{kg}) /$ height $\left(\mathrm{m}^{2}\right)$

Measurement of the amount of sugar in whole blood and is taken at least 8 hours after eating, useful in diagnosing diabetes.

Measurement of the amount of sugar in plasma and is taken at least 8 hours after eating, the preferred glucose measurement for the diagnosis of diabetes.

A form of hemoglobin $(\mathrm{HbAlc})$ that remains bound to the glucose molecule used to identify the average plasma glucose concentration over a prolonged period (120 days) lifespan of the RBCs.

Elevated high blood pressure defined by both above normal levels of diastolic and systolic blood pressure.

A medical condition in which the body cells do not respond effectively to effects of insulin; a key feature of type 2 diabetes.

Dietary fat, protein and carbohydrates, referred to as "macro" due to the large amounts needed by the body.

A combination of associated cardiovascular risk factors to include central adiposity, high fasting plasma glucose, hypertension, high levels of LDL-C and low HDL-C cholesterol. Presence of any three components constitutes the metabolic syndrome.

Is a medical condition associated with excessive accumulation of body fat. Determined by BMI> $30 \mathrm{~kg} / \mathrm{m}^{2}$

A state of health with having more body fat than is considered healthy $(20 \%$ above normal body weight for height). 
Physical Activity

Type 2 Diabetes

Total Cholesterol

Waist Circumference

Waist-to-Hip Ratio
Activity that is intended for improving or maintaining physical fitness and health.

A health condition formerly associated with age, but now found in younger age group. Associated with obesity, and insulin resistance, and is more common in ethnic minority groups.

The combination of all measurement of all types of cholesterol in the blood.

A measurement of the waist taken around the abdomen. Fatty deposits around the waist indicate increased health risks. Waist circumference of $102 \mathrm{~cm}$ (40 inches) in men and $88 \mathrm{~cm}$ ( 35 inches) in women suggest increased health risks.

Waist measurement divided by the hip circumference. Healthy Waist-to-hip-ratio is $<1.0$ for men and $<.8$ for women. 


\section{LIST OF ABREVIATIONS}

AN

ACSM

AMDR

BMI

BMR

CDC

CVD

ECG

FAO

GDP

HDL-C

IOTF

IR

LDL-C

MET

METS

NCEP

NHES

NHANES

PA

PAQ-C

SES
Acanthosis Nigricans

American College of Sports Medicine

Acceptable Macronutrient Distribution Ranges

Body Mass Index

Basal Metabolic Rate

Center for Disease Control and Prevention

Cardiovascular Disease

Electrocardiogram

Food and Agricultural Organization

Gross Domestic Product

High Density Lipoprotein Cholesterol

International Obesity Task Force

Insulin Resistance

Low density Lipoprotein Cholesterol

Metabolic Equivalent

Metabolic Syndrome

National Cholesterol Education Program

National Health Examination Survey

National Health and Nutrition Examination Survey

Physical Activity

Physical Activity Questionnaire for Children

Socio economic status 
Total Cholesterol

TG

Triglycerides

T2DM

Type 2 diabetes mellitus

USDHHS

United States Department of Health and Human Services

WHO

World Health Organization 


\section{CHAPTER I \\ INTRODUCTION}

\section{Statement of the Problem}

The prevalence of Type 2 diabetes mellitus (T2DM) in children and adolescents with its complications and premature mortality is increasing and presents a major public health challenge. Type 2 diabetes mellitus is among the cluster of cardiovascular diseases (CVDs) risk factors such as obesity, hypertension, and dyslipidemia, known as the "Metabolic Syndrome" (METS) and is considered the seventh leading cause of death in the developing world (Sacks, Bruns, \& Goldstein et al.,2005). Once thought to relate only to adults, T2DM is now increasingly found in children and adolescents (Daniels, 2006; Choudbury, 2005, Reinehr, 2005).

Health conditions such as heart attack and strokes are chronic, start in childhood, and take decades to present as overt conditions (Daniels, 2006; Wyatt, Winter, \& Dubbert et al., 2006). Being obese during childhood, adolescence and young adulthood may accelerate these conditions (Daniels, 2006; Choudbury, 2005).

Most of the data on childhood obesity and related health complications were collected in the developed countries. As lifestyles change in the developing world to pattern that of the developed countries, there is need for research among this population. This study investigated the prevalence of risk factors for T2DM and CVDs among Jamaican adolescents ages 14-19 years. The study was undertaken to help raise awareness of the presence of risk factors such as overweight and obesity, family history of chronic nutrition-related diseases, decreased physical activity, and poor dietary intakes among this population. A secondary aim was to validate a screening method for testing 
blood values of fasting blood glucose (FBG), total cholesterol (TC), and glycated hemoglobin $(\mathrm{HbAlc})$ which can be used in a school setting, with easier testing for, and faster feedback than traditional clinical setting.

\section{Significance of the Study}

The study is significant because with data it will be possible to track trends, provide the rationale for the implementation of programs that can help in the prevention of chronic diseases, help to provide a healthier future generation for the workforce, save on healthcare costs, and provide a better quality of life for Jamaicans.

The growing prevalence of T2DM and CVDs is associated with the growing rates of obesity. Type 2 diabetes mellitus in minority American children over ten years old now accounts for $8-45 \%$ of new cases of diabetes (ADA, 2005). Approximately 60 percent of overweight American children also have hypertension, hyperinsulinemia, and/or hyperlipidemia (Freedman, Dietz, Srinivasan et al, 1999). The prevalence rate of obesity (BMI exceeding the $95^{\text {th }}$ percentile) among US children ages $12-19$ years has increased by $10 \%$ over a twenty year period. That is, adolescent obesity has increased from $5 \%$ in 1980 to $15.5 \%$ in 2000 in the USA (Bloomgarden, 2004). Therefore, the proposed study is significant for a number of reasons; it is an unprecedented assessment of risk factors of T2DM and CVDs among Jamaican adolescents.

There is no documented research on T2DM in Jamaican children and adolescents despite the current interest and prevalence shown in children from other countries. Screening for T2DM is necessary since the onset of T2DM occurs 4-7 years before clinical diagnoses (Sacks et al., 2005). Second, research on childhood obesity in the 
developing world is sparse and this will add to the body of literature on childhood obesity in the developing world. The rate of obesity is expected to pattern that of the US since the diets have become more similar to US foods with high levels of imported refined grains, oils, and other high energy-dense foods. Third, it will provide baseline data so that trends can be examined retrospectively. In addition, this study will help to rejuvenate interest in preventive health care that can be accessed more readily and economically at the school level without overburdening the already strapped healthcare system.

In the 1960s-70s when the health concerns were that of childhood infectious diseases such as measles, smallpox, chicken pox and tuberculosis, the Jamaican government took more active role in the health of school children. Children were targeted and immunized within the school system. Now that the focus has shifted to noncommunicable diseases such as T2DM and CVDs, there is need for government based primary prevention programs that target school children for these health conditions.

Reduction of health care costs is important to countries such as Jamaica with low Gross National Product (GNP) and which are overburdened with high healthcare costs. Total health expenditure in Jamaica was J\$9.1 billion in 1993-1994 and represented up to $8.9 \%$ of the Gross Domestic Product (GDP) at that time (Lalta, 1995). Due to massive devaluation of the Jamaican currency, Jamaica's GDP was US\$8.9 billion in 2004 with GDP per capita income of US\$3,350.00 (Jamaica Facts and Figures, 2005). More recent reports showed that Jamaica spends approximately $6 \%$ of GDP on the treatment of diabetes and hypertension (PAHO, 2008). Early screening for risk factors of T2DM and CVDs is needed to help reduce health care costs. 
The numbers of persons without health insurance in Jamaica is not documented but the percentage without insurance is expected to be even higher than in a developed country. Up to $11.4 \%$ of children in the USA had no health insurance in 2003 ("Center on Budget and Policy Priorities," 2004). In Jamaica, about 15\% of persons who have health insurance purchased private health insurance (Lalta, 1995). Many persons pay out of pocket for health care with approximately $54 \%$ doing so in $1993-94$. It is likely that even if parents are aware of the risks of overweight and obesity, they are unable to solicit proper healthcare with no medical insurance to help offset the costs.

US based research indicated strong associations with being overweight during childhood and adolescence with insulin resistance (IR), dyslipidemia, and elevated blood pressure in young adulthood (Gungor \& Arslanian, 2002; Steinberger, Moran, Hong et al., 2001; \& Daniels, 2001; Daniels, 2006; Choudbury, 2005; Reinehr, 2005). This epidemiological study identified adolescents at risk to T2DM and CVDs. It is an unprecedented study which examined Jamaican adolescents on thirteen risk factors for T2DM and CVDs. Identification of these risk factors can help plan early preventive programs for this population.

\section{Background and Significance}

Recent research shows an increasing global prevalence of T2DM in children and adolescents due to a concurrent rise in the prevalence of obesity (Reinehr, 2005). Further, childhood obesity is closely associated with IR in early adulthood (Steinberger, et al., 2001). Other factors such as ethnicity, gender and family history of disease have been associated with the development of T2DM. 
A study of 167 obese US adolescents revealed a prevalence of $4 \%$ of T2DM, all cases reported were among Hispanics and Blacks (Sinha, Fisch, \& Teague et al, 2002). Earlier studies (Rosenbloom, Joe, \& Young et al., 1999) indicated that girls were 1.7 times more likely to develop T2DM than boys. Research shows that over two-thirds of children with T2DM have at least one parent with T2DM (ADA, 2000). Other studies also revealed higher prevalence of T2DM among females than males and among minority groups compared to Caucasians (Rosenbloom, et. al, 1999, McKnight-Menci, Sababu, \& Kelly et al, 2005).

Type 2 diabetes mellitus is found in up to $45 \%$ of new cases of diabetes in children and adolescents at health centers in the US, which serve large numbers of African American, Mexican American and Native American youths (ADA, 2000, ADA, 2005, Bavdekar, Yajnik, \& Fall et al, 1999; Berry, Sheshan, \& Heschel et al, 2004). Detection of the early signs of T2DM and CVDs is needed to help in the prevention of these and other related medical conditions. Type 2 diabetes mellitus in children and adolescents relates to obesity and features of the metabolic syndrome (METS) such as hyperinsulemia, hypertension, \& dyslipidemia. Other associated indicators include polycystic ovarian syndrome (PCOS) and Acanthosis Nigricans (AN). It is difficult to diagnose T2DM in adolescents since there are few symptoms such as mild polyuria and polydipsia which tend to appear around mid to late puberty (Reinehr, 2005). Screening is recommended for children over age 10 years and at any age if the child is overweight and has two other risk factors. Other risk factors include family history of T2DM, race/ethnicity, and signs of IR. The presence of Acanthosis Nigricans (AN) (a dark line around the neck) is a sign of IR and when present, it is easily detectable and needs to be 
considered as an additional risk factor. It is further recommended that screening for T2DM be carried out every two years (Sacks, al., 2002).

Cardiovascular diseases are manifested in the middle or older years. However, the development usually starts in the earlier years and progresses to clinically significant lesions in young adulthood (Gungor, \& Arslanian, 2005; Steinberger \& Daniels, 2003). Research has shown that the fatty streaks associated with atherosclerosis were found in the aortas of children as young as three years old (McGillis-Bindler, \& Bruya, 2000). Other studies indicated that about $60 \%$ of overweight American children also had hypertension, hyperinsulinemia, and/or hyperlipidemia (Freedman, et al., 1999).

This research was conducted on a group of Jamaican adolescents since the epidemiological transition is underway in Jamaica among adults. The prevalence rate of obesity among Jamaican women was $25 \%$ and twice that of the men. Up to $51 \%$ of deaths in the Caribbean region during the late 1990s related to diabetes, cancer, and cardiovascular diseases [The Caribbean Commission on Health and Development (CCHD) 2005 Report]. The increase in obesity among the adult population was attributed to excessive consumption of foods, wrong food choices, and low physical activity. Similar trends were expected among Jamaican adolescents. Information on Jamaican adolescents is needed to estimate the future barriers to addressing lifestyle diseases for this country of limited health resources.

\section{Research Purpose}

Testing for risk factors of T2DM in children and adolescents is necessary since earlier research indicated that "the presence of T2DM in one-third of the minority 
children with T2DM, and the majority of Caucasian children with T2DM was detected in the absence of symptoms" (Reinehr, 2005). The Centers for Disease Control (CDC) in the USA recommended early screening for children over 10 years who present with any clinical signs of the metabolic syndrome (central obesity, raised blood pressure, raised triglycerides, low HDL-C, and fasting hyperglycemia), family history of T2DM, those who are members of particular ethnic group at risk (Asians, Hispanics, Indians and African Americans), and if they are extremely obese with BMI of $>30 \mathrm{~kg} / \mathrm{m}^{2}$ and fall in the $>95^{\text {th }}$ percentile (Reineher, 2005; Singh, Shaw, \& Zimmet, 2004). Although research is lacking on Jamaican adolescents, it was hypothesized that Jamaican adolescents, the majority of whom, are of African descent (91\%), mixed ethnicities (7\%), Indian (1\%) and others (1\%) (Jamaica Facts and Figures, 2005) will share the same dynamics as observed by the other ethnic groups in other countries.

The proposed study involved screening for overweight and obesity and lifestyle factors, such as physical activity levels and diet that may increase the risk of T2DM and CVDs among 276 randomly selected Jamaican adolescents in ten high schools in Jamaica. In addition, blood samples were tested for fasting blood glucose (FBG), total cholesterol (TC), and glycated hemoglobin (HbAlc) using a finger prick method. This assessment is easy to apply in the school setting and offers fast feedback. The finger prick can be used as a screening tool to identify those students in need of further followup, and is an inexpensive and less invasive data collection tool when compared to venipuncture, a more involved and invasive screening method, more appropriate for a clinical setting. A sub-sample of 59 randomly selected students from the larger study group was tested using venipuncture for the same blood parameters. Testing of this sub- 
sample was used to validate the finger prick method versus venous blood testing to determine if the former can be used for effective screening of risk factors forT2DM and CVDs in a school setting. Other data that were collected included anthropometric measures such as weight, height, body mass index (BMI), waist circumference (WC), waist-to-hip ratio (WHR), demographic data, blood pressure (BP), presence of Acanthosis Nigricans (AN), dietary intakes of energy and macronutrients, physical activity (PA) levels, family history of overweight and obesity, T2DM, and CVDs.

\section{Specific Aims}

The main aim of this study was to examine Jamaican adolescents, ages 14-19 years for risk factors of T2DM and CVDs. Specific aims were to:

1. determine the prevalence of risk factors for T2DM and CVDs among Jamaican adolescents.

2. identify the dietary, family history and physical activity levels which contribute to the risk factors for T2DM and CVDs in Jamaican adolescents.

3. characterize the frequency and cluster of risk factors for T2DM and CVDs, such as diet, family history of T2DM (first degree relative with diabetes), CVDs, obesity $\left(\mathrm{BMI}>95^{\text {th }}\right.$ percentile), hypertension, high-risk ethnic groups (Blacks and Indians), high levels of $\mathrm{HbAlc}$ and FBG, and low physical activity levels among Jamaican adolescents.

4. compare blood samples of $\mathrm{FBG}, \mathrm{TC}$, and $\mathrm{HbA} 1 \mathrm{c}$ using finger prick method with that of venipuncture to ascertain if the former can serve as a surrogate for the gold standard of venipuncture. 
Table 1

Research Questions, Objectives and Hypotheses

\section{Research Questions $\quad$ Objectives Hypotheses}

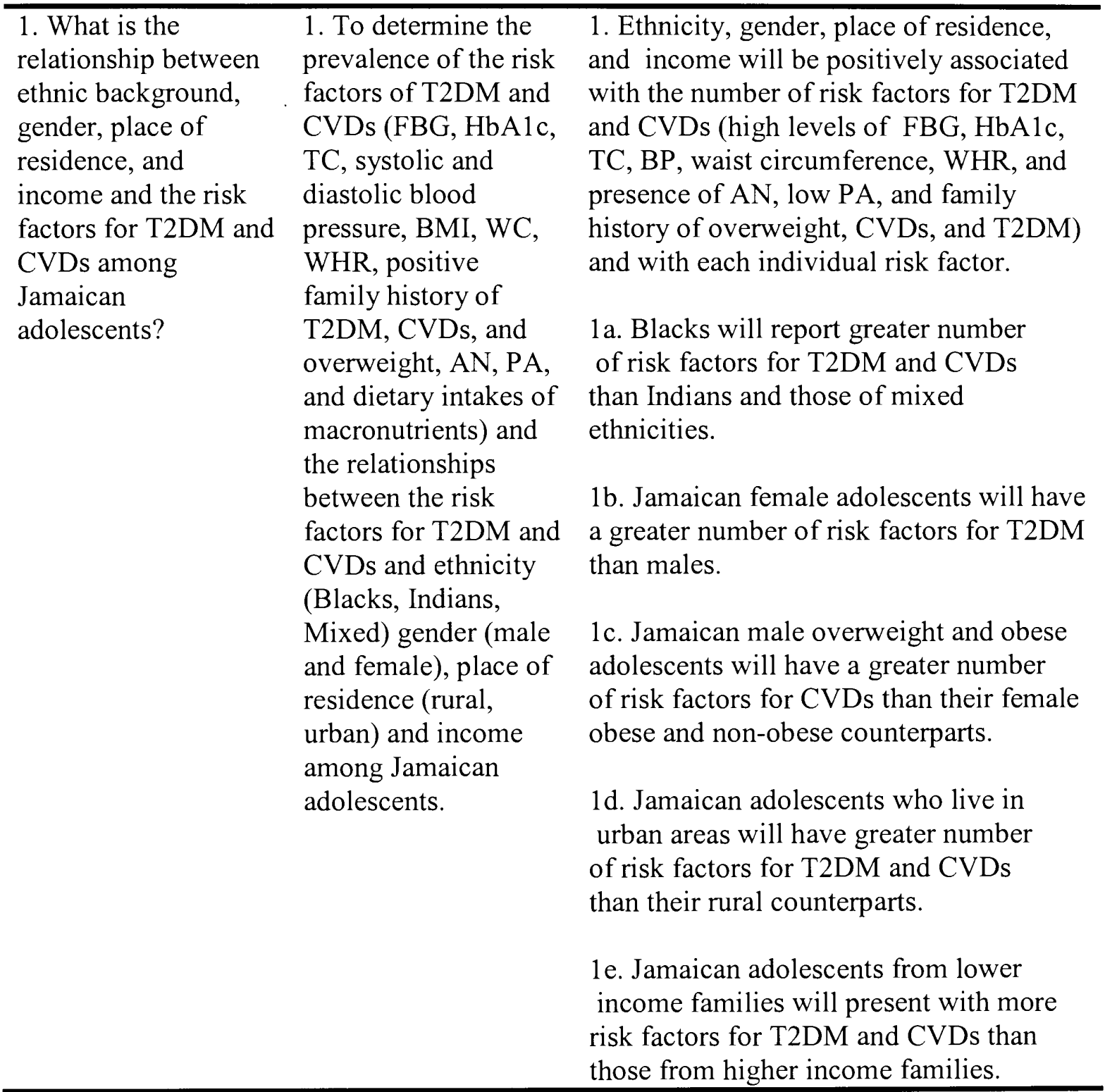


2. What is the

prevalence of risk

factors for developing

T2DM and CVDs

among Jamaican

adolescents?
2. To determine the frequency, cluster, and associations of the risk factors of T2DM and CVDs in Jamaican adolescents using measures such as FBG, HbA1c, TC, systolic and diastolic blood pressure, BMI, WC, WHR, positive family history of T2DM, CVDs, and overweight, AN, PA levels, and dietary intakes of macronutrients.
2. Overweight and obese Jamaican adolescents will have higher levels of at least one of the following risk factors: high FBG, HbAlc, TC, BP, WC, WHR, and presence of AN, low PA, and family history of overweight, CVDs, and T2DM, than those with normal or low BMI.

2a.Waist circumference and WHR of Jamaican adolescents will be positively associated with the risk factors for T2DM and CVDs (high FBG, HbAlc, TC, BP, subjects' $\mathrm{BMI}$, and presence of AN, low PA, and family history of overweight, CVDs, and T2DM), and with each individual risk factor.

2b. Jamaican adolescents with family history of obesity (parents' BMI) will present with $>$ BMI than those without family history of obesity as determined by anthropometric measures of adolescents and self-reported data of parents/family.

2c. Energy intakes of Jamaican adolescents will be positively correlated with the total number of risk factors for T2DM and CVDs (high levels of FBG, HbA1c, TC, BP, parents' BMI, waist circumference, WHR, and presence of AN, low PA, and family history of CVDs, and T2DM) and with each individual risk factor. 
2d. Intakes of complex and refined carbohydrates of Jamaican adolescents will be positively associated with the total number of risk factors for T2DM and CVDs (high levels of FBG, HbA1c, TC, BP, waist circumference, WHR, and presence of AN, low PA, and family history of overweight, CVDs, and T2DM) and with each individual risk factor.

2e. Total fiber intake (g) of Jamaican adolescents will be negatively associated with the total number of risk factors for T2DM and CVDs (high levels of FBG, HbAlc, TC, BP, waist circumference, WHR, and presence of AN, low PA, and family history of overweight, CVDs, and T2DM) and with each individual risk factor.

$2 \mathrm{f}$. Intakes of saturated and unsaturated fat, and intake of omega- 3 and omega- 6 fatty acids of Jamaican adolescents will be associated with the total number of risk factors for T2DM and CVDs (high levels of FBG, HbA1c, TC, BP, waist circumference, WHR, and presence of AN, low PA, and family history of overweight, CVDs, and T2DM) and with each individual risk factor. 


\begin{tabular}{|c|c|c|}
\hline Research Questions & Objectives & Hypotheses \\
\hline $\begin{array}{l}\text { 3. Is there a } \\
\text { relationship between } \\
\text { blood parameters } \\
\text { such as glucose, TC, } \\
\text { and HbAlc measured } \\
\text { by finger prick } \\
\text { method and the same } \\
\text { measures using } \\
\text { venipuncture? }\end{array}$ & $\begin{array}{l}\text { 3.To compare the } \\
\text { results of blood } \\
\text { parameters using finger } \\
\text { prick techniques with } \\
\text { that of venipuncture to } \\
\text { determine if there is a } \\
\text { relationship between } \\
\text { the two methods and } \\
\text { determine if the finger } \\
\text { prick method can be } \\
\text { used instead of } \\
\text { venipuncture in the } \\
\text { early screening of } \\
\text { T2DM and } \\
\text { cardiovascular } \\
\text { diseases. }\end{array}$ & $\begin{array}{l}\text { 3. There will be no significant } \\
\text { differences between, FBG, TC, and } \\
\text { HbAlc measured by finger prick and } \\
\text { venipuncture methods. }\end{array}$ \\
\hline
\end{tabular}

Risk Factors for T2DM and CVDs

Childhood obesity and overweight are strong predictors of T2DM and CVDs, the prevalence of which has sharply increased globally within the past two decades (Rubenstein, 2005; James, Leach, \& Kalamara et al, 2001). Obesity is defined as "an excess of body fat mass exceeding $20 \%$ of total body weight." The World Health Organization (WHO) estimated that over one billion adults in the world are overweight with 300 million being clinically obese that is, BMI over $20 \%$ of ideal weight for height (WHO, 2003). Of significance is the rising prevalence in adolescents since obesity affects morbidity and mortality in young adulthood (Rubenstein, 2005). The growing prevalence of obesity and related chronic diseases that originated in the industrialized countries have now become diseases of the developing countries, many of which are still fighting infectious childhood diseases (Prentice, 2006; Nugent, 2006; Popkin, 2001).

Data on obesity in the developing world are sparse, however Delpeuch \& Maire, (1997) 
estimated that over $30 \%$ of people in Latin America, the Caribbean, the Middle East, and Northern Africa are overweight.

Overweight varies by race and ethnicity, place of residence (rural or urban) and gender (male /female). Data from US based studies revealed that up to $14 \%$ nonHispanic whites, 21\% non-Hispanic blacks, and 23\% Mexican American adolescents are overweight (McKnight-Menci et al, 2005). Place of residence is a predictor of overweight as more people move from rural to urban areas and adapt to urban lifestyles. According to the Food and Agricultural Organization (FAO, 2006) up to $50 \%$ of the world's population now live in urban areas. The change from a rural to urban lifestyle increases the risk for obesity with a corresponding increase in the intakes of high energydense foods, and reduced physical activity (Popkins, 2001; Prentice, 2006). In Jamaica, $52 \%$ of the population lives in urban areas and are mostly centered in four of the fourteen parishes; Kingston, St. Andrew, St. Catherine and St. James (STATIN, 2001). The first three of these parishes were targeted for this research.

Data on gender differences vary with some studies indicating that more Indian boys $(17.8 \%)$ than girls (15.8\%) in India, ages $14-19$ years are overweight (Ramachandran, 2004). Other studies indicated that obesity is more prevalent in women worldwide partly due to their constant exposure to a domestic environment and easy access to food (James et al, 2001). In a study on obesity and overweight in Jamaican children, more girls than boys, ages 10-15 years were found to be obese (Gaskin \& Walker, 2003).

Obesity is linked to T2DM and CVDs. Type 2 diabetes mellitus is a metabolic disease characterized by glucose intolerance and IR often associated with obesity 
(McKnight-Menci, et al., 2005). The disease might go unnoticed for years while the increased hyperglycemia damages vital organs (McKnight-Menci, et al, 2005). Further, T2DM results in "a gradual increase in insulin resistance associated with a decline of insulin secretion with fasting hyperglycemia" (Marcovecchio, Mohn, \& Chiarelli, 2005). T2DM in children is mostly linked to obesity, sedentary lifestyle, puberty, race, ethnicity, features of insulin resistance, family history of T2DM, being female, and perinatal factors (Marcovecchio et al, 2005; Berry, Urban, \& Grey, 2006).

There is a strong association between the incidence of T2DM and overweight/obesity. Type 2 diabetes mellitus accounts for up to $85 \%$ of all cases of diabetes. Of this percentage, approximately $95 \%$ of individuals with T2DM are overweight or obese (WHO, 2003). Once considered an adult-onset disease until the late 1970s, T2DM is now commonly seen in younger populations especially among adolescents and minorities (Fagot-Campagna, 2000; McClain, Srinivassan, \& Chen, et al, 2000 ). Up to $8-45 \%$ of newly diagnosed cases of T2DM in children over 10 years occur among American ethnic minorities (ADA, 2005). Type 2 diabetes mellitus is now accepted to run parallel to the increasing rates of obesity and is spreading to the developing countries (Gungor, \& Arslanian, 2002). It is estimated that by the year 2025, most cases of T2DM will come from India, and the Middle Eastern countries, with large increases expected from China, Latin America and the Caribbean and the rest of Asia (WHO, 2003).

The prevalence of CVDs is increasing in the lesser developed countries. Approximately $80 \%$ of all cases of CVDs now occur in the developing countries (Nugent, 2006). Up to $17 \%$ of deaths in the developing countries were attributed to CVDs in 2000 
(Nugent, 2006). Data on heart diseases in Jamaica indicated that of 15,511 ECGs performed in 2004, $23.1 \%$ had abnormalities; out of 44,127 persons who had their blood pressure checked, $68.3 \%$ had readings above the normal range (Heart Foundation of Jamaica, 2006). These screenings were carried out by the Supermarket Screening Program (SSP) of the Heart Foundation of Jamaica and involved only screening of adults. Increased rates of obesity are seen even in countries with low GDP such as Pakistan and Nigeria that now face significant risks from obesity and high cholesterol leading to cardiovascular diseases (Nugent, 2006). Although Jamaica has a per capita GDP of US\$3,350.00 (Jamaica Facts and Figures, 2005), the country may still experience a high prevalence of obesity and overweight. Despite the low per capita income of Jamaicans, there is evidence of overweight among the adult population. Of the 15,511 adults who had their weight checked in the SSP, $35.3 \%$ were overweight, $23.4 \%$ obese, and $2 \%$ were morbidly obese. According to Nugent (2006) "the health-related problems of overweight and obesity now widen the health disparities between rich and poor and add extra burden on the health care systems of the developing countries which are considered the most vulnerable victims of non-communicable disease related to obesity." Obesity poses economic strain on the already stressed economy of a nation.

The present study determined whether Jamaican adolescents were at risk for T2DM and CVDs and examined the relationship between obesity and type 2 diabetes mellitus and risks of cardiovascular diseases in this age group. The International Obesity Task Force (IOTF) suggested using the BMI for weight and height $\left(\mathrm{kg} / \mathrm{m}^{2}\right)$ as a reasonable measure of obesity in children and adolescence and was used as a benchmark for determining overweight and obesity among the Jamaican population studied. 


\section{T2DM and Obesity among Adolescents}

The WHO estimated that globally, at least 171 million people suffer from T2DM and predicted that by the year 2030 this number will double (WHO, 2006). This increase is expected from the present population therefore research is needed to help prevent the spread of T2DM. Type 2 diabetes mellitus is most common in developed countries where its prevalence is concomitant to the rising rates of obesity. However, the trend is fast growing in the developing world due to urbanization and lifestyle changes, and most importantly the adaptation of a Western diet and lifestyles (Prentice, 2006; Monteiro Conde, Liu, et al, 2004; Popkin, 2001; Martorell, Khan, \& Hughes et al, 1998). Research shows that diabetes in the English-Speaking Caribbean is on the rise and is projected to continue rising (Hennis, \& Fraser, 2004). According to Henis \& Fraser (2004) the increasing rate of diabetes in the Caribbean mirrors that of Western acculturation as seen in Africa and is associated with the rise in obesity.

Most of the research on type 2 diabetes in children and adolescents has been done in the western world. However, reports from countries such as Japan, Taiwan and India indicated that type 2 diabetes in children and adolescents is on the rise (Singh, et al., 2004). Howdle \& Wilkin (2001) stated that "the number of adolescents with T2DM has increased ten-fold over the past ten years." The rapid rise of T2DM among children was first reported in the Pima Indian population in Arizona where diabetes in adolescents increased by $54 \%$ between 1980s and 1990s (Dabelea, Hanson, \& Bennett et al., 1998; Nesmith, 2001). Despite the scarcity of research on T2DM in children of the developing world, there have been reports of childhood diabetes in countries such as Saudi Arabia, Libya, India, Bangladesh, Mexico, Thailand, and Argentina. In the past, 
most of these cases related to type1 diabetes, but countries such as Japan and Taiwan are now reporting increasing rates of T2DM in children (Singh et al., 2004). This chronic disease is expected to rise with a 5-6\% increase between 2003-2005. The greatest increase is expected from the developing countries, especially India (Singh et al, 2004).

\section{Obesity and T2DM in Jamaican Adolescents}

Research on diabetes in Jamaica does not focus on the younger age groups despite the fact that some studies (Daniels, 2006; Choudbury, 2005, Reinehr, 2005) have shown increasing prevalence of obesity and related T2DM among younger age groups in other countries. Currently Jamaica's population consists of approximately $1 \%$ Indian origin and $91 \%$ of African ancestry with approximately $28 \%$ of the population being between the ages of 10-24 years, most of whom live in the rural areas (National Center for Youth Development, Ministry of Education, Youth and Culture, Jamaica, 2002). Research indicated that;

1. pediatric T2DM is on the rise worldwide (Daniels, 2006; Sacks et al., 2002; Choudbury, 2005).

2. most of the new cases will be comprised of those of Indian origin (Ramchandaran, 2004), one of the Jamaican minorities.

3. African ancestry is a major risk factor for T2DM in studies conducted in the USA, (ADA, 2005; Berry et al., 2004), and those of African ancestry comprise the majority in Jamaica.

This research examined the risk factors for diabetes among adolescents of these two ethnic groups in Jamaica. 
In a 1995 survey of 2,109 Jamaicans 15 years and older, it was reported that "Jamaica has a point prevalence of diabetes of $17.9 \%$ according to WHO criteria and $1.7 \%$ had impaired glucose tolerance (IGT)" (Ragoobirsingh, Lewis-Fuller \& Morrison, 1995). Of this sample, $48 \%$ of the total diabetes prevalence comprised newly diagnosed cases. Diagnosis of diabetes or IGT was based on WHO criteria (fasting blood glucose (FBG) of $\geq 6.7 \mathrm{mmol} / 1$ or a 2 hour postprandial reading of $11.1 \mathrm{mmol} / \mathrm{l}$ ) which represents diabetes, and $\mathrm{FBG}<6.7 \mathrm{mmol} / \mathrm{l}$ and a 2 hour postprandial reading of between $6.7-11.1$ mmol/l indicated the presence of IGT) (Ragoobirsingh, et al., 1995). In this study, 24.6\% of the total participants and $2.6 \%$ of those diagnosed with diabetes fell in the age group 15-24 years which provided limited but enlightening information on diabetes in adolescents. No data on ethnic differences were reported. Gender differences although not significantly correlated with diabetes, showed more females $(54.3 \%)$ than males $(45.7 \%)$ had diabetes. Other factors that correlated positively with the prevalence of diabetes in this sample included advanced age (20.3\% of sample 75 years and over had diabetes), lower level of education, truncal (abdominal) obesity, family history of diabetes, history of alcohol consumption, and multiple parity. Gender, place of residence, birth weight, and cigarette smoking were negatively correlated with diabetes (Ragoobirsingh et al, 1995). In this survey no distinction was made between type 1 and type 2 diabetes mellitus, or was there any mention of the relationship between obesity and other risk factors of diabetes. Some of these findings were corroborated by Mckeigue, Shah, \& Marmot (1991) in Afro-Caribbean men living in Britain.

The current study was the first of its kind to screen Jamaican adolescents at risk of obesity, T2DM, and other nutrition and lifestyle-related health complications. The study 
also determined whether fasting finger prick testing of capillary whole blood for fasting blood glucose (FBG), total cholesterol (TC), and glycated hemoglobin (HbAlc) could be used as a surrogate for the gold standard of venipuncture for early screening of risk factors for diabetes and cardiovascular disease in a school setting. The study adds to the body of literature on T2DM in adolescents in the developing countries. The information furnished by this study will help to forecast the future burden of life-style diseases in Jamaica and for planning the allocation of scarce public health resources.

Relationship of Obesity to T2DM and CVDs

Excess body fat, abdominal and subcutaneous fat are associated with insulin resistance (IR), a key feature of T2DM (Vickram, Tandon, Misra et al, 2005). Obesity strongly predicts T2DM in children and in one study was shown to occur mainly in Indian, Pakistani and Middle Eastern children in the United Kingdom (Vickram et al, 2005). Further, body weight is strongly associated with insulin resistance. Higher BMI is associated with hyperinsulinemia and insulin resistance (Ford, Williamson, \& Liu, 1997). Insulin resistance refers to "reduced responsiveness of peripheral tissues to insulin due to a reduction in the number and function of insulin receptors" (Ford et al., 1997). Further, hyperinsulinemia results when the body's resistance to insulin causes more insulin to be produced by the pancreas (Steinberger \& Daniels, 2003). Obesity is also associated with increased insulin production by pancreatic beta cells. The relationship between obesity and insulin resistance "may be due to the duration and extent of the obesity leading to further deterioration in glucose homeostasis when $\beta$ cells 
become impaired, resulting in reduced insulin production leading to diabetes" (Ford et al, 1997).

It has been suggested that "obesity accompanied by elevated free fatty acids may increase hepatic glucose production and impact insulin resistance of target cells leading to non-insulin dependent diabetes mellitus (NIDDM) or T2DM" (Reaven, 1988). Further support on obesity and insulin resistance comes from Ramachandran (2004) who stated that "insulin resistance is one of the major etiological factors for diabetes, and the risk association of obesity with diabetes is greatly mediated through insulin resistance." Other research showed serum insulin levels to be positively correlated with BMI, LDL-C, glucose, triglycerides, systolic and diastolic blood pressure according to the Bogalusa Heart Study. Negative correlations were found for insulin levels and HDL-C in this study (Bao, Srinivasan, \& Berenson, 1996).

The risk conferred by obesity to the development of diabetes is more than 40 times higher in obese women in comparison to those who are not obese (Ramachandran, 2004). In a 2002 World Health Report, up to $5 \%$ of diabetes, and $21 \%$ of ischemic heart disease cases were attributable to BMI above $21 \mathrm{~kg} / \mathrm{m}^{2}$ (WHO, 2003). The relationship between obesity and hypertension, high cholesterol and diabetes is so strong that researchers now advocate that a diagnosis of obesity can serve as a surrogate for these conditions without the need for blood testing (Nugent, 2006).

Although mortality from cardiovascular diseases has decreased since the 1970s, it is still the main cause of death in the Western World (Health Survey for England, 1999). Data from the Health Survey for England on the Health of Ethnic Minority Groups revealed that some ethnic minority groups appear to be more prone to ischemic heart 
diseases than others. Immigrant men born in the Indian sub-continent had higher mortality than those from the Caribbean. Cerebrovascular disease ranks next to ischemic heart disease as a major cause of death for these same two groups with Black Caribbean men twice as likely to die from strokes, than native men in England and Wales ("Health Survey for England," 1999). In this survey, subjects were classified as having CVD if they had any of these conditions- angina, heart attack, stroke, heart murmur, irregular heart rhythm, high blood pressure or diabetes as confirmed by a nurse or doctor. Despite having lower prevalence of angina, both Black Caribbean men (7.8\%) and women (7.9\%) had higher prevalence of diabetes than the other minority ethnic groups (Indian Men = $7.7 \%$; Irish men $=4.2 \% ;$ Indian women $=4.7 \%$; Irish women $=2.4 \%$ ).

In this survey, individuals of both sexes and all minority ethnic groups classified with CVDs also had BMI> $25 \mathrm{~kg} / \mathrm{m}^{2}$ ("Health Survey for England," 1999). The observed prevalence for overweight and obesity was higher for Indian and Bangladeshi women when adjusted for age. The prevalence of raised total cholesterol $(>6.5 \mathrm{mmol} / \mathrm{l})$ was higher for persons with CVDs than those without. The risk ratio for CVDs was higher for Indian and Pakistani women and Chinese men. The risk ratios for prevalence of angina for Black Caribbean men and women were 1.9 and 2.2 respectively. High blood pressure ( $\geq 140 / 90 \mathrm{mmHg}$ ) was also higher among individuals with CVDs. 


\section{CHAPTER II \\ REVIEW OF LITERATURE}

Recent research shows an increasing global prevalence of T2DM in children and adolescents due to the rising prevalence of obesity (Reinehr, 2005). Overweight is associated with T2DM, CVDs, hypertension, dyslipidemia and insulin resistance. These health conditions of obesity were once thought to relate only to adults but are now increasingly found in children and adolescents. Many of these conditions such as heart attack and strokes are chronic and present physiological changes during childhood. They take decades to present as overt diseases and threaten to shorten the life span by as much as 5 years (Daniels, 2006; Wyatt et al., 2006). Being obese during childhood, adolescence and young adulthood may accelerate these conditions (Daniels, 2006; Choudbury, 2005).

Detection of the early signs of T2DM and CVDs is needed to help in the prevention of these and other related medical conditions. Other studies indicated that about 60 percent of overweight US children also have hypertension, hyperinsulinemia, and/or hyperlipidemia (Freedman et al., 1999). Research is needed on the Jamaican adolescents since it is expected they will share similar characteristics to adolescents in the USA and other countries. Data from this study will help to guide interventions to reduce the risk of morbidity and mortality among the Jamaican population, and the tremendous cost of these life-style diseases for a resource-limited country. 
Type 2 Diabetes Mellitus (T2DM)

Type 2 diabetes mellitus (T2DM), is often called adult-onset or obesity related diabetes although it is now commonly seen in children and adolescents. Type 2 diabetes mellitus is characterized by the body tissues being resistant to insulin. It is the most common form of diabetes and is highly prevalent in adults in the developing world. The majority of cases of T2DM are seen in obese persons or those who reported history of obesity at the time of diagnosis. Type 2 diabetes mellitus can occur at any age but is usually diagnosed after age 30 years. Causes of T2DM include defects in both the secretion and action of insulin. With T2DM, there are normal levels of endogenous insulin but levels are inadequate to overcome concomitant insulin resistance. Insulin resistance (IR) occurs when there is a decrease in tissue sensitivity or responsiveness to insulin leading to hyperglycemia (Mahan \& Escott-Stump, 1996).

Three possible causes of T2DM have been suggested; 1) an abnormal pattern of insulin secretion that can be inadequate or excessive; 2) decreased uptake of insulin at the cellular level resulting in increased elevation in postprandial glucose levels related to a defect in a receptor or post-receptor in the cells; and 3) poor release of glucose from the liver early in the morning which results in elevation in fasting glucose levels. These three defects then lead to a continued state of hyperglycemia which further compounds the problems of insulin resistance and secretion (Mahan, \& Escott-Stump, 1996).

Although impaired glucose tolerance (IGT) is not a diagnosis for diabetes, people with IGT eventually develop diabetes mellitus. IGT is manifested by higher than normal blood glucose. Early screening for IGT can help to prevent T2DM (Mahan, \& EscottStump, 1996). Most cases of T2DM can be treated with medications but lifestyle 
changes can help to manage the disease. Screening is therefore necessary so that early detection can help to prevent complications such as cardiovascular diseases, chronic renal failure, retinal damage and possibly blindness, nerve damage, erectile dysfunction, gangrene, and risk of amputations. Other factors such as smoking, hypertension, elevated cholesterol, obesity, and lack of regular exercise can worsen the effects of diabetes (Mahan, \& Escott-Stump, 1996).

\section{Recognizing T2DM in Children}

Detection of T2DM in children is even more problematic than in adults. Type 2 diabetes mellitus in children is sometimes misdiagnosed as there are cases of children with T2DM who present with diabetic ketoacidiosis (DKA), a condition associated with type I diabetes. Studies indicated that up to $42 \%$ of African American children and $30 \%$ Hispanic children with type 2 diabetes also presented with DKA (Pinhas-Hamiel, Dolan \& Zetler, 1997). However, diabetes in children and adolescents can be easily recognized by the presence of Acanthosis Nigricans (AN) in the absence of DKA (Scott, Smith, Cradock et al., 1997). Acanthosis Nigricans is reported to occur in $60-90 \%$ of young people with type 2 diabetes (Scott, et al., 1997). The presence of AN (manifested by a dark ring around the neck) is a sign of insulin resistance (IR) and is commonly seen in Native Americans (40\%), African Americans (13\%) and Hispanics (6\%) (Tajima, 2002; Stuart, Driscoll, \& Lindquist et al., 1998). The presence of AN alone is not conclusive for determining the presence of insulin resistance or hyperinsulinemia, neither does the presence of AN in minority groups. In a study by Nguyen, Keil, \& Russell et al. (2001), approximately one-third of the obese children with hyperinsulinemia also had AN among 
all ethnicities. One study shows that BMI is a better marker than the presence of AN for detecting IR (Hirschler, Aranda, \& Oneto, et al, 2002). The presence of AN is likely to correlate with BMI (Hirschler et al., 2002). Therefore, we used different indices for determining T2DM in our population such as measures of BMI, FBG, HbAlc and the presence of AN.

According to the American Diabetes Association (ADA) 2005, 8-45\% of minority children above 10 years who have diabetes are diagnosed with type 2 diabetes (Berry et al., 2006). With the increase in numbers of children diagnosed with type 2 diabetes, it is imperative that primary prevention aims at delaying its development by reducing overweight among children (Berry et al., 2006). Those children who are already diagnosed with pre-diabetes should be monitored to prevent further development to diabetes. It is also important to control the disease to prevent further complications such as hypertension, hyperlipidemia, and cardiovascular diseases (Rosenbloom \& Silverstein, 2003).

Obesity and Cardiovascular Diseases

Obesity has been cited as "a risk factor for coronary heart disease, ventricular dysfunction, congestive heart failure, stroke, and cardiac arrhythmias" (Klein, Burke, Bray et al, 2004). Obesity increases mortality from CVDs and deaths from CVDs are directly related to increase in BMI. Persons with BMI $>35 \mathrm{~kg} / \mathrm{m}^{2}$ are $2-3$ times more likely than lean persons with lower BMI (18.5- $\left.24.9 \mathrm{~kg} / \mathrm{m}^{2}\right)$ to die from CVDs (Calderon, Boyne, \& Bennett, et al, 2004). The authors further stated that "overweight in adolescent 
is associated with a $130 \%$ increased risk of CVDs mortality during adulthood" (Calderon et al., 2004).

Although CVDs do not manifest until adulthood, studies have shown that the atherosclerotic process begins early in life (Berenson, Wattigney, Bao et al., 1995; Berenson, Srinivasan, Bao et al., 1998; Chen, Bao, Begum, et al., 2000). Overweight in children has been linked to "increased rates of hypertension, hyperlipidemia, T2DM, and early atherosclerotic lesions" (Calderon, Yucha, \& Schaffer, 2005). Increase in body weight results in increased blood pressure (Calderon et al, 2004). More compelling evidence on overweight and risk of CVDs comes from Hoffman, Krombout, \& de Lezene (1988) who found strong associations between morbidity and mortality from adults who were overweight as adolescents.

Obesity and hypertension are strongly linked to adult age, however, there is growing evidence that hypertension also occurs among younger age groups (Sorof, Poffenbarger, \& Bernard, et al., 2002). The majority of children over six years of age who have hypertension also have a family history of hypertension, and are usually overweight. Along with hypertension are other medical conditions such as kidney diseases and disorders of the endocrine system or abnormalities of the blood vessels. Other factors are inadequate physical activity, poor diet, and poor stress management. Hypertension is a major risk factor for stroke and heart attacks. Overtime, high blood pressure can damage vital body organs such as the kidney, brain and heart, therefore early detection is necessary.

To classify as hypertensive, an individual must have persistently elevated systolic and/diastolic arterial BP of 140/90 mmHg or more if they are over 15 years old. BP 
measurements are usually spontaneous and vary greatly. It is suggested that $\mathrm{BP}$ be taken after 10 minutes of quiet rest. If the first reading of BP is greater than $120 / 80 \mathrm{~mm} \mathrm{Hg}$ or if the difference between two readings is $>$ than $5 \mathrm{~mm} \mathrm{Hg}$ further readings should be taken. Hypertension is diagnosed based on more than one reading. Readings must be taken on different occasions and separated by one to two weeks.

Several epidemiological studies have found that BP was significantly higher for children with higher BMI. Obese adolescents were more likely to have three times greater prevalence of hypertension than their non-obese counterparts (Sorof et al., 2002). It is difficult to determine a cut-off point for hypertension among children since BP continues to rise from infancy to about age 18. Therefore, BP for children and adolescents is determined by plotting each individual's height on standard age-adjusted growth curves to determine a height percentile. The height percentile, age and gender are then used to ascertain the $90^{\text {th }}$ and $95^{\text {th }}$ percentiles for BP. These tables of percentiles are available from the 1996 National High Blood Pressure Education Program Working Group on Hypertension Control in Children and Adolescents. Children who plot below the $90^{\text {th }}$ percentile for height, age and gender are considered to have normal BP. Children who plot between $90-95^{\text {th }}$ percentile are classified as having "pre-hypertension" which is likely to develop into hypertension. Children whose systolic or diastolic BP plots higher than the $95^{\text {th }}$ percentile for age, gender, and height measurements, are classified as hypertensive. Three measurements of systolic and diastolic BP higher than the $95^{\text {th }}$ percentile are needed to classify one as hypertensive since BP varies with emotions, activity level, medications, and stress (Collins, Peto, \& McMahon, 1990). Adolescents with $\mathrm{BP}>120 / 80 \mathrm{mmHg}$ are considered to be pre-hypertensive. For 17 year olds, the 
$95^{\text {th }}$ percentile is close to $140 / 90 \mathrm{~mm} \mathrm{Hg}$ which is the cut-off point for adults. Use of the height percentiles for determining hypertension can be misleading for younger age groups. Organizations such as the American Academy of Pediatrics (AAP), American Heart Association (AHA), and the American Medical Association (AMA) "recommend routine screening of adolescents and children during preventive visits since up to $28 \%$ of children have secondary hypertension for which early detection may be beneficial."

\section{Determinants of Cardiovascular Risks}

Research indicated that atherosclerosis began in childhood McGillis-Bindler, \& Bruya, 2000; Steinberger et al., 2003; Gungor, \& Arslanian, 2005). Therefore, testing for cholesterol in children can help to prevent later development of CVDs. It was recommended that "children should be tested for cholesterol levels if a parent or grandparent had atherosclerosis at or before age 55, the parent or grandparent suffered a heart attack, or showed other signs of artery disease at or before age 55, or if a parent has a blood cholesterol level over $240 \mathrm{mg} / \mathrm{dL}$ " (NCEP, 1995). Subjects with T2DM tend to have low HDL-C and high levels of triglycerides. The American Dietetic Association recommended lowering LDL and total cholesterol as well as monitoring blood pressure for persons with diabetes. Screening for high cholesterol, low HDL-C and high triglycerides is needed in the early prevention of T2DM.

Cholesterol guidelines for children were set by the National Cholesterol Education Project sponsored by the National Heart, Lung and Blood Institute. A desirable blood lipid profile for an adult is TC $<200 \mathrm{mg} / \mathrm{dl}$ and HDL-C $\geq 35 \mathrm{mg} / \mathrm{dl}$. For children, cholesterol level less than $175 \mathrm{mg} / \mathrm{dL}$ was recommended. LDL-C is not easily 
measured by laboratory methods but can be calculated. LDL-C and TC are highly correlated. Therefore, TC levels are used to detect LDL-C. Measurement of a complete lipid profile includes TC, LDL-C, HDL-C and triglycerides (TG). Measures of LDL-C can be determined by measuring TC, TG and HDL-C. Total cholesterol can be measured in a non-fasting state. However, TG must be measured after a 12 hour fast to allow time for the chylomicrons to clear. LDL-C is calculated using the Friedwald formula:

$$
\text { LDL-C }=(T C)-(H D L-C)-(T G / 5)
$$

Where LDL-C = low-density lipoproteins cholesterol; TC = total cholesterol; $\mathrm{HDL}-\mathrm{C}=$ high density lipoprotein cholesterol; and $\mathrm{TG}=$ triglycerides. Use of this calculation is best done when TG levels are less than $400 \mathrm{mg} / \mathrm{dl}$. A desirable lipoprotein profile for children would then be TC $\leq 170 \mathrm{mg} / \mathrm{dl}$; LDL-C $\leq 100 \mathrm{mg} / \mathrm{dl}$; and HDL-C $\geq 60$ mg/dl (NCEP, 1993). A child's LDL-C should be checked if the child has a TC of 170 $\mathrm{mg} / \mathrm{dL}$ or greater. Triglycerides are also measured when the LDL-C is checked. TG levels less than $200 \mathrm{mg} / \mathrm{dL}$ are considered normal (Mahan, \& Escott-Stump, 1996).

\section{Definition of Overweight and Obesity}

An adult is considered overweight if the BMI is $25.0 \mathrm{~kg} / \mathrm{m}^{2}$ or more, and obese if the BMI is $\geq 30 \mathrm{~kg} / \mathrm{m}^{2}$. BMI is defined as the weight in kilograms divided by the square of the height in meters $\left(\mathrm{kg} / \mathrm{m}^{2}\right)$. BMI is generally considered a crude estimate of body fat. BMI as an assessment of measuring obesity in adults has been internationally accepted and correlated well with body fat $r=.7-.8$ (Epstein \& Higgins, 1992). Different indices have been used to determine obesity in children and include the recommendations of the International Obesity Task Force (IOTF) which suggests using the BMI for weight 
and height $\left(\mathrm{kg} / \mathrm{m}^{2}\right)$ as a reasonable measure of obesity in children and adolescents. The IOTF further stated that the standards used for grading obesity such as grade I and grade II for BMI of 25 and 30 respectively should be used (Dietz \& Bellizzi, 1999). Other measures include the NHANES which uses $>$ the $85^{\text {th }}$ percentile as a cut-off point for obesity. Countries in Asia and Latin America tend to use a cut-off point of $>120 \%$ of weight for height (Dietz \& Bellizzi, 1999). In 1995 the WHO accepted that the new normal adult range for BMI should be changed from 20.0 to $18.5-24.9 \mathrm{~kg} / \mathrm{m}^{2}$ (James et al., 2001).

Differences in prevalence of obesity vary among countries based on the index used to measure obesity. For example, in Argentina when the NHANES $85^{\text {th }}$ percentile was used, the prevalence of obesity appeared to more than double when compared to the $>120 \%$ of weight for height (Guillaume, 1999). A comparison of the $85^{\text {th }}$ percentile cutoff point for BMI for three countries; Australia, United States and the United Kingdom, revealed an overlap between the BMI for children ages 7-15 years between Australia and the United States while the United Kingdom data were slightly lower. The BMI seem to be more widely accepted for measuring obesity in children but the cut-off percentile varies from $85^{\text {th }}-97^{\text {th }}$ percentile. The use of skin-fold thickness and weight for height are also used in some countries (Guillaume, 1999). The use of central adiposity is not considered suitable since morbidity and mortality in obese children and adolescence have not been well documented (Guillaume, 1999).

Adult BMI varies among ethnic and national groups. The same might be true of adolescents in different countries and of different ethnicities. A WHO Report (2003) found adult mean BMI levels of $22-23 \mathrm{~kg} / \mathrm{m}^{2}$ in Africa and Asia when compared to levels 
of $25-27 \mathrm{~kg} / \mathrm{m}^{2}$ which is common in North America, Europe, some Latin American countries, North Africa, and Pacific Island countries (WHO, 2003). Globally there appears to be an upward shift in BMI levels with corresponding greater risk for high blood pressure, diabetes, and heart disease at an earlier age. Over-nutrition and increased $\mathrm{BMI}$ is accompanied by underweight and decreased BMI in some countries. A BMI of $<$ $18.5 \mathrm{~kg} / \mathrm{m}^{2}$ is considered underweight and can have debilitating health effects. See Table 2 for The WHO standard classification of obesity and risk of co-morbidities

\section{Table 2}

\section{WHO Classification of Obesity and Risk of Co-Morbidities}

\begin{tabular}{lll}
\hline & \multicolumn{1}{c}{$\begin{array}{c}\mathrm{BMI} \\
\mathrm{Kg} / \mathrm{m}^{2}\end{array}$} & $\begin{array}{l}\text { Risk of Co- } \\
\text { morbidities }\end{array}$ \\
\hline Underweight & $<18.5$ & Low \\
Normal BMI & $18.5-24.9$ & Average \\
Overweight & & \\
Pre-obese & $25.0-29.9$ & Increased \\
Obesity class I & $30.0-34.9$ & $\begin{array}{l}\text { Moderate } \\
\text { Obesity class II }\end{array}$ \\
Obesity class III & $35.0-39.9$ & Vevere \\
& $\geq 40$ & Very severe \\
\hline
\end{tabular}

There is concern among Asian investigators over the higher BMI cut-off point for obesity since research has shown a high prevalence of diabetes and hypertension at lower BMI for this group (James et al., 2001). As a result, they have suggested using a cut-off point for obesity of BMI $25 \mathrm{~kg} / \mathrm{m}^{2}$ and an upper limit for normal of $22.9 \mathrm{~kg} / \mathrm{m}^{2}$. Support for this lower BMI limit comes from Ramachandran (2004) who argued that the association between BMI and glucose intolerance is as high as in any other population 
despite having lower BMI. For example, studies have shown that for urban Indian population a BMI of $>23 \mathrm{~kg} / \mathrm{m}^{2}$ increased the risk for diabetes for both males and females (Snehalatha, Viswanathan, \& Ramachandran, 2003). In other words, a BMI of $<23 \mathrm{~kg} / \mathrm{m}^{2}$ is considered healthy for Asian Indians. Consequently, the WHO has established a BMI of $18.5-22.0 \mathrm{~kg} / \mathrm{m}^{2}$ to be healthy for this population. Healthy BMI among other ethnic groups is 18.5-24.9. This ethnic difference in nationality-related BMI is significant to this study, since Jamaica's population comprises up to $1 \%$ Indians, and data from Trinidad revealed a higher mortality rate of diabetes among Indo-Trinidadians when compared to other populations. It is likely that a cut-off point of 24.9 for a healthy BMI among Jamaican Indians may result in misclassification of risk of overweight among this group.

One study of 306 Jamaican children assessed obesity using the IOTF BMI cut-off point for obesity and measures of skin-fold and examined children at age 7-8 and at follow up later at age 11-12. It was found that BMI correlated better with other indices for adiposity among the older age group. Establishing a suitable BMI for children has been a concern of many researchers due to differences in pubertal age. For this population, pubertal age was set at 11-12 years (Gaskin \& Walker, 2003). Results indicated an increase in overweight from 3.5\% - 9.5\% over the follow-up period. The BMI had low sensitivity to identify children $>85^{\text {th }}$ percentile when the IOTF cut-off point for BMI was used.

In the Jamaican study, Gaskin \& Walker (2003) showed that other measures of adiposity such as TSF and SF had higher sensitivity, however, $14-30 \%$ of the children were misclassified. The specificity for BMI was high. In general, girls were fatter than 
boys. It was concluded that children identified for overweight using the IOTF BMI cutoff point are likely to have high body fatness. In another study, testing the adequacy of the BMI cut-off point for children found that use of BMI $95^{\text {th }}$ percentile as cut-off point for children had high sensitivity (88\%) and high specificity $(94 \%)$. However, when lower percentiles were compared using the ROC (receiver operator characteristic) analyses, results indicated that lower cut-offs improved sensitivity with no loss in specificity (Reilly, Dorosty, \& Emmett, 2000).

Dietz \& Bellizzi, (1999) suggested that “a BMI above the $95^{\text {th }}$ percentile may be a more specific measure to identify children and adolescents with increased body fat." What was needed was a mid-point sensitivity and specificity study using the $85^{\text {th }}$ and $95^{\text {th }}$ percentiles. An IOTF conference proposed "a BMI above the $85^{\text {th }}$ percentile as a cut-off for overweight and above the $95^{\text {th }}$ percentile as an index for determining obese adolescents" (Dietz \& Bellizzi, 1999). These results are significant to this study which aims at identifying overweight and obesity in Jamaican adolescents and examining them for risks of T2DM and CVDs.

Prevalence of Overweight and Obesity

For US children, the rates of overweight and obesity increased from 4\% in 1982 to $13 \%$ in 1999 (Headley et al., 2004), and reached 16\% in 2001 (Kumanyika, Jeffrey, Morabia, et al., 2002). Approximately $25 \%$ white children and 33\% African American and Hispanic children were overweight. Roughly $59 \%$ US adults have BMI $>30$ and consequently are at risk to chronic diseases. Research indicated that " $26 \%$ of obese people have hypertension, $80 \%$ present with type 2 diabetes, $70 \%$ have cardiovascular 
diseases related to obesity, $42 \%$ breast and colon cancer diagnosed among obese persons, and 30\% gall bladder surgery are related to obesity" (Kumanyika, et al., 2002). It is estimated that globally over 22 million children are overweight (Kumanyika, et al., 2002). Data from the developing world indicated that approximately $18 \%$ of Indian children ages 13-18 years were overweight (Ramchandaran et al, 2003) due to decreasing physical activity, and higher socio-economic status (SES). Thailand showed an increase of overweight from $12.2 \%$ to $15.6 \%$ over a two-year period among the $6-12$ year olds (Kumanyika et al., 2002). In Latin America, studies were based on younger children 1-5 years but the prevalence of obesity has increased by $56 \%$ between 1985 and 1995 (Kumanyika et al., 2002).

\section{Causes of Obesity}

Obesity has a multiplicity of causes. Fundamentally, weight gain results when the energy intakes exceed energy expenditure. According to the US Surgeon General, up to 300,000 deaths per year occur from sedentary activities and unhealthy dietary habits. Diet, inactivity and increased television viewing have been attributed to the rising prevalence of overweight and obesity among children (Nelson, Carpenter, \& Chiasson, 2004). Other factors that influence obesity are family history of obesity, type of work, level of physical activity, race and environment. Recent evidence points to the influence of the fast food industry and their competitive marketing strategies to super-size their meals (Schlosser, 2001). Portion sizes in a typical fast food restaurant for one of their combination meals may be able to provide all the calories needed for one day. 
The increasing rates of overweight and obesity have also been attributed to changes in diet and consumption patterns. In the US, children are exposed to a wide variety of processed and refined carbohydrate foods. In addition to increased consumption of sodas over milk, children in industrialized countries have access to more meats and high fat foods than children in the developing world. The fast food industry tends to market to the tastes of children who put the pressure on parents to purchase these foods over home-made foods (Schlosser, 2001).

Changes in lifestyle patterns with more mothers working outside the homes leave families with less time to prepare foods and consequently drive the success of the fast food industry. The response is to increase the numbers of fast food restaurants and the portion sizes to outsell competitors. The increase in portion sizes or "super-sizing" is concomitant with the increase rates of obesity among the US population (Schlosser, 2001). The fast food industry has expanded its business to many developing countries with the same marketing strategies aimed at children, and where local families equate the ability to purchase fast foods with affluence. Schlosser (2001) alluded to the growing rate of obesity in Asian countries to the expansion of the fast food industry in those countries. Reports from national data sets in the US indicated increase in consumption of sodas, pasta, salty snacks, juice and sugar-and sweetened beverages among the US population (Smickilas-Wright, Nielson, \& Popkin, 2003)

Along with the increase in portion sizes, physical activity has been declining among children. There have been reports on a link between the amount of time children spent watching television and/ or videos and obesity (Crespo Smith, \& Troiano et al., 2002; Certain \& Khan, 2002; Dennison, Erb, \& Jenkins, 2002; Christakis; Ebel, \& Rivera 
et al, 2004). Research on physical activity and just how much is adequate is sparse, but there is some evidence that children are not sufficiently active physically (Crespo et al, 2002; Dennison et al., 2002; Dowda, Ainsworth, \& Addy et al., 2001; Gortmaker, Must, \& Sobol et al., 1996). The US Surgeon General has recommended that "all individuals over two years old engage in moderate physical activity for at least 30 minutes per day" (Welk \& Wood, 2000).

\section{Ethnicity, Overweight and Obesity}

With regards to ethnicity and overweight, Black girls 5-17 years are generally heavier $(3 \mathrm{~kg})$ and taller $(2-3 \mathrm{~cm})$ than their White counterparts (Dwyer et al., 2000; Krosnick, 2000). When adjusted for height, Black girls remained relatively heavier than White girls. The National Growth and Health Study (NGHS) of girls 9-10 years old living in Virginia, California, Ohio, and Washington, DC (Kimm Barton, \& Obarzanek et al., 2001) showed that Black girls had significantly higher BMI, skinfold measures, and blood pressure compared to their White counterparts. Dwyer et al (2000) examined data from the Child and Adolescent Trial for Cardiovascular Health (CATCH) survey, which was designed to measure the effectiveness of home versus school education programs to reduce cardiovascular risks among elementary school children. The study found that more Blacks (38\%) than Whites (25\%) were overweight.

Using the CATCH data, NHANES I (1971-1974), NHANES III (1991-1994), and the Bogalusa Heart Study (1991-1994), Dwyer et al. (2000) found that overweight was more prevalent among Blacks, Mexican Americans, and American Indian children compared to White children using weight percentiles for age and sex. Among the Pima 
Indians, the rate of overweight is considered to be the highest in the world with $50 \%$ of males and females having more than $95^{\text {th }}$ percentile of BMI (Krosnick, 2000). American Indian children 5-18 years old fall above the $85^{\text {th }}$ percentile BMI for age and sex (Broussard, Sugarman, \& Bachman-Carter et al., 1995). These studies on higher rates of obesity in Blacks and Indians are of particular importance, since Jamaica's population is comprised mainly of Blacks (91\%) followed by mixed ethnicities (7\%) and Indians (1\%).

\section{Measuring Obesity in Children}

Research showed that "one in four overweight children is already showing signs of type 2 diabetes (impaired glucose intolerance), with $60 \%$ already having one risk factor for heart disease" (Freedman, et al., 1999). However, it is difficult to measure obesity in children and adolescents since the timing and onset of puberty varies making it harder to set cut-off points (Singh et al., 2004; Hardy, Harrell \& Bell, 2004). Further, there is contradiction over the International Obesity Task Force (IOTF) and the World Health Organization (WHO) definitions for screening obesity among younger populations since these cut-off points were determined from populations from developed countries and do not represent the developing world.

The terms "overweight," and "at risk of overweight" have been suggested by the Centers for Disease Control (CDC) instead of "obesity" when referring to children and adolescents (American Obesity Association, 2006). However, the use of percentiles is suggested by The American Obesity Association with a BMI above the $85^{\text {th }}$ percentile for overweight and above $95^{\text {th }}$ percentile for obesity. The $95^{\text {th }}$ percentile corresponds to the adult BMI of $30 \mathrm{~kg} / \mathrm{m}^{2}$ which indicates obesity, whereas, the $85^{\text {th }}$ percentile corresponds 
to a BMI of $25 \mathrm{~kg} / \mathrm{m}^{2}$ which indicates overweight. The CDC suggests two levels of overweight; "children $>95^{\text {th }}$ BMI percentile for age and gender are considered overweight, and those $>$ BMI $85^{\text {th }}$ percentile are considered at risk for overweight."

The BMI for children is usually referred to as "BMI for age." The CDC suggests applying percentiles after the BMI has been calculated based on weight and height. Percentiles are suggested since they help to indicate the relative position of the child's BMI in relation to other children of the same age and sex (American Obesity Association, 2006). Age and gender are considered significant when determining cut-off points for children and adolescents since the amount of body fat changes with age. Girls tend to have more body fat than boys. See table 3 for the CDC BMI for age percentile.

Table 3

BMI for Gender and Age Percentile

Weight status category Percentile range

Underweight Less than the $5^{\text {th }}$ percentile

Healthy weight $\quad 5^{\text {th }}$ percentile up to the $85^{\text {th }}$ percentile

At risk of overweight $\quad 85^{\text {th }}$ to less than the $95^{\text {th }}$ percentile

Overweight Equal to or greater than the $95^{\text {th }}$ percentile

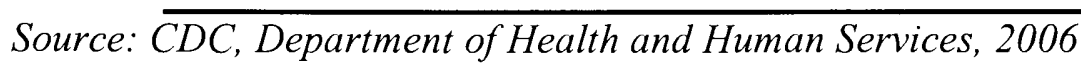

Measures used to determine childhood overweight must take into account developmental periods in children's lives when the BMI rises because of the growth spurts such as at age 4-6 and again during puberty (Dietz, 1997). In this study, adolescents ages 14-19 will be used. 


\section{Central Adiposity and Obesity}

It is becoming increasingly recognized that BMI might not be the best way of assessing overweight and obesity. The higher prevalence of diabetes among Indians may be related to their tendency to deposit fat in the abdominal area despite having lower BMI. Similar to higher BMI, abdominal fat or central adiposity is closely associated with hyperinsulinemia and insulin resistance (Ramachandran, 2004). According to Ramachandran (2004), "visceral fat increases the risk of diabetes and hyperlipidemia by favoring insulin resistance." Studies done in India on non-diabetic Indians revealed that insulin resistance was associated with subcutaneous fat. It is theorized that "increased risk posed by intra-abdominal fat for diabetes and other metabolic diseases may be related to the higher number of fat cells in the abdominal adipose tissue, higher blood flow, increased receptors for cortisol and testosterone and greater catecholamine induced lipolysis in comparison to subcutaneous adipose tissue" (Ramachandran, 2004).

The researcher further pointed out that there is increased influx of non-esterified fatty acids to the liver of abdominally obese individuals that may be related to the increase of insulin resistance and susceptibility to the metabolic syndrome. Arguments in support of upper body adiposity and relation to insulin resistance came from a study comparing non-diabetic Asian Indians and Mexican Americans (Ramachandran, Snehalatha, \& Viswanathan, et al., 1997). In this study, the Asian Indians had lower BMI than the Mexican Americans, but had greater upper body adiposity as measured by waistto-hip ratio which explains the high insulin resistance among Asian Indians despite having lower BMI. 
Some risk factors associated with central adiposity include glucose intolerance, hyperinsulinemia, hypertriglyceridemia, and hypertension (Ramachandran, 2004). These are also risk factors for cardiovascular diseases. Earlier studies on central adiposity and insulin resistance indicated that in Asian Indians a 0.04 unit increase in waist to hip ratio (WHR) resulted in a four-fold increase in diabetes (20\% in Asians compared to $5 \%$ in Europeans), also Indians had significantly higher post-glucose insulin levels (41 A $\mu / \mathrm{ml}$ vs. $19 \mathrm{~A} \mu / \mathrm{ml}$ ) higher triglycerides and lower HDL than Europeans (Mckeigue, et al., 1991). Further support on central adiposity, a risk factor for CVDs, T2DM, and hypertension comes from the International Task Force on Obesity (IOTF) which stated that "populations with low BMI such as Asians tend to have high levels of abdominal fat and are particularly prone to CVDs, T2DM and hypertension" (IOTF, 2006). Gopalan (1998) in a study on Asian Indians found that up to $20 \%$ of subjects, although not overweight, had central obesity, putting them at risk to CVDs, T2DM and hypertension.

Since central adiposity poses a risk for diabetes and insulin resistance, cut-off values have been determined for waist circumference, (WC) of $85 \mathrm{~cm}$ for men and $80 \mathrm{~cm}$ for women) and waist-to-hip ratio (WHR) of 0.89 and 0.81 for men and women respectively (Ramachandran, 2004). The use of WC instead of WHR has been recommended as an index of central adiposity since it shows an interaction with general adiposity at lower levels of BMI. In this study, waist circumference, WHR, and BMI were used to determine those at risk for T2DM and CVDs. See table 4 for genderspecific waist circumference for increased risk associated with metabolic complications in Caucasians. 
Table 4

Waist Circumference and Obesity-Associated Metabolic Complications

\begin{tabular}{lll}
\hline Gender & \multicolumn{2}{l}{ Risk of obesity-related metabolic complications } \\
\hline & Increased & Substantially Increased \\
\cline { 2 - 3 } Male & $\geq 94 \mathrm{~cm}$ & $\geq 102 \mathrm{~cm}$ \\
Female & $\geq 80 \mathrm{~cm}$ & $\geq 88 \mathrm{~cm}$ \\
\hline
\end{tabular}

\section{Clustering of Risk Factors for T2DM and CVDs}

Obesity in childhood and adolescence is associated with a number of clinical manifestations. The most common obesity-associated problems (dyslipidemias, and hyperinsulinemia) are found in more than half of the overweight children and adolescents. Research has shown that "up to $60 \%$ of overweight children had at least one cardiovascular risk factor such as high cholesterol, elevated insulin levels, and hypertension compared to $10 \%$ of children with healthy weight who had at least one risk factor"(Freedman, et al, 1999). Freedman et al. (1999) also found that up to $25 \%$ of overweight children had two or more risk factors. Overweight children and adolescents may have increased risks of morbidity and mortality in adulthood regardless of their adult weight status (Deckelbaum \& Williams, 2001). Research has shown that about one third of all severely obese adults were overweight as children (Whitaker, Wright, Seidel, et al., 1997).

The United States Surgeon General's Report (US Department of Health and Human Services, 2006) stated that "overweight adolescents have a $70 \%$ chance of becoming overweight or obese adults. This risk of becoming obese is $80 \%$ if one or more 
parents are overweight or obese. Obese adults are at increased risk of overall mortality and death from cardiovascular diseases, cancer, diabetes, and digestive diseases." The WHO has coined the term "metabolic syndrome" to represent the existence of T2DM and cardiovascular diseases because the two seem to go together (Weis, 2005). To receive a diagnosis of the metabolic syndrome, an individual must have at least three of five criteria: 1) elevated blood pressure, 2) high triglyceride level, 3) low HDL-cholesterol, 4) high fasting glucose and 5) central obesity (NCEP and ATP III Panel, 2006). This syndrome affects approximately $25 \%$ of US adults and predisposes one to atherosclerosis and diabetes (Weis, 2005). This syndrome may be present in about $30 \%$ of obese adolescents (Weis, 2005).

\section{Health, Social, and Economic Costs of Obesity}

Obesity threatens to reverse the remarkable changes of medical science of the $20^{\text {th }}$ century by decreasing life expectancy with its accompanying health-related problems of T2DM and CVDs (Daniels, 2006; Siepel, 2005). Obesity shortens the life span. Research on years of life lost due to obesity, shows that, White males lose 13 years, Black males 20 years, White females lose 8 years and Black females lose 5 years (Fountaine, Redden, \& Wang et al., 2003). Further support on years of life lost due to obesity comes from Murray \& Lopez (1994) in an Australian study. The authors found that the burden of disease associated with obesity and overweight increases in men 55-64 years and in women over age 55 years. After adjusting for confounding factors such as physical activity, this study still showed that in both sexes over $4 \%$ of total disability and life years lost were attributable to obesity and overweight. Death resulting from obesity 
is fast approaching that caused by smoking (Allison, Fountaine, \& Manson et al, 1999). Obesity has been found to increase the risk of diabetes. Ford et al. (1997) found that for every kilogram in weight increase, the risk for diabetes increases by $4.5 \%$.

Researchers have found that unhealthy weight gain increases the risk of heart disease in both men and women (Galanis, Harris, \& Sharp et al., 1998). It is estimated that "a gain of 10-20 pounds increases the risk of coronary heart disease to 1.25 and 1.6 times in women and men respectively" (Willet, Manson, Stampfer et al., 1995; \& Galanis, et al., 1998). As weight gain increases, the risks become greater. For example, increase of 22 pounds or over for men, and 44 pounds for women increase the risk of coronary heart disease to 1.75 and 2.65 respectively (Willet, et al., 1995; Galanis, et al., 1998). Some health risks associated with obesity among others, include premature death, heart disease, type 2 diabetes, stroke, sleep apnea, asthma, breathing problems, cancer (endometrial, colon, kidney, gallbladder and post-menopausal breast cancer), high blood cholesterol, menstrual irregularities, hirsutism (growth of excess body and facial hair), stress, incontinence, increased surgical risk, and depression (National Institute of Diabetes and Digestive and Kidney Diseases (NIDDK), 1996).

In addition to the physical and health problems associated with obesity, psychological and social consequences are also associated with obesity. For example, obese adolescents have lower college acceptance rates, lower desirability to employers, prospective attainment of lower social class, and increased risk for body dissatisfaction. Obese persons are usually stigmatized and discriminated against (Siepel, 2005). They experience low self-image, depression, anxiety, and overall lower quality of life than those with healthy weights. 
Obesity poses an economic burden on countries and governments through direct medical costs for hospital care, physician's services, medications, and indirect costs associated with obesity. Indirectly, costs due to obesity were calculated by looking at loss of productivity due to premature deaths, and restricted labor activities (Wolf \& Colditz, 1998). Using data from the 1988-1994 National Health Interview Survey (NHIS), Wolf and Colditz (1998) estimated that the economic cost of obesity in 1995 was $\$ 99.2$ billion. Of this amount, $\$ 51.64$ billion was attributed to medical cost, more than half of which went towards treating diabetes. Substantial amounts were also spent on treating coronary heart disease ( $\$ 16.97$ billion). In terms of indirect cost which is arrived at by extrapolating from labor days lost from premature deaths, disability and excess absenteeism, Wolf and Colditz (1998) found that up to $\$ 46.56$ billion were lost in indirect costs of obesity. Further support on the economic cost of obesity comes from Tucker (1998) who indicated that obese employees were 1.74 times more likely to be absent from work than those with healthy weights. The economic cost of obesity was estimated to increase from US\$99.2 billion in 1995 to $\$ 117$ billion in 2000 . Most of these obesityrelated costs are associated with type 2 diabetes, coronary heart diseases, and hypertension (Wolf \& Colditz, 1998). Although the current study did not calculate the economic cost of obesity and obesity-related health conditions, an understanding of the medical costs associated with obesity, is helpful in planning programs for the prevention of T2DM and CVD; conditions related to obesity. 
Acanthosis Nigricans, Obesity, Type 2 Diabetes, and Cardiovascular Diseases

Acanthosis Nigricans is manifested by dark, raised skin in areas such as the axilla, neck, knuckles, elbows, and knees and is believed to result from hyperinsulinemia. Acanthosis Nigricans is manifested by a darkening of the skin, velvety plaques of body folds and is most commonly seen around the neckline or in the axilla in children who have greater body weight and fat mass, higher serum insulin levels and lower insulin sensitivity (McGillis-Bindler, \& Bruya, 2005, Burke, Hale, \& Hazuda et al., 1999). Acanthosis Nigricans is becoming more prevalent among obese patients and those with type 2 diabetes mellitus (Burke et al., 1999). Subjects with AN were also at risk for atherosclerotic cardiovascular diseases (Katz, \& Feldman, 2006).

The presence of AN is a marker for the risk factor of hyperinsulinemia. Hyperinsulinemia leads to binding of insulin-like growth factor (IGF) receptors on keratinocytes and fibroblasts and ultimately hyperplasia of the skin (Cruz, \& Hud, 1992). It is believed that obesity underlies this insulin resistance. High fasting blood insulin levels confirm the presence of hyperinsulinemia. Persons with hyperinsulinemia are often able to maintain glucose homeostasis despite the presence of insulin resistance. They exhibit normal blood glucose despite the presence of T2DM especially in individuals over age 40 . Therefore testing of blood insulin levels may help to improve the condition. Hyperinsulinemia with normal blood glucose is an indicator of prediabetes.

Insulin resistance (IR), that is, resistance to insulin mediated glucose disposal, is present in T2DM and has been linked to cardiovascular diseases (Reaven, 1988; Laakso, 1996; Stern, 1996). Reaven, (1988) postulated that IR may underlie other disorders such 
as hypertension, dyslipidemia, (high triglyceride and low HDL levels), impaired glucose tolerance, and coronary heart disease. There has been an association with diabetes mellitus and atherosclerotic cardiovascular disease, therefore, control of blood glucose can help to control coronary and carotid disease (Reaven, 1988). Obesity causes insulin resistance which is associated with AN (Reaven, 1988), therefore intervention is needed to correct the weight loss and provide dietary measures to help correct the problems. Exercise and improved diet can help to lower insulin levels and can improve AN.

Obesity and Physical Activity, and Relationship to T2DM and CVDs

Physical activity "differs from exercise and refers to any movement produced by muscular contractions that burn extra calories" (Harvard School of Public Health, 2006). Individuals who live a sedentary lifestyle are more likely to become overweight and develop chronic diseases. Some benefits of physical activities according to the US Surgeon General's Report are; improved longer and healthier life, protection from heart diseases and its precursors (high blood pressure and high cholesterol), protection against certain cancers, prevention or control of type 2 diabetes and control weight among other benefits." Earlier research found a positive association between glycemic control $(\mathrm{HbA1c})$ and aerobic fitness or reported physical activity among children with type 1 diabetes (Sackey \& Jefferson, 1996; \& Horgan, 2005). However, very little is known about the impact of regular exercise on metabolic control in adolescents with type 2 diabetes. Studies have shown improvement in high-density lipoprotein levels and endothelial function in obese insulin-resistant adolescents (Kelly, Wetzseteon, Kaiser, et al., 2004). 
Simple exercise such as walking briskly for 20 minutes per day can help reduce the chances of having a heart attack or stroke, developing diabetes or dying prematurely. At least 30 minutes of moderate intensity physical activity is recommended each day by the CDC, US Surgeon General, and the American College of Sports Medicine (Pate, Pratt, \& Blair, 1995). The CDC estimates that physically active persons can help to cut the direct medical costs in the US by as much as $\$ 70$ billion yearly (Pratt, Marcera $\&$ Wang, 2000).

Measurement of physical activity is more accurately done by Physical Activity Questionnaires (PAQs) which cover day-day individual activities, inexpensive, requires little labor, and are useful in epidemiological studies. These recorded activities are then measured in terms of energy expenditure. The energy costs of physical activities are then expressed in metabolic equivalents (MET) units (Kwan, Woo, \& Kwok, 2004). One Met is estimated to be $3.5 \mathrm{ml} / \mathrm{min} / \mathrm{kg}$ resting oxygen consumption $\left(\mathrm{VO}_{2}\right.$ resting $)$ for all age and gender groups unlike the BMR which closely resembles the MET but varies by age and gender. The CDC and American College of Sports Medicine (ACSM) have compiled a comprehensive list of physical activities based on level of intensity of such activities.

\section{Obesity, T2DM, CVDs, and Food Intake}

The macronutrient composition of the diet and relation to diabetes, CVDs and obesity is widely studied. In general, the types of fats consumed rather than the amount (g) shows health risks compared to health benefits. Saturated fats are associated with CVDs whereas intakes of types of fatty acids such as the omega- 3 fatty acids seem to have a protective property $(\mathrm{Hu}, 2000$; Howard, 2000; Mayer-Davis, 2000). Research is 
lacking on the relationship between the consumption of dietary fat and T2DM, but fats contribute to obesity which is a major risk factor of T2DM (Hu, 2000). Arguments in favor of carbohydrates and T2DM relate to the glycemic index of the food eaten. Types of carbohydrates with low ability to raise blood glucose levels are recommended in the prevention of T2DM.

Research has shown that dietary fiber and its relation to glycemic load may have a beneficial effect on CVDs (Hu, 2000; Pereira \& Liu, 2005). The association between dietary fiber and glycemic load and effects on CVDs was stronger among overweight individuals than non-overweight (Pereira \& Liu, 2005). Consumption of whole grain has also been associated with reduced risk of CVDs among men and women (Loke, 2005; Liu, 2002; Gross, Li, \& Ford et al:, 2004). Epidemiological studies have shown that diets rich in high-fiber grains lower the risk of CVDs and T2DM (Liu, 2002; Salmeron, Manson, \& Stampfer et al., 1997). Women who consumed foods with high glycemic load (GL) and had low cereal fiber intake were found to be 2.3 times more likely to develop T2DM (Salmeron et al., 1997). Intakes of refined carbohydrates have been associated with CVDs since carbohydrates induce hypertriglyceridemia by enhancing hepatic synthesis of very low density lipoproteins (VLDL) and reducing lipoprotein lipase activity (Liu et al., 2001)

Increased consumption of refined carbohydrates is associated with increased rates of T2DM in the USA (Gross, et al., 2004). In a study comparing the USDA per capita nutrient consumption of USA residents between 1907-1997 with the CDC's prevalence of T2DM, significant correlations were found between changes in T2DM and changes in dietary fat $(\mathrm{r}=.84, \mathrm{p}<.001)$, carbohydrates $(\mathrm{r}=.55, \mathrm{p}<.001)$, protein $(\mathrm{r}=.71, \mathrm{p}<.001)$, 
fiber $(r=-.16, p<.001)$, corn syrup $(r=.83, p<.001)$ and total energy $(r=.75, p<$ .001). The authors concluded that 'increased intakes of refined carbohydrates (corn syrup) along with decrease in fiber intake paralleled the upward trend in diabetes prevalence" (Gross et al., 2004).

Consumption of macronutrient is important to this study in relation to their association with overweight and consequently to BMI which are also risk factors of T2DM and CVDs. Excess energy intake leading to overweight may be related to low intakes of fiber. High intake of fibers reduced energy intake by decreasing energy density and also enhanced satiety (Howarth, Terry \& Huang et al., 2005; Howarth, Saltzman \& Roberts, 2001; Yao \& Roberts, 2001). High fiber intake also correlated with low fat, low energy density and low glycemic index (Howarth, et al., 2001).

\section{Comparison of Finger Prick versus Venous Blood Testing}

Differences have been noted between whole blood capillary glucose and venous plasma glucose with values for capillary whole blood being higher than venous blood. The concentration of water is approximately $11 \%$ higher in plasma than whole blood therefore glucose concentrations in plasma are $11 \%$ lower than whole blood (Sacks et al., 2002). Despite these limitations of capillary whole blood, they are useful in making quick diagnoses for persons who would normally have to be sent to a laboratory. An advantage of this method is it allows immediate feedback to the patient when tests are performed on location. The test is also less costly than laboratory testing. The cost of treating diabetes due to its debilitating conditions from hypoglycemia and ketoacidosis and CVDs for US residents was estimated at US\$98 billion in 1992 (Sacks, 
et al., 2002). Further, in one US based study, FBG was shown to be comparable with FPG in diagnosing T2DM among 40-69 year olds without known diabetes. Of the 1,028 persons screened, diabetes was confirmed in 22 by FBG or FPG and 14 were confirmed by both the FBG and FPG (Sandbaek, Lauritzen, Borch-Johnsen et al., 2005). The prevalence of confirmed diabetes was lower (2.1\%) compared to $3.3 \%$ for unconfirmed diabetes in this study (Sandbaek, et al., 2005).

Variability between blood glucose measured using self-monitoring devices and laboratory testing of plasma glucose showing a difference of less than $20 \%$ between capillary blood glucose and simultaneous venous FPG levels at FBG levels $>4.2 \mathrm{mmol} / \mathrm{L}$ have been noted (Sacks et al.,2002). The International Federation of Clinical Chemistry (IFCC) recommended that all blood glucose monitoring systems should give a comparable plasma result. On an average, results that are referenced to plasma should be approximately $11 \%$ lower than results referenced to whole blood (D’Orazio, Burnett, \& Fogh-Andersen et al., 2005). The Accu Chek Advantage used in this study aligns closely with this $11 \%$ recommendation (Roche Diagnostics, New Zealand).

Total Cholesterol (TC)

Cardiovascular diseases are the leading cause of death in the world. Early monitoring of cholesterol can help to reduce the global burden of treating CVDs. Development of CVDs usually starts in the earlier years and progresses to clinically significant lesions in young adulthood (Gungor, \& Arslanian, 2005; Steinberger et al., 2003). Cholesterol testing in children and adolescents is needed since fatty streaks associated with atherosclerosis are now found in the aortas of children as young as three 
years old (McGillis-Bindler, \& Bruya, 2000). High cholesterol levels have been associated with premature CVDs (Frick, Elso, \& Haspa et al. 1987; Brensike, Levy, \& Kelsey et al., 1984). High-density lipoproteins (HDLs) are associated with protection against heart diseases whereas low-density lipoproteins (LDLs) are considered risk factors for the development of chronic diseases (NCEP, 1995). Subjects with type 2 diabetes tend to have low HDL-C and high levels of triglycerides. The ADA recommends lowering LDL and total cholesterol as well as monitoring blood pressure for persons with diabetes. Cholesterol screening is recommended and many screening tests are now performed in physicians' offices or clinics.

Earlier research showed that point-of-care testing for TC shows lower precision than standardized laboratory testing but may be used in "resource-poor settings" (Rotchford, Machattie \& Gills, 2002). Measurement of TC alone is not as accurate as using the TC/HDL-C ratio. Also capillary testing of TC is not as accurate as venous testing (Dipeolu, 2006). Research shows that EDTA anti-coagulated venous plasma measurements are approximately $3 \%$ lower than yields from venous serum measurements. Therefore, the NCEP advises adding 3\% to venous plasma results to allow for comparisons with NCEP cutoffs (Greenland, Bowley, \& Melklejohn et al., 1990).

Studies which compared capillary versus venous testing of TC (Bowden, Kingery \& Rust, 2004; Kafonek, Donnovan, \& Lovejoy, 1992; Costanza, Wolf \& James et al., 2005 ) showed conflicting results. Use of capillary whole blood classified more subjects as low risk for TC compared to venous method (Bowden et al, 2004). Whereas Kafonek et al. (1992) found similar results for lipids measured by finger prick and venous blood 
samples, another study (Costanza et al., 2005) found small bias between venous and capillary whole blood samples of TC.

Glycated Hemoglobin

Hemoglobin is the oxygen-carrying pigment that gives blood its red color. Approximately $90 \%$ of the hemoglobin is classified as hemoglobin A. The "A" stands for adult type. One chemical component accounts for $92 \%$ of the hemoglobin and the remaining $8 \%$ is further divided into four components called A1c, A1b, A1a1, and A1a2. Hemoglobin A1c (HbA1c) is a minor component to which the glucose molecule is bound. HbAlc is also called glycated hemoglobin or glucosylated hemoglobin.

The purpose of the HbAlc test is to determine how much glucose remains on the hemoglobin molecule and which helps to determine the plasma glucose concentration over time. The principle is based on the 120 day life-span of the red blood cells. During this time the glucose molecule binds to the hemoglobin and forms glycated hemoglobin. Once the hemoglobin becomes glycated it remains glycated. Therefore a build-up of glycated hemoglobin within the cells reflects the average level of glucose to which the cell has been exposed for its life-span. The level of $\mathrm{HbAlc}$ is proportional to the average blood glucose concentration during the three months period.

The Diabetes Control and Complications Trial (DCCT) and the United Kingdom Prospective Diabetes Study (UKPD) provided reference values for HbA1c. The DCCT study involved 1,400 persons with type 1 diabetes conducted between 1983-1993. After 6.5 years, persons in the intensive treatment group (monitored glucose 3-4 times daily, used insulin pump or injections) attained an average $\mathrm{HbA} 1 \mathrm{c}$ of $7.3 \%$ while those in the 
conventional therapy group was $9.1 \%$. In the UKPDS study of 4500 persons with type 2 diabetes those treated with a diet regimen had $\mathrm{HbAl}$ c levels of $7.9 \%$ compared to the intensive regimen with 7.0\% (Nakamoto, 2006).

Achievement of a $6.5 \%$ reading for $\mathrm{HbAlc}$ indicated blood glucose that is under control. It is possible to have normal $\mathrm{HbAlc}>8 \%$ and have blood glucose of $120 \mathrm{mg} / \mathrm{dL}$ if the blood glucose is tested once per day. However, at other times of the day the blood glucose may be above $120 \mathrm{mg} / \mathrm{dL}$. It must be noted that to every $1 \%$ increase of $\mathrm{HbA} 1 \mathrm{c}$ there is a $35 \mathrm{mg} / \mathrm{dL}$ or $1.95 \mathrm{mmol} / \mathrm{L}$ increase in mean plasma glucose.

Lower finger prick values of $\mathrm{HbA} 1 \mathrm{c}$ compared to venous blood were found in 147 diabetic patients age 18 years and older tested at two family practice centers which routinely use $\mathrm{HbA} 1 \mathrm{c}$ analysis at an outside laboratory for venipuncture samples. The aim of the study was to determine if same-day-visit testing of $\mathrm{HbAlc}$ (using capillary blood finger prick samples) could be used in these health care centers for more efficient patient care and improved diabetes management (Schwartz, Monsur \& Bartoces et al., 2005).

In another study of 333 outpatients screened for diabetes mellitus using $\mathrm{HbA} 1 \mathrm{c}$ measures, fasting plasma glucose and a two hour post-load oral glucose tolerance test, $\mathrm{HbAlc}$ had sensitivity of $60 \%$ compared to FPG and OGTT with $52 \%$ and $40.0 \%$ respectively and specificities of $90.9,98.7$ and 99.4 percent for the three measures. The predictive values for a positive diagnosis were $34.9 \%, 76.5 \%$ and $83.3 \%$ for the three tests (Simon, Coignet, Thibult et al., 1985). Specificity refers to "the proportion of people without the disease who have a negative test result and sensitivity means the proportion of people with the disease who have a positive test result." The results indicated that HbA1c had higher sensitivity, but lower specificity than the FPG and OGTT. From a 
public health point of view tests with high specificity and predictive values are better than those with high sensitivity but poor specificity (Simon, et al., 1985).

The normal $\mathrm{HbA} 1 \mathrm{c}$ range for a healthy person is $4-5.9 \%$. The International Diabetes Federation and American College of Endocrinology recommends that $\mathrm{HbA1c}$ values below 6.5\% whereas the American Diabetes Association extends this range to $7 \%$. Ranges above 7\% represent poor glucose control (Nakamota, 2006).

\section{Summary}

Obesity is the main risk factor for T2DM and CVDs in children and adolescents and a predictor of adult obesity. As the prevalence of pediatric obesity increases around the world in developed and developing countries, the burden of life-style diseases is expected to increase, overburdening the public health systems in countries with limited health resources. More information is needed on the risk factors for these diseases to assist in the development of effective programs to prevent the morbidity and mortality related to obesity, T2DM, and CVDs, and to identify preventable risk factors early in children and adolescents, such as high BMI, socio-economic factors, high BP, presence of AN, dietary distortions, and low physical activity. A suitable and economical method of blood testing is needed for a resource-poor country such as Jamaica. Finger prick versus venous testing was performed. The literature shows conflicting results for finger prick versus venous testing (the gold standard), however, few studies showed comparable results for both method. Using the information presented in this chapter, this study was designed to assess these critical emergent problems. 
Summary of Literature on T2DM and CVDs among Adolescents

\begin{tabular}{lll}
\hline Reference & Areas Assessed & Population \\
\hline $\begin{array}{l}\text { Headley et al., } \\
2004\end{array}$ & $\begin{array}{l}\text { Prevalence of obesity } \\
\text { among US children } \\
\text { and adolescents }\end{array}$ & $\begin{array}{l}\text { Survey of 4018 children, } \\
\text { Data from NHANES } \\
1999-2000 \text { and 4258 } \\
\text { children in 2001-2002 }\end{array}$ \\
Gungor et al., & $\begin{array}{l}\text { Obesity and related } \\
\text { chronic diseases }\end{array}$ & $\begin{array}{l}\text { Clinical study of 20 } \\
\text { adolescents with T2DM, } \\
\text { 2005 normal weight and 20 } \\
\text { obese adolescents as } \\
\text { controls. }\end{array}$
\end{tabular}

Main Findings

Increase in

overweight

among children and

adolescents was

$15.0 \%$ in children

$6-19$ years in 1999

-2000 and $16.5 \%$

in 2001-2002.

Pulse wave velocity

(PWV) higher in

subjects with

T2DM and the overweight group.

PWV significantly

correlated with

glucose, HbAlc,

BMI, IR and C-

reactive protein.

Overweight

subjects are at

higher risk to CVDs

than normal weight

counterparts

\begin{tabular}{lcc}
\hline T2DM $=$ type 2 diabetes & PWV $=$ Pulse Wave Velocity & CVDS= cardiovascular diseases \\
NHANES $=$ National Health and Nutrition Education Survey & IR $=$ Insulin resistance
\end{tabular}




\begin{tabular}{|c|c|c|c|c|}
\hline Reference & Areas Assessed & Population & Main Findings & Suggestions/Future Needs \\
\hline $\begin{array}{l}\text { Borders et al., } \\
2006\end{array}$ & $\begin{array}{l}\text { Gender specific } \\
\text { disparities in obesity } \\
\text { by place of esidence, } \\
\text { SES, ethnicity }\end{array}$ & $\begin{array}{l}\text { Survey data from the } \\
2003 \text { Behavioral Risk } \\
\text { Factor Surveillance } \\
\text { System (BRFSS) for the } \\
\text { State of Texas. }\end{array}$ & $\begin{array}{l}\text { Hispanic or Black } \\
\text { females were more at } \\
\text { risk for obesity. } \\
\text { Females with high } \\
\text { household income } \\
\text { were less at risk to } \\
\text { obesity. Subjects in } \\
\text { rural areas were more } \\
\text { at risk for obesity } \\
(\mathrm{p}<.001) \text {. }\end{array}$ & $\begin{array}{l}\text { Policies and programs } \\
\text { needed to target males } \\
\text { and females residing } \\
\text { in rural areas. Collect } \\
\text { research evidence on these } \\
\text { demographics for the } \\
\text { Jamaican population. }\end{array}$ \\
\hline $\begin{array}{l}\text { Seidell et al., } \\
2001\end{array}$ & $\begin{array}{l}\text { Comparison of WC } \\
\text { and WHR as } \\
\text { measures of adiposity } \\
\text { and risk for CVDs. }\end{array}$ & $\begin{array}{l}313 \text { men and } 382 \text { women } \\
\text { in Quebec }>18 \text { years. }\end{array}$ & $\begin{array}{l}\text { Large WC associated } \\
\text { with high LDL-C in } \\
\text { women. Lower } \\
\text { correlations of the } \\
\text { risk factors for BMI } \\
\text { than for WC and } \\
\text { WHR. }\end{array}$ & $\begin{array}{l}\text { Identify similar trends } \\
\text { in the Jamaican } \\
\text { population for future } \\
\text { planning to reduce } \\
\text { overweight and obesity } \\
\text { and lower WC. }\end{array}$ \\
\hline $\begin{array}{l}\text { Kobaissi et al., } \\
2004\end{array}$ & $\begin{array}{l}\text { Overweight, AN and } \\
\text { IR as risk factors for } \\
\text { T2DM among } \\
\text { children. }\end{array}$ & $\begin{array}{l}131 \text { overweight Hispanic } \\
\text { children ages } 8-13 \text { years. }\end{array}$ & $\begin{array}{l}\text { AN is an independent } \\
\text { predictor of IR in } \\
\text { overweight Hispanic } \\
\text { children, but BMI } \\
\text { remains the primary } \\
\text { determinant of IR. }\end{array}$ & $\begin{array}{l}\text { Conduct study on AN } \\
\text { and IR in this population. } \\
\text { No evidence of this } \\
\text { been done before } \\
\text { in that group. Education } \\
\text { needed on prevention } \\
\text { of AN and improving IR. }\end{array}$ \\
\hline $\begin{array}{l}\text { SES = Socio-ec } \\
\text { IR = Insulin res } \\
\text { PA = physical a }\end{array}$ & $\begin{array}{l}\mathrm{AN}= \\
\mathrm{WHI} \\
\mathrm{BMI}\end{array}$ & $\begin{array}{l}\text { Acanthosis Nigricans } \\
=\text { waist-to-hip ratio } \\
=\text { Body mass index }\end{array}$ & $\begin{array}{l}\text { WC }=\text { waist circum } \\
\text { CVDS }=\text { cardiovasc } \\
\text { LDL-C }=\text { low densi }\end{array}$ & $\begin{array}{l}\text { erence } \\
\text { lar disease } \\
\text { lipoprotein }\end{array}$ \\
\hline
\end{tabular}




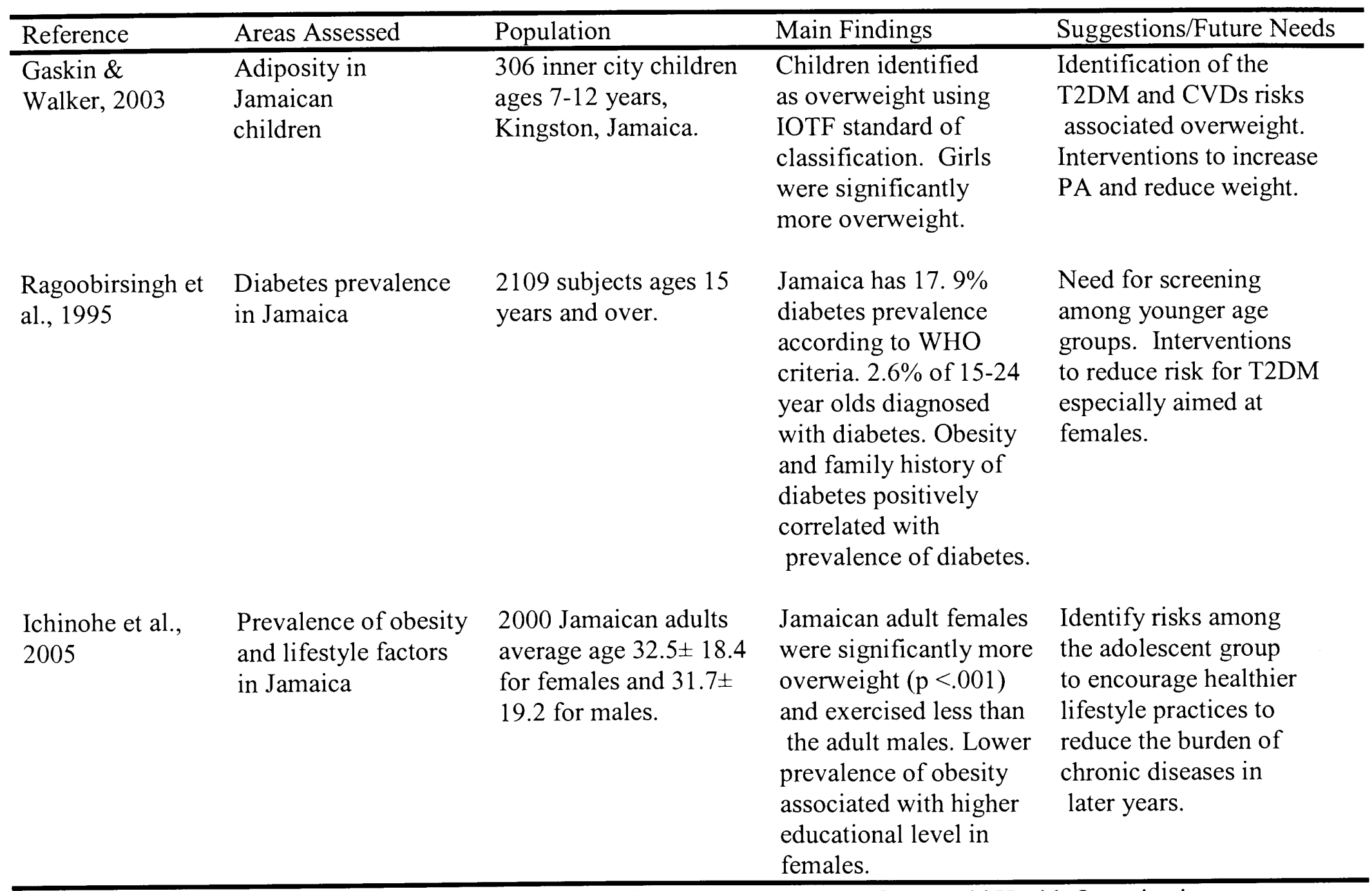

$\mathrm{T} 2 \mathrm{DM}=$ type 2 diabetes $\quad$ IOTF $=$ International Obesity Task Force $\quad$ WHO $=$ World Health Organization 


\begin{tabular}{|c|c|c|c|c|}
\hline Reference & Areas Assessed & Population & Main Findings & Suggestions/Future Needs \\
\hline $\begin{array}{l}\text { Pan \& Pratt, } \\
2008\end{array}$ & $\begin{array}{l}\text { Metabolic syndrome } \\
\& \text { association w/ PA } \\
\text { and diet }\end{array}$ & $\begin{array}{l}4,450 \text { US children and } \\
\text { adolescents ages } 12-19 \\
\text { years. }\end{array}$ & $\begin{array}{l}\text { Prevalence of } 3.5 \% \\
\text { METS. Males } \\
\text { significantly more at } \\
\text { risk to the METS than } \\
\text { females }(\mathrm{p}<.001 \text { ). } \\
\text { METS was } 16 \text { times } \\
\text { higher among } \\
\text { overweight adolescents } \\
\text { with BMI } \geq 95^{\text {th }} \\
\text { percentile. }\end{array}$ & $\begin{array}{l}\text { Identification of the METS } \\
\text { among Jamaican } \\
\text { adolescents using a } \\
\text { modified version of the } \\
\text { NCEP ATTP III guidelines. } \\
\text { Data can be used to plan } \\
\text { interventions to increase } \\
\text { PA, reduce weight, and } \\
\text { select healthy food choices. }\end{array}$ \\
\hline
\end{tabular}

Weis et al., 2004 Obesity and the METS in children and adolescents.
439 obese, 31

overweight, and 20

non-obese children

and adolescents ages

4-20 years.
Prevalence of METS increased with severity of obesity. Obese subjects were 1.5 times more likely to be classified with the METS.
Assessment of the risk factors for the METS among overweight Jamaican adolescents. Interventions needed to reduce biomarkers of T2DM and CVDs which are found in children and adolescents.

\footnotetext{
METS = metabolic syndrome

CVDS $=$ cardiovascular disease

BMI = body mass index

T2DM = type 2 diabetes

ATTP III = Adult Treatment Panel III
} 


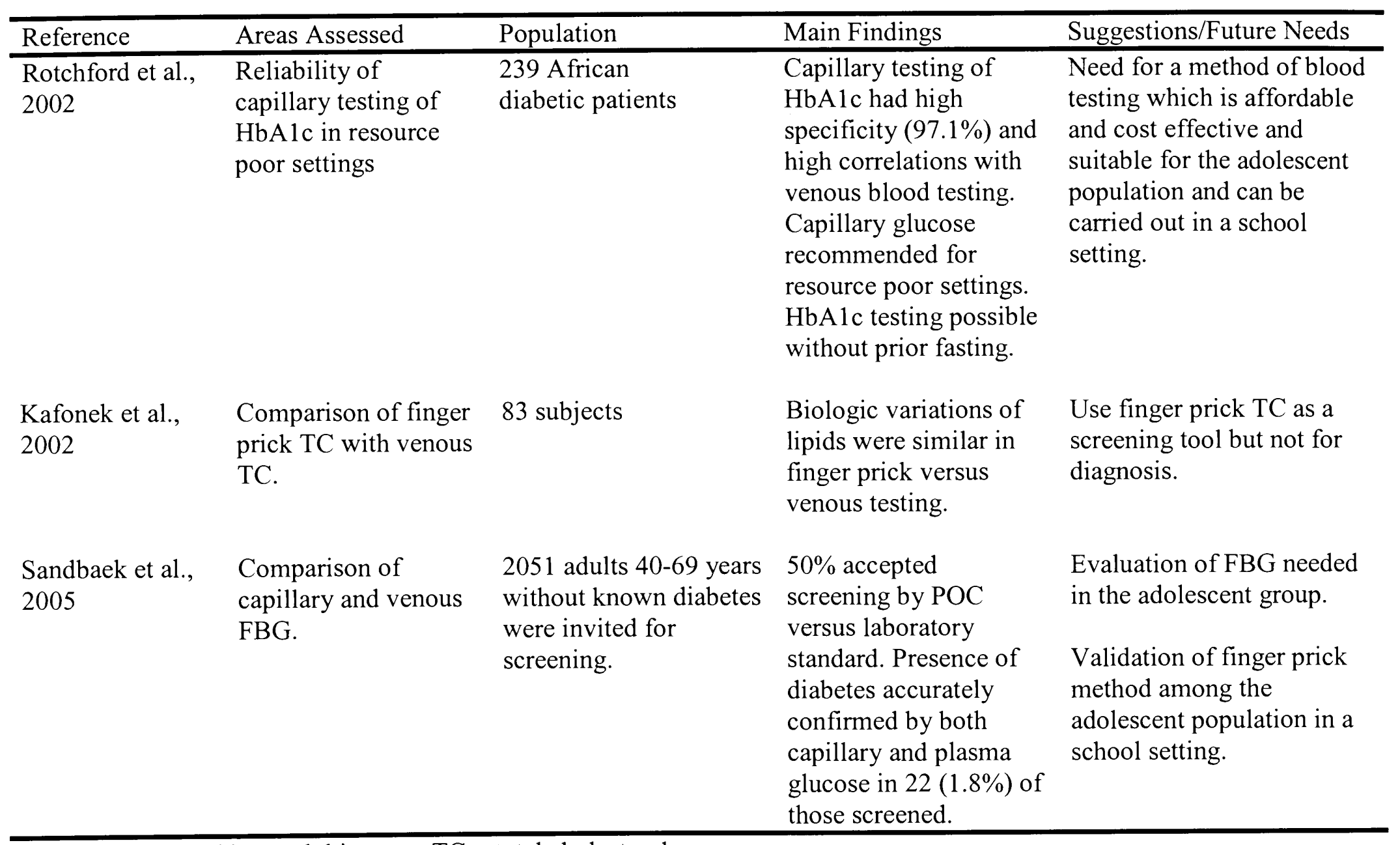

$\begin{array}{ll}\text { HbAlc }=\text { glycated hemoglobin } & \mathrm{TC}=\text { total cholesterol } \\ \text { POC }=\text { Point-of-care } & \text { FBG }=\text { Fasting blood glucose }\end{array}$


CHAPTER III

\section{METHODS AND DESIGN}

\section{Research Design}

The study was a non-experimental descriptive epidemiological study design which involved screening for risk factors of type 2 diabetes (T2DM) and cardiovascular diseases (CVDs) such as overweight and obesity, high levels of fasting blood glucose (FBG), glycated hemoglobin (HbA1c), total cholesterol (TC), blood pressure (BP), waist circumference (WC), waist to hip ratio (WHR), presence of Acanthosis Nigricans (AN), low physical activity (PA) levels, family history of overweight, T2DM, CVDs, and poor dietary intake of 276 adolescent students in selected high schools in Jamaica. A subsample of 59 randomly selected students was examined to compare capillary whole blood versus venous blood samples of $\mathrm{FBG}, \mathrm{HbAlc}$, and $\mathrm{TC}$ in the validation of the finger prick method for early screening of risk factors for T2DM and CVDs in a school setting.

\section{Study Population}

The study population comprised of students ages 14-19 years from the (rural and urban) secondary schools on the island of Jamaica. Approximately $28 \%$ of Jamaicans fall within the age group of $10-24$ years, with $50 \%$ living in the rural areas, and with more youths living in rural than urban areas (National Center for Youth Development, Ministry of Education, Youth and Culture, Jamaica, 2002).

Research has shown a preponderance of evidence that more Jamaican females than males were overweight (Gaskin \& Walker, 2003; Ichinohe, Mita, Saito et al., 2005). Data on school enrollment in Jamaica shows that up to age 16 years about equal numbers 
of males and females are enrolled in secondary schools but females predominate after age 16 (National Center for Youth Development, Ministry of Education, Youth and Culture, Jamaica, 2002).

All ethnicities were included since research has also shown that the prevalence of T2DM was higher among ethnic minorities (African Americans, Native Americans, Hispanics (Siepel, 2005; McKnight-Menci et al., 2005) in US based research. Another study showed increasing incidence of both obesity and T2DM among Asian children (Vikram, et al., 2005) and since Jamaica's population consists of approximately 91\% Blacks of African and 1\% of East Indian ancestry, the same dynamics are expected in this study population. Inter-racial marriages are also common and it is likely that the sample will also comprise mixed ethnicities. Data on Jamaica's population show a presence of $7 \%$ being of mixed ethnicities (Jamaica Facts and Figures, 2006). Most of the Indian population lives in the parishes of Westmoreland, Clarendon, and St Mary. The parish of Clarendon was selected to obtain a proportionate representation of this ethnic group.

Approximately $100 \%$ of children under the age of 14 years and $83 \%$ of children aged 15-16 years are enrolled in a school in Jamaica. It is also reported that up to $95 \%$ of children progress to secondary schools (National Center Youth Development, Ministry of Education, Youth and Culture, Jamaica, 2002). Therefore it was likely to find a representative sample from the secondary school population by selecting students from the different parishes. 


\section{Recruitment and Sampling}

Subjects were selected through purposive and stratified sampling from the 14 parishes on the island. The fourteen parishes were grouped into six main regions for administrative purposes by the Ministry of Education, Youth, and Culture. Of the six main regions, regions one, two, and six were selected to give a representative sampling for ethnicities, rural and urban locations and socio-economic status (SES). Region one consisted of the parishes of Kingston, St. Andrew, and part of St. Thomas. This region hosts the major urban areas of the island with Kingston being the island's capital city. The parish of St. Thomas and parts of St. Andrew however classified as rural areas. Region two consisted of St. Mary, Portland and parts of St. Thomas which classified as rural areas. Region six consisted of the parishes of Clarendon and St. Catherine and included both rural and urban locations.

Three regions were selected through cluster sampling based on ethnicity, SES, rural/urban location, and cost-effectiveness in accessing these schools. A list of all schools within each parish was made using the Ministry of Education's publication on school profiles. This list of secondary schools was further stratified by school type (traditional and non-traditional). Representations of all income levels and SES were ascertained by selecting the traditional high schools which typically cater to the middle and upper class families, and by having representation from the other new high and comprehensive high schools in the surrounding areas. Schools were then randomly selected from each parish and school type by placing the names of all traditional high schools in that parish in a hat and drawing one school. The non-traditional high school was selected in a similar manner. Two schools were randomly selected for each of the 
five parishes chosen to represent the two school types making a total of 10 schools. See Table 6 for the breakdown of the population by regions, parishes, school type and location. Selection of study subjects by regions, parishes, school type and location represented the following sub-groups; gender (male, female), ethnicity (Blacks, Indians, Mixed), SES (high, medium, low) and location (rural, urban).

Table 6

Breakdown of Population by Regions, Parishes, School Type and Location $(N=300)$

\begin{tabular}{lllll}
\hline Regions & Parishes & School Type & Location & Subjects \\
\hline 1 & Kingston & Traditional & Urban & 30 \\
& & Non-Traditional & Urban & 30 \\
& St. Andrew & Traditional & Urban & 30 \\
& & Non-Traditional & Urban & 30 \\
& St. Thomas & Traditional & Rural & 30 \\
& & Non-Traditional & Rural & 30 \\
6 & Clarendon & Traditional & Rural & 30 \\
& & Non-Traditional & Rural & 30 \\
& & Traditional & Urban & 30 \\
& & Non-Traditional & Rural & 30 \\
\hline
\end{tabular}

Subjects were selected from grade levels (9-11) to represent the age group 14-19 years. Thirty students from each of the ten schools were selected. A list of all students in 
grades 9-12 was obtained from the principal of each school. The students were stratified by grade level and gender and each student assigned a number. Students were then selected from each grade level/gender within each school by randomly selecting five numbers per grade and gender and matching those numbers to the names on the list.

Sixty subjects were also randomly selected from the original sample of 300 for validation of venipuncture screening. This selection was done by assigning an ID number to each of the 300 students. The numbers were entered into Microsoft Excel and 6 ID numbers were randomly selected from each school making a total of 60 .

Inclusion Criteria

The study included Jamaican adolescent ages 14-19 years who attended secondary schools on the island. Only those students whose parents gave written consent and who themselves signed an assent form were included in the study. Both normal and overweight adolescents were included in the study. Only subjects who weighed over 110 lbs and were in apparently good health had their blood drawn by venipuncture.

\section{Exclusion Criteria}

Subjects were excluded from the study if they were on medications known to alter blood pressure, glucose or lipid metabolism, and if they had known eating disorders.

\section{Calculation of Sample Size}

Correlations, chi square tests, multiple and logistic regressions were carried out for hypotheses testing. Using a multiple linear regression with six predictor variables for 
risk factors of T2DM and CVDS, a sample size of 300 was sufficient to yield 99 percent power to detect an $\mathrm{R}$ squared of .10 versus 0 using an F-test with a significance level of .05 (Faul, \& Erdfelder, 1992).

The following formula was used to calculate the 95 percent confidence interval for proportion of overweight Jamaican adolescents. The increase in childhood T2DM is linked to the growing rates of obesity among children and adolescents (Daniels, 2006; Choudbury, 2005; Reinehr, 2005; Steinberger et al., 2001) and T2DM is among the cluster of cardiovascular disease (CVDs) risk factors such as hypertension, and dyslipidemia (Sacks, et al., 2002). Gaskin and Walker (2003) surveyed 306 Jamaican children aged 7-8 and again at follow-up 11-12 years old in comparing different standards for determining overweight among Jamaican children. Results indicated an increase in overweight from $3.5 \%$ - to $9.5 \%$ over the follow-up period. Therefore, a projected proportion of overweight of 10\% among Jamaican adolescents, ages 14-19 years was used in calculating sample size.

$$
\mathrm{n}=\frac{\mathrm{z}^{2} \times \mathrm{p}(1-\mathrm{p})}{\mathrm{m}^{2}}
$$

$\mathrm{n}=$ required sample size

$\mathrm{z}=1.96$ (standard normal value for $95 \% \mathrm{CI}$ )

$\mathrm{p}=$ estimated prevalence of overweight $(10 \%$ or 0.10$)$

$\mathrm{m}=$ margin of error at $5 \%$ (standard value of 0.05 )

$$
\mathrm{n}=\frac{1.96^{2} \times \cdot 10(1-.10)}{0.05^{2}}=138
$$

The sample size of 138 was adequate for a research design that uses simple random sample selection. However, in this study, cluster sampling technique was used to ensure adequate representation of ethnicity, rural and urban locations and SES. To correct for 
the difference due to design effect, the sample was multiplied by 2 (FAO, 1990;

Magnani, 1997 \& UNICEF, 1995):

$$
\mathrm{n}=138 \times 2=276 \text {. }
$$

To allow for errors in recording or other contingencies, a further $5 \%$ of the sample was added;

$$
\mathrm{n}=276 \times .05=13.8
$$

The sample size was $276+14=290$. To allow for attrition the researcher added a further 10 study subjects making a total sample size of $300 / 10=30$ students per school. The total sample size for the study was therefore estimated at $\mathrm{N}=300$. That was, 30 students from each of the ten schools.

For the comparison testing of finger prick capillary whole blood and fasting venous plasma (hypothesis three) 60 students were randomly selected from the main study population. The power analysis using estimates of variances and effect sizes derived from similar studies (Tamborlane, 2005; Schwartz et al., 2005; McGillis-Bindler \& Bruya, 2005) indicated that a sample size of 45 was sufficient to detect differences of a medium effect size ( $d=.5)$ (Cohen, 1988) between finger prick blood parameters (blood glucose, $\mathrm{HbAlc}$ and TC) and venipuncture technique for the same variables to yield $90 \%$ power with significance level of .05 for a two-sided paired samples t-test. However, 60 students were recruited, six from each of the ten schools to allow for attrition.

McGillis-Bindler \& Bruya, (2005), identified children at risk for overweight, cardiovascular diseases, and type 2 diabetes, compared finger prick capillary whole blood to the "gold standard" of fasting serum values for glucose and lipoprotein levels. Correlations were found only for HDL-C $(\mathrm{r}=.591, \mathrm{p}=.003)$ and triglycerides $(\mathrm{r}=.534$, 
$\mathrm{p}=.010$ ) when comparisons were made between the non-fasting finger prick method and fasting venipuncture method. They selected 23 subjects from a sample of 111 to give $80 \%$ power for a one tailed test to detect a difference of $5 \%$ in the correlation analyses (McGillis-Bindler \& Bruya et al., 2005).

Tamborlane et al. (2004) compared finger prick HbA1c levels of 200 type 1 diabetic children aged 7-17 years assayed by a Point-of Care (POC) instrument, the DCA 2000 , with the DCCT/EDIC central laboratory assay and found a strong correlation between the DCA and the DCCT/EDIC $(r=0.94, p<0.001)$. However, the DCA HbA1c levels were significantly higher than the DCCT/EDIC central laboratory values with a mean difference of $0.2 \%, \mathrm{p}<.001$ (95\% $\mathrm{CI}$ of 0.14 to 0.23$)$.

\section{Procedure}

The study was conducted with the approval of the Institutional Review Board, Florida International University, and the Jamaican Ministry of Education, Youth, and Culture, and the Ministry of Health and Environmental Control (Appendices A-C). The principals in the selected schools were contacted for their school's consent to participate and to gain access to selecting students (Appendix D). The researcher visited each of the ten randomly selected schools and obtained written permission from the principals. On this visit, a Contact Person (CP) was selected to include the school nurse, guidance counselor or the senior nutrition teacher. Written consent of the CP was obtained on that visit (Appendix E). The selected CP in each school was briefed on the study protocol. After obtaining permission from the principals and CPs, the researcher began to recruit students from each grade level. Students were stratified by grade level and gender 
and five names randomly selected per grade level. The researcher explained the study to the student and asked them to take the combined consent/assent forms (Appendix A) home for their parent's signature. Students whose parents have consented were asked to sign the assent form (Appendix A) after the study was explained to them. Contact persons were responsible for organizing the students on data collection days and called the students the night before to remind them of the overnight fast. The demographic and socioeconomic questionnaire (Appendix F) was self-administered on data collection day and questionnaires returned to the researcher on the same day.

Finger prick blood collection was performed by workers of the Mobile Unit of the Heart Foundation of Jamaica (Appendix G), and the Diabetes Association of Jamaica (Appendix H). Venous blood draws were taken by the phlebotomist of the Central Medical Laboratory, Kingston (Appendix I). Anthropometric measures, BP, and physical examination for Acanthosis Nigricans were obtained by the school nurse and the researcher. The entire data collection lasted for three weeks by targeting one school per day.

Workers of the Heart Foundation of Jamaica performed the finger prick tests for TC and FBG. Students were pricked a second time by a worker from the Diabetes Association of Jamaica who conducted the finger prick tests for HbAlc only on all 276 subjects. The phlebotomist of the Central Medical Laboratory accompanied the research team to collect venous blood samples and test for HbA1c, TC, and FPG. Venous blood samples were stored and taken back to the laboratory in Kingston for testing on the same day. All 60 samples were tested at the same laboratory using standard laboratory 
procedures for testing TC (chemistry dry slide technology), FPG (hexokinase method), and HbA1c (boronate- affinity HPLC method).

Students were instructed to complete a 12 hour fast before the blood collection. On each test day students reported to the "sick bay" where they were interviewed to determine if they had fasted the night before. Those students who were not fasting were not used in the study. Weights, heights, waist, and hip circumference were measured by the researcher and the CP. Six randomly pre-selected students from each school had their blood samples taken by the phlebotomist of the Central Medical Laboratory using venipuncture. The phlebotomist prepared the blood for testing and transported it to the laboratory where analysis was performed within 24 hours.

After completion of dietary intakes, physical activity questionnaire, anthropometric measures, examination for $\mathrm{AN}$, demographic data and two measures of blood parameters, each subject was given a monetary incentive of US\$5.00 along with other tokens of appreciation. Tokens included donations of daily planners, notebooks, pens, pencils, pocket-sized hand lotion, toothpaste, rubber stress balls, T-shirts, and sunvisors. Those who participated in the comparison study were given an additional US $\$ 5.00$ after completing the venipuncture. Students were served a continental breakfast of fruit juice/fruit plate, choice of sandwiches, danish/muffins, after blood collection to prevent possible hypoglycemic episodes (Appendix J for Study Protocol). 
All instruments were pilot tested in a high school that was not part of the sample schools but similar to the study schools. Informed consent (Appendices KI-III) was obtained for pilot testing of instruments. Instruments for measuring AN (Appendix L), and PA (Appendix M) have been used and reliability determined in previous studies (Burke et al, 1999; Crocker, Bailey, \& Faulkner et al., 1997). In one study comparing physical activities of 84 children ages 9-15 years using the Physical Activity Questionnaire for Children (PAQ-C), Crocker, et al (1997) found acceptable levels of test-re-test reliability for both males $(r=.75)$ and females $(r=.82)$. The scale developed by Burke et al., (1999) for determining locations and severity of AN has been successfully used in other studies among Mexican Americans. The scale had good replicability $(k>.68)$ for measuring the severity and location of AN on the neck region (Burke et al., 1999). The demographic questionnaire (Appendix F) and food recall (Appendix N) were developed by the researcher and were tested and validated before being administered. These instruments were tested among a similar sample of 40 students from a high school not included in the study.

\section{Data Collection and Description of Instruments}

Demographic, Anthropometric, Medical Data, Physical Activity and Dietary Intakes

Specific demographic and anthropometric data from subjects were collected at each selected school. Demographic data included gender, age, race/ethnicity, parents' occupation, place of residence, parent's income and educational level, family history of diabetes, obesity, and cardiovascular disease (Appendix F). 
Anthropometric

Anthropometric data such as weight, height, waist and hip circumferences were collected and recorded by the researcher and $\mathrm{CP}$ for each subject at each selected school (Appendix O). Students were weighed using the Professional Medical Beam Balance scale (Health-O-Meter, model 402EXP) with weight capacity of $220 \mathrm{~kg}$ and $450 \mathrm{lbs}$ and weight graduations of 100 grams and 4 ounces respectively. Weights were measured in $\mathrm{kg}$. The height rod with increments in $\mathrm{cm}$ as well as inches with height range of 24 " to 84 " in $1 / 8$ " increments and $60 \mathrm{~cm}$ to $213 \mathrm{~cm}$ in $1 \mathrm{~mm}$ increments was used. Subjects were weighed in their school uniforms and were asked to remove their shoes before weighing. Weights were recorded to the nearest $0.1 \mathrm{~kg}$. The scale was disinfected and recalibrated after each use and the process repeated. Heights were taken using a stadiometer and recorded in centimeters. Subjects were asked to stand straight with weight evenly distributed on both feet, feet together with arms at the sides. Subjects were asked to look straight ahead. The headpiece was lowered to touch the head and lightly compress the hair. Heights were measured to the nearest $0.5 \mathrm{~cm}$.

BMI was calculated as weight $(\mathrm{kg})$ divided by height $\left(\mathrm{m}^{2}\right)$. Although BMI cut-off points vary among different ethnic groups for the same level of body fat, research has shown that an increase in T2DM and CVDs is linked to increase BMI (Singh, et al., 2004). Research indicated that $85 \%$ of children with T2DM were overweight (BMI > $85^{\text {th }}$ percentile) or obese $\left(\mathrm{BMI}>95^{\text {th }}\right.$ percentile). Body mass index as determined by the International Obesity Task Force (IOTF) for the following categories were used to classify subjects as normal weight $(18.5 \leq \mathrm{BMI} \leq 24.9)$, overweight $(25.0 \leq \mathrm{BMI} \leq 29.9)$, and obese $(\mathrm{BMI} \geq 30)$. Among the obese category, subjects were further classified as 
class I (BMI $\geq 30.0-34.9)$, class II $(\mathrm{BMI} \geq 35-39.9)$ and Class III $(\mathrm{BMI}>40)$ obese. The data collected in this section was used primarily to describe the study population as well as factored in the logistic regression analyses. BMI was coded as follows for descriptive purposes;

$$
\begin{array}{ll}
\circ \quad 1=\text { Underweight }(\mathrm{BMI} \leq 18.5) \\
\circ \quad 2=\text { Normal Weight }(18.5 \leq \mathrm{BMI} \leq 24.9) \\
\circ \quad 3=\text { Overweight }(25.0 \leq \mathrm{BMI} \leq 29.9) \\
\circ \quad 4=\text { Class I Obesity (BMI } \geq 30-34.9) \\
\circ \quad 5=\text { Class II Obesity (BMI } \geq 35.0-39.9) \\
\circ \quad 6=\text { Class III Obesity }(\mathrm{BMI} \geq 40.0)
\end{array}
$$

For the logistic regression, $\mathrm{BMI}$ was coded as $1=\operatorname{risk}(\mathrm{BMI} \geq 25)$, and $0=$ no risk $(\mathrm{BMI}<25)$. Actual BMI of study subjects and self-reported BMI equivalents from silhouettes (Appendix P) were used for correlating BMI with family history of overweight and obesity.

Family history of obesity based on BMI percentages was measured using a scale of 1-9 silhouettes for both males and females. The scale of 1-9 corresponded to BMI percentages (Appendix P) for equivalent BMI. A scale of 5 and above represented overweight and obesity. This scale was developed by Bulik, Wade, Heath et al. (2001) and was successfully used to determine obesity among 16,728 female and 11,366 male Caucasians ages 18-100 years. Results using receiver operating curves (ROC) indicated that these figures effectively classified individuals as obese or thin (Bulik et al., 2001). A reliability check for self-reported BMI data using scale and compared to actual BMI 
measures of 181 women and 160 men revealed high correlations between actual BMI and self-reported BMI for men $r=.90$ and women $r=.94$ (Bulik et al., 2001).

The scale was pilot tested on a group of 37 Jamaican adolescents ages $14-19$ years in one high school. Pilot study results found significant correlations between actual BMI for subjects and perceived BMI using the silhouettes $(r=.45, \mathrm{p}<.05)$. The scale was also used to identify family history of obesity by having students circle the silhouette they thought most closely resembled their mother and father. Results of the pilot study indicated strong correlation between the subjects perceived BMI (from silhouettes) and subject's perceived BMI of their father $(r=.46, p=.008)$. No correlation was found between subject's actual BMI and their perceived BMI of their father. However, significance was found for subject's actual BMI and their perception of their mother's BMI $(\mathrm{r}=.40, \mathrm{p}<.01)$. Subjects' mean BMI for perceived and actual BMI were $24.1 \mathrm{~kg} /$ $\mathrm{m}^{2}$ and $23.7 \mathrm{~kg} / \mathrm{m}^{2}$ respectively. Actual BMI measurements of study subjects classified 11 or $29 \%$ of the sample as overweight (BMI $\geq 25 \mathrm{~kg} / \mathrm{m}^{2}$ ) compared to 12 or $32 \%$ for perceived (self-reported) BMI. Based on pilot tests results the silhouettes were included in the main study. Family history of overweight/obesity was determined as BMI $\geq 25$ $\mathrm{kg} / \mathrm{m}^{2}$ and $\mathrm{BMI} \geq 30 \mathrm{~kg} / \mathrm{m}^{2}$ if one parent was overweight or obese respectively. In the present study data were coded as $0=$ no family history of overweight/obesity and $1=$ family history of overweight/obesity.

Measuring Central Adiposity

Excess abdominal fat is more resistant to the action of insulin which in turn increases the risk for CVDs, T2DM and hypertension. In a study of Asian Indians, up to 
$20 \%$ of subjects although not overweight, had central obesity putting them at risk for CVDs, T2DM and hypertension (Gopalan, 1998). Central adiposity poses a risk for diabetes and insulin resistance. Cut-off values of waist circumference (WC) of $85 \mathrm{~cm}$ and $80 \mathrm{~cm}$ with WHR of 0.89 and 0.81 for men and women respectively are recommended (Ramachandran, 2004).

In this study, both waist circumference and BMI were used to determine those at risk for T2DM and CVDS. Waist circumference of $\geq 94 \mathrm{~cm}$ and $\geq 80 \mathrm{~cm}$ shows increased risk of obesity-associated metabolic complications for adult Caucasian males and females, $\geq 102 \mathrm{~cm}$ and $\geq 88 \mathrm{~cm}$ shows substantial risks for both males and females respectively (Ramachandran, 2004).

Waist circumference was measured by placing a non-stretchable tape around the waist at the region of the navel for males and between the bottom of the rib and top of the hip bone for females. Subjects were asked to stand erect with abdomen relaxed, arms at the side and feet together. The participants were asked to breathe normally then the measurements were taken at the end of the normal expiration. Measurements were recorded to the nearest $0.1 \mathrm{~cm}$. Waist circumference was coded as a categorical variable:

$\circ 0=$ normal $(<94 \mathrm{~cm}$ and $<80$ for males and females $)$

○ $\quad 1=\geq 94 \mathrm{~cm}$ and $\geq 80 \mathrm{~cm}$ for adult males and females), increased risk for T2DM and CVDs.

- $2=\geq 102 \mathrm{~cm}$ and $\geq 88 \mathrm{~cm}$ for males and females), substantial risk for T2DM and CVDs 
Waist circumference was classified as $1=$ risk for $\mathrm{WC} \geq 94 \mathrm{~cm}$ and $\geq 80 \mathrm{~cm}$ for males and females, and $\geq 102 \mathrm{~cm}$ and $\geq 88 \mathrm{~cm}$, and $0=$ no risk for $\mathrm{WC}<94 \mathrm{~cm}$ and $<80$ for males and females.

Hip measurements were taken at the tip of the hip bone in males and at the widest point of the hip and buttocks of the females. Measurements were recorded to the nearest 0.1 centimeter. To arrive at the waist- to-hip ratio, the waist circumference was divided by the hip circumference. A healthy waist-to-hip ratio for female is 0.8 and below and for males 1.0 or below (Dalton, Cameron \& Shaw et al., 2003). People with more weight around the waist face more health risks than those who carry the weight around the hip (Dalton et al., 2003). Waist-to-hip ratio was coded as:

$$
\begin{aligned}
& \circ 0=\text { healthy WHR }<0.8 \text { for females, and }<1.0 \text { for males. } \\
& \circ \quad 1=\text { increased risk for CVDs }>0.85 \text { for females and }>1.0 \text { for males. }
\end{aligned}
$$

Waist circumference and WHR have been found to be associated with both CVDs and T2DM in adults. However, data on adolescents are needed. In one study of 11,247 Australians aged $>25$ years examined for cardiovascular disease risks by using waist circumference, waist-hip ratio and BMI, WHR had the strongest relationship with T2DM, dyslipidemia (women only) and hypertension. Strong associations were found for BMI and hypertension in women and for T2DM in men. WHR had a strong relationship with CVDs risk factors before adjustment for age (Dalton et al., 2003).

In another study of 313 men and 382 women in Canada, waist and hip circumferences were also found to have effects on cardiovascular disease risk factors. Both men and women with large waist circumference had significantly lower HDL cholesterol concentrations $(\mathrm{p}<.05)$ and high fasting triacylglycerol, insulin and glucose 
concentrations $(\mathrm{p}<.01)$. A large waist circumference was associated with high LDL-C and BP for women only and with low HDL and high glucose in men $(\mathrm{p}<.05)$. Both high triacylglycerol and insulin concentrations were found in men and women $(\mathrm{p}<.05)$ (Seidell, Perusse, Jean-Peirre et al., 2001). Waist circumference and WHR measurements are also significant for T2DM patients since T2DM is largely associated with obesity. Studies have shown that patients with T2DM have higher WHR than non-diabetics (Seidell, Han, Feskens, et al., 1997).

Blood Analysis

Capillary blood samples were tested by the workers from the Heart Foundation of Jamaica for glucose and total cholesterol. HbAlc samples were tested by a Diabetes Association of Jamaica worker. Students reported to the "sick-bay" in each school, were interviewed and then tested. Sixty randomly selected students from the larger study population had their blood drawn by venipuncture by the phlebotomist of the Central Medical Laboratory. Venous blood samples were prepared and transported to the laboratory in Kingston for analysis.

\section{Blood Glucose}

In this study both fasting blood glucose (FBG) and fasting plasma glucose (FPG) were measured on the same day. Fasting blood glucose was measured using the Accu Chek Advantage Blood Glucose Monitor (Roche Diagnostics of New Zealand) for the finger prick method. With this procedure, blood glucose concentrations were measured with a small amount $(4 \mu \mathrm{L})$ of capillary whole blood taken from the fingertip. Capillary 
blood was obtained by using a sterile lancet to prick the finger. The blood was applied directly to the reagent strip. Successive drops of blood were taken for measuring TC at that moment. One drop of capillary blood was applied to the test strip and placed in the glucose monitor. The instrument uses biosensors to generate a current. The size of the current indicates the amount of glucose present in the blood. Test time for the FBG was about 15 seconds and results were available immediately. The results were entered on the data sheet (Appendix O) immediately.

Validity and accuracy of Accu Chek Advantage Blood Glucose Monitor (Roche Diagnostics of New Zealand) has been determined in a number of studies. In a study which evaluated the variability of results between point-of-care (POC) testing glucose meters using fresh whole blood and the central laboratory analyzer, the Accu Chek group of glucose monitors showed the lowest bias but there were considerable differences between laboratory methods and the other glucose meters (Khan, Vasquez, Gray, et al., 2006). Successive glucose meter readings agreed well when testing hypoglycemia, but readings with differences greater than $10 \%$ occurred more than $61 \%$ of the time in the hyperglycemic values and $20 \%$ occurred $57 \%$ of the time in the hypoglycemic range using the Accu Chek Advantage when compared to the central laboratory values (Khan, et al., 2006).

Accuracy and precision of this instrument was also tested in an Australian study using capillary blood samples from 49 diabetic patients attending a clinic (Cohen, Boyle, Delaney et al., 2005). Blood samples were evaluated using five glucose meters and compared with a reference; the YSI Glucose Analyzer (Yellow Springs Instruments, Ohio, USA). Coefficient of variation was calculated to determine precision. Coefficient 
of variation $(\mathrm{CV})$ ranged from 2.8 to $5.5 \%$ and were close to the values given by the manufacturers, except for the Accu Chek Advantage (Roche). Coefficient of variation for the Accu Chek Advantage ranged from 3.64 to $2.62 \%$ in this study compared to the manufacturers listed variability of $<3.1 \%$. Of the five glucose meters tested, only one (CareSens) from Australian met the ADA (Australian Dietetic Association)

recommendation of $<5 \%$ bias (deviation from reference). In this study, bias ranged from 4.0 to $15.5 \%$ (Cohen, et al., 2005). Most meter readings were higher than the reference device with the Accu Chek being $0.6 \mathrm{mmol} / \mathrm{L}$ higher than the reference (Cohen, et al., 2005).

Since blood glucose varies throughout the day in response to meals and exercise, in this study measures were taken after a 12 -hour fast and first thing as the students arrived at school. Both finger prick and venipuncture were carried out at the same time to reduce variations in glucose concentrations. Whole blood from capillary was used for the finger prick method and plasma for the venipuncture.

For the venipuncture, the phlebotomist accompanied the researcher to each school and collected these samples before anthropometric measures and other data were collected. To prevent glucose variability due to glycolysis, the sample was treated with a glycolysis inhibitor sodium fluoride (2.5 $\mathrm{mg}$ fluoride/mL of blood) to slow glycolysis. This process may take up to 2 hours to stop glycolysis. If the sample is not tested early the blood samples can show a 5-7\% decrease in glucose values (Nichols, 2006). After treating with a glycolysis inhibitor, samples were set on ice for transportation to the laboratory. Glucose concentrations in whole blood usually become stable after four hours and can be kept for 72 hours at room temperature if an inhibitor such as fluoride is 
used (Chan, Swaminathan, \& Cockran, 1989). Four hours were sufficient time to transport the samples from each school back to the laboratory in Kingston for testing.

For the venous method, a small sample of blood $(3 \mathrm{~mL})$ was obtained from the basilic and cephalic veins at the antecubital fossa. that is, the area inside the bend of the elbow where the veins are very superficial and close to the skin. Analysis of venous blood was done using the COBAS MIRA Plus Analyzer (Roche Diagnostics) using the Glucose Hexokinase Liquid Reagent (Roche Diagnostics). The principle is based on a modification of Slein (1963) enzymatic testing using hexokinase and glucose-6phosphate-dehydrogenase to catalyze the reaction. Results were referenced to the FDA method for measuring blood glucose.

Differences have been noted between whole blood and plasma glucose levels. Whole blood levels are 12 to $15 \%$ lower than plasma glucose because of less water content in RBCs compared to plasma (Nichols, 2006). After a fast, venous blood is approximately 2 to $5 \mathrm{mg} / \mathrm{dL}$ lower than capillary glucose due to glucose absorption and cellular metabolism. Capillary glucose can be 20 to $70 \mathrm{mg} / \mathrm{dL}$ higher than venous blood samples after a meal because of post-prandial effects of insulin on cellular uptake and metabolism ((Nichols, 2006). Therefore all blood samples were taken after an overnight fast of 12 hours.

Fasting plasma glucose (FPG) was classified based on the most current ADA criteria (ADA, 2005). Blood glucose levels included continuous as well as categorical values. Categorical values were coded as 0-2 to represent the following cut-off points. However, categories 1 and 2 were combined and treated as at risk for T2DM. 


$$
\begin{aligned}
& \circ \quad=\text { Normal (fasting glucose }=\mathrm{FPG}<100 \mathrm{mg} / \mathrm{dL} \\
& \circ \quad 1=\text { Impaired fasting glucose }(\mathrm{IFG})(\mathrm{FPG} \geq 100 \mathrm{mg} / \mathrm{dL} \text { and }<126 \mathrm{mg} / \mathrm{dl}) \\
& \circ \quad 2=\text { Diabetes }(\mathrm{FPG} \geq 126 \mathrm{mg} / \mathrm{dLl}) .
\end{aligned}
$$

The ADA recommends three criteria for diagnosis of diabetes mellitus which include 1) checking for symptoms such as polyuria, polydipsia, unexplained weight loss, and a causal plasma glucose concentration $>200 \mathrm{mg} / \mathrm{dL}, 2$ ) fasting plasma glucose $>126$ $\mathrm{mg} / \mathrm{dL}$, and 3) two-hour postload glucose $>200 \mathrm{mg} / \mathrm{dL}$ performed during an oral glucose tolerance test (OGTT) using a glucose load of $75 \mathrm{~g}$ of anhydrous glucose dissolved in water (James, 2006). Reference intervals for glucose concentrations in healthy children range from 3.3-5.6 mmol/L (60-100mg/dL) and for adults it is $4.1-5.9 \mathrm{mmol} / \mathrm{L}(74-106$ $\mathrm{mg} / \mathrm{dL}$ ) (Sacks, 1999). However, the ADA criteria was used in this study for diagnosing diabetes rather than these reference intervals

Total Cholesterol (TC)

Capillary testing of total cholesterol was carried out by the workers of the Heart Foundation of Jamaica using the Accutrend GCT Cholesterol Monitor (Roche, Diagnostics, Mannheim, Germany). Measurement of TC in this study used enzymatic methods (cholesterol esterase). Cholesterol esters are cleaved by the cholesterol esterase to form free fatty acids and cholesterol. The products are further oxidized by cholesterol oxidase in the presence of peroxidase to form a red substance called quinoneamine. The instrument measured the intensity of the reaction color using "reflectance photometry" and then calculated TC using a lot specific algorithm. The result was displayed in $\mathrm{mg} / \mathrm{dL}$ or $\mathrm{mmol} / \mathrm{L}$. The instrument measured a range of TC of $150-300 \mathrm{mg} / \mathrm{dL}(3.88-7-76$ 
$\mathrm{mmol} / \mathrm{L}$ ). Manufacturer's precision of the instrument for measuring TC is stated as $<5 \%$ and for accuracy $\pm 5 \%$. Results are available in 3 minutes and uses only one drop of blood for testing.

Research is lacking on the reliability of the Accutrend GCT in the testing of total cholesterol. However, reliability of the Accutrend GCT was tested for TG and showed that coefficient variations for different capillary TG concentrations were $3.3 \%$ for high TG and 5.3\% for low TG (Moses, Calvert, \& Storlien, 1996). When compared to plasma TG concentrations, the Accutrend GCT capillary measures of TG had a strong correlation $(r=0.94)$ (Moses et al., 1996).

Point-of-care tests are to be validated for accuracy and precision. Accuracy refers to the agreement between the monitor and standardized laboratory test on the same parameters, whereas, precision refers to the reproducibility of these tests (Taylor \& Lopez, 2004). Guidelines for accuracy and precision of these instruments are determined by the NCEP and CDC. The NCEP recommends a coefficient of variation between laboratory testing and the monitor to be $\leq 3 \%$. Average bias (precision) according to the CDC should not be greater than $\pm 3 \%$.

Both venous and capillary blood samples were tested and compared in the present study. Earlier research indicated high positive bias for fingerstick plasma capillary testing of TC versus venous serum testing. In one study (Greenland et al., 1990) cholesterol concentrations in fingerstick derived plasma were consistently higher and gave a positive bias of $3.6 \%$ which means this method alone would classify subjects at risk when they are not. Cutoff points for cholesterol by the National Cholesterol Education Program (NCEP) refer to venous serum samples. 
Cholesterol guidelines for children as set by the National Cholesterol Education

Project, sponsored by the National Heart, Lung and Blood Institute, suggested a desirable blood lipid profile for children. Cholesterol level less than $175 \mathrm{mg} / \mathrm{dL}$ was recommended for children. Compared to adults, the ATPIII panel suggested $\mathrm{TC}<200 \mathrm{mg} / \mathrm{dL}$ as desirable, $200 \mathrm{mg} / \mathrm{dL}$ as borderline high, and $\geq 240 \mathrm{mg} / \mathrm{dL}$ as high. Total cholesterol was coded as $0-2$ to represent the following categories;

$$
\begin{aligned}
& 0=\text { Normal }(\mathrm{TC}<170 \mathrm{mg} / \mathrm{dL}) \\
& 1=\text { Borderline }(\mathrm{TC} 170-200 \mathrm{mg} / \mathrm{dL}) \\
& 2=\text { Abnormal }(\mathrm{TC}>200 \mathrm{mg} / \mathrm{dL})
\end{aligned}
$$

Total cholesterol was further coded as $1=$ risk $(\mathrm{TC}>170 \mathrm{mg} / \mathrm{dL})$ and $0=$ no risk $(\mathrm{TC}<$ $170 \mathrm{mg} / \mathrm{dL}$ ) for statistical analyses.

\section{Methodology for Testing Total Cholesterol}

A small drop of blood which was applied directly to the testing strip in the Accutrend GCT Cholesterol Monitor analyzer, immediately after the finger was pricked for the FBG blood sample. The technology used for testing TC with point-of-care testing monitors involves the use of a cholesterol esterase which hydrolyzes cholesterol esters (Taylor \& Lopez, 2004). Hydrogen peroxide is produced when cholesterol oxidazes oxidize the cholesterol. Readings are made due to color differences produced when the hydrogen peroxide further reacts with a substrate in the presence of peroxidase. A light is reflected off the test strips due to the color change. The monitor converts the reading into a cholesterol level and displays the reading. It is the height of the color peak which indicates the amount of hydrogen peroxide and consequently the amount of cholesterol 
from the sample (Taylor \& Lopez, 2004). The venous sample was taken by the phlebotomist of the Central Medical Laboratory on the same day and taken back to Kingston for analysis using standard laboratory procedure. Venous samples were tested using the VITROS 350 analyzer which used a chemistry dry slide technology for measuring lipid profiles.

\section{Glycated Hemoglobin}

In this study $\mathrm{HbAlc}$ was measured by both finger prick and venous blood samples. Comparisons were made to determine the variability of these methods and to determine if there were significant differences between $\mathrm{HbAlc}$ as measured by both methods. Finger prick method is cheaper than laboratory testing especially among people with no health insurance as expected in the Jamaican population.

In a comparison testing $\mathrm{HbA} 1 \mathrm{c}$ was measured in the same day visit with two laboratory methods, HbAlc values were consistently lower for the capillary blood samples. The aim of the study was to determine if same-day-visit testing of HbAlc (using capillary blood finger prick samples) could be used in these health care centers for more efficient patient care and improved diabetes management (Schwartz et al., 2005).

The normal $\mathrm{HbA} 1 \mathrm{c}$ range for a healthy person is $4-5.9 \%$. The International Diabetes Federation and American College of Endocrinology recommends that $\mathrm{HbAlc}$ values below 6.5\% whereas the American Diabetes Association extends this range to $7 \%$. Ranges above 7\% represent poor glucose control (Nakamota, 2006). In this study, the International Diabetes Federation guidelines of $<6.5 \%$ will be used as the cut-off point 
for normal levels of $\mathrm{HbAlc}$. For statistical analyses $\mathrm{HbAlc}$ measures were coded as dichotomous values to represent the following;

$$
\begin{aligned}
& \circ \quad 0=\leq 6.5 \% \text { for normal } \\
& \circ \quad 1=>6.5 \% \text { poor glucose control. }
\end{aligned}
$$

The present study assessed $\mathrm{HbAlc}$, the standard method of assessing long-term glycemic control which gives the glycemic history of the subject over the past three months. Measurement of $\mathrm{HbAlc}$ is needed in diabetic control since glucose irreversibly binds or glycates with red blood cell hemoglobin, therefore more glucose equates to more binding. Current guidelines recommend $\mathrm{HbA} 1 \mathrm{c}$ values below 7.0. HbAlc values increasing from 5.0 to 7.0 in non-diabetics, have been associated with increased risks of CVDs (Shankar, Klein, \& Moss, 2007). The International Federation for Diabetes and the World Health Organization have not agreed to the use of HbAlc only in the diagnosis of T2DM, however studies show that when used with other measures such as FPG and BMI, HbAlc is effective for screening (Norberg, Eriksson, Lindahl et al., 2006).

In this study HbAlc was measured using the Nycocard manufactured by AxisShield. The blood was drawn into a short micro-pipette by finger prick and dropped into a pre-dispensed test tube where it was mixed with blue borate buffer reagent and left for three minutes. The machine is equipped with a timer to indicate when to pick up the tube and give it a spin. After spinning, 25 microliters of the mixed solution were drawn with an automatic pipette. This sample was then expelled into a mixing well where it was absorbed by a filter paper membrane. The filter soaked up the reagent within seconds, the pipette was changed, and 25 microL of wash solution delivered to the mixing well. 
After this was absorbed, the Nycocard pen read the HbAlc and displayed the result on the LCD screen (Nycocard Diabetes Screening).

The Ncyocard is Diabetes Control and Complications Trial (DCCT) calibrated and used up to 5 microliters of blood from finger prick or venipuncture which made it ideal for use with children and adolescents. The results were available within four minutes and measures were reported as one standardized $\mathrm{HbA} 1 \mathrm{c}$ value. Measurements for $\mathrm{HbA} 1 \mathrm{c}$ using fasting venous samples were carried out using standardized laboratory procedure (boronate affinity HPLC) which is DCCT corrected.

Measurement of venous $\mathrm{HbA1c}$ samples was carried out using the PDQ Plus Analyzer from Primus Corporation which is certified by National Glycohemoglobin Standardization Program, USA. This equipment used high-performance boronate affinity chromatography to separate glycated hemoglobin. The hemoglobin fractions were then monitored by light absorption at 413 nano meters $(\mathrm{nm})$. Results were available within two minutes.

Blood Pressure (BP)

Research indicated that up to $17-32 \%$ of adolescents with T2DM have hypertension (Fagot-Campagna, Pettitt, \& Engelgau, 2000). According to a Pan American Health Organization (PAHO) 2008 Report, hypertension is the number one risk factor for death in the region. In another study of Pima Indians ages 5-19 years, $85 \%$ were obese, $14 \%$ had hypertension and $30 \%$ had dyslipidemia (Fagot-Campagna et al., 2000). 
The researcher measured blood pressure using a sphygmomanometer. Students were allowed to rest for five minutes before the measurements were taken. An average of two readings was used in the analysis. Blood pressure exceeding the $95^{\text {th }}$ percentile for height, age and sex were used to define the diagnosis of hypertension. The height percentile, age and sex were used to ascertain the $90^{\text {th }}$ and $95^{\text {th }}$ percentiles for BP (Appendices Q and R). Adolescents who plotted below the $90^{\text {th }}$ percentile for height, age, and gender were considered to have normal BP. Adolescents who plotted between $90-95^{\text {th }}$ percentile were classified as having "pre-hypertension" which means they are at risk to developing hypertension. Subjects whose systolic or diastolic BP plotted higher than the $95^{\text {th }}$ percentile for age, gender, and height measurements were classified as hypertensive and referred to follow-up. For 17-19 year olds, the $95^{\text {th }}$ percentile which is close to $140 / 90 \mathrm{~mm} \mathrm{Hg}$, the cut-off point for adults, was used to classify subjects as hypertensive. The American Academy of Pediatrics (AAP), American Heart Association (AHA), and the American Medical Association (AMA) recommended routine screening of adolescents and children during preventive visits since up to $28 \%$ of children have secondary hypertension for which early detection may be beneficial. Blood pressure was coded as 0-2 to represent the following;

$\circ \quad 0=$ Normal $\left(\mathrm{BP}<90^{\text {th }}\right.$ percentile for height, age and gender $)$

○ 1 = pre-hypertensive, (BP between the $90-95^{\text {th }}$ percentile for height age, and gender)

○ 2 = hypertensive (BP higher than the $95^{\text {th }}$ percentile for age, gender and height). 
Pre-hypertensive and hypertensive measures were further collapsed into two categories wherein $1=$ risk $\left(\mathrm{BP}>90^{\text {th }}\right.$ percentile $)$ and $0=$ no risk $\left(\mathrm{BP}<90^{\text {th }}\right.$ percentile $)$ for analyses purposes.

\section{Acanthosis Nigricans (AN)}

The researcher examined each subject for the presence of Acanthosis Nigricans (AN), a dark, raised skin in areas such as the axilla, neck, knuckles, and knees which is believed to result from hyperinsulinemia. Acanthosis Nigricans is manifested by a darkening of the skin, velvety plaques of body folds and is most commonly seen around the neckline or in the axilla in children who have greater body weight and fat mass, higher serum insulin levels and lower insulin sensitivity (McGillis-Bindler\& Bruya, 2005, Burke et al., 1999). Examination of AN was significant to the study since AN is becoming more prevalent among obese patients and those with type 2 diabetes (Burke et al., 1999). Subjects with AN were also at risk for atherosclerotic cardiovascular diseases (Katz \& Feldman, 2006).

The presence of $\mathrm{AN}$ is a marker for the risk factor of hyperinsulinemia. Hyperinsulinemia leads to binding of insulin-like growth factor (IGF) receptors on keratinocytes and fibroblasts and ultimately hyperplasia of the skin (Cruz, 1992). Acanthosis Nigricans is associated with hyperinsulinemia, which is a consequence of insulin resistance often associated with obesity (Burke et al., 1999; McGillis-Bindler \& Bruya, 2005). Acanthosis Nigricans has been reported in $60-90 \%$ of young people with T2DM (Scott, et al., 1997) and is a physical marker for insulin resistance and appears to be more prevalent in some ethnic groups (Tajima, 2002; Stuart, et al., 1998). 
In this study a quantitative scale (Appendix K) which measured the severity as well as locations for AN was used. The scale includes six AN measures; neck, axilla, neck texture, knuckles, elbows and knees to determine the presence or absence of $\mathrm{AN}$ as well as the severity (Appendix L). However, it was not convenient to measure AN on the axilla in the current study. A quantitative scale ranging from 0-4 was used to determine the severity of $\mathrm{AN}$ on the neck wherein $0=$ absent, $1=$ present, $2=$ mild, $3=$ moderate and $4=$ severe. For the neck texture a scale of $0-3$ wherein $0=$ smooth to the touch, $1=$ rough to touch, 2 = coarse with raised skin, and $3=$ extremely coarse and clearly visible. For the knuckles, elbows and knees, a qualitative scale using the terms "present or absent" was used (Appendix L). Acanthosis Nigricans easily resembles psoriasis but can be differentiated because unlike psoriasis, the plaques of $\mathrm{AN}$ do not present with inflammation. For the regression analyses, presence of AN only on the neck was used and coded as present $=1$, and absent $=0$. Descriptive statistics such as means and frequencies were used to determine the severity and other locations of AN.

Burke et al. (1999) in a study of 406 participants examined for AN using the same scale as in this study. The neck, axilla, elbows, knuckles and knees were examined by two independent observers. Results showed high inter-observer concordance and $\mathrm{k}$ statistics of the scale. The scale was found to have high inter-observer reliability $(\mathrm{k}>.68)$ and correlated well with insulin resistance (IR) and BMI among Mexican Americans (Burke, et. al., 2001). Only the neck region had high $\mathrm{k}$ statistics and the researchers excluded the other areas from their analyses. A k statistic that is $>.75$ is considered excellent reproducibility and $.4<\mathrm{k}<.75$ represents good reproducibility (Burke et al., 2001). In our study, data were collapsed for analysis in the logistic regressions using 
presence (1) or absence (0) of AN for the neck only. However, descriptive statistics were also used to determine the severity of AN in this population.

Physical Activity (PA)

Research has shown that children need up to 60 minutes of exercise per day (Klein et al., 2004). Physical activity has been shown to increase insulin sensitivity in children (Schmitz, Jacobs, Hong, et al., 2002). This increased insulin sensitivity is seen among obese children undergoing regular exercise then it decreases when they revert to a sedentary life style (Schmitz, et al., 2002). Obesity and dietary factors have been shown to affect the metabolic response to exercise, for example, high- fat feeding reduces the growth hormone response to exercise (Cappon, Ipp, Brasel, et al., 1993). Earlier studies indicated that a change to a sedentary lifestyle similar to the effects of Westernization on Australian Aborigines resulted in increased life-style-related chronic diseases such as T2DM, premature CVDc, dyslipidemia, hypertension and hyperinsulinemia (O’Dea, White, \& Sinclair, 1998), therefore, low physical activity was considered a risk factor for T2DM and CVDs.

In our study, physical activity was measured by using the Physical Activity Questionnaire for Older Children (PAQ-C). The PAQ-C is a self-administered 7-day recall measure which assessed general physical activity levels during the school year (Appendix M). The instrument consisted of 9 questions that assessed activity levels at different times of the day including school and out of school activities. Each question has five responses and was scored from 1-5 where $1=$ inactive (student does not participate in that particular activity at all), $2=$ low activity level (activity is performed 1-2 times in 
the past seven days), $3=$ moderately active (activity is performed 3-4 times in the past seven days, $4=$ active (activity is performed 5-6 times in the past seven days, $5=$ very active (activity is performed $>7$ times in the past seven days). An average of all 9 items was taken to determine the activity level of the child.

With this method, each item has an equal weight in the final score (Welk, \& Wood, 2000). A limitation in using this instrument was that all items are equally scored and no level of physical activity was graded in terms of amount of energy spent doing the activity, nor the amount of time spent on each activity (Welk, \& Wood, 2000). However, it gave an idea of which children were active or inactive, and created a profile for the times of day to help identify when they were least active, so implementation programs can address the activity levels at those times.

Despite these limitations, the PAQ-C has been successfully used with children in grades four and higher and from ages 9-15. Crocker et al. (1997) examined the feasibility of using this measure of physical activity among children. Results indicated that the instrument had acceptable item and test score characteristics in item distribution, and was determined to have good internal consistency. Using a sample of 84 children ages 9-15 years Crocker et al. (1997) found acceptable levels of test-re-test reliability for both males $(r=.75)$ and females $(r=.82)$.

The PAQ-C instrument examined activities for the week, and included such activities at recess, in PE class, lunch (apart from eating), after school. It is simple and easy to read for this age group. All 276 study subjects were asked to complete the PAQ-C and return it to the researcher or CP in their school. This activity took less than 10 minutes. For the regression analyses, activity level was coded as; 


$$
\begin{aligned}
& \circ \quad 0=\text { physical activities performed more than } 2 \text { times per week (no risk). } \\
& \circ \quad 1=\text { activities performed less than } 2 \text { times per week (risk). }
\end{aligned}
$$

Dietary Intakes

Dietary intakes were measured by questioning each student on all the food and beverages they consumed for the past 24 hour, the amounts, methods of preparation and location were recorded by the researcher (Appendix N). Data were used to determine associations between fiber intake, types of carbohydrates (refined and complex) and types of dietary fat on risk factors for T2DM and CVDs among Jamaican adolescents. The 24hour recall is one of the most common methods used in surveys. Its limitations include the reliance on self-reports and memory problems (Forshee, 2004). Also individuals may not be truthful in their reports. Recalling portion sizes is also a problem with the 24 hour recall. In this study each student was interviewed face to face by the researcher who recorded their information in a booklet (Appendix $\mathrm{N}$ ).

The researcher used the United States Department of Agriculture (USDA) multiple-pass method of collecting food intake data. This method employed a five-step approach to ensure accurate and complete recall of food intake, and reduced the respondent's burden. The method is also available in an automated computer version but for this study, the researcher used the five steps verbally to record the data (Appendix N). The five steps are described below:

1. The researcher asked the subjects to tell her all the foods and beverages he/she consumed for the previous day and recorded these in a booklet (Appendix N). 
2. The researcher probed for foods that are normally forgotten such as those eaten between meals.

3. The researcher asked subjects about the time, eating occasion and location where these foods were eaten.

4. The researcher asked the subject to describe in detail the amounts eaten, methods of preparation such as fried or grilled, and to describe the type of food. For example what type of bread (whole wheat as against white bread). The researcher showed subjects pictures of estimates of foods in cups and their equivalents in ounces to determine the amounts of foods eaten

5. The researcher probed for any item that the subject might have left out in step one.

The USDA multi-pass method of data collection has been used in many other studies to include the National Health and Examination Survey, and the WWEIA (What We Eat In America) surveys. In a smaller study, this method effectively assessed food intakes of obese and non-obese women in the US (Conway, Ingwersen, \& Vinyard et al., 2003). In a study of 49 women aged 21-65 years with BMI ranging from $20-40 \mathrm{~kg} / \mathrm{m}^{2}$, the multiple pass method effectively assessed mean energy intake within $10 \%$ of mean actual intake when compared to the previous day. In that study, women overestimated their energy and carbohydrate intakes by $8-10 \%$. However, no significant differences were found between mean actual and recalled intakes of energy and the macronutrients among the obese group. Normal weight and overweight women significantly $(p<.01)$ overestimated their energy, protein and carbohydrate intakes (Conway, et al., 2003). 
In another study comparing observed food intake at a facility with the multiple pass method conducted via telephone the following day, Conway, et al. (2004) found no significant differences between actual and re-called intakes of energy (3294 \pm 111 and $3541 \pm 124 \mathrm{kcal} /$ day). No significant differences were found for the individual macronutrients. Forty-two men ranging from age 21 to 65 with BMI of $21-39 \mathrm{~kg} / \mathrm{m}^{2}$ participated in that study. The energy intake of both obese and non-obese men significantly correlated with estimated energy requirements $(r=.57, p<.05)$. It was concluded that the multiple-pass method of food intake collection can be used successfully to assess intakes of energy, and macronutrients regardless of BMI status.

Comparison of a 24 hour recall against three days of re-call was pilot tested prior to this study to determine if the 24 hour recall represented habitual macronutrient intakes. Strong correlations were found for energy (kcals) $(r=.494, \mathrm{p}<.002)$ and carbohydrate $(\mathrm{g})$ $(r=.427, p<.008)$ intakes between the 24 hour recall and the three days recall in the pilot study. Therefore the 24-hour recall for macronutrient intakes was used in this study. Approximately 10 minutes per student were used in collecting the 24 hour recall in the pilot study which revealed that students ate similar items and with very little variety.

Data on macronutrients intakes in our study were analyzed using the USDA National Nutrient Database for Standard Reference (USDA, Agricultural Research Service, 2005). Cultural food items not found in the USDA National Nutrient Database for Standard Reference were analyzed using the Food Composition Tables, Caribbean Food and Nutrition Institute. Analysis of food intake concentrated mainly on the macronutrients since research has shown a marked transition in the diets of people of the developing world (Popkin, 2001). It was expected that the diets of Jamaicans pattern 
included the high fat, high energy-dense foods of Western countries which had an increase in animal foods, added sugars, increased fat consumption, and a decrease in cereal and fiber intake (Popkin, 2001). In many developing countries more than 30 percent of the total energy now comes from fat (Popkin, 2001). Dietary results were analyzed for individual macronutrients (g), energy (kcals), types of carbohydrates (complex and refined) and fats (saturated and unsaturated), and fiber (g). Macronutrient intakes including energy (kcals), total g intakes for carbohydrates (refined and complex), fiber, fat, types of fat (saturated and monounsaturated fats) were measured using continuous variables and factored into correlation analyses.

\section{Data Analyses}

Statistical analyses were performed using SPSS version 17.0 (SPSS Inc., Chicago, IL, USA). Data analyses included descriptive statistics such as means, standard deviations, frequencies and percentages for demographic characteristics of the population and risk prevalence of the risk factors for T2DM and CVDs. The data analyses included testing for the risk factors of T2DM and CVDs using logistic and multiple linear regressions for determining the associations with the demographic and environmental variables (hypotheses (1a-e). Risk factors were coded as $1=$ risk, and $0=$ no risk, and for predicting relationships with WC and WHR and the other risk factors (hypothesis 2a). Cross-tabulations, Chi square and Odds Ratios were used to compare the risk factors by BMI. Other statistical analyses included Pearson Correlations for diet and individual risk factors of T2DM and CVDs (hypotheses 2c-f), between WC and the other risk factors, and between WHR and other individual risk factors of T2DM and CVDs. Spearman 
Correlation was used to determine the relationship between family history of obesity and subject obesity. The Holm's Sequential Bonferroni Method was applied to control for type 1 error for all analyses. A type 1 error occurs when the null hypothesis is rejected and it is actually true resulting in a false positive classification (Holms, 1979).

For hypothesis number three which compared the two methods of blood testing, analyses included Pearson Correlation, a paired samples t-test, testing for percentage bias to control for random error, and the Bland Altman Plot to determine agreement between the two methods of testing. This statistic compared the two methods (finger prick and venipuncture) which assessed the same parameters (TC, FBG, HbAlc). The limit of agreement was set at $2 \mathrm{SD}$ above and below the mean difference (Altman, Bland, 1983; \& Bland, Altman, 1986). The Bland Altman Plot graphed the difference between the two methods of testing by removing the variation between subjects and leaving the measurement error which indicated the difference was normally distributed. Mean differences close to zero with scores reflecting narrow CI suggested good agreement between the two methods.

Sensitivity and specificity were calculated to determine the validity of the methods of blood testing. Sensitivity refers to the proportion of subjects who are correctly classified as having a disease condition. A test having greater sensitivity is less likely to misclassify an individual with a negative result (Monsen, 1993). Specificity measures the proportion of people without the disease or condition who test negative. The "greater the specificity of a test, the less likely that a positive test will misclassify a person." For this reason, higher specificity is preferred in public health research. These calculations were done using the following contingency table: 


\begin{tabular}{l|c|c|}
\cline { 2 - 3 } \multicolumn{1}{c|}{} & \multicolumn{2}{|c|}{ Test Result } \\
\cline { 2 - 3 } & Positive & Negative \\
\cline { 2 - 3 } Yes & A & b \\
\cline { 2 - 3 } No & C & d \\
\hline
\end{tabular}

$$
\begin{aligned}
& \text { Sensitivity }=\frac{\mathrm{a}}{\mathrm{a}+\mathrm{b}} \\
& \text { Specificity }=\frac{\mathrm{d}}{\mathrm{c}+\mathrm{d}}
\end{aligned}
$$

Percentage bias was calculated to control for random error (chance). A precise measure is one which is reproducible. Since the same instruments were used repeatedly on the subjects, we tested within-instrument-reproducibility. To determine the percentage bias of the two methods of blood testing the following formula was used:

$$
\text { finger prick reading - laboratory value X } 100
$$

laboratory value

Glucose analysis was compared for analytical imprecision of $\leq 3.3 \%, \leq 2.5 \%$ for bias and $\leq 7.9 \%$ for total error (Sacks, et al., 2005). The National Cholesterol Education Program recommendation of a 3\% CV for bias for determining total cholesterol concentration (NCEP, 1990; \& NCEP, 1995) was used for comparing percentage bias of TC. Precision for $\mathrm{HbAlc}$ of $<3 \%$, and $2-3 \%$ for established physiological limits and 
3-4\% for the National Glycohemoglobin Standardization Program (Schwartz et al., 2005) were used.

\section{Research Questions}

1. What is the relationship between ethnic background, gender, place of residence, and income and the risk factors for T2DM and CVDs among Jamaican adolescents?

2. What is the prevalence of risk factors for developing T2DM and CVDs among Jamaican adolescents?

3. Is there a relationship between blood parameters such as $\mathrm{FBG}, \mathrm{TC}$, and $\mathrm{HbAlc}$ measured by finger prick method and the same measures using venipuncture?

\section{Objectives of the Study}

1. To determine the prevalence of the risk factors of T2DM and CVDs (FBG, HbA1c, TC, systolic and diastolic blood pressure, BMI, WC, WHR, positive family history of T2DM, CVDs, and overweight, AN, PA, and dietary intakes of macronutrients) and the relationships between the risk factors for T2DM and CVDs and ethnicity (Blacks, Indians, Mixed); gender (male and female); place of residence (rural, urban); and income among Jamaican adolescents

2. To determine the frequency, cluster, and associations of the risk factors of T2DM and CVDs in Jamaican adolescents using measures such as FBG, HbA1c, TC, systolic and diastolic blood pressure, BMI, WC, WHR, positive family history of T2DM, CVDs, and overweight, AN, PA levels, and dietary intakes of macronutrients. 
3. To compare the results of blood parameters using finger prick techniques with that of venipuncture to determine if there is a relationship between the two methods and determine if the finger prick method can be used instead of venipuncture in the early screening of T2DM and cardiovascular diseases. 
Table 7

Statistical Analyses






\begin{tabular}{|c|c|c|}
\hline Hypotheses & Types of Variables & Statistics \\
\hline $\begin{array}{l}\text { 2. Overweight and obese } \\
\text { Jamaican adolescents will report } \\
\text { high levels of at least one of the } \\
\text { following risk factors: FBG, } \\
\text { HbAlc, TC, BP, waist } \\
\text { circumference, WHR, and } \\
\text { presence of AN, low PA, and } \\
\text { family history of overweight, } \\
\text { CVDs, and T2DM. }\end{array}$ & $\begin{array}{l}\text { Exposure: BMI } \\
\text { Categories (normal, } \\
\text { overweight, obese). } \\
\text { Outcomes: } \\
\text { Categorical variables; } \\
\text { BP and family history } \\
\text { of T2DM and CVDs, } \\
\text { AN, and PA. } \\
\text { Continuous } \\
\text { Variables: } \\
\text { FBG, HbAlc, TC, } \\
\text { Parents' BMI, Waist } \\
\text { Circumference, } \\
\text { WHR, and total } \\
\text { number of risk } \\
\text { factors. }\end{array}$ & $\begin{array}{l}\text { Chi square tests. } \\
\text { Odds ratios } \\
\text { Logistic regression } \\
\text { analyses to determine } \\
\text { associations between } \\
\text { BMI and various } \\
\text { combinations } \\
\text { of risk factors. } \\
\text { Holm's Sequential } \\
\text { Bonferroni Method }\end{array}$ \\
\hline $\begin{array}{l}\text { 2a. Waist circumference and } \\
\text { WHR for Jamaican adolescents } \\
\text { will be positively associated } \\
\text { with the total number of risk } \\
\text { factors for T2DM and CVDs } \\
\text { (high FBG, HbA1c, TC, BP, } \\
\text { subjects' BMI, and presence of } \\
\text { AN, and low PA, and family } \\
\text { history of overweight, CVDs, } \\
\text { and T2DM, and with each } \\
\text { individual risk factor. }\end{array}$ & $\begin{array}{l}\text { Exposure: Waist } \\
\text { Circumference and } \\
\text { WHR } \\
\text { Outcomes: } \\
\text { Categorical variables; } \\
\text { BP and family history } \\
\text { of T2DM and CVDs, } \\
\text { AN, and PA. } \\
\text { Continuous variables: } \\
\text { FBG, HbA1c, TC, } \\
\text { subjects' BMI, } \\
\text { Parents' BMI, some } \\
\text { family history of } \\
\text { CVDs, and T2DM, } \\
\text { and total number of } \\
\text { risk factors. }\end{array}$ & $\begin{array}{l}\text { Pearson Correlations } \\
\text { of WC and WHR with } \\
\text { the total number of } \\
\text { risk factors and with } \\
\text { each individual risk } \\
\text { factor for T2DM } \\
\text { and CVDs. } \\
\text { Holm's Sequential } \\
\text { Bonferroni Method. }\end{array}$ \\
\hline
\end{tabular}




\begin{tabular}{|c|c|c|}
\hline Hypotheses & Types of Variables & Statistics \\
\hline $\begin{array}{l}\text { 2b. Jamaican adolescents with } \\
\text { family history of obesity } \\
\text { (parents' BMI) will present with } \\
\text { > BMI than those without } \\
\text { family history of obesity as } \\
\text { determined by anthropometric } \\
\text { measures and self-reported data. }\end{array}$ & $\begin{array}{l}\text { Exposure: Parents' } \\
\text { BMI based on scale } \\
\text { of } 1-9 \text { silhouettes } \\
\text { corrected to BMI } \\
\text { equivalents. } \\
\text { Outcome: } \\
\text { Actual BMI of } \\
\text { subjects. }\end{array}$ & $\begin{array}{l}\text { Spearman Correlations } \\
\text { between parents' BMI } \\
\text { and subjects' BMI }\end{array}$ \\
\hline $\begin{array}{l}\text { 2c. Energy intakes of Jamaican } \\
\text { adolescents will be positively } \\
\text { associated with the total number } \\
\text { of risk factors for T2DM and } \\
\text { CVDs (high levels of FBG, } \\
\text { HbA1c, TC, BP, waist } \\
\text { circumference, WHR, and } \\
\text { presence of AN, low PA, and } \\
\text { family history of overweight, } \\
\text { CVDs, and T2DM) and with } \\
\text { each individual risk factor. }\end{array}$ & $\begin{array}{l}\text { Exposure: Energy } \\
\text { intakes (total calories } \\
\text { from fat, protein and } \\
\text { carbohydrates) } \\
\text { Outcome: } \\
\text { Categorical variables; } \\
\text { BP and family history } \\
\text { of T2DM and CVDs, } \\
\text { AN, and PA. } \\
\text { Continuous variables: } \\
\text { FBG, HbAlc, TC, } \\
\text { subjects' BMI, } \\
\text { Parents'BMI, some } \\
\text { family history of } \\
\text { CVDs, and T2DM, } \\
\text { and total number of } \\
\text { risk factors. }\end{array}$ & $\begin{array}{l}\text { Pearson Correlations } \\
\text { of energy intake with } \\
\text { the total number of risk } \\
\text { factors and with each } \\
\text { individual risk factor } \\
\text { for T2DM and CVDs. } \\
\text { Holm's Sequential } \\
\text { Bonferroni Method }\end{array}$ \\
\hline
\end{tabular}




\begin{tabular}{|c|c|c|}
\hline Hypotheses & Types of Variables & Statistics \\
\hline $\begin{array}{l}2 \mathrm{~d} \text {. Intakes of complex and refined } \\
\text { carbohydrates of Jamaican } \\
\text { adolescents will be positively } \\
\text { associated with the total number of } \\
\text { risk factors for T2DM and CVDs } \\
\text { (high levels of FBG, HbA1c, TC, } \\
\text { BP, waist circumference, WHR, } \\
\text { and presence of AN, low PA, and } \\
\text { family history of overweight, } \\
\text { CVDs, and T2DM) and with each } \\
\text { individual risk factor. }\end{array}$ & $\begin{array}{l}\text { Exposure: } \\
\text { Carbohydrates } \\
\text { intakes (g) } \\
\text { (complex } \\
\text { and refined). } \\
\text { Outcome: } \\
\text { Categorical } \\
\text { variables;BP and } \\
\text { family history of } \\
\text { T2DM and CVDs, } \\
\text { AN, and PA. } \\
\text { Continuous variables: } \\
\text { FBG, HbA1c, TC, } \\
\text { subjects' BMI, } \\
\text { Parents' BMI, some } \\
\text { family history of } \\
\text { CVDs, and T2DM, } \\
\text { and total number of risk } \\
\text { factors. }\end{array}$ & $\begin{array}{l}\text { Pearson Correlations } \\
\text { of complex and } \\
\text { refined carbohydrate } \\
\text { intakes (g) with the } \\
\text { total number of } \\
\text { risk factors and with } \\
\text { each individual risk } \\
\text { factor for T2DM } \\
\text { and CVDs. } \\
\text { Holm's Sequential } \\
\text { Bonferroni Method }\end{array}$ \\
\hline $\begin{array}{l}\text { 2e. Total fiber intake (g) of } \\
\text { Jamaican adolescents will be } \\
\text { negatively associated with the total } \\
\text { number of risk factors for T2DM } \\
\text { and CVDs (high levels of FBG, } \\
\text { HbA1c, TC, BP, waist } \\
\text { circumference, WHR, and } \\
\text { presence of AN, low PA, and } \\
\text { family history of overweight, } \\
\text { CVDs, and T2DM) and with each } \\
\text { individual risk factor. }\end{array}$ & $\begin{array}{l}\text { Exposure: } \\
\text { fiber intake (g) } \\
\text { Outcome: } \\
\text { Categorical variables; } \\
\text { BP and family history } \\
\text { of T2DM and CVDs, } \\
\text { AN, and PA. } \\
\text { Continuous variables: } \\
\text { FBG, HbA1c, TC, } \\
\text { subjects' BMI, } \\
\text { Parents' BMI, } \\
\text { some family history } \\
\text { of CVDs, and T2DM, } \\
\text { and total number of } \\
\text { risk factors. }\end{array}$ & $\begin{array}{l}\text { Pearson Correlations } \\
\text { of fiber intake }(\mathrm{g}) \\
\text { with the total number } \\
\text { of risk factors and } \\
\text { with each individual } \\
\text { risk factor for T2DM } \\
\text { and CVDs } \\
\text { Holm's Sequential } \\
\text { Bonferroni Method }\end{array}$ \\
\hline
\end{tabular}




\begin{tabular}{|c|c|c|}
\hline Hypotheses & Types of Variables & Statistics \\
\hline $\begin{array}{l}2 \mathrm{f} \text {. Intake of saturated and } \\
\text { unsaturated fat, and intake of } \\
\text { omega- } 3 \text { and omega- } 6 \text { fatty } \\
\text { acids of Jamaican adolescents } \\
\text { will be associated with the total } \\
\text { number of risk factors for } \\
\text { T2DM and CVDs (high levels } \\
\text { of FBG, HbA1c, TC, BP, waist } \\
\text { circumference, WHR, and } \\
\text { presence of AN, low PA, and } \\
\text { family history of overweight, } \\
\text { CVDs, and T2DM) and with } \\
\text { each individual risk factor. }\end{array}$ & $\begin{array}{l}\text { Exposure: } \\
\% \text { caloric intake of } \\
\text { fat (Saturated, } \\
\text { unsaturated). } \\
\text { Outcome: } \\
\text { Categorical variables; } \\
\text { BP and family history } \\
\text { of T2DM and CVDs, } \\
\text { AN, } \\
\text { and PA. } \\
\text { Continuous variables: } \\
\text { FBG, HbA1c, TC, } \\
\text { subjects'BMI, } \\
\text { Parents' BMI, some } \\
\text { family history of } \\
\text { CVDs, and T2DM, } \\
\text { and total } \\
\text { number of risk } \\
\text { factors. }\end{array}$ & $\begin{array}{l}\text { Pearson Correlations of } \\
\text { gram intakes of saturated } \\
\text { and unsaturated fat with } \\
\text { the total number of risk } \\
\text { factors and with each } \\
\text { individual risk factor } \\
\text { for T2DM and CVDs. }\end{array}$ \\
\hline $\begin{array}{l}\text { 3. There will be no significant } \\
\text { differences between FBG, TC, } \\
\text { and HbAlc measured by finger } \\
\text { prick and venipuncture } \\
\text { methods. }\end{array}$ & $\begin{array}{l}\text { Dependent: } \\
\text { FBG, TC and HbAlc, } \\
\text { outcomes for each } \\
\text { variable. } \\
\text { Independent: } \\
\text { Methods of blood } \\
\text { testing. }\end{array}$ & $\begin{array}{l}\text { Two-sided paired sample } \\
\text { t-test }(\alpha=0.05) \text { for mean } \\
\text { differences between the } \\
\text { finger prick and } \\
\text { venipuncture samples. } \\
\text { Pearson correlations. } \\
\text { Bland-Altman plot for } \\
\text { agreement between the } \\
\text { two methods of blood } \\
\text { testing, sensitivity and } \\
\text { specificity for validation of } \\
\text { the tests, and percentage } \\
\text { bias for random error. }\end{array}$ \\
\hline
\end{tabular}




\section{Data Monitoring and Safety}

Protection against Potential Risk

The study was conducted with the permission of Institutional Review Board of Florida International University, the principals of each selected school and the parents of randomly selected students. Participants were informed that they have the right to withdraw from the study at any time. The participants were given a copy of the consent form and the other filed away at Florida International University in locked cabinets.

Study subjects were informed of the purpose of the study, which was to examine Jamaican adolescents for risk of type 2 diabetes and cardiovascular diseases to determine if they are prone to diabetes and cardiovascular disease as seen in younger populations in other countries.

Participants were informed that the study consisted of two parts (screening and a randomly selected group of students) to participate in a smaller study for validation of the methods and they may be asked to participate in both parts. All students were screened for T2DM and CVDs disease risks using finger prick method for determining non-fasting blood glucose levels, TC, and HbAlc. Other measures included blood pressure, presence of AN, anthropometric data such as weight, height, waist circumference, WHR, demographic data, family history of obesity, diabetes, cardiovascular disease, dietary intakes, and physical activity levels.

Confidentiality of personal information and medical data was maintained by the researcher. Only investigators who were directly involved in this study had access to personal information and participants identity were not revealed in any publication from this study. Further confidentiality was maintained by identification of subjects by 
personal identification numbers (PIN) only. Personal identification numbers were assigned from 01-300. The assigned identification numbers linking to subjects were known only to the researcher and kept safely in locked cabinets.

Benefits to Study Subjects.

Since adolescents are prone to obesity and obesity is associated with increased risks for T2DM and CVDs, this study was timely and provided much needed insight into planning intervention programs and encouraged early screening of adolescents to prevent these chronic diseases among this population. Subjects were given a free screening for T2DM and CVDs risks, had their food intakes analyzed, their BMI determined, their level of physical activity assessed and their risk of two debilitating chronic diseases determined. In addition, subjects were given a small compensation for their effort.

\section{Quality Control}

To ensure consistency with the study protocol, students were asked to complete a 12-hour fast before data collection. The researcher questioned the students if they have fasted. Those who have not fasted before blood testing were not tested. To allow for attrition, up to $5 \%$ more subjects than needed to give $80 \%$ power were recruited. High risk subjects were reported to the school nurse as part of the IRB agreement, for followup and discussion with the parents. Each school was given a digital scale and a blood pressure monitor by the researcher to help in the continued monitoring of adolescents' weights and blood pressure. 
Anthropometric data such as weight and height were taken in a secluded room to allow for privacy of the subjects. Weights were taken in light clothing with shoes removed. Scale was disinfected after each use to prevent contamination and recalibrated at intervals. Examinations for AN were done by the school nurse and researcher in a private room (sick bay). All laboratory testing was done by the Central Medical Laboratory in Kingston to reduce variability among laboratory methods. Similarly, all finger prick tests were taken by the health team from the Heart Foundation of Jamaica using the Accu Chek Advantage for FBG and the Accutrend GCT for testing TC. All $\mathrm{HbAlc}$ finger prick tests were performed by the worker from Diabetes Association of Jamaica using the same equipment, the Nycocard manufactured by Axis-Shield.

\section{Summary of Methods}

Based on the evidence that obesity is related to T2DM, CVDs, and that these health conditions are increasing in adolescents, the present study screened for overweight and obesity, and lifestyle factors such as diet and physical activity levels among 276 randomly selected Jamaican adolescents in ten high schools in Jamaica. In addition, biochemical indices such as FBG, TC, and $\mathrm{HbAlc}$ were compared using finger prick and venous methods of 60 randomly selected students from the larger study group. Testing of this sub-sample was done to determine the validity of the finger prick method. Anthropometric measures such as weight, height, body mass index, demographic data, blood pressure, presence or absence of Acanthosis Nigricans (AN), dietary intakes, physical activity levels, family history of overweight and obesity, T2DM, and cardiovascular diseases were also collected. 


\section{CHAPTER IV}

\section{RESULTS}

Jamaican adolescents were studied for the presence of risk factors for T2DM and CVDs. Thirteen risk factors were examined to include subjects' body mass index (BMI), waist circumference (WC), waist-to-hip ratio (WHR), fasting blood glucose (FBG), total cholesterol (TC), glycated hemoglobin (HbAlc), blood pressure (BP), physical activity (PA), food intakes, family history of obesity, type 2 diabetes (T2DM) and cardiovascular diseases (CVDs), and presence of Acanthosis Nigricans (AN). Variables such as gender (male, female), ethnicity, (Blacks, non-Blacks), place of residence (rural, urban), and income levels (low, high) were examined to determine how each related to the risk factors of T2DM and CVDs. Additionally, data were collected on a sub-sample of Jamaican adolescents to compare the methods of blood testing (finger prick versus venipuncture) for screening at field conditions.

\section{Demographic Characteristics}

Of the 300 students who were recruited, 276 participated in the study (112 males and 164 females). Mean age was $15.6 \pm 1.18$ years. Of the 24 non-participants, 4 did not return the parental consent forms, 17 were absent due to an incessant rain experienced during the time of data collection (students were unavoidably absent), and 3 were removed from the data set due to incomplete data. Table 8 shows the demographic makeup of the sample. 
The final sample included more females $(\mathrm{N}=164,59.4 \%)$ than males $(\mathrm{N}=112$, $40.6 \%$ ). Subjects were predominantly Black $(78.2 \%)$ as expected since up to $91 \%$ of the island's population is Black. Only $11(4.0 \%)$ of the participants were Indians. Of the Indian subjects, the majority (9) were females aged 14-16 years and were drawn from the rural schools. The mixed ethnic group comprised $17.0 \%(\mathrm{~N}=47)$ of the sample.

More subjects from the non-traditional $(\mathrm{N}=142,51.4 \%)$ than the traditional $(\mathrm{N}=134,48.6 \%)$ high schools participated in the study. Types of schools were used as a measure of SES. Typically, the traditional schools offer a more academic program and catered to the middle and upper class students. The majority of the sample were in the 16 year age group $(\mathrm{N}=82,29.7 \%)$ followed by $15(\mathrm{~N}=71,25.7 \%)$ and $14(\mathrm{~N}=61,22.1 \%)$. A total of $136(49.3 \%)$ rural and $140(50.7 \%)$ urban subjects participated in the study. 
Table 8

Demographic Characteristics of the Study Population $(N=276)$

\begin{tabular}{|c|c|c|}
\hline Variables & $\bar{n}$ & $\%$ \\
\hline \multicolumn{3}{|l|}{ Gender } \\
\hline Male & 112 & 40.6 \\
\hline Female & 164 & 59.4 \\
\hline \multicolumn{3}{|l|}{ Ethnicity } \\
\hline Black & 216 & 78.2 \\
\hline Indian & 11 & 4.0 \\
\hline Chinese & 1 & 0.4 \\
\hline Caucasian & 1 & 0.4 \\
\hline Mixed & 47 & 17.0 \\
\hline \multicolumn{3}{|l|}{ School-Type } \\
\hline Traditional & 134 & 48.6 \\
\hline Non-traditional & 142 & 51.4 \\
\hline \multicolumn{3}{|l|}{ Age (years) } \\
\hline \multicolumn{3}{|l|}{ (Mean 15.6 \pm 1.2 ) } \\
\hline 14 & 61 & 22.1 \\
\hline 15 & 71 & 25.7 \\
\hline 16 & 82 & 29.7 \\
\hline 17 & 45 & 16.3 \\
\hline 18 & 16 & 5.8 \\
\hline 19 & 1 & 0.4 \\
\hline \multicolumn{3}{|l|}{ Place of Residence } \\
\hline Urban & 140 & 50.7 \\
\hline Rural & 136 & 49.3 \\
\hline \multicolumn{3}{|l|}{ Grade level } \\
\hline 9 & 71 & 25.7 \\
\hline 10 & 78 & 28.3 \\
\hline 11 & 104 & 37.7 \\
\hline 12 & 23 & 8.3 \\
\hline
\end{tabular}


General Description of the Risk Factors

Anthropometric Measures

Table 9 shows frequencies, means, and standard deviations for the anthropometric measures. Heights ranged from $120 \mathrm{~cm}$ to $197 \mathrm{~cm}(168.2 \pm 9.4)$. Weights ranged from 30 $\mathrm{kg}$ to $160 \mathrm{~kg}(67.3 \pm 22.2)$. The overall mean BMI of the study population was $23.8 \pm 7.7$ and ranged from 12.9 to 50.8. More than one-third (42.4\%) of the subjects had normal mean BMI $(20.90 \pm 1.76), 24.6 \%$ of the sample were underweight with mean BMI of $16.72 \pm 1.30$. and $14.9 \%$ were overweight $(\mathrm{BMI}=27.32 \pm 1.38), 50(18.1 \%)$ were obese; class I obese $(n=20, B M I=32.34 \pm 1.69)$, class II obese $(n=19, B M I=36.83 \pm 0.98)$, and class II obese $(\mathrm{n}=11, \mathrm{BMI}=46.21 \pm 8.43)$. When overweight and obese subjects were combined, $33.0 \%(\mathrm{BMI}=35.67 \pm 3.12)$ of the sample was classified as overweight putting them at risk for T2DM and CVDs.

Waist circumferences ranged from $55.0 \mathrm{~cm}$ to $129 \mathrm{~cm}(79.1 \pm 14.3)$. Hip measurements ranged from $68 \mathrm{~cm}$ to $152 \mathrm{~cm}(97.7 \pm 14.7)$. Measures of waist circumference classified $n=197(71.3 \%)$ of the adolescents as not at risk for T2DM and CVDs, $\mathrm{n}=29(10.5 \%)$ had increased risk $(\geq 94 \mathrm{~cm}$ and $\geq 80 \mathrm{~cm}$ for males and females) respectively, and $\mathrm{n}=50(18.1 \%)$ had substantial risk $(\geq 102 \mathrm{~cm}$ and $\geq 88 \mathrm{~cm}$ for males and females). Overall, $28.6 \%$ of the sample had high $\mathrm{WC}$ which classified them at risk for CVDs or T2DM. Most of the sample $(n=255,92.3 \%)$ fell within the normal range for a healthy WHR ( $<1.0$ and $<.85$ for males and females) respectively. Measures of WHR classified only $n=21(7.6 \%)$ subjects at risk for T2DM and CVDs with WHR $\geq 1.0$ and $\geq .85$ for males and females respectively. 
Table 9

Anthropometric Measures of Study Subjects $(N=276)$

\begin{tabular}{|c|c|c|c|}
\hline \multirow[t]{2}{*}{ Risk Factors } & $\mathrm{n}$ & & Mean \pm SD \\
\hline & \multicolumn{3}{|c|}{$\%$} \\
\hline BMI & & & $23.76 \pm 7.72$ \\
\hline Underweight $(<18.5)$ & 68 & 24.6 & \\
\hline Normal (18.5-24.9) & 117 & 42.4 & \\
\hline Overweight $(25.0-29.9)$ & 41 & 14.9 & \\
\hline Obese $(\geq 30)$ & 50 & 18.1 & \\
\hline Height $(\mathrm{cm})$ & & & $168.2 \pm 9.4$ \\
\hline Weight $(\mathrm{kg})$ & & & $67.3 \pm 22.2$ \\
\hline Waist Circumference & & & $79.06 \pm 14.2$ \\
\hline $\operatorname{Normal}(<94 \mathrm{~cm} \mathrm{M},<80 \mathrm{~cm} \mathrm{~F})$ & 197 & 71.4 & \\
\hline Increased risk ( $\geq 94 \mathrm{~cm} \mathrm{M,} \geq 80 \mathrm{~cm} \mathrm{~F})$ & 29 & 10.5 & \\
\hline Substantial risk $(\geq 102 \mathrm{~cm} \mathrm{M}, \geq 88 \mathrm{~cm} \mathrm{~F})$ & 50 & 18.1 & \\
\hline Waist to Hip Ratio & & & $0.80 \pm 0.06$ \\
\hline Healthy $(<.85$ for $\mathrm{F},<1.0$ for $\mathrm{M})$ & 255 & 92.4 & \\
\hline Increased risk ( $\geq .85$ for $F, \geq 1.0$ for $\mathrm{M}$ ) & 21 & 7.6 & \\
\hline
\end{tabular}

$\mathrm{F}=$ female $\mathrm{M}=$ male $\quad \mathrm{BMI}=$ Body Mass Index

Table 10 shows the frequencies, means and standard deviations of selected risk factor indicators (FBG, TC, HbAlc, and BP) for CVDs and T2DM determined by finger prick measures. $39(14.1 \%)$ of the sample was classified at risk with impaired fasting glucose (IFG) that is, FBG $\geq 100$ and $\mathrm{FBG}<126 \mathrm{mg} / \mathrm{dL}$ and $3(1.1 \%)$ classified as diabetic with FBG greater than $126 \mathrm{mg} / \mathrm{dL}$. The total number of subjects classified at risk for T2DM because of high FBG was $n=42(15.2 \%)$. 
Most of the sample ( $\mathrm{n}=250,90.6 \%)$ had normal TC levels with TC less than 170 $\mathrm{mg} / \mathrm{dL}$ according to the National Cholesterol Education Program (NCEP) guidelines for children. However, $8.3 \%$ of the sample was classified at risk for CVDs with TC of $170-$ $200 \mathrm{mg} / \mathrm{dL}$ (borderline). Only three participants had high levels of TC (TC $>200$ $\mathrm{mg} / \mathrm{dL}$ ). For statistical analyses, subjects considered at risk for high TC included those with borderline as well as high levels of TC making a total of $n=26(9.4 \%)$.

The majority of the sample $(n=202,73.2 \%)$ were classified as having normal levels $(<6.5 \%)$ of HbA1c. Results indicated that FBG classified fewer subjects at risk for T2DM than measures of $\mathrm{HbA} 1 \mathrm{c}(\mathrm{n}=42,15.2 \%$ and $\mathrm{n}=74,26.8 \%)$ respectively. Finger prick measures of TC classified $9.4 \%$ of the subjects at risk for T2DM and CVDs as compared to FBG (15.2\%) and HbAlc (26.8\%).

Blood pressure was classified as normal, pre-hypertensive and hypertensive based on the NCEP guidelines which classify children and adolescents taking into account height, gender and age percentiles (Appendices Q and R). Mean systolic and diastolic BP $(\mathrm{mm} / \mathrm{Hg})$ were $116.8 \pm 16.2$ and $69.9 \pm 10.9$ respectively. The majority of the sample ( $\mathrm{n}=205,74.3 \%$ ) was classified as having normal BP which placed them in the $<90^{\text {th }}$ percentile according to their age, gender, and height percentile with measures of systolic and diastolic blood pressure. Twenty-four subjects (8.7\%) were classified as prehypertensive (between the $90^{\text {th }}$ and $95^{\text {th }}$ percentile), and $n=47(17.0 \%)$ were classified as hypertensive (above the $95^{\text {th }}$ percentile). For statistical analyses, subjects who classified in the $90-95^{\text {th }}$ and above the $95^{\text {th }}$ percentile were pooled and classified at risk for high BP $(\mathrm{n}=71,25.7 \%)$. 
Table 10

Biochemical Risk Factors of CVDs and T2DM $(N=276)$

\begin{tabular}{|c|c|c|c|}
\hline Risk Factors & $n$ & $\%$ & Mean \pm SD \\
\hline $\begin{array}{l}\text { Fasting Blood Glucose }(\mathrm{mg} / \mathrm{dL}) \\
\text { Normal FBG }(<100) \\
\text { IFG }(100-126) \\
\text { Diabetes }(>126)\end{array}$ & $\begin{array}{r}234 \\
39 \\
3\end{array}$ & $\begin{array}{r}84.8 \\
14.1 \\
1.1\end{array}$ & $91.21 \pm 10.53$ \\
\hline $\begin{array}{l}\text { Total Cholesterol }(\mathrm{mg} / \mathrm{dL}) \\
\text { Normal }(<170) \\
\text { Borderline }(170-200) \\
\text { Above normal }(>200)\end{array}$ & $\begin{array}{r}250 \\
23 \\
3\end{array}$ & $\begin{array}{r}90.6 \\
8.3 \\
1.1\end{array}$ & $143.0 \pm 21.32$ \\
\hline $\begin{array}{l}\text { HbAlc }(\%) \\
\text { Normal }(\leq 6.5) \\
\text { Above normal }(>6.5)\end{array}$ & $\begin{array}{r}202 \\
74\end{array}$ & $\begin{array}{l}73.2 \\
26.8\end{array}$ & $6.09 \pm 1.33$ \\
\hline $\begin{array}{l}\text { Blood Pressure }(\mathrm{mmHg}) \\
\text { Systolic } \\
\text { Diastolic } \\
\text { Normal }<90^{\text {th }} \text { Percentile } \\
\text { Pre-hypertensive }\left(90^{\text {th }}-95^{\text {th }} \text { Percentile }\right) \\
\text { Hypertensive }\left(>95^{\text {th }} \text { Percentile }\right)\end{array}$ & $\begin{array}{r}205 \\
24 \\
47\end{array}$ & $\begin{array}{r}74.3 \\
8.7 \\
17.0\end{array}$ & $\begin{array}{r}116.8 \pm 16.23 \\
69.9 \pm 10.90\end{array}$ \\
\hline
\end{tabular}

Physical Activity

Physical activity was measured using the Physical Activity Questionnaire for Children (PAQ-C) (Appendix M) which examined activities in and out of school and on weekends. The scale has nine items all rated equally on a five point likert type scale which ranged from no activity to more than 7 times per week. Each question had five responses and was scored from 1-5 where $1=$ inactive (student did not participate in that particular activity at all), 2 = low activity level (activity was performed 1-2 times in the past seven days), $3=$ moderately active (activity was performed 3-4 times in the past seven days, $4=$ active (activity was performed 5-6 times in the past seven days, $5=$ very 
active (activity was performed $>7$ times in the past seven days). An average of all 9 items was taken to determine the activity level of the subject. The mean physical activity score was $2.2 \pm 0.6$ (Table 11) which indicated that subjects participated in physical activities on an average of 1-2 times per week. Low physical activity level was based on an average of two or less times of PA per week. The majority of the subjects $(n=169$, 61.2\%) participated in physical activities more than two times per week compared to 107 $(38.8 \%)$ who participated in physical activities two times or less per week.

Table 11

Physical Activity, Family History of T2DM, CVDS, and Obesity (N=276)

\begin{tabular}{lrcr}
\hline Risk Factors & $\mathrm{n}$ & $\%$ & Mean $\pm \mathrm{SD}$ \\
\hline Physical Activity & & & $2.20 \pm 0.60$ \\
Low $(\mathrm{PA} \leq 2 \mathrm{x} / \mathrm{wk})$ & 107 & 38.8 & \\
High (PA $>2 \mathrm{x} / \mathrm{wk})$ & 169 & 61.2 & \\
Family History T2DM & & & \\
$\quad$ Yes & 145 & 52.5 & \\
No & 131 & 47.5 & \\
Family History CVDS & & & \\
Yes & 82 & 29.7 \\
No & 194 & 70.3 \\
Family History Overweight & & \\
Yes & 181 & 65.6 \\
No & 95 & 34.4 \\
\hline
\end{tabular}

Other measures of physical activity included number of hours per week spent on watching television and playing computer games (Appendix F). Results ranged from 0 to 
84 hours per week $(10.7 \pm 13.5)$ for watching television. Thirty-one subjects $(11.2 \%)$ did not watch television, reportedly they had none. Hours spent on playing computer games ranged from 0 to 60 hours per week $(5.9 \pm 10.3)$. Over one third $(n=106,38.4 \%)$ of the sample did not play computer games.

When asked if they considered themselves to be physically active, the majority of the subjects said yes $(n=211,76.4 \%)$ and $6(2.2 \%)$ of the subjects did not respond. However, when they rated themselves on the PAQ-C (Appendix M), only $61.2 \%$ were physically active.

Table 11 showed that more of the subjects had parents with T2DM $(n=144$, $52.5 \%)$ than CVDs $(\mathrm{n}=82,29.7 \%)$. Family history of overweight and obesity was determined if either parent had BMI $\geq 25.0$ which represented a scale of 5 to 9 on the silhouettes. Results indicated that about two thirds of the sample (65.6\%) reported family history of overweight and obesity.

Presence of Acanthosis Nigricans (AN)

The presence of AN was determined by examining the neck, knuckles, elbows and knees of each subject. The instrument used for determining the presence of AN was developed and used by Burke et al (1999) and comprised different scales for measuring the presence or absence of AN on the neck, elbows, knuckles, and knees (Appendix L). The scale also measured the severity of the condition on the neck and axilla. However, in this study, presence of AN on the axilla was not evaluated. Acanthosis Nigricans is easily recognized by lines of pigmentation on the neck. Therefore, grading of AN was done using pictures which represented the severity of physical signs on the neck 
(Appendix L). For analysis purposes, presence of AN was determined with the neck measures only (Table 12). Acanthosis Nigricans on the neck was graded from 1-4 in severity with grade 1 representing a thin visible line on the neck; grade $2=1-2 \mathrm{~cm}$, grade $3=2-3 \mathrm{~cm}$, and grade four $>3 \mathrm{~cm}$ in width (Acanthosis Nigricans: The Education and Screening Program ANTES).

Table 12

Presence and Severity of Acanthosis Nigricans $(A N)(N=276)$

\begin{tabular}{lrr}
\hline \multicolumn{1}{c}{ Scale } & $\mathrm{n}$ & $\%$ \\
\cline { 2 - 3 } $\begin{array}{c}\text { Presence of AN on neck } \\
\text { Present }\end{array}$ & 122 & 44.2 \\
Absent & 154 & 55.8 \\
Neck Texture & 50 & \\
1 smooth & 30 & 24.0 \\
2 rough & 35 & 28.7 \\
3 coarse & 7 & 5.7 \\
4 extremely coarse & & \\
Knuckles & 47 & 17.0 \\
Present & 229 & 83.0 \\
Absent & & \\
Elbows & 41 & 14.9 \\
Present & 235 & 85.1 \\
Absent &
\end{tabular}

Up to $122(44.2 \%)$ of the adolescents had physical signs of AN as observed on the neck region. Further examination of neck texture revealed that $50(41 \%)$ had visible signs of AN, however, the neck texture was smooth to the touch (grade one). Thirty subjects (24.6\%) had visible signs of AN on the neck with a texture that was just rough to 
the touch (grade two), $35(28.7 \%)$ had grade three AN which represents coarse texture with signs that were highly visible, and 7 (5.7\%) had severe (extremely coarse) AN (grade $4 \geq 3 \mathrm{~cm}$ in width).

Total Number of Risk Factors for T2DM and CVDs

A total of thirteen risk factors were examined. Twelve were coded into risk versus no risk categories and analyzed with logistic regressions. Food intake values were analyzed for total energy and individual macronutrients and their relationship to the risk factors of T2DM and CVDs using Pearson Correlations. For each of the twelve risk factors used in the logistic regressions, 1 represented risk and 0 represented no risk. The following cut-off points were used as at risk for each risk factor: $\mathrm{BMI} \geq 25$, waist circumference (WC $\geq 94 \mathrm{~cm}$ and $\geq 80 \mathrm{~cm}$ for males and females), waist-to-hip ratio (WHR $<1.0$ and $<.85$ for males and females) respectively, fasting blood glucose (FBG $\geq$ $100 \mathrm{mg} / \mathrm{dL})$, total cholesterol $(\mathrm{TC} \geq 170 \mathrm{mg} / \mathrm{dL})$, glycated hemoglobin $(\mathrm{HbAlc}>6.5 \%)$, blood pressure ( $\mathrm{BP}>90^{\text {th }}$ percentile), physical activity ( $\mathrm{PA} \leq 2$ times/week), positive family history of overweight, type 2 diabetes (T2DM) and cardiovascular disease (CVDs), and presence of Acanthosis Nigricans (AN) on the neck.

Table 13 shows that $66.7 \%$ of adolescents had three or more risk factors present $(3.78 \pm 2.32)$. Twelve risk factors were included in the analysis. Results showed the presence of risk factors ranged from 0-10 with one Black, male, rural participant reporting ten out of twelve risk factors for CVDs and/or T2DM. Twelve subjects reported zero risk factors for T2DM and CVDs and 36 reported only one risk factor. No one reported 11 or 12 risk factors. 
Table 13

Number of Risk Factors ${ }^{l}$ for CVDs and/or T2DM $(N=276)$

\begin{tabular}{crrr}
\hline $\begin{array}{c}\text { Number of } \\
\text { Risk Factors }\end{array}$ & $\mathrm{n}$ & \multicolumn{2}{c}{$\begin{array}{c}\text { Cumulative } \\
\%\end{array}$} \\
\hline 0 & 12 & 4.3 & 4.3 \\
1 & 36 & 13.0 & 17.4 \\
2 & 45 & 16.3 & 33.7 \\
3 & 44 & 15.9 & 49.6 \\
4 & 47 & 17.0 & 66.7 \\
5 & 30 & 10.9 & 77.5 \\
6 & 22 & 8.0 & 85.5 \\
7 & 16 & 5.8 & 91.3 \\
8 & 13 & 4.7 & 96.0 \\
9 & 10 & 3.6 & 99.6 \\
10 & 1 & 0.4 & 100.0
\end{tabular}

Risk factors were coded as $1=$ risk and $0=$ no risk based on cut-off points for each risk factor. ${ }^{1}$ Risk factors present ranged from $0-10$. Mean number of risk factors $=3.78 \pm 2.32$

Figure 1 shows a comparison of overweight and obese subjects with normal weight subjects and the distribution of the risk factors. Twelve risk factors were coded and factored into the comparison. The number of risk factors present ranged from 0-10. The sample consisted of more normal weight $(n=185,67 \%)$ than overweight and obese $(n=91,33.0 \%)$ subjects. Sixteen percent $(n=45)$ of the normal weight subjects reported 2 risk factors compared to one overweight subject who reported two risk factors for T2DM and CVDs. As the number of risk factors increased to five or more, more overweight and obese subjects reported greater numbers of risk factors than their nonobese counterparts. Cross-tabulation of the number of risk factors with BMI levels 
revealed that of the 91 subjects with high BMI, $98 \%(n=90)$ had more than 3 risk factors compared to $46 \%$ (93 out of 185 ) of the subjects with normal BMI who reported three or more risk factors for T2DM and CVDs.

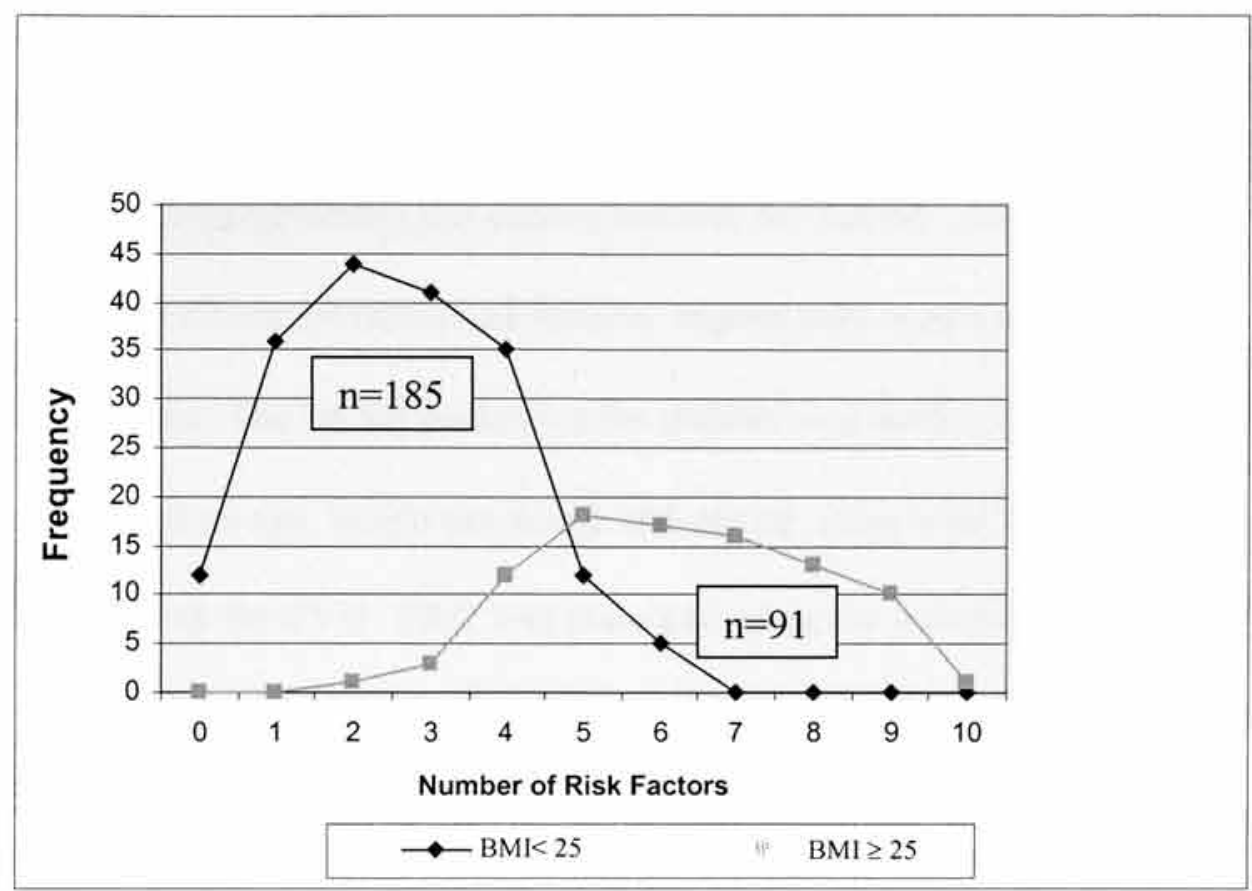

Figure1. Comparison of Overweight and Normal Weight Subjects by Number of Reported Risk Factors for T2DM and CVDs

\section{Number of Risk Factors and Metabolic Syndrome}

The metabolic syndrome (METS) is a cluster of risk factors which include abdominal adiposity, dyslipidemia, impaired glucose tolerance and high blood pressure (Li \& Ford, 2007). Metabolic syndrome is determined by the presence of three or more selected risk factors. According to the National Cholesterol Education Program (NCEP) Adult Treatment Panel III in the USA (2006), presence of any three of the five components of CVDs risk to include central obesity, high blood pressure, high 
triglycerides, low HDL-C, and fasting hyperglycemia constitute the METS. In the current study comparison was made using five selected risk factors; HbA1c, FBG, TC, BP, and central adiposity (high WC) to determine the presence of metabolic syndrome.

High FBG (>100 mg/dL) according to the ADA (2005) guidelines, and HbAlc $>6.5 \%$ based on the International Federation of Diabetes (Nakamota, 2006) were used as surrogates for hyperglycemia and subsequent risk for T2DM. Adult standards of WC $\geq 94 \mathrm{~cm}$ and $\geq 80 \mathrm{~cm}$ for males and females respectively were used for determining central adiposity. The NCEP guidelines for children and adolescents of BP $\geq 90^{\text {th }}$ percentile based on age, height percentile and gender along with $\mathrm{TC} \geq 170 \mathrm{mg} / \mathrm{dL}$ were considered at risk for CVD. BMI was classified using the International Obesity Task Force (IOTF) standard classification where BMI of $\geq 25$ indicated overweight.

A cross comparison of these five risk factors and BMI indicated that $31(34.1 \%)$ of the subjects with high BMI had 3 or more of these five selected risk factors compared to $7(3.78 \%)$ subjects with normal BMI who also had three or more risk factors. Overall, one third of the overweight subjects were classified as having metabolic syndrome with 3 or more of the five selected risk factors. When normal weight and overweight subjects were combined, $13.7 \%$ of the overall sample had three or more of the five selected risk factors.

\section{Results Relating to Hypotheses Testing}

Results of Hypothesis One

Hypothesis one stated that gender, ethnicity, place of residence, and income would be positively associated with the number of risk factors for T2DM and CVDs 
(high levels of FBG, HbA1c, TC, BP, parents' BMI, waist circumference, WHR, and presence of AN, low PA, and family history of CVDs, and T2DM) and with each individual risk factor. Logistic regression analyses (Table 14) showed significance for all four demographic variables and certain risk factors $(\mathrm{p}<.05)$.

Gender predicted FBG $(p=.040)$, WC $(p=.041)$, AN $(p=.013)$, PA $(p<.001)$, family history of CVDs $(\mathrm{p}=.030)$, and total number of risk factors $(\mathrm{p}=.010)$. Males had significantly higher FBG compared to females. However, females were more at risk for high WC, low PA, presence of AN, family history of CVDs, and higher numbers of risk factors. Ethnicity was significant only for predicting family history of T2DM (p=.019) with more Blacks ( $\mathrm{n}=103,71.5 \%$ ) reporting family history of T2DM compared to nonBlacks $(n=41,28.5 \%)$. Place of residence significantly predicted BMI $(\mathrm{p}=.018)$, WC $(\mathrm{p}=.021), \mathrm{PA}(\mathrm{p}=.032)$, and family history of obesity $(\mathrm{p}=.01)$. Urban subjects had significantly higher BMI, higher PA and higher WC, but lower family history of overweight/obesity.

Household income was significant for predicting TC $(p=.039)$, family history of CVDs $(p=.016)$, and family history of obesity $(p=.014)$ (Table 14). Subjects from lower income families had significantly higher levels of TC compared to subjects from higher income families. Subjects from the higher income group reported significantly more family history of CVDs and overweight/obesity. However, when controlled for type 1 error using the Holm's Sequential Bonferroni Method, based on the large numbers of tests performed, results were significant only for gender and PA in the logistic regression. Of the number of subjects who were at risk for low PA, a higher percentage of females 
$(\mathrm{n}=79,48.2 \%)$ were physically inactive than males $(\mathrm{n}=28,25.0 \%)$. Females were significantly more at risk for low physical activity than males, $\mathrm{p}<.001$ (OR 2.7, 95\% CI 1.6 to 4.76$)$.

Gender was also significant for the total number of risk factors $(p=.010)$ in the multiple regression. An equal number (6) of male and female reported zero risk factors. Similarly, 18 males and females reported one risk factor, and slightly higher number of males (25 vs. 20) reported two risk factors for T2DM and CVDs. Females dominated as the number of risk factors increased from 3 to 9 . However, one male reported 10 risk factors. Cross-tabulation of gender and number of risk factors showed more females $(\mathrm{n}=$ $120,73.2 \%)$ had $\geq 3$ risk factors compared to males $(n=63,52.25 \%)$. 
Table 14

Relationships among Gender, Ethnicity, Place of Residence, Income and Risk Factors of T2DM and CVDs

\begin{tabular}{|c|c|c|c|c|c|c|c|}
\hline \multirow[t]{2}{*}{$\begin{array}{l}\text { Risk } \\
\text { Factors }\end{array}$} & \multirow{2}{*}{$\begin{array}{l}\text { Model } \\
\text { P value }\end{array}$} & \multicolumn{3}{|c|}{$\begin{array}{l}\text { Gender } \\
\text { (Male) }\end{array}$} & \multicolumn{3}{|c|}{$\begin{array}{c}\text { Ethnicity } \\
\text { (Non-Black) }\end{array}$} \\
\hline & & OR & $95 \% \mathrm{CI}$ & $\mathrm{P}$ value & OR & $95 \% \quad \mathrm{CI}$ & $P$ value \\
\hline High FBG & .332 & 2.01 & $1.03-3.93$ & .040 & 0.86 & $0.37-2.0$ & .729 \\
\hline High TC & .036 & 0.61 & $0.25-1.5$ & .281 & 0.36 & $.07-1.45$ & .141 \\
\hline High BMI & .100 & 0.64 & $0.37-1.09$ & .101 & 1.03 & $0.55-1.93$ & .917 \\
\hline High WC & .043 & 0.56 & $0.32-0.97$ & .041 & 0.88 & $0.46-1.69$ & .708 \\
\hline Presence of AN & .080 & 0.53 & $0.32-0.87$ & .013 & 0.66 & $0.36-1.21$ & .175 \\
\hline Low PA & $<.001$ & 0.36 & $0.21-0.62$ & $<.001 *$ & 1.20 & $0.66-2.25$ & .536 \\
\hline${ }^{1}$ Family history T2DM & .007 & 0.70 & $0.43-1.1$ & .163 & 2.09 & $1.13-3.9$ & .019 \\
\hline${ }^{l}$ Family history CVDs & .003 & 0.53 & $0.30-0.94$ & .030 & 1.68 & $0.90-3.1$ & .100 \\
\hline${ }^{1}$ Family history Obesity & .014 & 1.05 & $0.64-1.78$ & .844 & 0.62 & $0.34-1.16$ & .133 \\
\hline Total \# of risk Factors & & & $\beta=-.16$ & $.010^{*}$ & & $\beta=.02$ & .765 \\
\hline
\end{tabular}

1 represents positive family history of disease. Regression model used for total number of risk factors.

* considered significant after controlling for type 1 error. 
Table 14 (cont'd)

Relationships among Gender Ethnicity, Place of Residence, Income and Risk Factors of T2DM and CVDs

\begin{tabular}{|c|c|c|c|c|c|c|c|}
\hline \multirow[t]{2}{*}{$\begin{array}{l}\text { Risk } \\
\text { Factors }\end{array}$} & \multirow{2}{*}{$\begin{array}{l}\text { Model } \\
\text { P value }\end{array}$} & \multicolumn{3}{|c|}{$\begin{array}{c}\text { Place of Residence } \\
\text { (Urban) }\end{array}$} & \multicolumn{3}{|c|}{$\begin{array}{l}\text { Household Income } \\
\text { (Low) }\end{array}$} \\
\hline & & OR & $95 \% \mathrm{CI}$ & $\mathrm{P}$ value & OR & $95 \% \quad C I$ & $\mathrm{P}$ value \\
\hline High FBG & .332 & 0.81 & $.42-1.6$ & .555 & 0.92 & $.53-2.21$ & .829 \\
\hline High TC & .036 & 1.20 & $0.52-2.78$ & .674 & 3.70 & $1.06-12.8$ & .039 \\
\hline High BMI & .100 & 1.87 & $1.1-3.14$ & .018 & 1.03 & $0.58-1.76$ & .955 \\
\hline High WC & .043 & 1.90 & $1.1-3.2$ & .021 & 0.79 & $0.45-1.37$ & .398 \\
\hline Presence of AN & .080 & 0.88 & $0.54-1.44$ & .618 & 0.04 & $0.56-1.59$ & .817 \\
\hline Low PA & $<.001$ & 0.57 & $0.35-0.95$ & .032 & 1.10 & $0 . .66-1.98$ & .633 \\
\hline${ }^{1}$ Family history T2DM & .007 & 1.20 & $0.78-2.08$ & .329 & 0.60 & $0.35-1.02$ & .057 \\
\hline${ }^{1}$ Family history CVDs & .003 & 0.77 & $0.45-1.3$ & .342 & 0.51 & $0.29-0.88$ & .016 \\
\hline${ }^{1}$ Family history Obesity & .014 & 0.54 & $0.32-0.90$ & .019 & 0.49 & $0.27-0.87$ & .014 \\
\hline Total \# of risk Factors & & & $\beta=-.02$ & .684 & & $\beta=-.08$ & .191 \\
\hline
\end{tabular}

represents positive family history of disease. 
Hypothesis Two: Predicting Risk Factors of T2DM and CVDs

It was hypothesized that overweight and obese Jamaican adolescents would have at least one of the following risk factors for T2DM and CVDs: high FBG, HbAlc, TC, BP, WC, WHR, and presence of AN, low PA, family history of obesity (Parent's BMI), CVDs, and T2DM. Cross-tabulation of BMI on each of the individual risk factors of T2DM and CVDs (Table 15) and chi square analyses revealed significant results for WC $(\mathrm{OR}=119.20, \mathrm{p}<.001)$, presence of $\mathrm{AN}(\mathrm{OR}=28.45, \mathrm{p}<.001)$, WHR $(\mathrm{OR}=7.70, \mathrm{p}$ $<.001), \mathrm{TC}(\mathrm{OR}=5.50, \mathrm{p}<.001), \mathrm{BP}(\mathrm{OR}=4.60, \mathrm{p}<.001)$, and family history of T2DM $(\mathrm{OR}=2.83, \mathrm{p}<.001)$. In all cases overweight/obese subjects were more likely to be at risk for T2DM and CVDs. Fasting blood glucose $(p=.076)$, HbA1c $(p=.062)$, physical activity $(\mathrm{p}=.850)$, and family history of CVDs $(\mathrm{p}=.554)$ were not significant predictors of BMI. Family history of obesity $(\mathrm{OR}=1.89, \mathrm{p}=.031)$, was not significant when using the Holm's Sequential Bonferroni Method to control for type one error but was significant at the .05 level of significance.

Waist Circumference, AN, WHR, TC, BP, and family history of T2DM were the most prevalent significant risk factors of T2DM and CVDs for overweight and obese subjects. Overweight/obese subjects were 119 times more likely to have higher WC than their underweight/normal weight counterparts. They were 28 times more likely to present with $\mathrm{AN}$ as observed on the neck region. 
Table 15

Cross-tabulations and Odd Ratios of Individual Risk Factors with BMI Classifications (N=276)

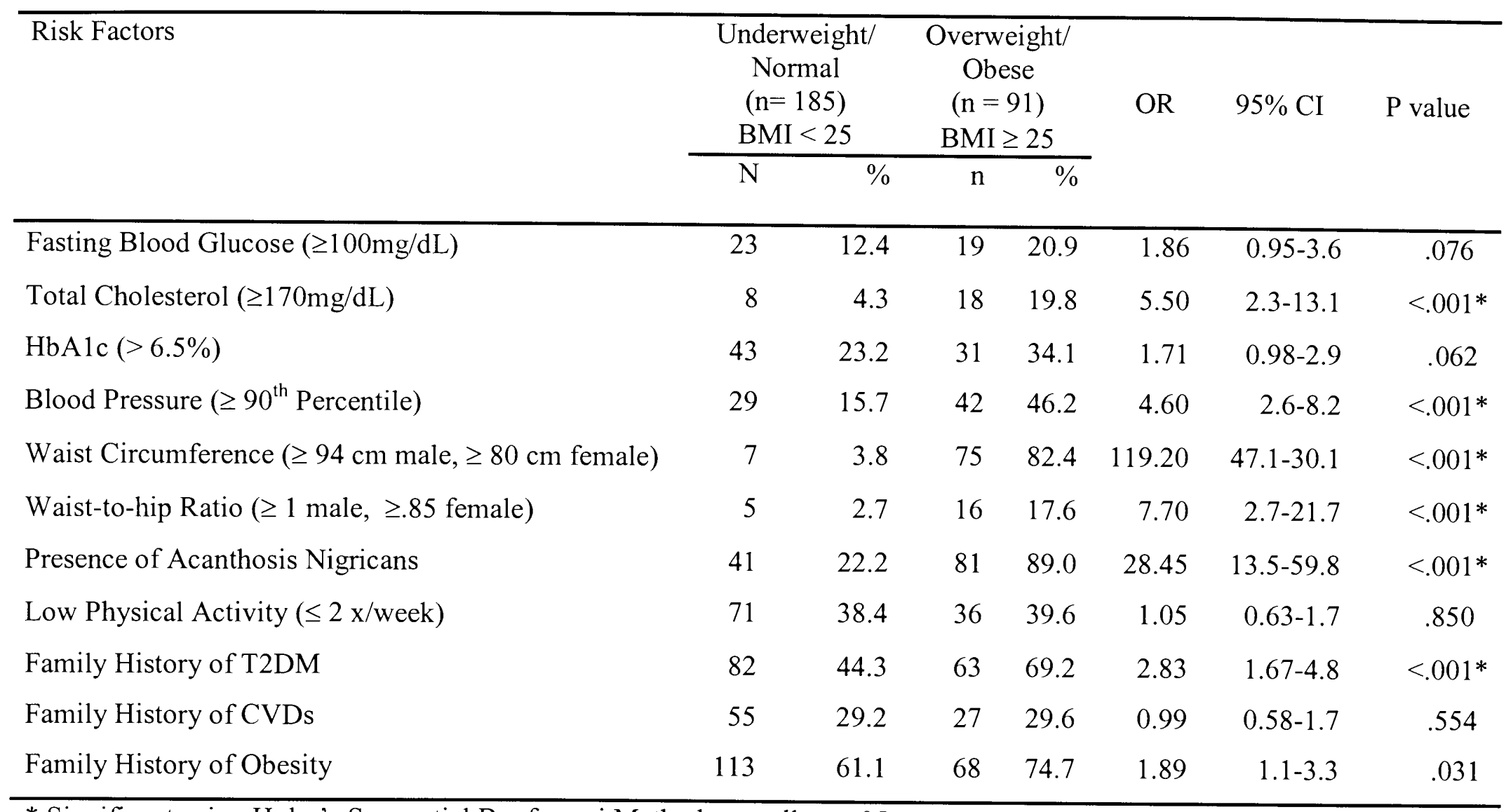

* Significant using Holm's Sequential Bonferroni Method, overall $\alpha=.05$ 
Overweight and obese subjects were 7.7 times more likely to have high WHR, 5.5 times more likely to have high TC, and 4.6 times more likely to have high BP than their underweight and normal weight counterparts. Overweight and obese subjects reported more family history of T2DM, and were 2.8 times more likely to have family history of T2DM (Table 15).

Logistic Regression of the Relationship between BMI and the Risk Factors of T2DM and CVDs

Two logistic regression models were estimated; one with $\mathrm{WC}$ and the other with WHR since the variables were highly correlated with each other. The regression model (Table 16) which included waist-to-hip-ratio, revealed significant results for AN $(\mathrm{OR}=22.80,95 \% \mathrm{CI}=9.8-52.8, \mathrm{p}<.001), \mathrm{WHR}(\mathrm{OR}=9.10,95 \% \mathrm{CI}=2.2-37.6, \mathrm{p}<.001)$, $\mathrm{TC}(\mathrm{OR}=5.30,95 \% \mathrm{CI}=1.5-18.9, \mathrm{p}<.01)$, and family history of $\mathrm{T} 2 \mathrm{DM}(\mathrm{OR}=3.70,95 \%$ $\mathrm{CI}=1.7-8.1, \mathrm{p}<.001)$. With this model, the highest odds of being overweight or obese related to the presence of AN. Subjects with Acanthosis Nigricans were 22.8 times more likely to be overweight/obese. Subjects with high WHR were 9.1 times more likely to be overweight/obese than those with lower WHR. Subjects with high TC $(>170 \mathrm{mg} / \mathrm{dL})$ were 5.3 times more likely to be overweight or obese. Similarly, subjects with family history of T2DM were more likely (3.7 times) to be overweight/obese.

When waist circumference was factored into the regression model, significance was found only for waist circumference $(\mathrm{OR}=53.2,95 \% \mathrm{CI}=17.54-161.1, \mathrm{p}<.001)$, and presence of $\mathrm{AN}(\mathrm{OR}=4.8,95 \% \mathrm{CI}=17-13.9, \mathrm{p}<.01)$. 
Table 16

Logistic Regression Analysis of BMI with Risk Factors for T2DM and CVDs $(N=276)$

\begin{tabular}{|c|c|c|c|c|c|c|}
\hline \multirow[t]{2}{*}{ Risk Factors } & \multicolumn{3}{|c|}{ With Waist Circumference ${ }^{1}$} & \multicolumn{3}{|c|}{ With Waist-to-Hip Ratio ${ }^{2}$} \\
\hline & OR & $95 \% \mathrm{CI}$ & $\mathrm{p}$ value & $\mathrm{OR}$ & $95 \% \mathrm{CI}$ & $\mathrm{p}$ value \\
\hline Fasting Blood Glucose $(\geq 100 \mathrm{mg} / \mathrm{dL})$ & 1.20 & $0.35-4.2$ & .754 & 1.20 & $.46-2.8$ & .764 \\
\hline Total Cholesterol $(\geq 170 \mathrm{mg} / \mathrm{dL})$ & 2.80 & $0.62-12.9$ & .182 & 5.30 & $1.5-18.9$ & $.009 *$ \\
\hline $\mathrm{HbA} 1 \mathrm{c}(>6.5 \%)$ & 1.70 & $0.62-4.76$ & .332 & 1.0 & $0.44-2.4$ & .971 \\
\hline Blood Pressure $\left(\geq 90^{\text {th }}\right.$ Percentile $)$ & 2.20 & $0.78-6.3$ & .137 & 2.90 & $1.3-6.3$ & .010 \\
\hline $\begin{array}{l}\text { Waist Circumference } \\
(\geq 94 \mathrm{~cm} \text { male, } \geq 80 \mathrm{~cm} \text { female })\end{array}$ & 53.20 & $17.54-161.1$ & $<.001 *$ & - & - & - \\
\hline $\begin{array}{l}\text { Waist-to-hip Ratio } \\
(\geq 1 \text { male, } \geq .85 \text { female })\end{array}$ & - & - & - & 9.10 & $2.2-37.6$ & $<.001^{*}$ \\
\hline Presence of AN & 4.80 & $17-13.9$ & $.003 *$ & 22.80 & $9.8-52.8$ & $<.001^{*}$ \\
\hline Physical Activity ( $\leq 2 \mathrm{x} /$ week $)$ & .80 & $0.31-2.9$ & .731 & 0.83 & $0.39-1.7$ & .614 \\
\hline Family History of T2DM & 3.20 & $1.2-8.5$ & .024 & 3.70 & $1.7-8.1$ & $<.001^{*}$ \\
\hline Family History of CVDs & .50 & $.15-1.42$ & .175 & 0.55 & $0.24-1.3$ & .174 \\
\hline Family History of Obesity & 1.40 & $0.49-4.2$ & .510 & 1.30 & $0.57-2.9$ & .542 \\
\hline
\end{tabular}

* Significant using Holm's Sequential Bonferroni Method, overall $\alpha=.05$ for each regression using WC and WHR

${ }^{1}$ Model $\chi^{2}(10, N=276)=219.66, p<.001 \quad{ }^{2}$ Model $\chi^{2}(10, N=276)=157.46, p<.001$ 
Overweight or obese subjects were 53 times more likely to have high WC than their underweight or normal weight counterparts. Overweight or obese subjects were 4.8 times more likely to have AN than their underweight or normal weight counterparts. A comparison of both models revealed that the presence of AN was the strongest predictor of high BMI in the two logistic regressions after WC or WHR (Table 16).

Relationship between WC and WHR and Risk for T2DM and CVDs

Hypothesis 2a stated that waist circumference (WC) and waist-to-hip ratio (WHR) would be associated with the risk factors of T2DM and CVDs. Waist Circumference $\geq$ $94 \mathrm{~cm}$ for males and $\geq 80 \mathrm{~cm}$ for females increased the risk for T2DM and CVDs in adults (Ramchandaran, 2004). Waist-to-hip ratios of 1.0 for males and 0.85 for females or above were used to determine those at risk for T2DM and CVDs. Measures of WC ranged from $55-129 \mathrm{~cm}$ for females and $60-118 \mathrm{~cm}$ for males. The mean WC was 82.5 $\mathrm{cm}$ for males and $76.7 \mathrm{~cm}$ for females. High WC classified $\mathrm{n}=79(28.6 \%)$ of the study subjects at risk for T2DM and CVDs compared to WHR $\mathrm{n}=21(7.6 \%)$ (Table 9). Table 17 shows Pearson Correlation coefficients for WC, WHR and the risk factors of T2DM and CVDs.

Waist circumference positively correlated with BMI $(r=.77, \mathrm{p}<.001)$, total number of risk factors $(\mathrm{r}=.66, \mathrm{p}<.001), \mathrm{BP}(\mathrm{r}=.39, \mathrm{p}<.001)$, and weakly but significantly correlated with FBG $(r=.17, \mathrm{p}=.014)$, family history of obesity $(\mathrm{r}=.16, \mathrm{p}=.008), \mathrm{TC}(\mathrm{r}=.14, \mathrm{p}=.018)$, and family history of T2DM $(\mathrm{r}=-.13, \mathrm{p}=.027)$ However, when controlled for type one error using the Holm's Sequential Bonferroni Method, these weak correlations were no longer considered significant. 
There were positive correlations for WHR and physical activity $(\mathrm{r}=.22, \mathrm{p}<.001)$, $\operatorname{BMI}(\mathrm{r}=.16, \mathrm{p}=.009)$, and total number of risk factors $(\mathrm{r}=.16, \mathrm{p}=.008)$. Larger WC was associated with higher BMI, higher BP, higher FBG, more risk factors, and positive family history of obesity. High WHR was associated with low physical activities, higher BMI, and more risk factors for T2DM and CVDs.

Table 17

Relationship between WC and WHR to Risk Factors of T2DM and CVDs (N=276)

Risk Factors Waist Circumference Waist To Hip Ratio

$\begin{array}{llll}r & p \text { value } & r & p \text { value }\end{array}$

\begin{tabular}{lcccc}
\hline Fasting Blood Glucose (mg/dL) & .17 & $.004^{*}$ & .15 & .011 \\
HbA1c (\%) & .04 & .543 & .02 & .790 \\
Total Cholesterol ( mg/dL) & .14 & $.018^{* *}$ & .06 & .348 \\
Blood Pressure ( percentile) & .39 & $<.001^{*}$ & .07 & .242 \\
BMI & .77 & $<.001^{*}$ & .16 & $.009^{*}$ \\
Acanthosis Nigricans (AN) & .59 & $<.001^{*}$ & .08 & .171 \\
Physical Activity & .06 & .359 & .22 & $<.001^{*}$ \\
Family history of T2DM &. .13 & $.027^{* *}$ & .01 & .897 \\
Family history of CVDs & .02 & .764 & .01 & .931 \\
Family history of Obesity & .16 & $.008^{*}$ & .01 & .937 \\
Total \# of risk factors & .66 & $<.001^{*}$ & .16 & $.008^{*}$
\end{tabular}

* Significant using Holm's Sequential Bonferroni Method, overall $\alpha=.05$ for each set of correlations. Note. AN, PA, family history of T2DM, CVDs, and obesity were coded as $0=$ no risk, $1=$ risk. ${ }^{* *}$ no longer significant after controlling for type one error. 
Relationship between Family History of Obesity and Subjects' Obesity

Hypothesis $2 \mathrm{~b}$ stated that subjects with family history of obesity would be obese.

Family history of obesity was determined by asking students to identify a figure from the silhouettes (Appendix P) using a scale of 1 to 9 which represented 9 levels of BMI categories for both mother and father of the subjects. Scales of 5 and 6 were considered overweight and 7 to 9 represented obesity. Scales were converted to actual BMI levels (Appendix P) and used in the descriptive comparison of parents and subjects' BMI (Figure 2). Subject's BMI was determined by actual measurements of height and weight. Figure 2 shows comparison of subjects' and parents' BMI. Results revealed that there were more overweight and obese mothers $(n=140,52.4 \%)$ than fathers $(n=121$, $47.5 \%)$. There were higher percentages of fathers $(52.5 \%)$ than children $(42.4 \%)$ and mothers $(43.4 \%)$ in the normal weight category. There were some underweight mothers $(\mathrm{n}=11,4.2 \%)$ but no father was reported as underweight. Fathers were not classified as underweight since the cut-off BMI value for underweight was 18.5 and the lowest scale for males represented a BMI of 19.3. Results showed a higher percentage of children $(18.1 \%)$ than mothers $(11.6 \%)$ and fathers $(11.8 \%)$ in the obese category. 


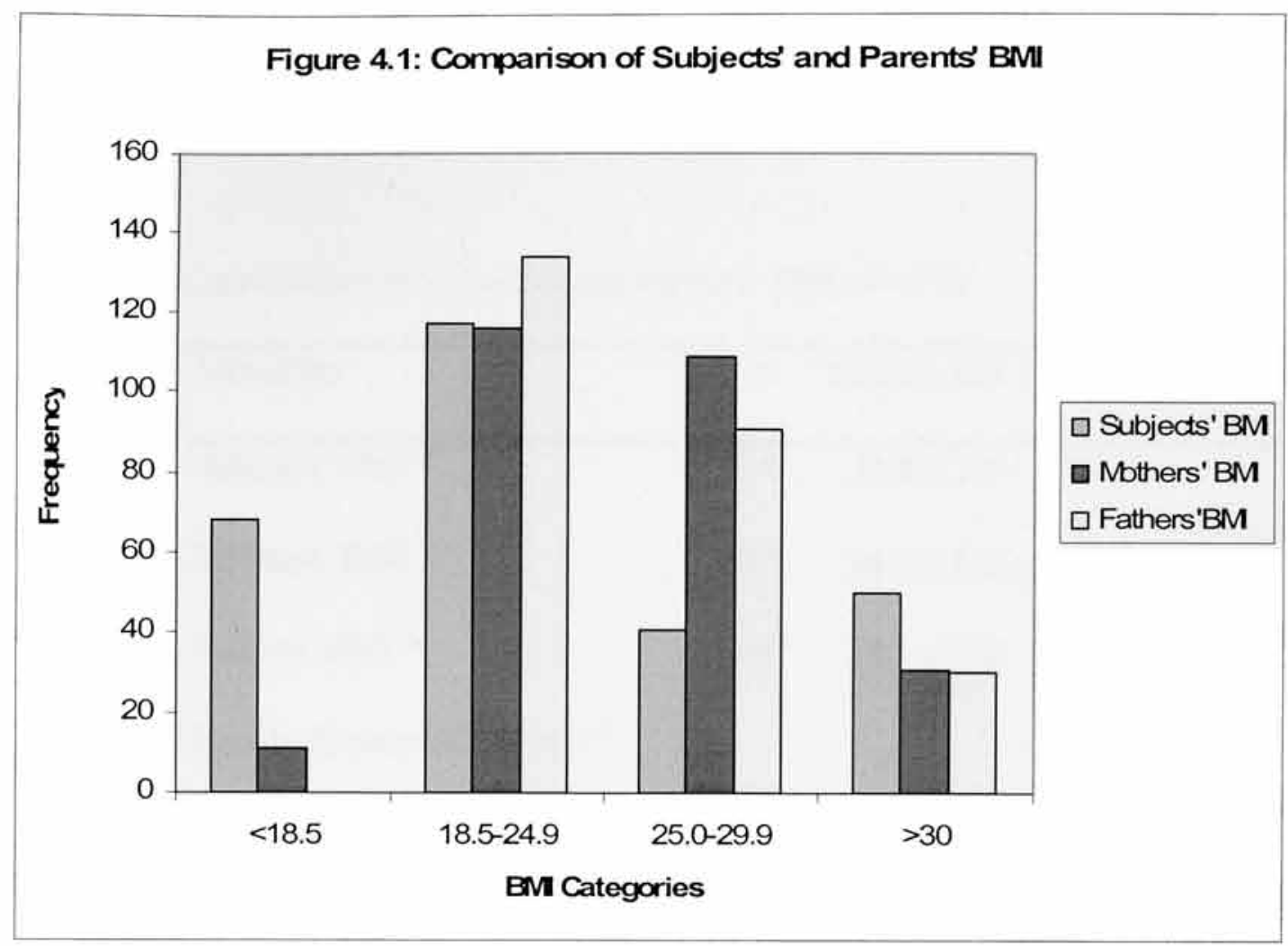

Figure 2. Comparison of Subjects' and Parents' BMI

Family history of obesity was determined if one parent had BMI $\geq 25$. This was coded using categorical variable of 1 for family history and 0 for no family history of obesity. Table 18 and figure 2 show the means, standard deviations and correlations of subjects' and family BMI. Spearman's correlation showed a weak but significant association between family history of obesity and subjects' obesity $(r=.19, \mathrm{p}<.001)$. Positive weak correlations were found between mother and child's BMI $(r=.15, p<.05)$, and between father and child's BMI $(r=.19, \mathrm{p}<.001)$. Mean BMIs were $23.8 \pm 7.7$ for subjects, $23.8 \pm 6.5$ for mothers, and $23.1 \pm 7.4$ for fathers (Table 18). A detailed comparison of BMI categories for subject and parents (Appendix S) was performed. 
Results showed that overweight and obese parents were likely to have children who were also overweight and obese.

Table 18

Spearman Correlations of Subjects' and Parents 'BMI $(N=276)$

\begin{tabular}{lrrrr}
\hline Variables & $\mathrm{n}$ & Mean $\pm \mathrm{SD}$ & $\mathrm{r}$ & $\mathrm{P}$ value \\
& & & & \\
\hline Subjects' BMI $^{\mathrm{a}}$ & 276 & $23.8 \pm 7.7$ & & \\
Mothers' BMI $^{\mathrm{b}}$ & 267 & $24.8 \pm 6.5$ & $.15^{\mathrm{ab}}$ & $.020^{*}$ \\
Fathers' BMI $^{\mathrm{c}}$ & 255 & $23.1 \pm 7.4$ & $.19^{\text {ac }}$ & $.001^{*}$ \\
Family History of Obesity $^{\mathrm{d}}$ & & & $.19^{\text {ad }}$ & $.001^{*}$ \\
\hline
\end{tabular}

Comparison of subjects BMI and family history of obesity coded as $0=$ risk and $1=$ no risk.

\section{Macronutrient Intakes}

Food intakes were measured by a 24-hour recall using the USDA Multiple Pass method. A 24-hour food recall was considered adequate for testing food intakes as demonstrated by the pilot study conducted with a similar population. The pilot study examined the relationship between macronutrient intakes using 24 hour recall compared to 48 and 72 hour recalls. Paired samples t-tests were used to determine if there were significant differences between macronutrient intakes for the 24 hour period compared to 48 and 24 compared to 72 hours. There were no significant differences in gram intakes of carbohydrates $(221 \pm 102)$ for 24 hour recall compared to 48 hours $(224.6 \pm 91.8)$ and between the 24 hour recall and 72 hours of food recall $(243 \pm 85.7)(p>.05)$. Comparison of fat (g) intakes showed no significant differences between 24,48 and 72 hours ( $p>.05$ ). 
Mean fat $(\mathrm{g})$ intakes were $47.6 \pm 23.3,57.4 \pm 51.0$, and $54.4 \pm 37.3$ for the 24,48 and 72 hours respectively. Protein $(\mathrm{g})$ intakes over the same periods were $60.8 \pm 35.8,69.6 \pm$ 48.8 , and $68.6 \pm 35.12$ respectively and no significant differences were found $(\mathrm{p}>.05)$. Fiber $(g)$ intakes showed no significant differences $(p>.05)$ for the 24 hour $(5.35 \pm 3.93)$, to 48 hours $(14.24 \pm 47.45)$ and 24 hour to 72 hours $(11.66 \pm 31.72)$. Total kilocalorie intakes showed no significant differences for 24 hours compared to 48 and 24 to 72 hours of food intakes ( $p>.05)$. Mean kilocalorie intakes were $1564.04 \pm 664.83,1649.22$ \pm 543.67 and $1640.29 \pm 531.48$ for the same time periods. Results of the pilot test indicated that one 24 hour recall of Jamaican adolescents was an acceptable measure of their usual dietary intake of calories, fiber, and macronutrients. No statistically significant differences were noted when comparing 24 hour dietary recall to 48 and 24 hour to 72 hours diet recalls for the select diet parameters.

The relationship between the individual macronutrients, total kilocalories, and fat types were examined. Table 19 shows mean $(\mathrm{g})$ intakes of macronutrients by meals. Overall, food intakes fell within normal ranges for the Acceptable Macronutrient Distribution Ranges (AMDR); for the Dietary Reference Intakes; for the USA and Canada (USDA Dietary Reference Intake, 2002) for all three macronutrients (Table 19). AMDR for carbohydrates is $45-65 \%$ and mean intake of this sample was $58.1 \%$. Recommended fiber intake for 14-18 year old males and females are $38 \mathrm{~g} / \mathrm{d}$ and $26 \mathrm{~g} / \mathrm{d}$ respectively. Overall mean intake of fiber was $14.4 \pm 9.6$. According to the Food and Nutrition Board, Institute of Medicine, National Academies (USDA Dietary Reference Intake, 2002) sugar intake should not be more than $25 \%$ of total energy intake. 
Percentage sugar intake was $15.8 \%$ which fell within the recommended limit. The AMDR for fat is $20-35 \%$ whereas the mean of percentage fat intake in this study was $27.9 \%$. The AMDR for protein is $10-35 \%$ and mean percentage intake was $15.5 \%$ which was within the recommended range for proteins. Intakes of saturated fats were within the normal range. Recommended saturated fat intake is $7-10 \%$ and the mean was reported as $8.3 \%$. However, intakes of unsaturated fats were below the recommended ranges in this sample. Recommended intake of monounsaturated fat is $10-15 \%$ and the reported mean was $9.2 \%$; and polyunsaturated fat intake is $10-35 \%$ and the mean intake was $5.5 \%$ for this sample.

In general, the total kilocalorie intake for 14-18 year olds according to the US and Canadian Dietary Reference Intakes is $2368 \mathrm{kcals} / \mathrm{d}$. However, the mean kilocalorie intake of the adolescent in this study was $1850.4 \pm 869$. Lunch provided the most kilocalories of the day $(647.7 \pm 397 \mathrm{kcals})$. A total of $81(29.3 \%)$ participants missed or skipped breakfast. Fiber intake was low at all three meals with an overall mean daily intake of $14.4 \pm 9.6$. Intakes of refined sugars were highest at the lunch meal $(30.8 \pm 33.3 \mathrm{~g})$ due to the high percentage of carbonated and other beverages that the adolescents had with their lunch meal.

Intakes of omega-3 fatty acids and trans fats were low. A total of $210(76 \%)$ subjects reported eating foods containing omega- 3 fatty acids but at very low intakes compared to 95 who reported consuming foods containing omega- 6 fatty acids. Adequate Intake for omega- 6 is $5-10 \%$ of daily energy intakes and for omega- 3 fatty acids it is $0.6-1.2 \%$. In this sample intakes of both fatty acids were negligible $1.1 \mathrm{~g}$ and $0.4 \mathrm{~g}$ respectively. 
Table 19

Caloric and Macronutrient Intakes Estimated by 24-hour Recall $(N=276)$

\begin{tabular}{lrrrrr}
\hline \multicolumn{1}{c}{ Energy and Nutrient } & \multicolumn{5}{c}{ Mean \pm SD } \\
\cline { 2 - 6 } Intakes & Breakfast & Lunch & Dinner & Snack & 24 hour \\
\cline { 2 - 6 } Total kcals & $319.6 \pm 332.4$ & $647.7 \pm 396.8$ & $593.0 \pm 371.4$ & $289.7 \pm 407.0$ & $1850.05 \pm 869.9$ \\
Total carbohydrate(g) & $59.8 \pm 41.8$ & $94.6 \pm 58.9$ & $88.5 \pm 69.9$ & $42.7 \pm 60.9$ & $268.5 \pm 130.7$ \\
Fiber (g) & $3.0 \pm 3.7$ & $3.6 \pm 3.2$ & $5.8 \pm 6.5$ & $1.8 \pm 2.9$ & $14.2 \pm 9.7$ \\
Sugar (g) & $11.4 \pm 14.6$ & $30.9 \pm 33.3$ & $11.6 \pm 17.0$ & $18.5 \pm 34.2$ & $72.4 \pm 59.3$ \\
Starch (g) & $26.6 \pm 33.8$ & $46.6 \pm 39.3$ & $66.8 \pm 51.1$ & $18.5 \pm 27.0$ & $158.5 \pm 90.7$ \\
Protein (g) & $11.4 \pm 13.6$ & $25.7 \pm 22.0$ & $28.3 \pm 19.2$ & $6.2 \pm 12.2$ & $71.6 \pm 37.8$ \\
Total fats (g) & $12.6 \pm 15.9$ & $18.3 \pm 14.2$ & $14.9 \pm 16.1$ & $11.4 \pm 16.4$ & $57.3 \pm 35.7$ \\
Saturated Fats (g) & $3.6 \pm 4.3$ & $5.7 \pm 4.6$ & $3.4 \pm 3.2$ & $4.5 \pm 7.9$ & $17.1 \pm 11.9$ \\
Monounsaturated Fats (g) & $4.7 \pm 6.7$ & $6.1 \pm 6.2$ & $5.3 \pm 6.5$ & $2.8 \pm 5.3$ & $18.9 \pm 13.5$ \\
Polyunsaturated Fats (g) & $2.9 \pm 4.9$ & $3.2 \pm 3.4$ & $3.8 \pm 5.5$ & $1.4 \pm 2.9$ & $11.3 \pm 9.5$ \\
Omega-3 FA (g) & $0.07 \pm 0.7$ & $0.1 \pm 0.8$ & $0.2 \pm 1.1$ & 0.9 & $0.3 \pm 1.5$ \\
Omeag-6 FA (g) & $0.09 \pm 0.7$ & $0.1 \pm 0.8$ & $0.2 \pm 1.0$ & $0.03 \pm 0.4$ & $0.4 \pm 1.5$ \\
Transfats (g) & $0.03 \pm 0.1$ & $0.1 \pm 0 . .8$ & $0.2 \pm 1.1$ & $003 \pm .0$ & $0.2 \pm 1.1$ \\
Cholesterol (mg) & $64.5 \pm 108.4$ & $59.1 \pm 74.2$ & $75.7 \pm 106.6$ & $10.4 \pm 25.8$ & $139.6 \pm 133.9$ \\
\hline
\end{tabular}


Only 60 subjects reported consuming foods which contained trans fatty acids at very low levels. Mean cholesterol intake (139.6 \pm 133.9$)$ was lower than the suggested $300 \mathrm{mg} / \mathrm{d}$ with $263(95.3 \%)$ of the subjects reported consuming foods with cholesterol (Table 19).

Correlations of Macronutrients and Risk Factors of T2DM and CVDs

Correlations for the macronutrients and the risk factors of T2DM and CVDs were performed. Results indicated significant correlations for some of the risk factors of T2DM and CVDs at the .05 level of significance (Appendices T, U, and V). Although the correlations were significant, none reached moderate effect size ( $r=.3)$ (Cohen, 1988). Consideration was given to the high numbers of risk factors and macronutrients that were compared in our analyses. Therefore, the Holm's Sequential Bonferroni Method (Holm's, 1979) was applied to reduce type 1 error.

Hypothesis $2 \mathrm{c}$ to $2 \mathrm{f}$ examined the correlations between macronutrient $(\mathrm{g})$ intakes and the twelve risk factors studied. It was expected that higher intakes of refined carbohydrates, total fat, types of fats, and total kilocalories would positively correlate with high BMI, WC, and presence of AN. Variables were weakly correlated ranging from -.18 between WC and total fat to .10 between PA and total carbohydrate. After controlling for type 1 error, significance was found only between $\mathrm{WC}$ and total fat intakes $(r=-.18, p=.003)$ and between the presence of AN and fiber intakes $(\mathrm{r}=-.18, \mathrm{p}=.003)$. Lower intakes of fats were associated with higher WC, and lower fiber intake was associated with presence of AN. The risk factors of T2DM and CVDs and types of fats consumed (Appendix V) were not significant after controlling for type 1 error. 
Validation of Finger Prick Method of Blood Testing as a Screening Tool

Results of Hypothesis 3

Hypothesis three stated that there would be no significant differences between FBG, TC, and HbAlc measured by finger prick and venous blood samples. Sixty of the original 276 subjects were randomly selected prior to data collection for participating in the methods of blood testing. One subject was removed from this sub-study sample due to incomplete data set needed for the study. The power analysis using estimates of variances and effect sizes derived from similar studies (Tamborlane, 2005; Schwartz, et al., 2005; McGillis-Bindler 7 Bruya, 2005) indicated that a sample size of 45 was sufficient to detect differences of a medium effect size $(d=.5)$ (Cohen, 1988) between finger prick blood parameters (blood glucose, $\mathrm{HbAlc}$ and TC) and venipuncture technique to yield $90 \%$ power with significance level of .05 for a two-sided paired samples t-test. Table 20 shows the demographic characteristics of the sub-study sample of size 59 .

The sub-study sample consisted of almost equal numbers of subjects by school type, and location. More females $(n=35,59.3 \%)$ than males $(n=24,40.7 \%)$ were randomly selected from all four grade levels $(9-12)$ with the majority $(40.7 \%)$ falling in grade eleven. The mean age was $15.6 \pm 1.19$ years which is identical to the main study sample mean $(15.6 \pm 1.18)$. Majority of the subjects $(n=21,35.6 \%)$ were in the 16 year age group. The sample consisted of mostly Blacks (81.4\%). Sub-study sample was representative of the main study sample. 
Table 20

Demographic Characteristics of Sub-Study Sample $(n=59)$

\begin{tabular}{|c|c|c|}
\hline Variables & $\mathrm{n}$ & $\%$ \\
\hline \multicolumn{3}{|l|}{ Gender } \\
\hline Male & 24 & 40.7 \\
\hline Female & 35 & 59.3 \\
\hline \multicolumn{3}{|l|}{ Location } \\
\hline Urban & 30 & 50.8 \\
\hline Rural & 29 & 49.2 \\
\hline \multicolumn{3}{|l|}{ School-type } \\
\hline Traditional & 29 & 49.2 \\
\hline Non-Traditional & 30 & 50.8 \\
\hline \multicolumn{3}{|l|}{ Grade level } \\
\hline 9 & 15 & 25.4 \\
\hline 10 & 16 & 27.1 \\
\hline 11 & 24 & 40.7 \\
\hline 12 & 4 & 6.8 \\
\hline \multicolumn{3}{|l|}{ Age (years) $(15.6 \pm 1.2)$} \\
\hline 14 & 15 & 25.4 \\
\hline 15 & 10 & 16.9 \\
\hline 16 & 21 & 35.6 \\
\hline 17 & 10 & 16.9 \\
\hline 18 & 3 & 5.2 \\
\hline \multicolumn{3}{|l|}{ Ethnicity } \\
\hline Blacks & 48 & 81.4 \\
\hline Non-Blacks & 11 & 18.6 \\
\hline
\end{tabular}

Table 21 describes the results of selected risk factors for the sub-study sample and the main study group. The sub-study sample was randomly chosen, descriptive data show a higher percentage of subjects from the sub-study sample (13.6\%) were at risk for high TC than those from the main study $(9.4 \%)$. Similar results were found for FBG. Approximately $15 \%$ of subjects from the main study were at risk for high FBG compared 
to $25.4 \%$ from the sub-study sample. Results for $\mathrm{HbAlc}$ showed that a higher percentage of subjects from the main study (26.8\%) had high HbAlc compared to the sub-study sample $(22.0 \%)$. There were no significant differences between the risk factors for the main sample and the sub-study sample which indicated the sub-study sample was representative.

Table 21

Comparison of Sample to Sub-Study Sample: Blood Measures

\begin{tabular}{|c|c|c|c|c|c|c|}
\hline \multirow[t]{3}{*}{ Risk Factors } & \multirow{2}{*}{\multicolumn{3}{|c|}{$\begin{array}{c}\text { Main Study }(\mathrm{N}=276) \\
\text { Finger Prick }\end{array}$}} & \multirow{2}{*}{\multicolumn{3}{|c|}{$\begin{array}{c}\text { Sub-study }(\mathrm{n}=59) \\
\text { Venipuncture }\end{array}$}} \\
\hline & & & & & & \\
\hline & $\mathrm{n}$ & $\%$ & Mean \pm SD & $\mathrm{n}$ & $\%$ & Mean \pm SD \\
\hline $\mathrm{TC}(\mathrm{mg} / \mathrm{dL})$ & & & $143.0 \pm 21.32$ & & & $145.85 \pm 21.61$ \\
\hline Normal $(<170)$ & 250 & 90.6 & & 51 & 86.4 & \\
\hline Borderline $(\geq 170<200)$ & 23 & 8.3 & & 7 & 11.9 & \\
\hline Above Normal $(>200)$ & 3 & 1.1 & & 1 & 1.7 & \\
\hline FBG (mg/dL) & & & $91.21 \pm 10.53$ & & & $95.24 \pm 10.27$ \\
\hline Normal $(<100)$ & 234 & 84.8 & & 46 & 78.0 & \\
\hline IFG $(\geq 100<126)$ & 39 & 14.1 & & 12 & 20.3 & \\
\hline Diabetes $(>126)$ & 3 & 1.1 & & 1 & 5.1 & \\
\hline HbAlc $(\%)$ & & & $6.09 \pm 1.33$ & & & $5.40 \pm .81$ \\
\hline Normal $(\leq 6.5)$ & 202 & 73.2 & & 46 & 78.0 & \\
\hline Above Normal $(>6.5)$ & 74 & 26.8 & & 13 & 22.0 & \\
\hline
\end{tabular}

$\mathrm{FBG}=$ Fasting Blood Glucose HbAlc $=$ Glycated Hemoglobin

Table 22 shows the frequency and Pearson correlations for finger prick versus venipuncture measures for FBG, TC and $\mathrm{HbA1c}$. Finger prick and venous TC were highly correlated $(\mathrm{r}=.80, \mathrm{p}=.001)$. Correlations for FBG approached moderate size effect $(\mathrm{r}=$ $.28, \mathrm{p}=.032)$, whereas, HbAlc measure by both methods of blood testing were weakly and not significantly correlated $(\mathrm{r}=.23, \mathrm{p}=.079)$. 
Table 22

Frequency and Correlations of FBG, TC and HbAlc $(n=59)$

\begin{tabular}{|c|c|c|c|c|c|c|}
\hline \multirow[t]{2}{*}{ Variables } & \multicolumn{2}{|c|}{ Finger prick } & \multicolumn{2}{|c|}{ Venous } & \multirow[b]{2}{*}{$\mathrm{r}$} & \multirow[b]{2}{*}{$\mathrm{p}$ value } \\
\hline & $\mathrm{n}$ & $\%$ & $\mathrm{n}$ & $\%$ & & \\
\hline $\mathrm{FBG}(\mathrm{mg} / \mathrm{dL})$ & & & & & .28 & $.032^{*}$ \\
\hline Normal $(>100)$ & 46 & 78.0 & 41 & 69.5 & & \\
\hline IFG(100-126) & 12 & 20.3 & 17 & 28.8 & & \\
\hline Diabetes $(>126)$ & 1 & 1.7 & 1 & 1.7 & & \\
\hline $\mathrm{TC}(\mathrm{mg} / \mathrm{dL})$ & & & & & .80 & $.001 *$ \\
\hline Normal $(<170)$ & 51 & 86.4 & 41 & 69.5 & & \\
\hline Borderline (170-200) & 7 & 11.9 & 12 & 20.3 & & \\
\hline Above normal $(>200)$ & 1 & 1.7 & 6 & 10.2 & & \\
\hline $\mathrm{HbAlc}(\%)$ & & & & & .23 & .079 \\
\hline Normal $(\leq 6.5)$ & 46 & 78.0 & 54 & 91.5 & & \\
\hline Above normal $(>6.5)$ & 13 & 22.0 & 5 & 8.5 & & \\
\hline
\end{tabular}

$\mathrm{FBG}=$ fasting blood glucose $\quad \mathrm{TC}=$ total cholesterol $\mathrm{HbAl} \mathrm{c}=$ glycated hemoglobin

Table 23 revealed significant differences for finger prick and venous testing for TC $(\mathrm{p}<.001)$ and HbAlc $(\mathrm{p}<.001)$, but not for FBG $(\mathrm{p}=.185)$ using paired samples t-test 
Table 23

Paired Samples t-test for FBG, TC, and HbAlc ( $n=59)$

\begin{tabular}{|c|c|c|c|c|c|}
\hline \multirow[t]{2}{*}{ Variables } & Finger prick & Venous & \multicolumn{3}{|l|}{ Mean Difference } \\
\hline & & Mean \pm SD & & $95 \% \mathrm{CI}$ & $\mathrm{p}$ value \\
\hline $\begin{array}{l}\text { FBG } \\
(\mathrm{mg} / \mathrm{dL})\end{array}$ & $92.88 \pm 11.97$ & $95.23 \pm 10.27$ & $-2.35 \pm 13.45$ & $-5.85,1.15$ & .185 \\
\hline $\mathrm{TC}(\mathrm{mg} / \mathrm{dL})$ & $145.85 \pm 21.6$ & $157.93 \pm 30.0$ & $-12.07 \pm 18.3$ & $\begin{array}{r}-16.85,- \\
7.30\end{array}$ & .001 \\
\hline HbAlc $(\%)$ & $6.08 \pm 1.49$ & $5.40 \pm 0.81$ & $0.67 \pm 1.5$ & $0.27,1.10$ & .001 \\
\hline
\end{tabular}

Comparison of Finger Prick and Venous Blood Testing for Fasting Blood Glucose

Fasting blood glucose (FBG) values ranged from $64.8 \mathrm{mg} / \mathrm{dL}$ to $149.4 \mathrm{mg} / \mathrm{dL}$ for finger prick and $77.4 \mathrm{mg} / \mathrm{dL}$ to $126.0 \mathrm{mg} / \mathrm{dL}$ for venous blood samples. There were two outliers for finger prick FBG compared to one for venous FBG (Appendix W). Finger prick testing of FBG overestimated subjects in the normal blood glucose range whereas venous method classified more subjects with Impaired Fasting Glucose (IFG) than finger prick method (Figure 3). Both methods of testing found only one subject with FBG >126 $\mathrm{mg} / \mathrm{dL}$. Finger prick testing of FBG was less sensitive than venous testing $(33.3 \%)$ in classifying subjects with above normal FBG, and had high specificity (82.9\%). Both methods of testing correctly classified subject with diabetes. 


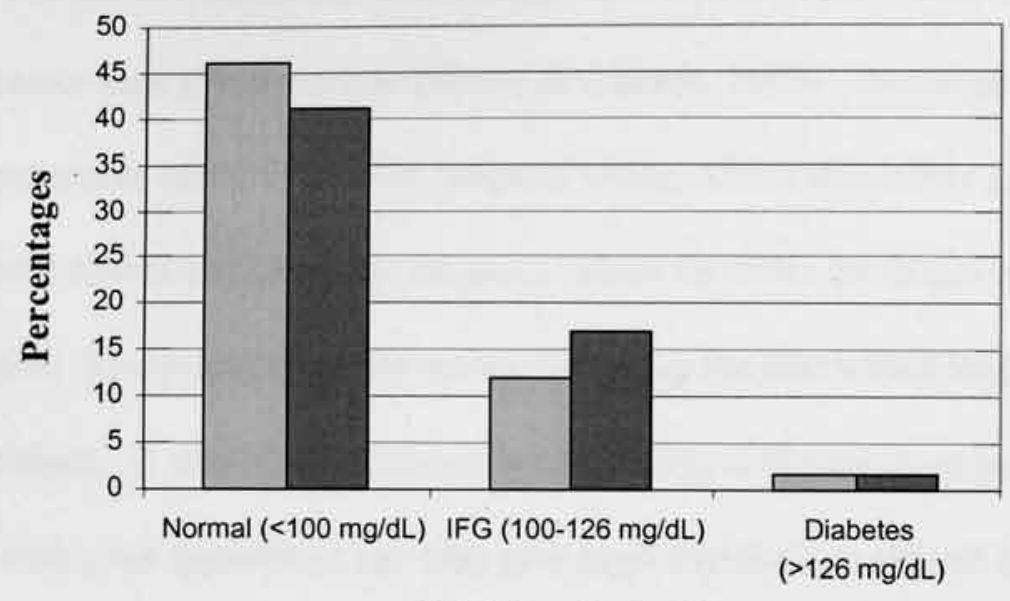

口Finger prick

a Venous

FBG Categories $(n=59)$

Figure 3: Comparison of Finger Prick and Venous Fasting Blood Glucose

Pearson correlation of finger prick and venous FBG was significant $(\mathrm{r}=.28, \mathrm{p}=.032)$, and further testing of FBG using paired samples t-test (Table 23) showed that the means were not significantly different $(\mathrm{p}=.185)$ which indicated that the mean level for finger prick testing of FBG was close to venous FBG. For one method to substitute for another there should be agreement between the two methods, therefore further comparison of finger prick and venous FBG was done using the Bland Altman Plot.

Bland Altman Plot of Agreement for Fasting Blood Glucose

The Bland Altman Plot (Altman, Bland, 1983; \& Bland, Altman, 1986) measured the degree of agreement between two clinical methods. The plot consisted of the difference between the methods plotted against their mean. Correlation coefficients commonly used to compare clinical methods do not necessarily show good agreement 
between the two methods. If the range of values is wide the correlation coefficient will be greater than if it is narrow (Bland \& Altman, 1999). Investigators tend to compare the two measures using the whole range of values which inevitably gives a high correlation.

Figure 4 shows the complete range of values for FBG for finger prick and venous samples. The majority of the scores fell along the line which invariably gives a high correlation. A test of significance is completely different from test of agreement since data with poor agreement can also give high correlations (Bland \& Altman, 1999).

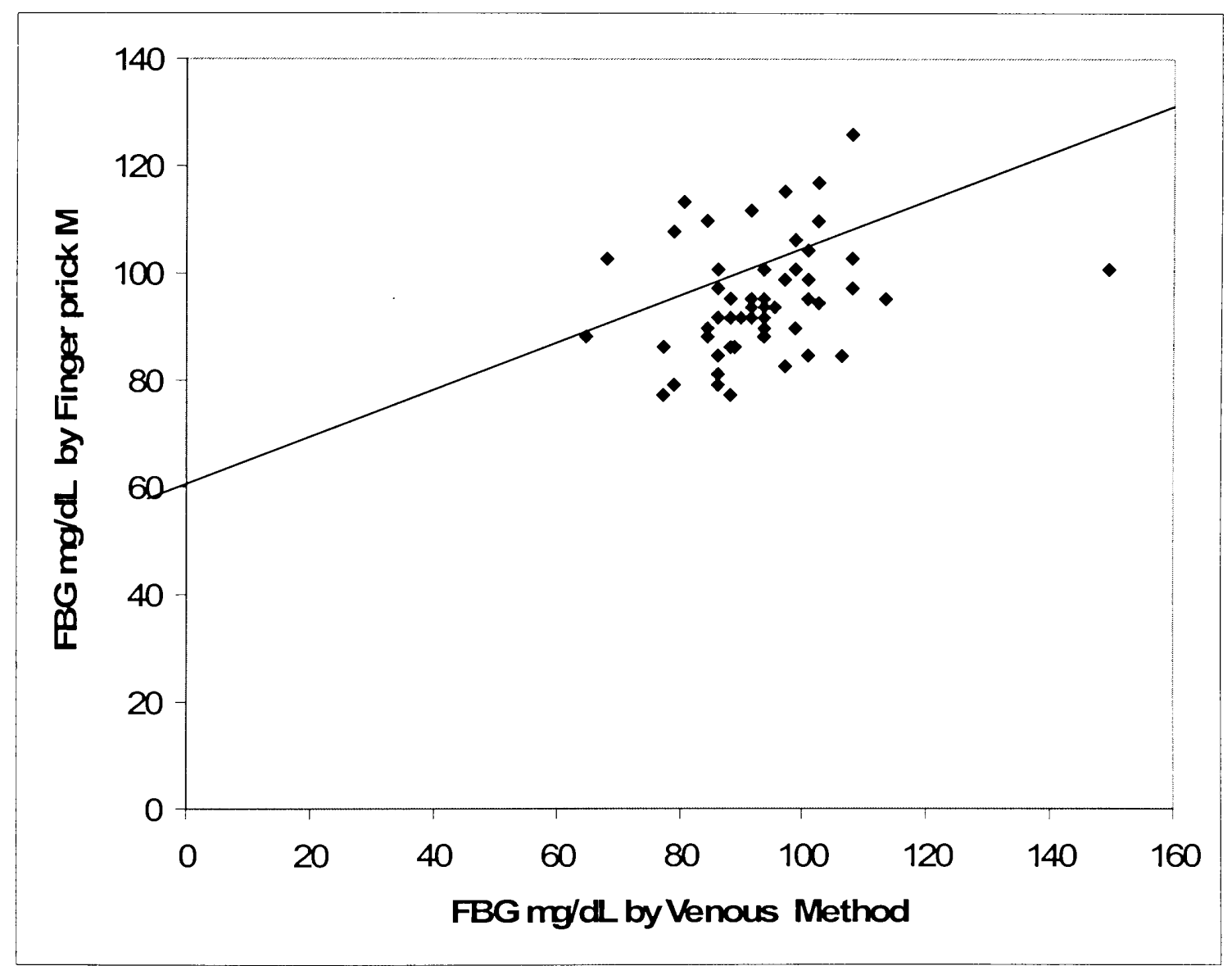

Figure 4. Comparison of Fasting Blood Glucose by Finger Prick and Venous Method $(\mathrm{n}=59)$ 
Figure 5 plots the difference between each capillary whole blood sample for FBG and each corresponding venous sample against the mean of the two samples. Good agreement indicates that the majority of the scores will fall within \pm 2 SD from the mean (Bland \& Altman, 1999). The majority of scores for FBG fell within the \pm 2 SD from the mean. Only two scores were above $+2 \mathrm{SD}$ and only one below $-2 \mathrm{SD}$. Based on paired samples t-test, and the Bland Altman Plot for agreement between methods of blood testing, finger prick testing of FBG appeared to be a good measure for early screening of risk factors for T2DM and CVDS in this sample.

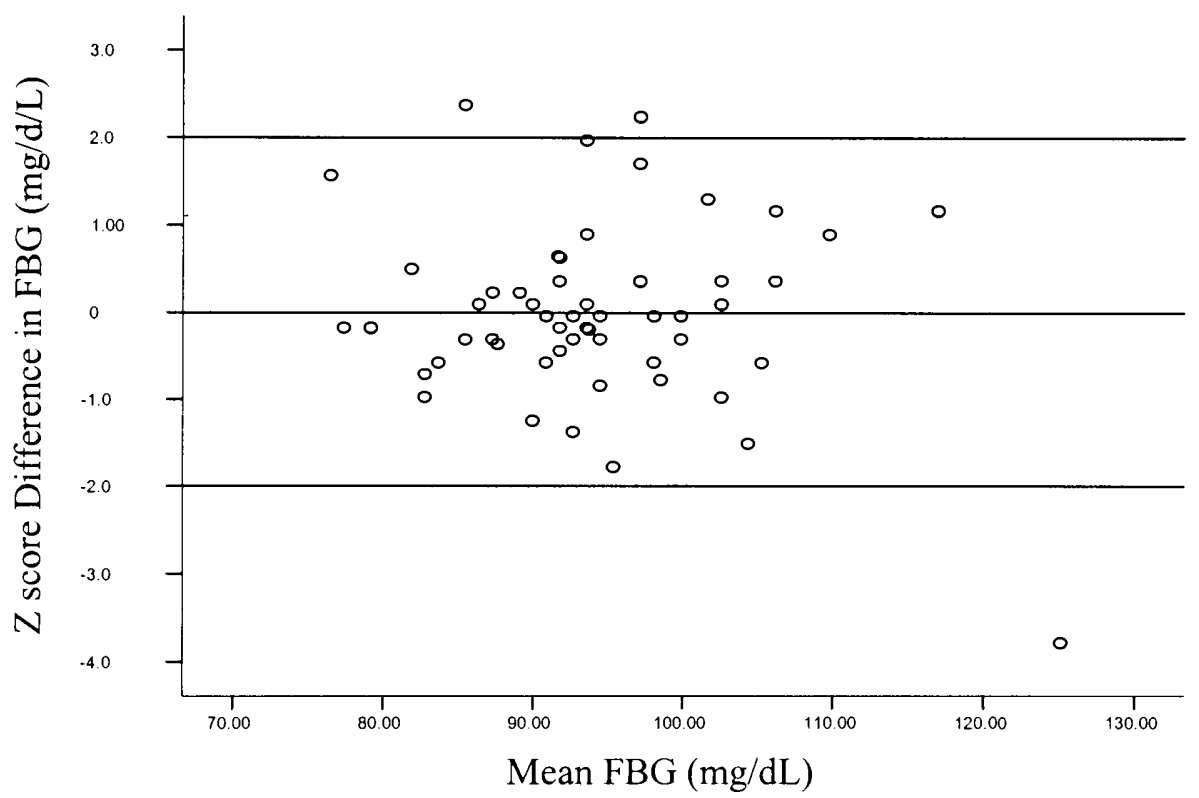

Figure 5. Bland Altman Plot for Finger Prick and Venous Method FBG $(n=59)$

Comparison of Finger Prick and Venous Blood Testing for Total Cholesterol

Total cholesterol values ranged from 123.52 to $203.6 \mathrm{mg} / \mathrm{dL}$ for finger prick and 96.5 to $235.46 \mathrm{mg} / \mathrm{dL}$ for venous blood samples. Strong positive correlation was found between the two measures of TC $(r=.80, p<.001)$. However, further comparison using 
paired samples t-test showed that TC measured using finger prick and venous blood methods were significantly different with a mean difference of $-12.07 \pm 18.3, p<.001$ $(95 \% \mathrm{CI}=-16.85$ to -7.6$)$. Figure 6 showed that venous blood testing identified more subjects with borderline high TC than finger prick. Venous blood testing also identified more subjects with above normal TC than did finger prick.

Finger prick TC had specificity of $100 \%$ and sensitivity of $16.7 \%$. Results indicated that subjects without high TC were correctly classified by the finger prick method. Finger prick TC shows high specificity and high correlations between both methods of testing $(\mathrm{r}=.80, \mathrm{p}<.001)$.

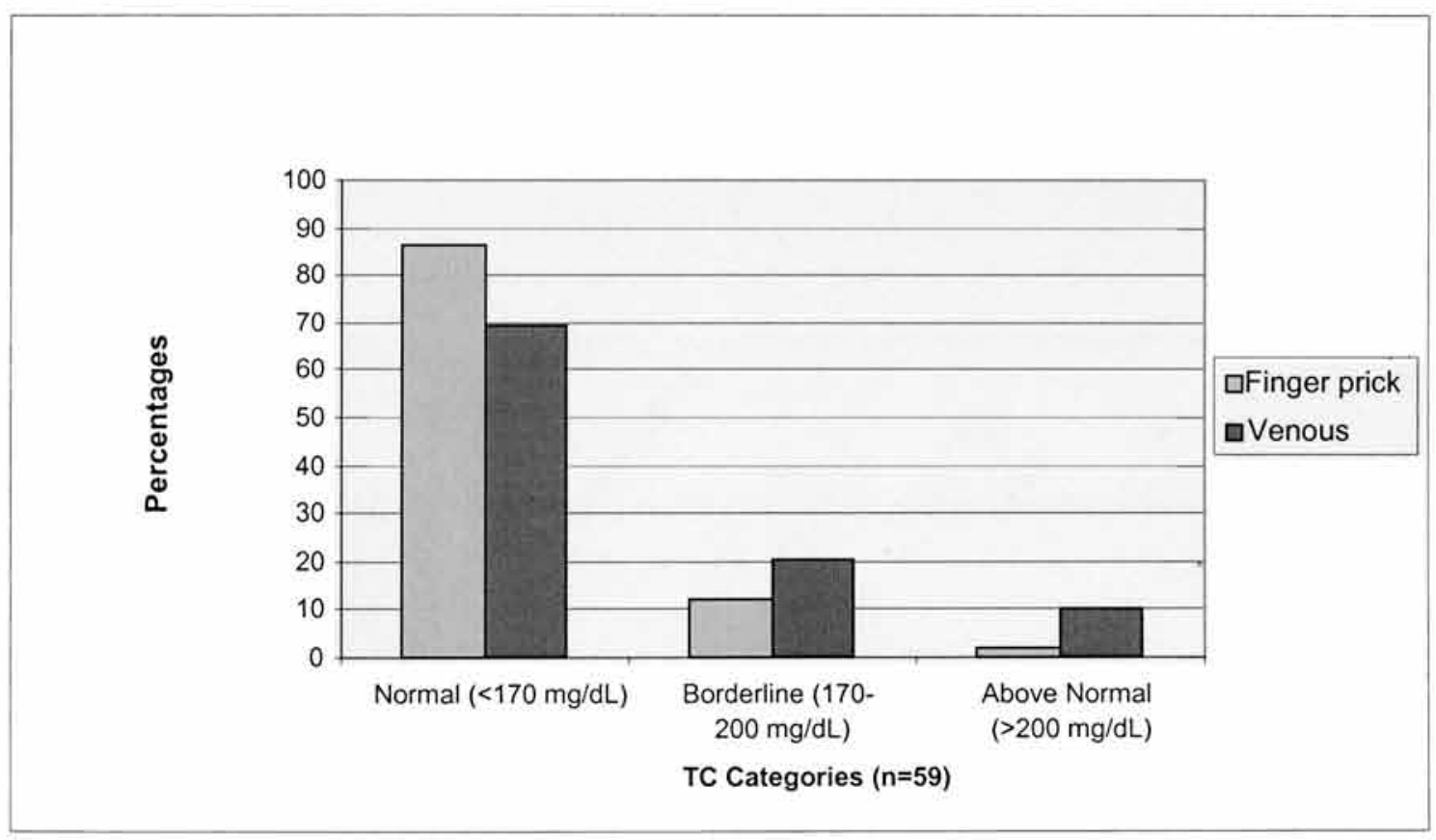

Figure 6. Comparison of Finger Prick and Venous Method TC $(\mathrm{n}=59)$

The differences between each capillary whole blood sample for TC and each corresponding venous blood sample against their means in the Bland Altman Plot 
(Figure 7) shows that the majority of the scores fell within $\pm 2 \mathrm{SD}$ which indicated good agreement between the finger prick whole blood capillary samples and venous blood samples for TC. There were no outliers for TC using box plots (Appendix X) however, when the scores were standardized for the Bland Altman Plot there were two outliers one above +3 SD and two below -2SD (Figure 7).

Since the majority of scores were a minimum of $123.52 \mathrm{mg} / \mathrm{dL}$ many of the $\mathrm{Z}$ scores fell on a straight line in the Bland Altman Plot. Correlation and Bland Altman indicated that finger prick testing of TC may be suitable for screening of risk factors for T2DM and CVDs.

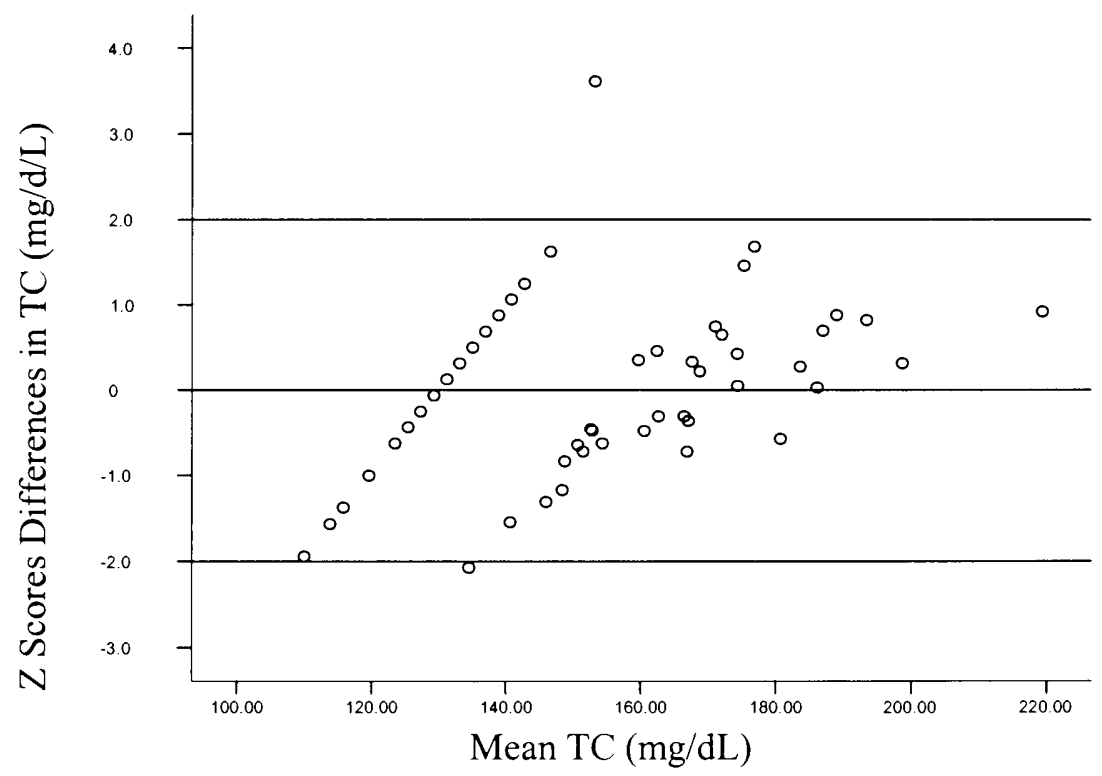

Figure 7. Bland Altman Plot for Finger Prick and Venous TC $(n=59)$ 
Comparison of Finger Prick and Venous Blood Measures for Glycated Hemoglobin $(\mathrm{HbA1c})$

$\mathrm{HbAlc}$ values for finger prick blood ranged from 4.20 to 14.0 and for venous blood 4.0 to 7.80 . Finger prick compared to venous blood testing for $\mathrm{HbAlc}$ classified more subjects at risk for T2DM than the venous blood testing $(22.0 \%$ vs. $8.5 \%)$. Comparison of $\mathrm{HbAlc}$ by both finger prick and venous blood measures (Table 22) showed a weak and insignificant correlation $(\mathrm{r}=.23, \mathrm{p}=.079)$. When further tested by paired samples t-test (Table 23), results were significantly different with a mean difference of $0.67 \pm 1.5, \mathrm{p}<.001(95 \% \mathrm{CI} 0.27-1.07)$. Figure 8 shows a comparison of $\mathrm{HbA1c}$ by the two measures. Finger prick measures of $\mathrm{HbAlc}$ revealed low sensitivity (20.0\%), and had high specificity (77.8\%) compared to venous blood testing in classifying subjects at risk for high $\mathrm{HbA1c}$.

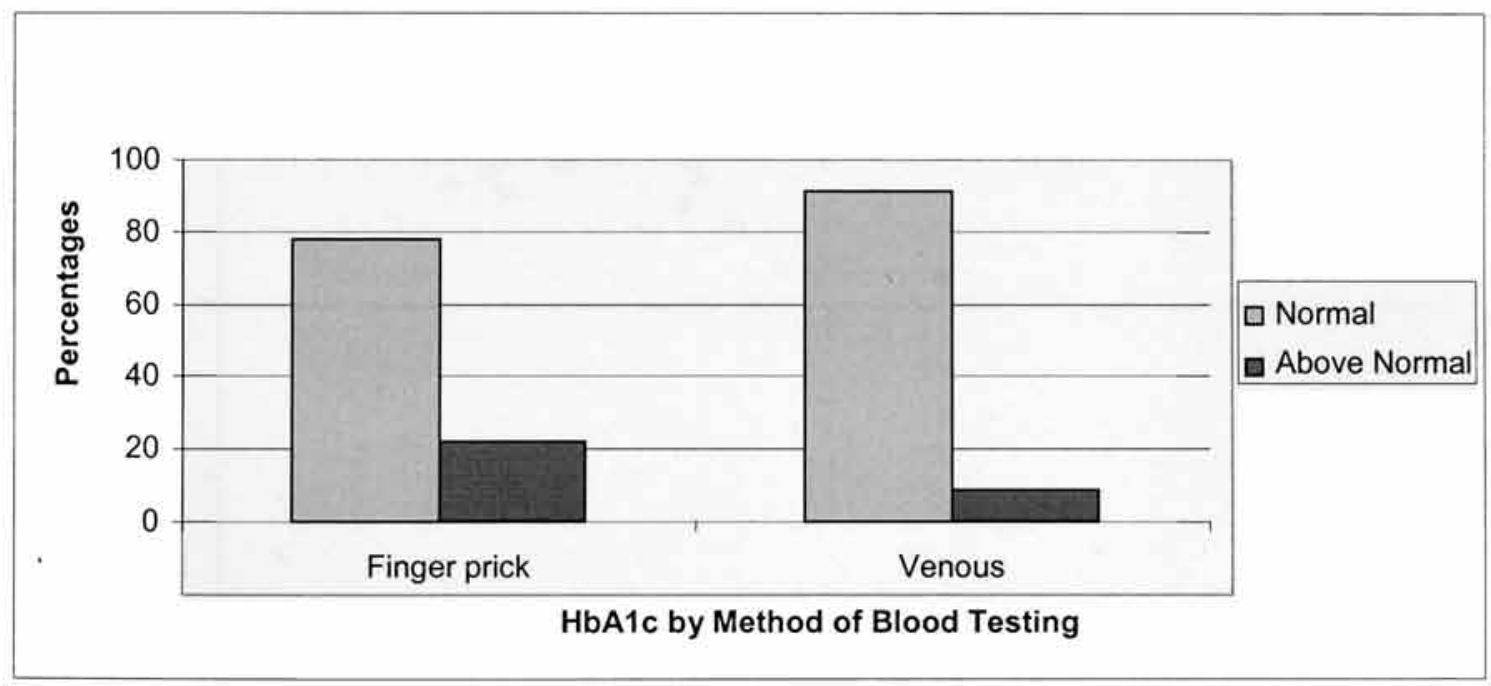

Figure 8. Comparison of Finger Prick and Venous HbAlc $(n=59)$ 
Figure 9 shows the difference between each finger prick blood sample for HbAlc and venous blood sample in a Bland Altman Plot. The majority of scores fell within $\pm 2 \mathrm{SD}$, with only two outliers for HbA1c below the -2SD in the Bland Altman Plot (Figure 9). Closer examination of the data using box plots (Appendix Y) revealed that there were many outliers for $\mathrm{HbAlc}$ for both methods, but more so for the finger prick method. Despite showing good agreement in the Bland Altman Plot between finger prick and venous blood testing of $\mathrm{HbA} 1 \mathrm{c}$, there was a weak correlation between the two measures. Similarly, paired samples t-test showed significant differences in means between the two measures. Therefore, finger prick testing of $\mathrm{HbAlc}$ may be used with caution in identifying subjects at risk for T2DM in the early screening of adolescents.



Figure 9. Bland Altman Plot for Finger Prick and Venous HbA1c $(n=59)$ 
Calculation of Percentage Bias

Comparison of finger prick testing with laboratory standards are expected to meet specific standards of variation. The percentage bias of the two methods of blood testing was calculated using the following formula;

\section{finger prick reading - laboratory value X 100 \\ laboratory value}

Glucose analysis should have analytical imprecision of $\leq 3.3 \%, \leq \pm 2.5 \%$ for bias and $\leq 7.9 \%$ for total error (Sacks, 2005). Percentage bias was $-2.4 \%$ for blood levels of 100-126 mg/dL which represented above normal levels of fasting blood glucose (FBG) and met the reference standards of $\leq \pm 2.5 \%$ for analytical imprecision (Sacks, 2005). Results for TC were compared with The National Cholesterol Education Program recommendations of $\pm 3 \%$ bias for determining total cholesterol concentration (Alberti et al., 2005; NCEP, ATPIII, 2001). Results from our study revealed percentage bias of $7.6 \%$ for TC which was below the reference standard of $\pm 3 \%$. Precision for $\mathrm{HbA} 1 \mathrm{c}$ should be $<3 \%$, and 2-3 $\%$ for established physiological limits and 3- $4 \%$ for the National Glycohemoglobin Standardization Program (Schwartz et al., 2005). Results indicated a percentage bias of $12.6 \%$ which exceeded all of the reference standards.

\section{Summary of Validation Study}

Of the three risk measures tested by finger prick testing, FBG appeared to be most suitable for the early screening of adolescents in the school setting since there was no significant difference between the finger prick and venous measures and the Bland Altman Plot showed good agreement between the means of both methods of blood 
testing. Despite the significant differences seen for finger prick and venous testing of TC and $\mathrm{HbAlc}$, the Bland Altman Plot showed good agreement for these variables which suggested they may be used with caution in the early screening of adolescents for risk factors of T2DM and CVDS in a school setting.

\section{Summary of Research Findings}

Table 24 summarized the main findings of the three hypotheses. Hypothesis one examined the relationship between the risk factors of T2DM and CVDs and the demographic characteristics of the study subjects. Hypothesis two investigated the frequency and cluster of the risk factors. Hypothesis three validated the methods of blood testing. 
Table 24

Summary of Research Findings

\begin{tabular}{|c|c|c|}
\hline Hypotheses & Findings & Interpretations \\
\hline $\begin{array}{l}\text { 1. Ethnicity, gender, place of } \\
\text { residence, and income will be } \\
\text { positively associated with the number } \\
\text { of risk factors for T2DM and CVDs } \\
\text { (high levels of FBG, HbA1c, TC, } \\
\text { BP, parents' BMI, waist } \\
\text { circumference, WHR, and presence } \\
\text { of AN, low PA, and family history of } \\
\text { CVDs, and T2DM) and with each } \\
\text { individual risk factor. }\end{array}$ & $\begin{array}{l}\text { Ethnicity significantly predicted family } \\
\text { history of } \mathrm{T} 2 \mathrm{DM}(\mathrm{OR}=2.1, \mathrm{p}=.019) \text { in } \\
\text { the logistic regression at the } .05 \text { level of } \\
\text { significance. } \\
\text { Gender significantly predicted PA } \\
(\mathrm{p}<.001) \text { in the logistic regression, and } \\
\text { total number of risk factors }(\beta=-.16, \mathrm{p} \\
=.010) \text { in the multiple regression. } \\
\text { Females were less physically active than } \\
\text { males }(\mathrm{OR}=.36, \mathrm{p}<.001) \text {. }\end{array}$ & $\begin{array}{l}\text { Hypothesis partially accepted. } \\
\text { Significance was found only for } \\
\text { gender and PA and total number of } \\
\text { risk factors after controlling for type } \\
1 \text { error using Holm's Sequential } \\
\text { Bonferroni method. } \\
\text { Females reported greater numbers of } \\
\text { risk factors than males. Females were } \\
2.7 \text { times more likely to be physically } \\
\text { inactive than males. }\end{array}$ \\
\hline $\begin{array}{l}\text { 1a. Blacks will report greater number } \\
\text { of risk factors for T2DM and CVDs } \\
\text { than Indians and those of mixed } \\
\text { ethnicities. }\end{array}$ & $\begin{array}{l}\text { Ethnicity predicted family history of } \\
\text { T2DM only }(\mathrm{OR}=2.1, \mathrm{p}=.019) \text { at the } .05 \\
\text { level of significance. }\end{array}$ & $\begin{array}{l}\text { Ethnicity was not significant for total } \\
\text { number of risk factors. Blacks } \\
\text { reported more family history of } \\
\text { T2DM. When controlled for type } 1 \\
\text { error using Holm's Sequential } \\
\text { Bonferroni method, ethnicity was no } \\
\text { longer considered significant for } \\
\text { family history of T2DM. }\end{array}$ \\
\hline
\end{tabular}




\begin{tabular}{|c|c|c|}
\hline Hypotheses & Findings & Interpretations \\
\hline $\begin{array}{l}\text { 1b. Jamaican female adolescents will } \\
\text { have a greater number of risk factors } \\
\text { for T2DM than males. }\end{array}$ & $\begin{array}{l}\text { Gender was significant for total } \\
\text { number of risk factors }(\beta=-.16, p \\
=.010) \text { at the } .05 \text { level of significance } \\
\text { in the multiple regression. }\end{array}$ & $\begin{array}{l}\text { Hypothesis accepted for gender and } \\
\text { total number of risk factors. Females } \\
\text { reported greater numbers of risk } \\
\text { factors than males. }\end{array}$ \\
\hline $\begin{array}{l}\text { 1c. Jamaican male overweight and } \\
\text { obese adolescents will have a greater } \\
\text { number of risk factors for CVDs than } \\
\text { their female obese and lean } \\
\text { counterparts. }\end{array}$ & $\begin{array}{l}\text { Gender significantly predicted FBG } \\
(\mathrm{OR}=2.01, \mathrm{p}=.040), \mathrm{WC}(\mathrm{OR}=0.56, \mathrm{p} \\
=.041), \mathrm{AN}(\mathrm{OR}=0.53, \mathrm{p}=.013), \mathrm{PA} \\
(\mathrm{OR}=.36, \mathrm{p}<.001), \text { and total number } \\
\text { of risk factors }(\beta=-.16, \mathrm{p}=.010) \text { at the } \\
.05 \text { level of significance. }\end{array}$ & $\begin{array}{l}\text { Hypothesis partially accepted. Males } \\
\text { had higher FBG than females but } \\
\text { females had higher WC, higher } \\
\text { numbers of risk factors, presence of } \\
\text { AN and were less physically active } \\
\text { than males. }\end{array}$ \\
\hline $\begin{array}{l}\text { 1d. Jamaican adolescents who live in } \\
\text { urban areas will have greater number } \\
\text { of risk factors for T2DM and CVDs } \\
\text { than their rural counterparts. }\end{array}$ & $\begin{array}{l}\text { Place of residence significantly } \\
\text { predicted } \mathrm{BMI}(\mathrm{OR}=1.87, \mathrm{p}=.018), \\
\mathrm{WC}(\mathrm{OR}=1.9, \mathrm{p}=.021), \mathrm{PA}(\mathrm{OR}=.88, \\
\mathrm{p}=.032), \text { and family history of obesity } \\
(\mathrm{OR}=0.54, \mathrm{p}=.019) \text { at the } .05 \text { level of } \\
\text { significance. }\end{array}$ & $\begin{array}{l}\text { Hypothesis not accepted after } \\
\text { controlling for type } 1 \text { error using } \\
\text { Holm's Sequential Bonferroni method. } \\
\text { Urban and rural subjects are equally } \\
\text { subject to the risk factors for T2DM } \\
\text { and CVDs. }\end{array}$ \\
\hline $\begin{array}{l}\text { 1e. Jamaican adolescents from lower } \\
\text { income families will present with } \\
\text { more risk factors for T2DM and } \\
\text { CVDs than those from higher income } \\
\text { families. }\end{array}$ & $\begin{array}{l}\text { Income significantly predicted TC } \\
(\mathrm{OR}=3.7, \mathrm{p}=.039), \text { family history of } \\
\mathrm{CVDs}(\mathrm{OR}=0.51, \mathrm{p}=.016), \text { and family } \\
\text { history of obesity }(\mathrm{OR}=0.49, \mathrm{p}=.014) \\
\text { at the } .05 \text { level of significance. }\end{array}$ & $\begin{array}{l}\text { Hypothesis not accepted after } \\
\text { controlling for type } 1 \text { error using } \\
\text { Holm's Sequential Bonferroni method. } \\
\text { Jamaican adolescents are at risk } \\
\text { forT2DM and CVDs regardless of } \\
\text { family income levels. }\end{array}$ \\
\hline
\end{tabular}




\begin{tabular}{|c|c|c|}
\hline Hypotheses & Findings & Interpretations \\
\hline $\begin{array}{l}\text { 2. Overweight and obese Jamaican } \\
\text { adolescents will have high levels of at } \\
\text { least one of the following risk factors: } \\
\text { FBG, HbA1c, TC, BP, waist } \\
\text { circumference, WHR, and presence of } \\
\text { AN, low PA, and family history of } \\
\text { overweight, CVDs, and T2DM. }\end{array}$ & $\begin{array}{l}\text { Risk factors ranged from } 0-10 \\
(3.8 \pm 2.3) . \text { Overall, } 66.7 \% \text { of normal } \\
\text { and overweight subjects had } \geq 3 \text { risk } \\
\text { factors. } 98 \% \text { of overweight and obese } \\
\text { adolescents had } \geq 3 \text { risk factors. } \\
\text { High BMI significantly increased the } \\
\text { risks of: AN }(\mathrm{OR}=22.8, \mathrm{p}<.001) \text {, } \\
\text { WHR }(\mathrm{OR}=9.1, \mathrm{p}<.001), \mathrm{TC}(\mathrm{OR}= \\
5.30, \mathrm{p}=.009), \text { family history of } \\
\text { T2DM }(\mathrm{OR}=3.70, \mathrm{p}=.001) \text {, and } \mathrm{WC} \\
(\mathrm{OR}=53.2, \mathrm{p}<.001) \text {. }\end{array}$ & $\begin{array}{l}\text { Hypothesis accepted. } \\
\text { Overweight and obese adolescents had } \\
\text { more risk factors for T2DM compared } \\
\text { to normal weight counterparts. } \\
\text { Adolescents with high BMI were more } \\
\text { likely to have presence of AN, high } \\
\text { WHR, high WC, high TC, and family } \\
\text { history of T2DM. }\end{array}$ \\
\hline $\begin{array}{l}\text { 2a.Waist circumference and WHR for } \\
\text { Jamaican adolescents will be } \\
\text { positively associated with the risk } \\
\text { factors for T2DM and CVDs (high } \\
\text { FBG, HbA1c, TC, BP, subjects' BMI, } \\
\text { and presence of AN, and low PA, and } \\
\text { family history of overweight, CVDs, } \\
\text { and T2DM, and with each individual } \\
\text { risk factor. }\end{array}$ & $\begin{array}{l}\text { WC identified more subjects at risk of } \\
\text { T2DM and CVDs }(28.6 \%) \text { compared } \\
\text { to WHR }(7.6 \%) . \text { WC positively } \\
\text { correlated } \mathrm{w} / \mathrm{BMI}(\mathrm{r}=.77, \mathrm{p}=.001), \\
\text { total number of risk factors }(\mathrm{r}=.66, \mathrm{p} \\
=.001), \mathrm{AN}(\mathrm{r}=.59, \mathrm{p}<.001), \mathrm{BP}(\mathrm{r} \\
=.39, \mathrm{p}=.001), \mathrm{FBG}(\mathrm{r}=.17, \mathrm{p}=.004), \\
\text { and family history of obesity }(\mathrm{r}=.16, \mathrm{p} \\
=.008) . \text { WHR positively associated w/ } \\
\text { total number of risk factors } \\
(\mathrm{r}=.16, \mathrm{p}=.008) \mathrm{BMI}(\mathrm{r}=.16, \mathrm{p} \\
=.009) \text { and FBG }(\mathrm{r}=.15, \mathrm{p}=.011) .\end{array}$ & $\begin{array}{l}\text { Hypothesis accepted. WC and WHR } \\
\text { positively correlated with the } \\
\text { individual risk factors and total } \\
\text { number of risk factors. } \\
\text { Subjects with high WC also had } \\
\text { higher numbers of risk factors } \\
\text { compared to those with high WHR, } \\
\text { when tested by Pearson Correlations. }\end{array}$ \\
\hline
\end{tabular}




\begin{tabular}{|c|c|c|}
\hline Hypotheses & Findings & Interpretations \\
\hline $\begin{array}{l}\text { 2b. Jamaican adolescents with family } \\
\text { history of obesity (parents' BMI) will } \\
\text { present with }>\text { BMI than those without } \\
\text { family history of obesity as } \\
\text { determined by anthropometric } \\
\text { measures of adolescents and self- } \\
\text { reported data of parents/family. }\end{array}$ & $\begin{array}{l}66 \% \text { of parents were overweight } \\
\text { /obese. Correlation found } \mathrm{b} / \mathrm{w} \text { father } \\
\& \text { subjects BMI }(\mathrm{r}=.19, \mathrm{p}<.01) \\
\text { mother and subject }(\mathrm{r}=.15, \mathrm{p}=.020) \\
\text { There were more obese adolescents } \\
(18.1 \%) \text { than mothers }(11.6 \%) \text {, and } \\
\text { father }(11.8 \%) \text {. }\end{array}$ & $\begin{array}{l}\text { Hypothesis accepted. Family history } \\
\text { of obesity related to child's obesity - } \\
\text { subjects whose parents were } \\
\text { overweight/obese had high BMI. }\end{array}$ \\
\hline $\begin{array}{l}\text { 2c. Energy intakes of Jamaican } \\
\text { adolescents will be positively } \\
\text { associated with the total number of } \\
\text { risk factors for T2DM and CVDs and } \\
\text { with each individual risk factor. }\end{array}$ & $\begin{array}{l}\text { Macronutrient intakes were within the } \\
\text { AMDR ranges. } \\
\text { Total fat intake correlated w/ WC } \\
(\mathrm{r}=-.18, \mathrm{p}=.003) .\end{array}$ & $\begin{array}{l}\text { Hypothesis not accepted. After } \\
\text { controlling for type } 1 \text { error, } \\
\text { significance found only for WC and } \\
\text { total fat. }\end{array}$ \\
\hline $\begin{array}{l}\text { 2d. Intakes of complex and refined } \\
\text { carbohydrates of Jamaican adolescents } \\
\text { will be positively associated with the } \\
\text { total number of risk factors for T2DM } \\
\text { and CVDs (high levels of FBG, } \\
\text { HbAlc, TC, BP, waist circumference, } \\
\text { WHR, and presence of AN, low PA, } \\
\text { and family history of overweight, } \\
\text { CVDs, and T2DM) and with each } \\
\text { individual risk factor. }\end{array}$ & $\begin{array}{l}\text { Complex carbohydrates negatively } \\
\text { correlated } \mathrm{w} / \mathrm{AN}(\mathrm{r}=-.15, \mathrm{p}=.011) \text {, } \\
\text { and total number of risk factors } \\
(\mathrm{r}=-.13, \mathrm{p}=.030) \text {, and positively } \mathrm{w} / \\
\text { PA }(\mathrm{r}=.13, \mathrm{p}=.026) \text {. } \\
\text { Intakes of refined carbohydrates had } \\
\text { negative correlations } \mathrm{w} / \mathrm{BMI} \\
(\mathrm{r}=-.14, \mathrm{p}=.020) \text { and PA }(\mathrm{r}=-.13 \text {, } \\
\mathrm{p}=.029) .\end{array}$ & $\begin{array}{l}\text { Complex carbohydrates correlated w/ } \\
\text { more risk factors than refined sugars at } \\
\text { the } .05 \text { level. } \\
\text { Hypothesis not accepted. Intakes of } \\
\text { refined sugars and the risk factors } \\
\text { were not significant using Holm's } \\
\text { Sequential Bonferroni method. }\end{array}$ \\
\hline
\end{tabular}




\begin{tabular}{|c|c|c|}
\hline Hypotheses & Findings & Interpretations \\
\hline $\begin{array}{l}\text { 2e. Total fiber intake }(\mathrm{g}) \text { of Jamaican } \\
\text { adolescents will be negatively } \\
\text { associated with the total number of } \\
\text { risk factors for T2DM and CVDs and } \\
\text { with each individual risk factor. }\end{array}$ & $\begin{array}{l}\text { Fiber intakes negatively correlated } \mathrm{w} / \\
\text { BMI }(\mathrm{r}=-.15, \mathrm{p}=.015), \text { WC }(\mathrm{r}=-.15, \mathrm{p} \\
=.012), \text { family history of obesity } \\
(\mathrm{r}=-.17, \mathrm{p}=.005), \text { AN }(\mathrm{r}=-.18 \\
\mathrm{p}=.003), \text { and total number of risk } \\
\text { factors }(\mathrm{r}=-.14, \mathrm{p}=.017)\end{array}$ & $\begin{array}{l}\text { Hypothesis not accepted. Significance } \\
\text { found only for AN and fiber intake } \\
\text { using Holm's Sequential Bonferroni } \\
\text { method. High intakes of fiber were } \\
\text { associated with absence of AN. }\end{array}$ \\
\hline $\begin{array}{l}2 \mathrm{f} \text {. Intakes of saturated and } \\
\text { unsaturated fat, and intake of omega- } 3 \\
\text { and omega- } 6 \text { fatty acids of Jamaican } \\
\text { adolescents will be associated with the } \\
\text { total number of risk factors for T2DM } \\
\text { and CVDs and with each individual } \\
\text { risk factor }\end{array}$ & $\begin{array}{l}\text { Intakes of saturated fats negatively } \\
\text { correlated } \mathrm{w} / \mathrm{WC}(\mathrm{r}=-.16, \mathrm{p}=.007) \text {, } \\
\text { and } \mathrm{AN}(\mathrm{r}=-.17, \mathrm{p}=.006) . \\
\text { Monounsaturated fats were positively } \\
\text { associated } \mathrm{w} / \mathrm{HbA} 1 \mathrm{c}(\mathrm{r}=.13 \text {, } \\
\mathrm{p}=.032), \text { and negatively } \mathrm{w} / \mathrm{WC} \\
(\mathrm{r}=-.15, \mathrm{p}=.013) \text {, and AN } \\
(\mathrm{r}=-.17, \mathrm{p}=.005) . \text { Polyunsaturated } \\
\text { fats were positively associated } \mathrm{w} / \\
\text { HbAlc }(\mathrm{r}=.13, \mathrm{p}=.031) \text {, and } \\
\text { negatively with BMI }(\mathrm{r}=-.12, \mathrm{p} \\
=.043), \mathrm{WC}(\mathrm{r}=-.13, \mathrm{p}=.028) \text {, and } \\
\text { AN }(\mathrm{r}=-.15, \mathrm{p}=.011) .\end{array}$ & $\begin{array}{l}\text { Hypothesis not accepted. There were } \\
\text { no significant associations with the } \\
\text { types of fats and the risk factors of } \\
\text { T2DM and CVDs after controlling for } \\
\text { type } 1 \text { error using Holm's Sequential } \\
\text { Bonferroni method. } \\
\text { Intakes of omega- } 3 \text { and omega- } 6 \text { fatty } \\
\text { acids were negligible in this } \\
\text { population. No significant correlations } \\
\text { found between the individual risk } \\
\text { factors and omega- } 3 \text { or omega- } 6 \text { fatty } \\
\text { acids or cholesterol intakes. }\end{array}$ \\
\hline $\begin{array}{l}\text { 3. There will be no significant } \\
\text { differences between FBG, TC, and } \\
\text { HbA1c measured by finger prick and } \\
\text { venipuncture methods. }\end{array}$ & $\begin{array}{l}\text { Paired samples } t \text {-tests showed no } \\
\text { significance for Finger prick FBG and } \\
\text { venous blood FPG }(\mathrm{p}=.185) \text {, but } \\
\text { Finger prick TC and HbAlc were } \\
\text { significantly different from venous } \\
\text { blood samples }(\mathrm{p}=.001) \text {. }\end{array}$ & $\begin{array}{l}\text { Hypothesis accepted for FBG but not } \\
\text { for TC and HbAlc when tested for } \\
\text { significance. Good agreement in the } \\
\text { Bland Altman Plot indicated all three } \\
\text { tests can be used for screening of high } \\
\text { school adolescents in this sample. }\end{array}$ \\
\hline
\end{tabular}




\section{CHAPTER V}

\section{DISCUSSION}

Type 2 diabetes mellitus (T2DM) is increasing among younger populations in the US (Sacks et al., 2002; Daniels, 2006; Choudbury: 2005 \& Reinehr, 2005). This increase has been linked to the growing rates of obesity. Obesity is further linked to the development of cardiovascular diseases (CVDs). We predicted similar findings among the Jamaican adolescent population. In one nationwide survey, $25 \%$ of Jamaican adolescents were found to be overweight ("The Jamaican Daily Gleaner", 2007). Despite the growing rates of obesity and T2DM among adolescents in other countries, research is lacking on Jamaican adolescents who are expected to share similar characteristics. Additionally, there is no documented study on risk factors of T2DM among Jamaican adolescents. Our study examined if Jamaican adolescents are at risk for T2DM and CVDs as found in similar populations in the resource-rich countries. Socio-demographic characteristics of this population and the prevalence of 13 risk factors for T2DM and CVDs were described and their associations were analyzed.

\section{General Description of the Study Subjects}

The recruitment and sampling process successfully obtained sufficient numbers of subjects to fit the selection criteria set in the study for place of residence (rural, urban), gender and socio-economic status (SES). We were unable to recruit sufficient numbers from the different ethnic groups due to the representative random selection process that was followed. A quota non-probability sampling method would have ensured adequate sample size from all other ethnically diverse groups but would not have allowed for 
randomization and for our sample to be representative of the general population. The use of schools as the research site allowed for easy access to study subjects. Moreover, data collection on all thirteen risk factors was completed in one day at each of the ten schools. The easy access to study subjects and methods of data collection also allowed completion of pilot testing of the instruments, recruitment, and data collection in one year.

Gender was important to our study since earlier research showed that females are more likely to have T2DM and males tend to be more at risk for CVDs. Our final sample included more females than males. This discrepancy occurred among the higher grade levels (11-12) with more females (60) than males (43) and also by the "no shows" on data collection days. In the upper grade levels more females tend to dominate since this is the time the students prepare for the advanced level subjects for external examinations and for entry to tertiary institutions. Typically more females than males would stay on for further schooling at this level. This trend continues into tertiary level with more females than males attending university (Miller, 1986). Students were needed from these upper grades to include the age group 18-19 years since our study dictated inclusion of subjects ages $14-19$ years.

Gender and ethnicity were considered significant to this study since research has shown gender and ethnic differences for many of the risk factors for T2DM and CVDs. For example, in previous studies, more adolescent females than males were obese and adolescent females were 1.7 times more likely to develop T2DM than males (Rosenbloom, et al, 1999; McKnight-Menci et al, 2005). Our study found only 3 subjects with fasting blood glucose (FBG) values $>126 \mathrm{mg} / \mathrm{dL}$ which suggested the presence of diabetes. These students were referred for further follow-up. Our one-time measure of 
fasting blood glucose is not conclusive for diagnosing diabetes among our study sample. Unlike earlier studies which showed more adolescent girls than boys to be at risk for T2DM, adolescent boys were two times more likely to have high FBG compared to adolescent girls in our study. Gaskin \& Walker (2003) found significantly higher $(\mathrm{p}<.01)$ BMI among Jamaican girls $\left(18.01 \pm 3.34 \mathrm{~kg} / \mathrm{m}^{2}\right)$ than boys $\left(17.15 \pm 2.55 \mathrm{~kg} / \mathrm{m}^{2}\right)$ ages 10-12 years. We found slightly higher BMI for females $(24.4 \pm 8.2)$ compared to males $(22.76 \pm 6.8)$ in our study but this was not statistically significant. Research also indicated that adolescent males tend to have more abdominal fat than total fat mass. Abdominal fat correlated better with the metabolic risk factors of CVDs than total fat (Moreno, Pineda, \& Rodriguez et al., 2002). In another study, adult males were shown to be more at risk for CVDs than females (England Health Survey for Ethnic Minority Groups, 1999). Fat cells associated with obesity resists insulin therefore sugar tends to remain elevated in the blood. Weight reduction and consequent lowering of BMI improves insulin sensitivity and may prevent or delay the onset of T2DM in at-risk adolescents.

More Blacks participated in this study than other ethnic groups present which was expected since up to $91 \%$ of the island's population is Black. Research has shown that globally the highest rate of T2DM is predicted to come from India (WHO, 2003 \& Singh et al, 2004). However, in this study only $3.9 \%$ of the sample was Indians, consistent with their representation in the general population. The majority of the Indians were females aged 14-16 years and were drawn from the rural schools. The mixed ethnic group comprised $17 \%$ of the sample and was predominantly from the traditional high schools. 
There was an almost even distribution of this mixed group among urban and rural locations and were from the age groups 14-18 years.

It was not surprising to find a higher percentage of the "mixed ethnicity" in our sample than the Indians since overall, the islands' population comprises $7 \%$ of the mixed ethnicities compared to $1 \%$ Indians (Statistical Institute of Jamaica, Census, 2000) and there are a number of inter-racial marriages among Blacks and Indians. In some cases, mixed group represented more than two ethnic groups; some of the subjects reported being from Black, Indian, and Caucasian ethnicities. For analyses purposes, ethnicity was grouped as Blacks and Non- Blacks: Indians, Chinese, Caucasian and those of mixed ethnicities.

School-type was used to represent socio economic status (SES) since the traditional schools typically cater to the upper and middle class students. There were few more subjects from the non-traditional compared to traditional high schools. This difference can be accounted for by the number of no-shows since the four subjects who failed to return the consent forms were from a traditional high school. One traditional high school accounted for eight ( 4 did not return the signed parental consent form, and 4 were absent on the day of data collection) out of the twenty-four no shows. The three study subjects who were excluded from the study for incomplete data were also from a traditional high school.

The majority of the sample fell in the 16 year old age group. Although equal numbers of subjects were sought by place of residence (rural, urban) more urban than rural subjects participated in the study, again, a consequence of the representation of the sample. Lower participation was a result of the heavy rains which prevented rural 
students from attending school on data collection day. Our sample was representative of Jamaican adolescents with predominantly more Blacks and females, almost equal numbers of rural and urban adolescents and more subjects from the non-traditional high schools.

\section{Anthropometric Measures}

Body mass index (BMI) and other anthropometric measures were of interest to our study since research shows that individuals with higher BMI, WC and WHR are at risk for T2DM and CVDs. Determining BMI was important for our study since previous studies from large population groups indicated that obesity increased the risk of mortality from CVDs. Subjects with BMI $>35 \mathrm{~kg} / \mathrm{m}^{2}$ are 2-3 times more likely than lean persons with lower BMI (18.5- $24.9 \mathrm{~kg} / \mathrm{m}^{2}$ ) to die from CVDs (Calderon et al, 2004).

Overweight in adolescent is associated with a $130 \%$ increased risk of CVDs mortality during adulthood (Calderon et al, 2004). Still, other studies show that increased BMI is a risk factor for T2DM (Reinher, 2005, \& Steinberger et al., 2001). In another study, overweight in children and adolescents strongly predicted increased risks of morbidity and mortality in adulthood regardless of their adult weight status (Deckelbaum \& Williams, 2001).

Our findings indicated that overweight in Jamaican adolescents was twice the prevalence rate of overweight adolescents in the USA. The prevalence rate of obesity (BMI exceeding the $95^{\text {th }}$ percentile) among US children aged 12-19 years was estimated at $15.5 \%$ in 2000 (Bloomgarden, 2004). We found that $33 \%$ of our sample was overweight by the same standard. The percentage of overweight adolescents in the 
present study was above the estimated $25 \%$ of the Jamaican Ministry of Health Report (“Jamaican Daily Gleaner," 2007). These results indicated a growing percentage of adolescents becoming overweight. There is a need for preventive programs which target weight control. These programs can be implemented within the Jamaican school system.

Almost $25 \%$ of our sample was classified as underweight. This finding on underweight is similar to earlier research which indicated that over-nutrition and undernutrition go hand-in-hand in some resource-poor countries (Popkins, 2001; Prentice, 2006). It was not surprising to find underweight children and adolescents with their overweight mothers in the developing world. The presence of more overweight mothers may be due to more domestic lifestyles of women compared to children who are engaged in more activities outside of the home. Typically, women in the developing world are responsible for preparing the family's meals which gives them more access to food than the rest of the family. It is likely that non-working mothers would be more overweight than their children. Further analysis of employment status of mothers and overweight is suggested. These findings are important to Jamaican health workers who can target programs for adolescents. Further research is needed on the dietary and other factors which contribute to the underweight among the Jamaican adolescents.

More females than males had BMI in the overweight range $\left(\mathrm{BMI}>25 \mathrm{~kg} / \mathrm{m}^{2}\right)$. There were higher percentages of subjects ages 14-16 with BMI in the overweight range compared to the $17-19$ year olds $(11.6 \%$ vs. $2.9 \%)$ respectively. This may be related to pubertal development. However, we did not measure pubertal stages. Nevertheless, our results on the prevalence of overweight among younger adolescents indicated that measures are needed to stop the spread of this health condition which will lead to T2DM 
and CVDs in the later years. It further supports the need for early screening for risk factors of T2DM and CVDs among high school students.

Results of our study on BMI, gender, and age are similar to previous findings of Gaskin \& Walker (2003). Jamaican girls had significantly higher $(\mathrm{p}<.01)$ BMI compared to boys in the 10-12 year group. Similarly, another study found more obese Jamaican adult females compared to males (Ichinohe, et al., 2005). As supported by other literature (Popkins, 2001; Prentice, 2006), a higher percentage of urban compared to rural adolescents were overweight in our study. Earlier research shows a shift towards the consumption of more high-fat and high-calorie foods, and decreased physical activity among urban than rural children. These findings are crucial to health administrators who can plan and implement preventive programs to target weight control among Jamaican adolescents.

Physical Activity

Earlier research showed that decreased physical activity (PA) and time spent watching television were linked to childhood obesity (Crespo et al, 2002; Certain et al, 2001; Dennison et al, 2002; \& Christakis et al, 2004). In the current study, subjects spent approximately 10 hours per week watching television or an average of 1.4 hours per day and up to 5.9 hours per week playing computer games. Research is lacking on just how much physical activity is needed among children and adolescents to prevent obesity. However, others have found that children are not sufficiently physically active (Crespo et al, 2002; Dennison et al, 2002; Dowda et al, 2001 \& Gortmaker et al, 1996). Approximately 30 minutes of physical activity per day is recommended by the US 
Surgeon General for people over two years (Welk \& Wood, 2000). A limitation of our study was that the Physical Activity Questionnaire used for Children (PAQ-C) did not measure the amount of time spent on each activity but gave an indication of how often and what types of activities they were more likely to be engaged in. However, despite this limitation, data on frequency and types of activities may be used to plan intervention programs to increase PA among adolescents.

A number of studies have shown the benefits of PA in the prevention of T2DM, CVDs, and hypertension (Pan, Li, \& Hu, 1997; Leon, 1997; \& Tuomilehto, Lindstrom, \& Erickson et al., 2001). Results from these earlier studies indicated that inactivity was closely related to chronic non-communicable diseases. Physical activity was measured in the current study since it was expected that low PA levels would be related to risk factors associated with T2DM and CVDs. PA was found to be significant only for gender in this study when tested by logistic regression. Males were more physically active compared to females. Adolescent males may have more leisure time compared to females. It is part of the Jamaican culture to assign more household chores to the females leaving them with less time for the kinds of physical activities measured by the PAQ-C. Although data are lacking on PA and risk of T2DM and CVDs among Jamaican adolescents, previous research on Jamaican adults showed a similar trend with more females than males being physically inactive (Ichinohe, et al., 2005). Early intervention is needed to target more involvement in PA among Jamaican adolescent girls since there is research evidence of more Jamaican adult females being physically inactive.

It was expected that PA would predict BMI. However, PA was not a significant factor for BMI in our study $(\mathrm{p}=.850)$. The majority of the subjects reported PA more than 
two times per week which classified them as being physically active. Also, the kinds of activities listed on the scale are typical activities for Jamaican adolescents. These activities varied from skipping, walking, riding and bicycling which are common activities especially among rural Jamaican children. Therefore we expected more rural than urban adolescents to be physically active. A better measure of the relation between PA and BMI would have been to determine the amount of time and energy spent on each activity. Despite some significance found for PA and risk factors at the .05 level of significance, when we controlled for type 1 error, significance was found only between PA and gender. Nevertheless, findings on PA from this study are significant since it provides preliminary data for further comparisons.

Our findings on PA are supported and refuted by other studies. Earlier research found a positive correlation between being female, urban resident and distance walked or cycled to school each day (Sjolie and Thuen, 2002). Border, Rohrer, \& Cardarelli (2006) found significant differences between PA and place of residence with adult males and females in non-metropolitan (rural) areas having higher odds of obesity than their metropolitan (urban) counterparts. These studies were conducted on an older population than ours. It is expected that with low PA, female adolescents in our study also face the risk of T2DM and CVDs in later years. More research is needed on physical activity and risk factors of T2DM and CVDs of Jamaican adolescents and lifestyle factors that may lend itself to increased risk of chronic diseases. 
This study was unprecedented in the examination of Jamaican adolescents for the presence of AN. The presence of AN was easily determined by the dark line around the neck, axilla, knees, elbows (Scott et al., 1997). For statistical analysis, presence of AN was determined by the neck measures only in our study. Over forty percent of our sample had AN on the neck area. There was no conclusive evidence that these subjects had T2DM, but the presence of $\mathrm{AN}$ is considered a risk factor for T2DM perhaps in later years as indicative of metabolic syndrome (Kong, Williams, \& Smith et al., 2007). The ease and low cost of screening for $\mathrm{AN}$ are helpful in identifying at risk populations and in planning programs for the prevention of T2DM.

Previous research have found that the presence of AN is an independent risk factor for T2DM since AN is associated with hyperinsulinemia and high BMI (Kong, et al., 2007; Kobaissi, Weigensberg, Ball et al, 2004). One third of our sample had high BMI. The presence of $\mathrm{AN}$ in our study was strongly related to high BMI which is also a risk factor for T2DM and CVDs. Subjects with AN were 28 times more likely to have high BMI than those without AN. We further found a strong correlation between the presence of AN and BMI ( $r=.633, p=.001)$. Unlike other studies (Kong et al., $2007 \&$ Kobaisi et al., 2004) we did not measure hyperinsuleinemia. Hyperinsulinemia results in insulin resistance (IR) and is related to high BMI. The one-time only finger prick method of testing FBG used in this study could not allow for us to measure IR. Insulin resistance is commonly measured by homeostatic model assessment (HOMA) which uses fasting plasma glucose and insulin concentrations to determine indices of insulin sensitivity and secretion (Tripathy, Almgren, \& Tuomi et al, 2004). 
The presence of AN is closely related to high BMI and other risk factors of

T2DM. A more recent study (Kong et al, 2007) examined 1133 US children ages 7-19 for AN and the risk factors for T2DM such as family history of T2DM, hypertension, and overweight/obesity. They found that AN was present in $17 \%$ of the children and adolescent in their sample. Another study of 131 obese children (BMI of 28.4 \pm 5.5 ) ages 7-13 found that up to $73 \%$ of the children had AN (Kobaissi, et al, 2004). Findings of overweight associated with $\mathrm{AN}$ in children and adolescents are significant. Acanthosis Nigricans is easy to detect and this can guide programs for targeting at risk adolescents to prevent T2DM and CVDs at an earlier age before symptoms develop, thereby reducing the rates of morbidity and mortality in later years.

The association between AN, ethnicity and T2DM was strong in other studies (Fagot-Campagna et al., 2000; \& Stuart et al., 1998). The presence of AN is a physical marker for insulin resistance (IR) which is common among minority ethnic groups in the USA. Up to $40 \%$ of Native Americans, $13 \%$ of African Americans and 6\% of Hispanics with IR also had AN (Tajima, 2002; Fagot-Campagna et al.2000; \& Stuart et al, 1998). In the absence of IR, BMI remains a strong indicator of AN. Three studies (Nguyen et al., 2001; Hirschler, et al., 2002 \& Kobaissi, et al., 2004) found closer relationships between AN and BMI when compared to AN and hyperinsulinemia and IR. Only $35 \%$ of the obese children with hyperinsulinemia also had AN despite ethnicity (Nguyen et al., 2001). Kobaissi, et al. (2004) found that BMI was a better indicator of AN than IR among Hispanic children and adolescents. Therefore we consider our findings on the relationship between BMI and AN to be a strong indicator of the risk of T2DM among the population we studied. 
Overall, our study found $44 \%$ of the subjects had AN, $15 \%$ had elevated levels of FBG, and $33 \%$ were found to be overweight which are strong indicators for the development of T2DM and CVDs in later years. Kobaissi et al. (2004) found higher presence of AN (73\%) compared to our study (44\%) however, in their study subjects had higher mean BMI than in our study. Although AN was significantly correlated with some variables (WC, WHR, and fiber intake) in this study, the presence of AN alone was not conclusive for predicting the presence of diabetes since IR was not measured. Kong et al (2007) suggested that AN is an independent risk factor for T2DM and that individuals with $\mathrm{AN}$ are likely to present with multiple risk factors for T2DM. These data can be used to guide further research on the presence of AN and the specific risk factors for T2DM among Jamaican adolescents.

Socio-Demographic Characteristics and Risk Factors of T2DM and CVDs

We hypothesized that ethnicity (Blacks, non-Blacks), gender (male, female), place of residence (rural, urban), and income (high, low) would be positively associated with the risk factors for T2DM and CVDs based on earlier research findings (WHO, 2003; Singh et al, 2004; Rosenbloom, 1999; McKnight-Menci et al, 2005 \& Moreno et al., 2002). Ethnicity was grouped as Blacks and non-Blacks in our study. The latter included Indians, Chinese, Caucasian, and subjects of mixed ethnicities. Gender, ethnicity, place of residence, and income predicted some of the risk factors of T2DM and CVDs in the logistic regression at the .05 level of significance. However, after controlling for type 1 error, we found significance only for gender and PA. Males were more physically active than females in this study. 
We hypothesized that females would have greater number of risk factors for T2DM than males since other studies indicated that female adolescents were 1.7 times more likely to develop T2DM than males (Rosenbloom, et al., 1999; McKnight-Menci et al., 2005). Our hypothesis was partially accepted since gender significantly predicted only PA in the logistic regression with adolescent males being more physically active than females. However, females had significantly more risk factors than males in the multiple regression. Our findings on gender and number of risk factors are similar to earlier studies on gender and risk of T2DM (Rosenbloom, et al., 1999, McKnight-Menci et al., 2005).

We hypothesized that Black Jamaican adolescents would have greater number of risk factors for T2DM and CVDs than Indians and those of mixed ethnicities since results from US based studies indicated that more Blacks than Whites were at risk for T2DM (Sinha, et al., 2002; ADA, 2000; ADA, 2005; Bavdekar et al, 1999; Berry et al., 2004; \& Kimm, et al., 2001). When controlled for type 1 error, ethnicity did not predict any of the risk factors in the logistic regression. However, cross-tabulation of the risk factors related to the METS (HbAlc, FBG, TC, WC and BP) among ethnic groups indicated that a higher percentage of non-Blacks classified with the METS (had $\geq 3$ risk factors) compared to Blacks in our study.

We predicted that place of residence (urban, rural) would be significantly related to the risk factors of T2DM and CVDs. Specifically, more urban adolescents would have greater number of risk factors for T2DM and CVDs than their rural counterparts due to their decreased PA levels, and higher intakes of energy-dense foods (Popkins, 2001; 
Prentice, 2005). However, we found no significant association with place of residence and the risk factors for T2DM and CVDs after controlling for type 1 error.

It was hypothesized that subjects from the lower income group would have greater number of risk factors for T2DM and CVDs as reported by other studies (Drewnoski \& Darmon, 2005a; Drewnoski \& Darmon, 2005b; Drewnowski, 2007; Monteira, et al., 2004; Blakely, Hales, Kieft et al., 2004; Cooper, Cutler, Desvigne et al., 2000). Income was a significant predictor of TC, family history of obesity, and family history of CVDs at the .05 level of significance in our study but not after controlling for type 1 error. Contrary to expectations, higher TC levels were found in the low income group compared to the high income group which was expected to consume greater amounts of animal foods.

Higher rates of obesity have been found in low-income groups in US based studies (Drewnoski \& Darmon, 2005a; Drewnoski \& Darmon, 2005b; Drewnowski, 2007). However, we found that subjects from high income group were more likely to report family history of obesity than those from low income group. Similarly, subjects from the high income group reported more family members with family history of CVDs than those from the low income group. Earlier research (Drewnoski, 2007) indicated that the taste and low cost of high fat foods and refined sugars make them more appealing and economical to low income groups.

The lack of significance between income and the risk factors among our study population may be the result of the high cost of foods. Foods that are considered low cost in the US are not the same as in the Jamaican diet. Most of the US low-cost foods when exported to Jamaica become high-cost due to the devaluation of the Jamaican dollar. It is 
likely that these foods are affordable mainly to the high income group. The high costs of these foods make them more appealing to individuals with higher incomes, regardless of the association with chronic diseases.

Family history of CVDs was determined if the parent(s) or other family members had been diagnosed by a nurse or doctor for having angina or stroke. Based on these criteria, more family history of CVDs was found among the high income group compared to the low income group. This was not surprising since the high income group reported more family history of obesity which is a risk factor for CVDs. Earlier literature referred to CVD as a "disease of affluence" or "Western disease" since it was mainly found among the high income group (Mckeown, 1988; Trowell \& Burkett, 1981). Our findings of significantly higher percentage of family history of obesity and CVDs among the high income group appeared to fit this paradigm of disease of affluence. Nevertheless, other studies have shown that among upper-middle income, and high income countries, CVDs risk factors are found mainly among the lowest-income group (Monteira, et al., 2004; Blakely, et al., 2004; Cooper, et al., 2000).

Our findings on income and the risk factors of T2DM and CVDs are significant. Further research is needed on income, food costs, food intakes and relation to T2DM and CVDs of our study population. The findings of effect of gender, ethnicity, place of residence, and income on the risk factors of T2DM and CVDs were confounded by the numbers of risk factors examined. 
The main aim of our study was to determine if Jamaican adolescents were at risk for T2DM and CVDs as observed in similar populations in other countries. We coded twelve risk factors (FBG, TC, HbAlc, BP, PA, AN, WC, WHR, BMI, family history of T2DM, CVDs and obesity) as present or absent based on current cut-off criteria as described in the methods section. Over two-thirds of our population reported presence of more than three risk factors. However, these risk factors did not relate specifically to the presence of the metabolic syndrome (METS). Therefore we further selected five risk factors which more closely matched the presence of the metabolic syndrome [FBG, TC, $\mathrm{HbA1c}, \mathrm{BP}$ and central adiposity (high waist circumference)]. We found that $13.7 \%$ of the sample presented with three or more of these five selected risk factors. Presence of three or more of these risk factors indicated presence of the METS.

Our five selected risk factors for the METS were based on different criteria and included the suggestions of the WHO's Diabetes Group, and The National Cholesterol Education Program (NCEP) Adult Treatment Panel III in the USA (2006). The WHO Diabetes Group suggested the use of impaired glucose tolerance (IGT) or diabetes, with at least two other risk factors from the following list: raised blood pressure, hypertriglyceridaemia and or low HDL-C, obesity measured by high WHR or high BMI, and microalbuminuria (Alberti \& Zimmet, \& Shaw 1998). We did not use WHO criteria since we did not measure IGT, and HDL-C. Therefore, we selected risk factors based on The National Cholesterol Education Program (NCEP) Adult Treatment Panel III in the USA (2006) which stated that the presence of any three of the five components of CVDs risk to include central obesity, raised blood pressure, raised triglycerides, low HDL-C, 
and fasting hyperglycemia constitute the METS. The METS criteria of the NCEP were closer to our measures of risk factors of T2DM and CVDs. In the present study, high FBG and $\mathrm{HbAlc}$ were considered as surrogates for hyperglycemia and subsequent risk for T2DM.

Although in this study IR was not measured, we considered our findings on the presence of the METS adequate. Earlier research pointed to insulin resistance as a causative factor in the METS (Reaven, 1988). However, the role of IR in the METS is still controversial (Alberti, et al., 2005). The International Diabetes Federation suggested that in clinical practice, the presence of impaired glucose is applicable, but all reports on the prevalence of the METS should use only fasting plasma glucose (FPG) of previously diagnosed diabetes when defining hyperglyceridemia (Alberti, et al., 2005). A limitation of our study is that fasting plasma glucose (FPG) was measured only on our sub-study sample of 59 subjects while FBG was used for all 276 participants. As indicated in the results section fasting plasma glucose for the sub-study sample underestimated the number of subjects with normal FPG and overestimated those with high IFG. Further, glucose concentrations in plasma are lower than whole blood since the concentration of water is approximately $11 \%$ higher in plasma than the whole blood (Sacks et al., 2002). Fasting blood glucose is likely to overestimate glucose concentrations and is not as reliable as FPG values (Sacks et al., 2002). Finger prick FBG are therefore suitable for screening to be followed by venous FPG testing for diagnosis.

Elevated triglycerides (TG) or the lipid triad which includes high TG, low HDL-C and high LDL-C are indicators of atherogenic dyslipidemia (Li \& Ford, 2007). Research indicated that TG appeared to be better indicator of dyslipidemia in defining the 
metabolic syndrome in adolescents than the lipid triad (Li \& Ford, 2007). A limitation of the present study was that TG was not measured using finger prick method. A complete lipid profile was assessed for the 59 subjects that comprised the sub-study sample and none of these subjects had triglyceride levels $>200 \mathrm{mg} / \mathrm{dL}$. It is likely that the main sample would not have presented with high TG levels since we randomly selected our sub-study sample from the main study sample and paired comparisons were made.

Previous research has shown that up to $60 \%$ of overweight children and adolescents had at least one cardiovascular risk factor such as high cholesterol, elevated insulin, and hypertension compared to $10 \%$ of those with healthy weight who had at least one risk factor for T2DM and CVDs (Freedman, et al, 1999). Freedman et al (1999) also found that up to $25 \%$ of overweight adolescents had two or more risk factors. In general, over two thirds of our sample, overweight or not, had three or more risk factors of T2DM and CVDs.

We further compared risk factors for overweight and underweight/normal weight subjects and found that $98 \%$ of our overweight subjects had more than 3 risk factors for T2DM and CVDS compared to $46 \%$ for the underweight and normal weight subjects. The number of subjects with 5 or more risk factors greatly increased for subjects with BMI $\geq 25 \mathrm{~kg} / \mathrm{m}^{2}$ compared to those with BMI $<25 \mathrm{~kg} / \mathrm{m}^{2}$. Our findings on overweight and number of risk factors in adolescents are higher compared to US based studies. Jamaican adolescents may be at higher risk for chronic diseases as compared to their US counterparts.

Risk factors tend to cluster and the presence of more than one risk factor in childhood increases the risk of CVDs in adulthood (Chu, Rimm, \& Wang et al., 1998; \& 
Berenson, Srinivasisn, \& Bao et al., 1998). Denney-Wilson, Hardy, \& Dobbins et al. (2008) found that obese adolescents were more likely to have two or more risk factors for T2DM and CVDS than their non-overweight counterparts. Adolescent males were more likely to have more than one risk factor than adolescent females especially if they were overweight or obese. In the present study, adolescent females reported significantly more risk factors than males, and overweight subjects had more risk factors than their underweight or normal weight counterparts. These findings on overweight are important since obesity during childhood and adolescence persists into adulthood. It is likely that the accompanying risk factors of hypertension, AN, hyperglycemia, and hyperlipidemia will be manifested in the adult years. Primary prevention programs are therefore necessary and may be implemented at the high school level.

Our results indicated that Jamaican adolescents may be at risk for T2DM and CVDs and that the BMI has a direct bearing on the risk factors of T2DM and CVDs. Therefore, measures used to reduce overweight and obesity during childhood and adolescence may help to reduce these risk factors. Simply monitoring subjects' weight through the early screening at the high school level and earlier, may help to reduce other related risk factors of T2DM and CVDs among this population. In view of our findings, we predict that our results of high BMI and presence of the METS will lead to the development of T2DM and CVDs among the young Jamaican population in later years. Preventive measures such as dietary intervention to reduce weight and consumption of saturated fats, increased PA and weight management among adolescents are needed during the school years. These interventions can target school children and adolescents in the school setting and at the national level. Jamaica spends about $6 \%$ of GDP on the 
treatment of diabetes and hypertension (PAHO Report, 2008). The economic cost of treating diabetes in the USA was estimated at \$174 billion in 2007 (ADA, 2007 http://www.diabetes.org). Americans diagnosed with diabetes have medical expenditures that are 2.3 times higher than in the absence of diabetes. What is of concern is that diabetes is highly related to poor nutrition and lifestyle risk factors some of which are modifiable through early screening and interventions.

\section{Waist Circumference and Waist-to-Hip Ratio}

The relationship between WC and WHR on the risk factors of T2DM and CVDs were examined since earlier studies showed that central adiposity (high WC and WHR) are associated with CVDs risk factors such as hyperinsulinemia, hypertriglyceridemia and hypertension (Gopalan, 1998, Ramchandaran, 2004). High WC was assessed using the NCEP Adult Treatment Panel III values of $\geq 94 \mathrm{~cm}$ and $\geq 80 \mathrm{~cm}$ for males and females respectively. These represented North American adult cut-off values for WC. Nevertheless, high WC was significantly related to high FBG, BP, BMI, presence of AN, positive family history of obesity, and the higher total number of risk factors in our study. Higher waist-to-hip ratios (WHR) were significantly associated with high BMI, and low PA. Unlike earlier studies (Gopalan, 1998, Ramchandaran, 2004), we did not measure insulin resistance (IR) or triglycerides. However, we found positive correlations between high WC and high FBG, BP, BMI, and family history of obesity. Our study provides preliminary data for further comparisons of Jamaican adolescents with US counterparts and in the validation of acceptable WC and WHR among Jamaican adolescents. 
Interventions aimed at reducing WC and WHR among Jamaican adolescents include the same approaches needed for reduced overweight and include diet planning and increased physical activity. It is likely that Jamaican adolescents do not see themselves as at risk with high WC and WHR since it is culturally accepted to have larger body frames, especially around the hip.

As expected, WC strongly correlated with BMI. However, research indicated that even some individuals with low BMI tend to have high abdominal fat and are prone to T2DM, CVDs and hypertension (IOTF, 2006). In a study of Asian Indians, Gopalan (1998), found that up to $20 \%$ of the subjects although not overweight, had central obesity that puts them at risk for CVDs, T2DM, and hypertension. We felt that measuring BMI alone was not sufficient for our study population since BMI is a composite of muscle and body fat and it is likely to find low BMI with high WC and WHR as found in other populations (Ramachandran et al., 1997).

There is conflicting research on WC and BMI as indicators of T2DM and CVDs (Ramachandran et al., 1997; Reaven, 2005). A recent study found that WC predicted T2DM but was not a good predictor of CVDs (Janiszewski, Jansen, and Ross, 2007). Although earlier research indicated that WC highly correlated with IR, the use of WC over BMI is still controversial. Denney-Wilson et al (2008) found that BMI was a better predictor of T2DM and CVDs than WC. Alberti et al. (2005) suggested that as long as BMI is over $30 \mathrm{~kg} / \mathrm{m},{ }^{2} \mathrm{WC}$ does not need to be measured. Despite the conflicting results on the use of WC compared to BMI in determining at risk populations for T2DM and CVDs in previous studies, we concluded that WC may be used to screen Jamaican adolescents since $\mathrm{WC}$ is an indicator of abdominal fat whereas a high BMI is a composite 
of body fat and muscle. Further, Asian studies indicated that individuals with low BMI had high WC. Waist Circumference appeared to be more strongly associated with other risk factors for T2DM and CVDs than BMI in our sample.

\section{BMI and Family History of Obesity}

Obesity is well known to have a strong relation to family history of obesity. The risks of being obese increases by $66 \%$ if both parents are obese, and falls to $50 \%$ if one parent is obese. If neither parent is obese, the risk of being obese is only $9 \%$ (Ponder, Sullivan, \& McBath, 2000). We hypothesized that subjects with family history of overweight and obesity would also be overweight and obese. Family history of obesity was measured using a silhouette of body figures. These figures represented various BMI levels for males and females. Adolescents selected the silhouettes that best represented their parents. $\mathrm{BMI}>25 \mathrm{~kg} / \mathrm{m}^{2}$ for mothers and fathers was considered at risk and BMI $<$ $25 \mathrm{~kg} / \mathrm{m}^{2}$ no risk to create a new variable called family history of obesity by combining BMI $>25 \mathrm{~kg} / \mathrm{m}^{2}$ of mother and father. Significant correlations were found between family history of obesity and subjects' BMI. Although a smaller percentage of adolescents than parents in our study were overweight, the adolescents were considerably more obese than their parents. In our study, eleven (3.9\%) adolescents were classified as morbidly obese based on actual BMI measures.

Findings from our study on Jamaican adolescent on patterns of overweight and obesity are similar to earlier findings from the Heart Foundation of Jamaica Supermarket Screening Program (SSP), with over $30 \%$ of adults reported as being overweight and over 
$20 \%$ being obese. The SSP found a small percentage (2\%) of morbidly obese adults.

There was no report of morbid obesity among the fathers, and only one mother was reported as morbidly obese in our study. Up to $4 \%$ of mothers were found to be underweight, compared to $24.6 \%$ underweight adolescents in our sample. This relationship between subjects' and parents' overweight/obesity has strong implications of healthcare approaches in the future. Further, the cultural dynamics are important as subjects may not conceive themselves as being at risk for chronic diseases and may not see the need for change. Any intervention therefore needs to address weight reduction in relation to development of chronic diseases.

Family history of obesity is important to the prevention of T2DM and CVDs since children are more likely to become obese if one or more parent is overweight or obese (The United States Surgeon General's Report, US Department of Health and Human Services, 2006) and overweight in children has been shown to transcend into adulthood which increases the risk of T2DM and CVDs (Whitaker et al., 1997; Deckelbaum \& Williams, 2001). These findings are significant to dietitians and other health professionals who can tailor programs to address overweight and obesity among the parents. Intervention measures are also needed for adolescents whose parents are overweight or obese since there is a strong relationship between family obesity and obesity in off-springs.

\section{Diet and Nutrient Intakes}

In this study, an in-depth analysis of the macronutrients and types of fats was presented because of their association with overweight and the risk factors of T2DM and 
CVDs. Food intake data were collected for a 24-hour period using the USDA Multiple Pass Method. This method has been validated in a study of adults ages 21-65 years (Conway, et al., 2003). We pilot tested the macronutrient intakes of 40 Jamaican adolescents and compared 24 hour intakes to 48 , and 24 to 72 hours, using The USDA Multiple Pass Method. Analyses revealed no significant differences between 24 and 48 hours, and 72 hour intakes for all macronutrients, fiber and total energy intakes. Thus the 24 hour recall was considered suitable for correctly estimating the usual energy and macronutrient intakes of our population. Intakes of macronutrients and fiber had significant negative correlations with a number of risk factors for T2DM and CVDs and intakes of select nutrients at the .05 level of significance. However, when we controlled for type 1 error we found significant associations only between fiber and AN, and WC and total fat intake.

Intakes of fiber had a significant negative correlation with AN in our study. This is not surprising since earlier epidemiological studies have shown that diets rich in whole grains lower the risk of CVDs and T2DM (Liu, 2002; liu et al., 2000; Salmeron et al., 1997) and the consumption of whole grain cereals has also been associated with reduced risk of CVDs among men and women (Loke, 2005; Liu, 2002; Gross et al., 2004). We expected higher consumption of fiber since the island abounds with tropical fruits and vegetables. However, mean intake of fiber was $14.4 \pm 9.6 \mathrm{~g} / \mathrm{d}$ which is below the Adequate Intake for adolescent males (38 g/d) and females (26 g/d) (Food and Nutrition Board, Institute of Medicine, National Academies, USDA Dietary Reference Intake, 2002). A limitation of our study may have been the timing of the data collection since it was done within weeks of Hurricane Dean which destroyed fruit trees and vegetables. In 
addition, data on fruit and vegetable intakes of Jamaicans are sparse. In a recent report by the Washington representative of the Pan American Health Organization (PAHO, 2008), reference was made to the low intakes of fruits and vegetables as a major risk factor for deaths and disabilities in the region. It would appear that even outside of a natural disaster, the usual intakes of fruits and vegetables of this population are low. Further research is needed on the sources of fiber and relation to absence of AN. Exercise and improved diet can help to lower insulin levels and can improve AN (Reaven, 1988).

Although no significant associations were found for the types of fat in our study, total fat was a composite of all types of fats. We examined the consumption of types of fats since earlier research indicated that the types of fats consumed rather than the amounts are associated with health risks and benefits. Other studies have found that saturated fats are associated with CVDs risks whereas intakes of omega-3 fatty acids seem to have a protective property ( $\mathrm{Hu}, 2000$; Howard, 2000; Mayer-Davis, 2000), and that saturated fats increased the severity of CVDs risks (Pan, Liu, \& Wang, et al, 1991; Wang, Tam, \& Mc Creddie et al, 1994; Hsia, Duncan, \& Schob et al, 2000). These studies looked at serum cholesterol, HDL-C, LDL-C and TG and the severity of CVDs using angiographs which were not possible measures for our study, thus a comparison could not be made. We found a significant but weak negative correlation between total fat and WC $(r=-.18, p=.003)$. Higher WC was associated with lower fat intakes in our study. It is likely that high WC is associated with fat stored from other macronutrients and not necessarily from high dietary intakes of fat per se. 
It was expected that Jamaican diets would be high in saturated fats similar to the diets of the resource-rich countries. However, overall fat intakes were within the recommended macronutrient distribution range for saturated fat. The intakes of saturated fats compared to other studies may be due to the higher cost of food items which contain saturated fats (milk, cheese, eggs, meats) for this sample. Examination of the diets for the types of foods consumed and the percentage of calories and from gram amounts of fats present is needed in future studies.

The lack of association between types of fats and other risk factors examined in the current study may be attributed to observation bias. It is likely that subjects reduced, or inadvertently under-reported fat intakes for the purpose of the study. Adolescents were aware that they would be questioned on their food intake on data collection day and they might have eaten low-fat foods. We considered our evaluation of a 24-hour recall of fat intakes suitable based on results of our pilot test. The pilot test revealed no significant differences between fat intakes over a 24 and 48 , or 24 and 72 hours. Although there is no evidence that consumption of dietary fat leads to T2DM, high fat intakes contribute to obesity which is a major risk factor of T2DM (Hu, 2000). Further, high fat intake has been shown to increase cholesterol levels which are associated with pre-mature heart disease by increasing CVD risks (Hu, 2000; Howard, 2000; Mayer-Davis, 2000).

No significant correlations were found between the risk factors for T2DM and CVDs and the intakes of omega- 3 and omega- 6 fatty acids in this study. This may be due to the extremely low intakes of these fatty acids. This was not surprising because it is a common practice for poor Jamaican families to consume meals without any animal products such as eating cabbage with a number of starchy roots instead of having a 
meat/fish dish. Data from NHANES III using a single 24 hour recall revealed that $25 \%$ of US population reported no EPA and DHA intakes (Wang, Chung, Lichtensin et al., 2004). Similar to the NHANES III study, $76 \%$ of our study subjects reported intakes of omega-3 fatty acids though the amounts were small. It must be noted that fish which contains omega-3 fatty acids are mainly from the "oily fish group" with darker colored flesh such as mackerel, herrings and trout. However, intakes of fish in this data set included more white fish (cod, snapper) which lack omega-3 fatty acids. Further, despite the fact that this is an island and we expected it to be abound with seafood, the cost of fish is extremely high and lies outside the reach of many poor Jamaican families.

Overall, intakes of all macronutrients fell within the Average Macronutrient Dietary Range (AMDR) for American and Canadian diets despite findings of high BMI in this sample. Usual intakes of macronutrients might have been reduced the day before the data collection. Further research is needed on the appropriateness of the AMDR ranges for fats. The highest intake of saturated fat was provided by the lunch meal which was consumed at school. Research is needed on the overall diet quality and the relationship with physical activity and the factors for T2DM and CVDs in Jamaican adolescents.

Comparison of Finger Prick vs Venous Blood

Earlier studies examined capillary whole blood versus venous blood as a quick method of making early diagnoses for patients in emergency care. Using finger prick for testing blood samples for FBG, TC, and $\mathrm{HbAlc}$ is easier to perform, less invasive, requires very little training, and provides quick feedback. In this study, the two methods 
were compared to determine if capillary blood testing of FBG, TC, and $\mathrm{HbAlc}$ could be used as a substitute for venous testing in detecting risks of T2DM and CVDs among adolescents on site and in the school setting. For finger prick testing to be used instead of venous blood samples, there should be good agreement between the two methods, and the results should meet specific standards of variability between finger prick and the reference standards. Further, validation of these tests requires high sensitivity and specificity. High sensitivity indicated that the tests correctly identified the proportion of persons who tested positive. TC had the highest sensitivity followed by FBG, and HbA1c. The high correlations of finger prick and venous TC were evidence of the ability of finger prick TC to identify subjects at risk.

In our study significant mean differences were found between finger prick and venous methods of testing for TC and HbAlc $(p=.001)$ but not for FBG $(p=.185)$ when tested by paired sample t-test. However, correlation was strong for $\mathrm{TC}(\mathrm{r}=.80, \mathrm{p}<.001)$. The Bland Altman plot indicated good agreement for all three tests with $95 \%$ or more of the scores falling within \pm 2 SDs. Good agreement between both methods indicated that finger prick testing may be used as a surrogate in the early screening for risk factors of T2DM and CVDs among Jamaican adolescents.

Comparison of capillary FBG and venous FPG in our study shows conflicting results with earlier studies. Funk, Chan, \& Lutz (2001) found poor correlation for FBG between capillary and venous blood samples of healthy volunteers. Other studies on critically ill patients' venous blood samples were found to be more accurate than capillary blood (Sylvan, Pokorny, \& English, 1995; \& Thomas, Gough, \& Benson, 1994). 
In our study, no significant difference was found between capillary and venous blood testing of FBG in healthy adolescents.

Sacks et al. (2002) found $20 \%$ variability between capillary blood glucose (measured by self-monitoring devices such as the Acu Chek Advantage used in this study) and venous FPG at levels of $>4.2 \mathrm{mmol} / \mathrm{L}$. The variability of $20 \%$ did not meet the ADA's standard of variability between both methods being $<5 \%$. However, whole blood levels of $100-126 \mathrm{mg} / \mathrm{dL}$ representing above normal levels of FBG showed a percentage bias of $-2.4 \%$ between whole blood and plasma samples in the current study. Therefore results for variability between the two methods of testing FBG in our study met the most recent ADA standard (ADA, 1994) recommendation of $<5 \%$.

The International Federation of Clinical Chemistry recommended that blood glucose levels referenced to plasma should be approximately $11 \%$ higher than those referenced to whole blood (D'Orazio et al., 2005). Further studies are needed to compare these methods to determine variability of results. Our study did not allow for repeatability of the blood tests and this one-time measurement may not be adequate to determine variability between the methods of blood testing.

Studies have shown lower precision with finger prick testing for TC as compared to standardized laboratory testing (Dipeolu, 2006). Other studies, (Kafonek, 1992; Costanza et al., 2005) found that biological variations of TC, TG, HDL-C, and LDL-C were the same for venous and capillary testing. The current study revealed that fingerprick blood TC was significantly different from venous blood but showed good agreement between the two methods of testing in the Bland Altman Plot. 
Our study did not meet the standard requirements for percentage bias between methods of blood testing for TC. Therefore finger prick testing of TC was considered to be suitable for early screening but was not recommended for making clinical decisions. Differences between methods of blood testing of TC might have related to dilution of interstitial fluid in the finger prick samples giving a lower cholesterol concentration since the subjects underwent three finger prick tests in quick succession on the same day. Despite these discrepancies, we believe our tests for TC is suitable as a preliminary screening among our study population.

A limitation of the study was the measurement of TC only. HDL-C, LDL-C or TG was not measured. Measurement of a complete lipid profile would have been ideal but the instrument for measuring finger prick TC could not be used to measure TG or HDL-C. Previous studies show that people with T2DM have abnormal lipid profiles with elevated LDL-C, TG and decreased HDL-C levels which put them at risk for CVDs (Assmann, Cullen \& Schulte, 2002). Another study showed that treatment to increase HDL-C levels was found to be more effective than lowering LDL-C levels in patients with T2DM (Windler, 2005) since HDL-C returns cholesterol to the liver for excretion via bile. The Joint British Societies Guidelines (JBS) suggested that in the absence of HDL-C measures, total cholesterol value should be used alone on the same scale and the value taken as one (Dipeolu, 2006). Comparison of a complete lipid profile was not possible for this sample since we could not measure a complete lipid profile by finger prick testing. Although earlier studies recommended a complete lipid profile for clinical diagnosis, we consider the measures of TC to be useful. Lowering TC and increasing HDL-C is the recommended treatment for patients with abnormal lipid profiles and risk 
of CVDs. Similar to our findings, Dipeolu, (2006) found that capillary testing is easy to perform and less costly than venous testing however, it has lower specificity and sensitivity.

Our study was unprecedented in its examination of finger prick testing for $\mathrm{HbAlc}$ among Jamaican adolescents. We found above normal levels of $\mathrm{HbA} 1 \mathrm{c}$ by finger prick blood testing compared to venous blood. Higher finger prick values of HbAlc may be due to instrument error. A previous study which compared four commercial point-ofcare $\mathrm{HbAlc}$ analyzers with laboratory testing found that the Nycocard system had the lowest precision (Hawkins, 2003). In our study, the percentage bias for HbAlc was $12.6 \%$ compared to $2-3 \%$ for established physiological limits and $3-4 \%$ for the National Glycohemoglobin Standardization Program (Sacks, 2005). Another possible explanation for the significant difference between finger prick and venous testing of $\mathrm{HbAlc}$ in our sample may be related to the outliers in the finger prick versus venous blood samples when the complete range of values was tested. When the scores were standardized, both finger prick and venous measures showed good agreement between methods of blood testing.

Unlike the higher finger prick $\mathrm{HbA} 1 \mathrm{c}$ values compared to venous values found in the present study, Schwartz et al. (2005) in a study of 147 diabetic patients found consistently lower $\mathrm{HbA} 1 \mathrm{c}$ results for capillary blood testing than venous blood methods. The study was conducted to determine if same day testing of $\mathrm{HbA} 1 \mathrm{c}$ using capillary blood from finger prick samples could be used to improve diabetes care management. Sicard \& Taylor (2005), compared finger prick measures of HbAlc with standardized laboratory procedures. The researchers found strong correlations between both methods. 
However, similar to Schwartz et al (2005) study, the sample included known diabetics. It is likely that more elevated $\mathrm{HbAlc}$ values would be present in known diabetics. The current study excluded previously diagnosed diabetics in the sample. The strength of our study therefore lies in its ability to detect high levels of $\mathrm{HbAlc}$ in seemingly healthy volunteers so that early remedial actions can be taken.

Despite the high levels of $\mathrm{HbAlc}$ found in our sample, we do not recommend testing of HbA1c solely in diagnosing diabetes among our population. A search of the literature showed there is still no consensus that tests for $\mathrm{HbAlc}$ alone are conclusive for a diagnosis of diabetes. The International Federation for Diabetes and the WHO have not agreed on the use of $\mathrm{HbAlc}$ only in the diagnosis of diabetes. However, $\mathrm{HbAlc}$, when used with other measures such as FPG and BMI can be effective for screening in this study population which is similar to the findings of Norberg et al., (2006).

Finger prick testing was economical, costing one third that of venous blood laboratory testing for the same measures in this study. Finger prick testing produced results within a few minutes and can be carried out on site in a convenient location such as in the "sick bay" in the school setting. Therefore it is an economical, fast and effective choice for a developing country. Most secondary schools in Jamaica employ a school nurse who can be trained to conduct these tests on site. As supported by the literature, obesity among adolescents is a strong indicator of the development of T2DM and CVDs in later years. Students who are overweight /obese can be further monitored by these finger prick tests in the school setting with the permission of the respective government agencies, and then referred for clinical follow-up as needed. Our results showed that screening of adolescents for high $\mathrm{FBG}, \mathrm{TC}$ and $\mathrm{HbAlc}$ is feasible within a school setting. 
Results of finger prick FBG and TC were more accurately and economically performed when compared to the higher cost of testing for HbAlc among this sample. Further, finger prick testing of $\mathrm{HbAlc}$ overestimated the subjects at risk for high $\mathrm{HbA} 1 \mathrm{c}$ in our study.

\section{Conclusion}

Jamaican adolescents are at risk for T2DM and CVDs similar to other ethnic minority groups in the US. They also tend to have a cluster of risk factors for T2DM and CVDs as seen in other studies. In this study, over $60 \%$ of the sample had three or more risk factors for T2DM and CVDs despite their weight status. Over 90 percent of the overweight/obese adolescents reported three or more risk factors of T2DM and CVDs. BMI and WC strongly predicted some of the risk factors for T2DM and CVDs. One third of our sample had BMI $\geq 25$ which is twice the percentage of overweight adolescents in the USA. The presence of AN was found in over $40 \%$ of the sample and correlated with high BMI as seen in other study populations of adolescents. Macronutrient intakes did not relate to the risk factors of T2DM and CVDs as expected, despite findings of high levels of BMI. Ethnicity, gender, place of residence and income levels were weak predictors of the risk factors of T2DM and CVDs.

Of the three biomarkers tested by finger prick and venous blood, FBG appeared to be most suitable for the early screening of adolescents in the school setting. There was no significant mean difference between the finger prick and venous measures of FBG and the Bland Altman Plot showed good agreement between the means of both methods of blood testing. Despite the significant mean differences found for finger prick and venous 
testing of TC and $\mathrm{HbAlc}$, the Bland Altman Plot showed good agreement for these measures which suggested that they may be used with caution in the early screening of adolescents for risk factors of T2DM and CVDs in a school setting.

Finger-prick screening is a suitable method for the developing countries with limited resources. This study showed that early screening can be carried out within the school setting with permission from the respective agencies and the parents. This type of screening can be routinely carried out by the respective Government Ministries. Finger prick screening, in combination with other measures such as BMI, AN, perception of weight status, and WC is a suitable method for the resource poor countries since it is more economical, less time consuming, yields rapid results, and are less feared by adolescents compared to venous blood draws.

Finally, with the increase in cases of diabetes in Jamaican adults, and the rising cost of treating diabetes and its complications, early detection of risk factors during the school years is recommended. Our study showed that anthropometric measures and family history of disease identified more subjects at risk for T2DM and CVDs than did the biomarkers. Therefore intervention studies can be geared at minimizing the risk factors of T2DM and CVDs instead of just treating biochemical indices.

\section{Strengths and Limitations}

The study was the first of its kind that examined Jamaican adolescents for a multiplicity of risk factors for T2DM and CVDs. Thirteen risk factors were examined in this study. Prior to this study, no emphasis was placed on evaluating risk factors for T2DM and CVDs in children and adolescents by the agency responsible for diabetes 
research in Jamaica; The Diabetes Association of Jamaica. Similarly, the Heart

Foundation of Jamaica did not conduct studies involving adolescents. By involving these agencies in the data collection process, they were sensitized to the development and early prevention of these conditions in adolescents and have set a precedent for further research involving this population.

Testing for the presence of AN was a novel part of the study and was new to the agencies and study population involved. Although participants and agency leaders were aware of the physical signs of AN, no one associated this with the risk of T2DM. The study was the first of its kind to analyze the relationship of the risk factors for T2DM and CVDs with nutrient intakes in this population. A comprehensive analysis of nutrient intakes of this population in terms of macronutrient contributions by meal types was presented. Finger prick and venous blood testing for FBG, TC, and HbAlc indicated good agreement between methods of testing which proves that early screening can be carried out within the school system at a minimal cost to parents.

The study which aimed at identifying Jamaican adolescents at risk for T2DM and CVDs may be limited in some areas. First, some of the more common measures for detection of the metabolic syndrome such as insulin resistance (IR) and triglycerides (TG) were not measured due to high cost. It was not feasible to replicate the more expensive laboratory tests. Low cost, accurate measures have been shown to be suitable for screening but not for making diagnoses. TG was measured on the sub-study sample and found to be below $200 \mathrm{mg} / \mathrm{dL}$ for all 59 subjects of the sub-sample.

Second, family history of obesity was determined by having students select a silhouette of various BMI levels representing their perceptions of their mothers and 
fathers BMI. Validation of this silhouette of the parents by comparing them with actual BMI measures was not done. Third, the scale used to measure PA measured frequency of activities engaged in, but did not measure amount of time spent in physical activity or the amount of energy expended on these activities. For future research, we recommend modification of this tool to make it more accurate and analyzable.

Fourth, our method of data collection for food intake, and timing of the data collection limited our findings. The 24 -hour recall might not have adequately represented the true food intakes. Subjects were aware they would be questioned on their food intakes as this might have introduced observer bias. They might have modified their previous day's food intake. Data were collected within weeks of hurricane Dean which destroyed much of the fruits and vegetables and may not have represented the true fiber intakes of this population.

The study did not allow for repeatability in comparing the two methods of blood testing. Agreement between two methods which determines if one can be substituted for another requires good repeatability between both methods. If one method has poor repeatability, then agreement is affected. Although blood samples were collected on the same day, venous samples were taken by one laboratory and finger prick samples by another. However, all venous samples were taken and tested by the same laboratory. Statistical errors were controlled for by selecting a .05 probability level in computing results. 


\section{Recommendations}

Prevention of chronic diseases such as T2DM and CVDs can greatly reduce healthcare costs for governments in many countries especially in the resource- poor countries, and increase quality of life. Recent reports showed that cases of diabetes have doubled in Jamaica between 1980-2000. Jamaica spends approximately 42 billion dollars annually on the treatment of diabetes and hypertension (PAHO, 2008).

Formerly, a preventive approach for T2DM was to screen individuals over 45 years old (Expert Committee on the Diagnosis and Classification of Diabetes mellitus, 1997). However, more recent research suggests screening children over the age of 10 years for T2DM, if the child is overweight and has two other risk factors (family history, race/ethnicity, and signs of insulin resistance). It is also recommended that screening for T2DM should be carried out every two years (Sacks, et al., 2005). CVDs are not manifested in the early years but there is evidence that the atherosclerotic lesions associated with CVDs in later years begin in early childhood (Gungor et al., 2005; Steinberger et al., 2003) therefore screening of adolescents for risk factors of CVDs is also recommended. Other recommendations from our study include:

- Screening school children for risk factors of T2DM and CVDs bi-annually within the school system. This is manageable since all parties concerned gave their full support to the study. The Ministry of Health and Environmental Control and the Ministry of Education, Youth and Culture did not oppose this screening within the schools. Parents did not object to having their child tested at schools and children were quite willing to undergo testing. Principals and school nurses were supportive and did not object to this type of screening. 
- Sensitizing the school nurses to the growing problems of childhood obesity and the related metabolic syndrome. The school nurses can be trained to conduct these finger prick tests with the permission of the Ministry of Health and Environmental Control and the Ministry of Education, Youth, and Culture. Cost of testing can be made part of the cost-sharing efforts in the schools between parents and government subsidies.

- Conducting follow-up testing and monitoring for children and adolescents who are overweight and obese, those who present with grade 3 and 4 for severity of AN on the neck, high WC, FBG, TC, HbAlc levels, pre-hypertension and hypertension. This can be achieved by working with the school nurses and the guidance counselors who will bring this to the attention of the parents.

Identification of these risk factors can help in the planning of early intervention to improve the long-term outcomes of these chronic diseases. Simple monitoring of body weight is achievable at no extra cost to the schools.

- Training the school nurses to recognize the physical signs for $\mathrm{AN}$ which requires no additional cost to the school or parents.

- Re-introducing physical education to all grades in high schools to reduce the growing rates of overweight and obesity.

- Teaching students about the importance of diet for the prevention of T2DM and CVDs. This can be added to the nutrition curriculum at the high school level. Nutrition needs to be offered as a core so all can benefit from this subject. It is customary for students to select specific subjects at the end of grade nine, and many might not choose food and nutrition for further studies. Therefore, nutrition 
knowledge on these chronic, nutrition-related diseases is best introduced at grade nine.

\section{Suggestions for Future Studies}

Future studies may require validation of the silhouettes used for determining parents' BMI on a group of parents. Measures of PA need to be more comprehensive since the scale used did not take into account amount of time spent on an activity, amount of energy expended, and with a low cut-off point of two activities per week seem inadequate. The scale classifies students as physically active despite having high BMI.

Repeatability of the methods of blood testing may be carried out and have one laboratory conduct all tests to reduce variation among tests results. Coordination of three different agencies on data collection days was challenging therefore for future studies one agency/laboratory should be used for all blood tests. More accurate testing for determining the presence of the metabolic syndrome, using other indices such as raised LDL-C and TG as recommended by the International Diabetes Federation are to be included instead of TC as was done in this study. Studies indicate that IR is a good indicator of the metabolic syndrome in adolescents and is a strong marker for T2DM. However, IR was not measured in this study.

Our study has set the framework for further studies on nutrition-related chronic diseases among Jamaican adolescents. Based on evidence from this research on the prevalence of risk factors for T2DM and CVDs among Jamaican adolescents, there is need to be develop interventions to prevent risks leading to these costly and fatal chronic diseases. 


\section{LIST OF REFERENCES}

Acanthosis Nigricans: The Education and Screening Program (ANTES). http://www.panam.edu/de[t/tmbhco Retrieved 7/30/2008.

Alberti, K.G.M.M., \& Zimmet, P.Z. (1998). For the WHO Consultation (1998). Definition, diagnosis, and classification of diabetes mellitus and its complications. Part I: diagnosis and classification of diabetes mellitus, Provisional report of a WHO Consultation. Diabetic Medicine, 15, 539-553.

Alberti KGMM, Zimmet P, \& Shaw J. (2005). The metabolic syndrome- a new worldwide definition. The Lancet, 366, 1059-1062.

Allison, D. E., Fountaine, K. R., Manson, J.E., Stevens, J., \& VanItalie, T.B. (1999). Annual deaths attributable to obesity in the United States Of America. Journal of the American Medical Association, 282(16), 1530-1538.

Altman, D.G., \& Bland, J.M. (1983). Measurement in medicine: The analysis of method comparison studies. Statistician, 32, 301-317.

American Diabetes Association (2000). Type 2 diabetes in children and adolescents (Consensus in Statement). Diabetes Care, 23, 381-389.

American Diabetes Association (2005). Clinical Practice Recommendations 2005. Diabetes Care, 28, S1-79.

American Obesity Association. Childhood Obesity. http://www.obesity.org/subs/childhood/prevalence.shtml Retrieved 6/21/2006.

Assmann, G., Cullen, P., \& Schulte, H.(2002). Simple scoring scheme for calculating the risk of acute coronary events based on the 10-year follow-up of the prospective cardiovascular munster (PROCAM) study. Circulation, 105, 310-315.

Bao, W., Srinivasan, S.R., \& Berenson, G.S. (1996). Persistent elevation of insulin levels is associated with increased cardiovascular risk in children and young adults: The Bogalusa Heart Study. Circulation, 93, 54-59.

Bavdekar, A., Yajnik, C.S., Fall, C.H., Bapat, S., Pandit, A.N., Deshpande, V, et al. (1999). Insulin resistance syndrome in 8-year old Indian children: Small at birth, big at 8 years or both? Diabetes Care, 48, 2422-2429.

Bensike, JF., Levy, RI., \& Kelsey, K.F. (1984). Effects of therapy with cholestyramine on progression of coronary arteriosclerosis: results of the NHLBI Coronary Intervention Study. Circulation, 69, 313-324. 
Berenson, G.S., Wattigney, W.A., Bao, W., Srinivasan, S.R., \& Radhakrishnamurthy, B. (1995). Rationale to study the natural history of heart disease: the Bogalusa heart Study. American Journal of Medical Science, 1, S22-S28.

Berenson, G.S., Srinivasisn, S.R., Bao, W., Newman, W.P. III, Tracy, R.E., \& Wattigney, WA. (1998). Association between multiple cardiovascular risk factors and atherosclerosis in children and young adults. The Bogalusa Heart Study. New England Journal of Medicine. 338(23), 1650-1656.

Berry, D., Sheehan, R., Heschel, R., Knafl, K., Melkus, G., \& Grey, M. (2004). Family based interventions for childhood obesity: A review, Journal of Family Nursing, 10, 429-449.

Berry, D., Urban, A., \& Grey, M. (2006). Understanding the development and prevention of type 2 diabetes in youth (Part I). Journal of Pediatric Health Care, 20(1), 3-10.

Blades, B., \& Garg, A. (1995). Mechanisms of increase in plasma triacylglycerol concentrations as a result of high carbohydrate intakes in patients with noninsulin-dependent diabetes mellitus. American Journal of Clinical Nutrition, 62, 996-1002.

Blakely, T., Hales, S., Wilson, N., \& Woodward, A. (2004). Distribution of risk factors by poverty. In Ezzati, M., Lopez, A.D., Rodgers, A., Murray, C.J.L., editors. Comparative quantification of health risks: Global and regional burden of disease attributable to selected major risk factors. Geneva: World Health Organization. pp 1941-2127.

Bland, J.M., \& Altman, D.G. (1986). Statistical methods for assessing agreement between two methods of clinical measurement. Lancet, 8476, 307-310.

Bloomgarden, Z.T. (2004). Type 2 diabetes in the young: The evolving epidemic. Diabetes Care, 27(4), 998-1010.

Borders, T.F., Rohrer, J.E., \& Cardarelli, K.M., (2006). Gender specific disparities in obesity. Journal of Community Health, 31, 57-68.

Bowden, R.G., Kingery, P.M., \& Rust, D.M. (2004). Assessing risk using different cholesterol screening methods. Public Health 118(3), 225-229.

Broussard, B.A., Sugarman, J.R., Bachman-Carter, K., Booth, K, Stephenson, L., \& Strauss, K. (1995). Toward comprehensive overweight programs in Native American communities. Obesity Research, 3, 289s- 297s. 
Bulik, C.M., Wade, T.D., Heath, A.C., Martin, NG., Stunkard, A.J., \& Eaves, L.J. (2001). Relating body mass index to figural stimuli: population-based normative data for Caucasians. International Journal of Obesity and Related Metabolic Disorders, 25(10), 1517-1524.

Burke, J.P., Hale, D.E., Hazuda, H.P., \& Stern, M.P. (1999). A quantitative scale of Acanthosis Nigricans. Diabetes Care, 22(10), 1655- 1659.

Calderon, K.S., Yucha, C.B., \& Schaffer, S.D. (2005). Obesity-related cardiovascular risk factors: Intervention recommendations to decrease adolescent obesity. Journal of Pediatric Nursing, 20(1), 3-14.

Cappon, J.P., Ipp, E., Brasel, J.A., \& Cooper, D.M. (1993). Acute effects of high fat and high glucose meals on the growth response to exercise. Journal of Clinical Endocrinology and Metabolism, 76, 1418-1422.

Center for Disease Control and Prevention, Department of Health and Human Services. Body Mass Index: About BMI for children and teens. http://www.cdc.gov/nccdphp/dnpa/bmi/children_BMI/about_children_BMI.htm Retrieved 6/21/2006.

Center on Budget and Policy Priorities. (2004). Number of Americans without insurance reaches highest levels on record. center@cbpp.org. Retrieved 6/28/ 2006.

Certain, L.K., \& Khan, R.S. (2002). Prevalence, correlates, and trajectory of television viewing among infants and toddlers. Pediatrics, 109(94), 634-642.

Chan, A.Y.W., Swaminathan, R., \& Cockran, C.S. (1989). Effectiveness of sodium fluoride as a preservative of glucose in blood. Clinical Chemistry 35, 315-317.

Chen, W., Bao, W., \& Begum, S., Elkasabany, A., Srinivasan, S.R., \& Berenson, G.S. (2000). Age-elated patterns of the clustering of cardiovascular risk variables of syndrome $\mathrm{x}$ from childhood to young adulthood in a population made of black and white subjects: the Bogalusa Heart Study. Diabetes, 49, 1042-1048.

Choudbury, P. (2005). Adolescent onset of adult metabolic diseases. Journal of Indian Medical Association, 103(11), 630-637.

Christakis, D.A., Ebel, B.E., Rivara, F.P., \& Zimmerman, F.J. (2004). Television, video, and computer game usage in children under 11 years of age. Journal of Pediatrics, 145(5), 652-656. 
Chu, N.F., Rimm, E.B., Wang, D.J., Liou, H.S., \& Shieh, S.M. (1998). Clustering of cardiovascular disease risk factors among obese school children: The Taipel Children Heart Study. American Journal of Clinical Nutrition, 67(6),1141-1146.

Cohen, J. (1988). Statistical power analysis for the behavioral sciences, $2^{\text {nd }}$ edition, Hillsdale, New Jersey; Lawrence Earlbaum Associates. pp.4.

Cohen, M., Boyle, E., Delaney, C., \& Shaw, J. (2005). A comparison of blood glucose meters in Australia. Diabetes Research and Clinical Practice, 71, 113-118.

Cole, T.J., Bellizzi, M.C., Flegal, K.M., \& Dietz, W.H., (2000). Establishing a standard definition for child overweight and overweight worldwide: International survey. British Medical Journal, 320, 1240-1243.

Collins, R., Peto, R., \& MacMahon, S. (1990). Blood pressure, stroke, and coronary heart disease. Part 2, Short- term reductions in blood pressure: overview of randomized drug trials in their epidemiological context. Lancet, 335, 827-828.

Conway, J.M., Ingwersen, L.A., Vinyard, V.T., \& Moshfegh, A.J. (2003).

Effectiveness of the US Department of Agriculture 5-step multiple-pass method in assessing food intake in obese and non-obese women. American Journal of Clinical Nutrition, 77, 1171-1178.

Conway, J.M, Ingwersen, L.A., \& Moshfegh, A.J. (2004). Accuracy of dietary recall using the USDA Five-Step Multiple-Pass method in men: An observational validation study. Journal of the American Dietetic Association, 104(4), 595-603.

Cooper, R., Cutler, J., Desvigne-Nickens, P., Fortmann, S., Friedman, L. et al (2000). Trends and disparities in coronary heart disease, stroke, and other cardiovascular diseases in the United States: Findings of the National Conference on Cardiovascular Disease Prevention. Circulation 102, 3137-3147.

Costanza, M.C., Wolf, H., James, R.W., \& Morabia, A. (2005). In a quasisimultaneous assessment, imprecise monitoring and screening tests were improved. Journal of Clinical Epidemiology 58, 841-848.

Crocker, P.R.E., Bailey, DA., Faulkner, RA., Kowalski, K.C., \& McGrath R. (1997). Measuring general levels of physical activity: Preliminary evidence for the Physical Activity Questionnaire for Older Children. Official Journal of the American College of Sports Medicine, 29(10), 1344-1349.

Crespo, C.J., Smith, E., Troiano, R.P., Bartlett, S.J., Marcera, CA., \& Anderson, RE. (2001). Television watching, energy intake, and obesity in US children: results 
from the third National Health and Nutrition examination Survey, 1988-1994. American Pediatric Adolescence Medicine, 155(3), 360-365.

Cruz, P.D., \& Hud, J.A. (1992). Excess insulin binding to insulin-like growth factor receptors: proposed mechanism for Acanthosis Nigricans. Journal of Investigative Dermatology, 98(6), 82S-85S.

Dabelea, D., Hanson, R.L., Bennett, P.H., Roumain, J., \& Knowler, W.C. (1998). increasing prevalence of type II diabetes in American Indian children. Diabetologia, 41, 904-910.

Dalton, M., Cameron, AJ., Zimmet, PZ., Shaw, JE., Jolley, D., Dunstan, DW., \& Welborn, TA. (2003). Waist circumference, waist-hip ratio and body mass index and their correlation with cardiovascular disease risk factors in Australian adults. Journal of Internal Medicine, 254(6), 555-563.

Daniels, S.R. (2006). The consequences of childhood overweight and obesity. Future Child, Spring; 16(1), 47-67.

Davidson, M.B. (1995). Clinical implications of insulin resistance syndromes. American Journal of Medicine, 99(4), 420-426.

Deckelbaum, R.J., \& Williams, C.L. (2001). Childhood obesity: The Health Issue. Obesity Outreach, 9(4), 239S- 243S.

Delpeuch, F., \& Maire, B. (1997). Obesity and developing countries of the south. Medical Tropical (Mars), 57(4), 380-388.

Dennison, B.A., Erb, T.A., \& Jenkins, P.L.(2002). Television viewing and television in bedroom associated with overweight risk among low-income preschool children. Pediatrics, 109(6), 1028-1035.

Denney-Wilson, E., Hardy, L.L., Dobbins, T., Okeily, A.D., \& Baur, L.A. ( 2008). Body mass index, waist circumference, and chronic disease risk factors in Australian adolescents. Archives of Pediatric Adolescent Medical,162(6), 566-573.

Dietz, W.H., \& Bellizzi, M.C. (1999). Introduction: the use of body mass index to assess obesity in children. American Journal of Clinical Nutrition, 70, 123s-125s.

Dietz, W.H. (1997). Periods of risk in childhood for the development of adult overweight- What do we need to learn? Journal of Nutrition, 127, 1884S-1886S.

Dipeolu, P. (2006). The usefulness of random cholesterol capillary blood tests. Wadsworth Teaching Primary Care Trust. Review article. 
http://www.wadsworthpct.nhs.uk/pdf/public\%20health/CE/random\%20capillary \%20cholesterol\%20_March\%FINAL\%20VERSION.pdf Retrieved 01/24/2007.

Dowda, M., Ainsworth, B.E., Addy, C.L., Saunders, R., \& Riner, W. (2001).

Environmental influences, physical activity, and weight status in 8-16 year olds. Archive of Pediatric Adolescence Medicine, 155(6), 711-717.

D’Orazio, P., Burnett, R.W., Fogh-Andersen, N., Jacobs, E., Wolf, R.K. et al. (2005). Approved International Federation of Clinical Chemistry (IFCC) Recommendations on reporting results in blood glucose (Abbreviated), Clinical Chemistry, 51(9), 1573-1576.

Drewnowski, A., \& Damon, N. (2005). The economics of obesity: dietary energy density and energy cost. American Journal of Clinical Nutrition, 82(1), 265S-273S.

Drewnowski, A., \& Damon, N. (2005). Food choices and diet costs: an economic analysis. The Journal of Nutrition, 135(4), 900-904.

Drewnowski, A. (2007). The real contribution of added sugars and fats to obesity. Epidemiology Review, 29,160-171.

EDITORIAL - Health Ministry's warning on teen obesity. (2007). The Jamaican Daily Gleaner. Thursday June 28, 2007.

Enns, CW., Mickle, SJ., \& Goldman, J.D. (2002). Trends in food and nutrient intakes by children in the United States. Family Economics and Nutrition Review, 14(2), 56-68.

Epstein, F.H., \& Higgins, M. (1992). Epidemiology of Obesity. In: Bjorntorp P, Brodoff BN, eds. Obesity. Philadelphia: JB Lippincott Co, pp. 330-342.

Executive Summary of the Third Report of the National Cholesterol Education Program (NCEP) Expert Panel on detection, evaluation, and treatment of high blood cholesterol in adults (Adult Treatment Panel III). Journal of the American Medical Association, 285, 2486-2497.

Expert Committee on the Diagnosis and Classification of Diabetes Mellitus. Report of the Expert Committee on the Diagnosis and Classification of Diabetes Mellitus. (1997). Diabetes Care, 20, 1183-1201.

Fagot-Campagna, A. (2000). Emergence of type 2 diabetes mellitus in children: Epidemiological evidence. Journal of Pediatric Endocrinology \& Metabolism, 285, 2486-2497. 
Fagot-Campagna, A., Pettitt, D.J., \& Engelgau, M.M. (2000). Type 2 diabetes among North American children and adults: an epidemiological review and a public health perspective. Journal of Pediatrics, 136, 664-672.

Faul, F., \& Erdfelder, E. (1992). GPOWER: A priori, post hoc, and computer analyses for MS_DOS (Computer program). Bonn FRG: Bonn University, Dept. of Psychology. Food and Agricultural Organization of the United Nations. The nutrition transition and obesity.

http://www.fao.org?FOCUS?E?obesity?obes2.htm Retrieved 6/6/2006.

Food and Agricultural Organization. (1990). Conducting small-scale nutrition surveys: A field manual, Nutrition in Agriculture No. 24 Rome.

Food Composition Tables For Use in the English-Speaking Caribbean: Compiled by the Caribbean Food and Nutrition Institute, 1995.

Ford, E.S., Williamson, D.F., \& Liu, S. (1997). Weight change and diabetes incidences: Findings from a national cohort of US adults. Journal of Epidemiology, 146(3), 214-222.

Forshee, R. (2004). Methods of Surveying Diet and Physical Activity. The Economics of Obesity/ E-FAN-04-004 Economic Research Service/USDA pp 29-31.

Fountaine, K.R., Redden, D.T., Wang, C., Westfall, A.O., \& Allison, D.B. (2003). Years life lost due to obesity. Journal of the American Medical Association, 289 (2), 187-193.

Freedman, D.S., Dietz, WH., Srinivasan, S.R., \& Berenson, G.S. (1999). The relation of overweight to cardiovascular risk factors among children and adolescents: The Bogalusa Heart Study. Pediatrics, 103, 1175-1182.

Frick, M.H., Elso, O., Haspa, K. et al. (1987). Helsinki Heart Study: primary prevention trial with gemfibrozil in middle-aged men with dyslipidemia. New England Journal of Medicine, 317, 1237-1245.

Funk, D.I., Chan, L., \& Lutz, N. et al. (2001). Comparison of capillary and venous glucose measurements in healthy volunteers. Pre Hospital Emergency Care, 5, 275-277.

Galanis, D.J., Harris, T., Sharp, D.S., \& Petrovitch, H. (1998). Relative height, weight, change and risk of coronary heart disease in the Honolulu heart program. American Journal of Epidemiology, 147(4), 379-386. 
Gaskin, P.S., \& Walker, S.P. (2003). Obesity in a cohort of Black Jamaican children as estimated by BMI and other indices of adiposity. European Journal of Clinical Nutrition, 57 (3), 420-426.

Gillis, L.J., Kennedy, L.C., Gillis, A. M., \& Bar-Or, O. (2001). Relationship between juvenile obesity, dietary energy and fat intake and physical activity of. International Journal of Obesity, 26, 458-463.

Gopalan, C. (1998). Obesity in the Indian Urban"Middle Class." Bulletin of the Nutrition Foundation of India, 19(1), 1-5.

Gortmaker, S.L., Must, A., Sobol, A.M., Peterson., K., Colditz, GA., \& Dietz, WH.(1996). Television viewing as a cause of increasing obesity among children in the United States, 1986-1990. Archive of Pediatric Adolescence Medicine, $150(4), 356-362$.

Greenland, P., Bowley, N.L., Melklejohn, K.L., Doane, L., \& Sparks, CE. (1990). Blood cholesterol concentration: Fingerstick plasma vs. venous serum sampling. Clinical Chemistry, 36 (4), 628-630.

Gross, L.E., Li, L., Ford, E.S., \& Liu S. (2004). Increased consumption of refined carbohydrates and the epidemic of type 2 diabetes in the United States: an ecologic assessment. American Journal of Clinical Nutrition, 79, 774-779.

Guillaume, M. (1999). Defining obesity in childhood: current practice. American Journal of Clinical Nutrition, 70, 126S-130S.

Gungor, N., \& Arslanian, S. (2002). Pathophysiology of type 2 diabetes mellitus in children and adolescents: treatment implications. Treatment Endocrinology, $1(6), 359-371$.

Gungor, N., Saad, R., Janosky, J., \& Arslanian, S. (2004). Validation of surrogate estimates of insulin sensitivity and insulin secretion in children and adolescents. Journal of Pediatric, 144, 47-55.

Gungor, N., Thompson, T., Sutton-Tyrell, K., Janosky, J., \& Arslanian, S. (2005). Early signs of cardiovascular disease in youth with obesity and type 2 diabetes. Diabetes Care, 28 (5), 1219-1221.

Hanson, R.L., Nelson, R.G., McCance, D.R., Beart, J.A., Charles, M.A., Pettitt, D.J. et al. (1993). Comparison of screening tests for non-insulin-dependent diabetes mellitus. Archives of Internal Medicine, 153(18), 2133-2140. 
Hardy, L.R., Harrell, J.S.,.\& Bell, R.A. (2004). Overweight in children: Definitions, measurements, confounding factors, and health consequences. Journal of Pediatric Nursing , 19 (6), 376- 384.

Harris, M.I., Hadden, W.C., Knowler, W.C., \& Bennett, P.H. (1987). Prevalence of diabetes and impaired glucose tolerance and plasma glucose levels in the US population ages 20-74 years. Diabetes, 36, 523-534.

Hawkins, R.C. (2003). Comparison of four point-of-care HbAlc analytical systems against central laboratory analysis. Singapore Medical Journal, 44(10), 8-11.

Health Survey for England- The Health of Minority Ethnic groups. (1999). http://www.archive.official-documents.co.uk/document/doh/survey99/hse9903.htm Retrieved 7/3/2007.

Hennis, A., \& Fraser, HS. (2004). Diabetes in the English-Speaking Caribbean (Special report). Pan American Journal of Public Health, 15(2), 90-93.

Hirschler, V., Aranda, C., Oneto, A., Gonzalez, C., \& Jadinsky, M. (2002). Is acanthosis nigricans a marker of insulin resistance in obese children? Letter to the editor. Diabetes Care, 25, 2353.

Hoffmans, M.D., Krombout, D., deLezenne-Coulander, C. (1988). The impact of body mass index of 78,613 18 year-old Dutch men on 32-year mortality from all causes. Journal of Clinical Epidemiology, 41, 749-756.

Holms, S. (1979). A simple sequentially rejective multiple test procedure. Scandinavian Journal of Statistics, 6, 65-70.

Horgan, G. (2005). Exercise for children. Journal of Family Health Care, 15, 15-17.

Howard, BV. (2000). Evidence for dietary fat as a risk factor for type 2 diabetes. Presented at the $60^{\text {th }}$ Scientific Sessions of the American Diabetes Association; June 10, 2000, San Antonio, Texas.

Howarth, NC., Saltzman, E., \& Roberts, S.B. (2001). Dietary fiber and weight regulation. Nutrition Review, 59, 247-258.

Howarth, N.C., Terry, T., Huang, K., Roberts, S.B., Megan, A., \&.McCrory, A. (2005).

Dietary fiber and fat are associated with excess weight in young and middle-aged US adults. Journal of the American Dietetic Association, 105, 1365-1372.

Howdle, S., \& Wilkin, T. (2001). Type 2 diabetes in children. Nursing Standard, 15, $38-42$. 
Hsia, S.L., Duncan, R., \& Schob, A.H. (2000). Serum levels of high-density lipoprotein phospholipids correlate inversely with severity of angiographically defined coronary artery disease. Atherosclerosis, 152, 469-473.

Hu, F.B. (2000). Diet and type 2 diabetes: types of fats and types of carbohydrates. Presented at the $60^{\text {th }}$ Scientific Sessions of the American Diabetes Association; June 10, 2000, San Antonio, Texas.

Hulley, S.B., Cummings S.R., Browner, W., Grady, D., Hearst, N., \& Newman, T. (2001). Designing Clinical Research. Second edition. Lippincott Williams and Wilkens.

Ichinohe, M., Mita, R., Saito, K., Shinkawa, H., Nakaji, S., Coombs, M., Carney, A., Wright, B., \& Lewis-Fuller, E. (2005). The prevalence of obesity and its relationship with lifestyle factors in Jamaica. Tohoku Journal of Exp. Medicine, 207, 21-32.

Jamaica Facts and Figures (2005). http://encarta.msn.com/fact_631504789/Jamaica_Facts_and Figures.html. Retrieved 06/28/2006.

James, P.T., Leach, R., Kalamara, E., \& Shayeghi, M. (2001). Obesity Research, 9 (4), 228S-233S.

Janiszewski, P.M., Jansen, I., \& Ross, R. (2007). Does waist circumference predict diabetes and cardiovascular disease beyond commonly evaluated cardiometabolic risk factors? Diabetes Care, 30, 3015-3109

Kafonek, S.D., Donnovan, K., lovejoy, K.L. \& Bachorik, P. (1992). Biologic variation of lipids and lipoproteins in finger-prick blood. Clinical Chemistry 42 (12) 2002-2007.

Katz, A.S., \& Feldman, S.R. (2006). Acanthosis Nigricans in obese patients: Presentations and implications for prevention of atherosclerotic vascular disease. Dermatology Online Journal http://dermatology.cdlib.org/DOJvol16numl/original/acanthosis/katz.html. Retreived May 18, 2006.

Khan, A.I., Vasquez, Y., Gray, J., \& Frank, H.W Jnr. (2006). The variability of results between point-of-care testing glucose meters and the central laboratory analyzer. Archives of Pathology and Laboratory Medicine, 130,1527-1532. 
Kelly, A.S., Wetzeteon, R.J., Kaiser, D.R., Steinberger, J., Bank A.J., \& Dengel, D.R. (2004). Inflammation, insulin, endothelial function in overweight children and adolescents: the role of exercise. Journal of Pediatrics, 145, 731-736.

Kibbe, D.L., \& Rich, M. (2003). Childhood obesity: Advancing effective prevention and treatment. Washington, DC: National Institute of Health Care Management.

Kimm, S.Y., Barton, B.A., Obarzanek, E., McMahon, R.P., Sabry, Z., \& Waclawiw, MA. (2001). Racial divergence in adiposity during adolescence: The NHLBI Growth and Health Study. Pediatrics, 107, E34.

Klein, S., Burke, L.E., Bray, G.A., Blair, S., \& Allison, D.B., Pi-Sunyer, X. et al (2004). Clinical complications of obesity with specific focus on cardiovascular disease. Circulation, 2, 2952-2967.

Kobaissi, H.A., Weigensberg, M.J., Ball, G.D.C., Cruz, M.L., Shaibi, G.Q., \& Goran, M.I. (2004). Relation between Acanthosis Nigricans and insulin sensitivity in overweight Hispanic children at risk for type 2 diabetes. Diabetes Care, 27, 1412-1416.

Kong A.S., Williams R.L., Smith, M., Sussman, A.L., Skipper, B., His, A.C. et al. (2007). Acanthosis Nigricans and diabetes risk factors: Prevalence in young persons seen in South Western US primary care practices. Annals of Family Medicine, 5(3), 202-208.

Krosnick, A. (2000). The diabetes and overweight epidemic among the Pima Indians. New Jersey Medicine, 97, 31-37.

Kumanyika, S., Jeffrey, R.W., Morabia, A., Ritenbaugh, C., \& Antipatis, V.J. (2002). Obesity prevention: The case for action. International Journal of Obesity Related Metabolic Disorders, 26, 425-436.

Kwan, M., Woo, J., \& Kwok, C.Y.T. (2004). The standard oxygen consumption value equivalent to one metabolic equivalent $93.5 \mathrm{ml} / \mathrm{min} / \mathrm{kg}$ ) is not appropriate for elderly people. International Journal of Food Sciences and Nutrition, 55(3), 179-182.

Laasko, M. (1996). Insulin resistance and coronary heart disease. Current Opinion in Lipidology, 7(4), 217-226.

Laboratory Standardization Panel. Recommendations for improving cholesterol measurement. A report from the Laboratory Standardization Panel of the National Cholesterol Education Program. NIH Publication no. 90-2964. Bethseda, MD. NIH. 1990. 
Lalta, S. (1995). Review of health financing in Jamaica and a survey of the feasibility of National Health Insurance. Institute of Social and Economic Research, University of the West Indies, Mona, Kingston, Jamaica. A study funded by the USAID through LAC.

Leon, A.S. (1997). Physical activity and cardiovascular health: a national consensus. Human Kinetics: Champaign, IL. pp. 272.

Li, C., \& Ford, E.S. (2007). Is there a single underlying factor for the metabolic syndrome in adolescents? Diabetes Care, 30, 1555-1561.

Liu, S. (2002). Intake of refined carbohydrates and whole grain foods in relation to risk of type 2 diabetes mellitus and coronary heart disease. Journal of the American College of Nutrition, 21(4), 298-306.

Liu, S., Manson, J.E., Stampfer, M.J., Rexrode, K.M., Hu, FB. \& Rimm, E. B. et al. (2000). Whole grain consumption and risk of ischemic stroke in women: a prospective study. Journal of the American Medical Association, 284, 1534-1540.

Liu, S., Manson, J.E., Stampfer, M.J., Holmes, M.D., Hu, F.B., Hankinson, S.E., \& Willett, W.C. (2001). Dietary glycemic load assessed by food frequency questionnaire in relation to plasma high-density lipoprotein cholesterol and fasting triglycerides among postmenopausal women. American Journal of Clinical Nutrition, 73, 560-566.

Loke, A.Y. (2005). Dietary habits of patients with coronary atherosclerosis: case- control study. Journal of Advanced Nursing 52(2), 159-161.

Mahan, L.K., \& Escott-Stump, S. (1996). Krause's Food, Nutrition, and Diet Therapy. $9^{\text {th }}$ edition. W.B. Saunders Company.

Marcovecchio, M., Mohn, A., \& Chiarelli, F. (2005). Type 2 diabetes mellitus in children and adolescents. Journal of Endocrinology Investigation, 28(9), 853-863.

Martorell, R., Khan, L.K., Hughes, M.L., \& Grummer-Strawn LM. (1998). Obesity in Latin American women and children. American Society for Nutritional Sciences, 128 (9)1464-1473.

Mayer-Davis, E.J. (2000). Does diet play a role in the etiology of type 2 diabetes? Fats versus carbohydrates. Presented at the $60^{\text {th }}$ Scientific Sessions of the American Diabetes Association; June 10, 2000, San Antonio, Texas. 
McClain, M.R., Srinivasan, S.R., Chen, W., Steinmann, W.C., \& Berenson, G.S. (2000). Risk of type 2 diabetes mellitus in young adults from a biracial community: The Bogalusa Heart Study. Preventive Medicine, 31, 1-7.

McGillis-Bindler, R., \& Bruya, MA. (2005). Evidence for identifying children at risk for being overweight, cardiovascular disease, and type 2 diabetes in Primary Care. Journal of Pediatrics Health Care, 20(2), 82- 87.

McKeigue, P.M., Shah, B., \& Marmot, M.G. (1991). Relation of central obesity and insulin resistance with high diabetes prevalence and cardiovascular risk in South Asians. Lancet, 337, 382-386.

McKenzie, P.M., Shah, B., \& Marmot, M.G. (1991). Relations of central obesity and insulin resistance with high diabetes prevalence and cardiovascular risk in South Asians. Lancet, 337, 382-386.

McKeown, T. (1988). The origins of human disease. Oxford, Blackwell p 233.

McKnight-Menci, H., Sababu, S., \& Kelly S.D. (2005). The care of children and adolescents with type 2 diabetes. Journal of Pediatric Nursing, 20(2), 96-106.

Miller, E. (1986). Marginalization of the Jamaican Male._Institute of Social and Economic Studies, University of the West Indies, Mona, Jamaica.

Monsen, E. 1992. Research: Successful Approaches. The American Dietetic Association 216 West Jackson Boulevard, Suite 800, Chicago, Illinois 606066995.

Monteiro, C.A., Conde, W.L., Lu, B., \& Popkin, B.M. (2004). Obesity and inequities in health in the developing world. International Journal of Obesity, 28(9), 1181-1186.

Monteiro, C. A., Moura, E.C., Conde, W. L., \& Popkin, B. M. (2004). Socioeconomic status and obesity in adult populations of developing countries: A review. Bulletin, World Health Organization 82; 940-946.

Moreno, L.A., Pineda, I., Rodriguez, D., Fleta, J., Sarria, A., \& Bueno, M., (2002). Waist circumference for the screening of the metabolic syndrome in children. Acta Pediatric, 91(12), 1307-1312.

Moses, R.G., Calvert, D., \& Storlien, L.H. (1996). Evaluation of the Accutrend GCT with respect to triglyceride monitoring. Diabetes Care, 19, 1305-1306.

Murray, C. J., \& Lopez, A.D. (1994). Global and regional cause of-of-death patterns in 1980. Bulletin of World Health Organization, 72, 447-480. 
Nakamoto, M. (2006). H-B-A-1-C Diabetes Self-Management. http://www.DiabetesSelfmanagement.com Retrieved 6/20/2006.

National Cholesterol Education Program: Second Report of the Expert Panel on Blood Cholesterol in Children and Adolescents, NHLBI, USDHHS, NIH Publication, Bethseda, MD: NIH (1991).

National Center for Youth Development, Ministry of Education, Youth and Culture, Jamaica, (2002). Focus on Young Adults Program, Pathfinder International Policy II Project, Futures group International ALEPH, SA. pp 6-8.

National Cholesterol Education Program. Recommendations on lipoprotein measurement from the Working Group on Lipoprotein Measurement. NIH Publication N0. 95-3044. Bethseda, MD: National Heart, Lung, and Blood Institute. 1995.

National Cholesterol Education Program: Second Report of the Expert Panel on Detection, Evaluation and Treatment of High Blood Cholesterol in Adults (ATP-II). (1993). NIH Publication No. 93: 3095.

National High Blood Pressure Education Working Group on Hypertension Control in Children and Adolescents. (1996). Update on the 1987 Task Force Report on high blood pressure in children and adolescents. A Working Group Report from the National High Blood Pressure Education Program. Pediatrics, 98, 649-658.

National Institute of Diabetes and Digestive and Kidney Diseases (NIDDK). Statistics related to obesity and overweight [Internet]. [Bethseda (MD)]: NIH; 1996, July (NIH Publication No. 96-4158). Available from www.niddk.nih.gov/health/nutrit/pubs/staobes.htm Retrieved 7/21/2006.

Neilson, S.J., \& Popkin, BM. (2003) Patterns and trends in foods portion sizes, 1977 1998. Journal of the American Medical Association, 289(4), 450-453.

Nelson, JA., Carpenter, K., Chiasson MA. (2006). Diet, activity, and overweight among pre-school age children enrolled in the Special Supplemental Nutrition program for Women, Infants and Children (WIC). Public Health Research, Prevention and Policy, 3 (2), 1-12.

Nesmith, J.D. (2001). Type 2 diabetes mellitus in children and adolescents. Pediatrics in Review, 22, 147-152.

Nguyen, T.T., Keil, M.F., Russell, .D.L., et al. (2001). Relation of acanthosis nigricans to hyperinsulinemia and insulin sensitivity in overweight African American and white children. Journal of Pediatrics, 138, 474-480. 
Nguyen, VT., Larson, D.E., Johnson, R.K., \& Goran, M.I. (1996). Fat intake and adiposity in children of lean and obese parents. American Journal of Clinical Nutrition, 63, 507-513.

Nichols, J.H. (2006). What is the appropriate reference interval for glucose? Point of Care: The Journal of Near-Patient Testing \& Technology, 5(2), 49-51.

Norberg, M., Eriksson, J.W., \& Lindahl, B. et. al. (2006). A combination of HbA1c, fasting glucose and BMI is effective in screening for individuals at risk of future type 2 diabetes: OGTT is not needed. Journal of Internal Medicine, 260 (3), $263-271$.

Nugent, R. (2006). Obesity-related diseases creep up on developing countries. Population Reference Bureau. http://www.Prb.org/Template.cfm?Section=PRB\&template=/ContentManagement /ContentDisplay Retrieved 6/6/2006.

Nycocard Diabetes Screening. Williams Medical Supply. http://www.wmsplc.co.uk/Nycocard_Diabetes_Screening.html Retrieved 11/28/2006.

O’ Dea, K. White, N.G., \& Sinclair, A.J. (1998). An investigation of nutrition related risk factors in an isolated Aboriginal community in northern Australia: advantages of a traditionally-oriented lifestyle. Medical Journal of Australia, 148, 177-180.

Pan American Health Organization. (2008). PAHO representative highlights chilling effects of non-communicable diseases. Jamaica Information Service, http://www.jis.gov.jm Retrieved 02/01/2009.

Pan, QX., Liu, P, Wang, S.C., Pan, J.T., Sun, B.Y., Wu, X.Y. et al. (1991). The study of serum apoprotein levels as indicators for the severity of angiographically assessed coronary artery disease. American Journal of Clinical Pathology, 95, 597-600.

Pan, X.R., Li, G.W., Hu, Y.H. Wang, J.X., Yang, Y., An, Z. et al. (1997). Effects of diet and exercise in preventing NIDDM in people with impaired glucose tolerance. The Da Qing IGT and Diabetes Study. Diabetes Care, 20, 537-544.

Pan, Y., \& Pratt, C. (2008). Metabolic syndrome and its association with diet and physical activity in US adolescents. Journal of the American Dietetic Association, 108(2), 276-286.

Pate, R.R., Pratt, M., \& Blair, S.N. (1995). Physical activity and public health: A recommendation from the Centers of disease Control and Prevention and the 
American College of Sports Medicine. Journal of the American Medical Association, 341, 650-658.

Pereira, M.A. (2003). Types of carbohydrates and risk of cardiovascular disease. Journal of Women's Health, 12 (2), 115-122.

Pinhas-Hamiel, O., Dolan, M.N., \& Zeitler, P.S. (1997). Diabetic ketoacidosis among obese African American adolescent with NIDDM. Diabetes Care, 20, 484-486.

Ponder, S.W. Sullivan, S. \& McBath, G. (2000). Type 2 Diabetes Mellitus in teens. Diabetes Spectrum, 13(2), $95-117$.

Popkin, B.M. (2001). The nutrition transition and obesity in the Developing World. (Presented at the Symposium on Obesity in Developing Countries: Biological and Ecological Factors). American Society for Nutritional Sciences, 131(33), 871S$873 \mathrm{~S}$.

Popkin, BM. (1999). Urbanization, lifestyle changes and the nutrition transition. World Development, 27, 1905-1916.

Pratt, M., Marcera, C.A., \& Wang, G. (2000). Higher direct medical costs associated with physical inactivity. Physiology and Sports Medicine, 28, 63-70.

Prentice, A. (2006). The emerging epidemic of obesity in developing countries. International Journal of Epidemiology, 35, 93-99.

Ragoobirsingh, D., Lewis-Fuller, E, Morrison, E.Y. (1995). The Jamaican Diabetes Survey. Diabetes Care, 18(9), 1277-1279.

Ramachandran, A. (2004). Diabetes and Obesity- The Indian Triangle. Indian Journal of Medical Research. Indian Council of Medical Research. Nov 2004. http://www.findarticles.com/p/articles/mi_qa3867/is_200411/ai_n9466352/print. Retrieved 6/62006.

Ramachandran, A., Snehalatha, C., Viswanathan, V., Viswanathan, M., \& Haffner, SM. (1997). Risk of non-insulin dependent diabetes mellitus conferred by obesity and central adiposity in different ethnic groups: a comparative analysis between Asian Indians, Mexican Americans and Whites. Diabetes Research in Clinical Practice, 36, 121-125.

Ramachandran A., Snehalatha, C., Vintha, R. et al (2002). Prevalence of overweight in urban Indian adolescent school children. Diabetes Research and Clinical Practice, 57, 185-190. 
Ramachandran A., Snehalatha, C., Satyavani, K., Sivasankari, S., \& Vijay, V. (2003). Type 2 diabetes in Asian Indian urban children. Diabetes Care, 26, 1022-1025.

Reaven, GM. (1987). Do high carbohydrate diets prevent the development or attenuate the manifestations (or both) of syndrome X? A view point strongly against. Curr Opinion Lipidol, 8, 23-27.

Reaven, GM. (1988). Role of insulin resistance in human disease. Diabetes, 37, 1595-1607.

Reaven GM (2005). The metabolic syndrome: requiescat in pace. Clinical Chemistry, 51, 931-938.

Reinehr, T. (2005). Clinical Presentation of type 2 diabetes mellitus in children and adolescents. International Journal of Obesity, 29(2), S105-S110.

Riley, J.J., Dorosty, A.R., \& Emmett, P.M. (2000). The ALSPAC Study Team. Identification of the obese child: adequacy of the body mass index for clinical practice and epidemiology. International Journal of Obesity and Related Metabolic Disorders, 24, 1623-1627.

Rocthford, A. P., Rotchford, K.M., Machattie, T., \& Gills, V.G. (2002). Assessing diabetic control-reliability of methods available in resource poor settings. Diabetes UK Diabetic Medicine, 19, 195-200.

Rofling, C.L., Wiedmeyer, H., Little, R., Lee Crotz, V. \& Tennell, A. ( 2002). Biological variation of glycohemoglobin. Clinical Chemistry 48, 1116-1118.

Rosenbloom, A.l., Joe, J.R., Young, R.S., \& Winner, W.E. (1999). Emerging epidemic of type 2 diabetes in youth. Diabetes Care, 22, 345-354.

Rosenbloom, A.L., \& Silverstein, J.H. (2003). Type 2 diabetes in children and adolescents: A guide to diagnosis, epidemiology, pathogenesis, prevention, treatment. Alexandria, VA. American Diabetes Association.

Rubenstein, A.H. (2005). Obesity: a modern epidemic. Trans American Clinical Climatology Association, 116, 103-111.

Sackey, A.H., \& Jefferson I.G. (1996). Physical activity and glycemic control in children with diabetes mellitus. Diabetic Medicine, 13, 789-793.

Sacks, D.B., Bruns, D.E., Goldstein, DE., et al. (2002). Guidelines and recommendations for laboratory analysis in the diagnosis and management of diabetes mellitus. Clinical Chemistry, 48, 436-472. 
Sacks, D.B. (1999). Carbohydrates. In Burtis C, Ashwood E. eds. Tietz textbook of Clinical Chemistry, $3^{\text {rd }}$ ed. Philadelphia: WB Saunders, pp.750-808.

Sacks, D.B. (2005). Global harmonization of hemoglobin A1c. Clinical Chemistry, 51(4), 681-683.

Salmeron, J., Manson, J.E., Stampfer, MJ., Colditz, G.A., Wing, AL., \& Willet, WC. (1997). Dietary fiber, glycemic load, and risk of non-insulin dependent diabetes mellitus in women. Journal of the American Medical Association, 277, 472-477.

Sandbaek, A., Lauritzen, T., Borch-Johnsen, K., Mai, K, \& Christiansen, J.S. (2005). The comparison of venous plasma glucose and whole blood capillary glucose in diagnoses of type 2 diabetes: a population based screening study. Diabetic Medicine, 22, 1173-1177.

Sargeant, L.A., Boyne, M.S., Bennett, F.I., Forester, T.E., Cooper, R.S., \& Rainford, J. (2004). Impaired glucose regulation in adults in Jamaica: who should have the oral glucose tolerance test? Pan American Journal of Public Health, 16(1), $35-42$.

Schwartz, K.L., Monsur, J.C., Bartoces, M.G., West, P.A., \& Neale, A.V. (2005). Correlation of same-visit HbAlc test with laboratory -based measurements: A MetroNet Study. BMC Family Practice, http://www.biomedcentral.com/4712296/6/28 Retrieved July 16, 2007.

Schlosser, E. (2002). Fast Food Nation: The dark side of the all-American Meal.Harper Collins Publishers.

Schmitz, K.H., Jacobs, D.R., Hong, C-P., Steinberger, J., Moran, A., \& Sinaiko, A.R. (2002). Association of physical activity with insulin sensitivity in children. International Journal of Obesity, 26, 1310-1316.

Scott, C.R., Smith, J.M., Cradock, M.M., \& Pihoker, C. (1997). Characteristics of youth onset non-insulin dependent diabetes mellitus at diagnosis. Pediatrics, 100, 84-91.

Seidell, J.C., Han, T.S., Feskens, J.M., \& Lean, M.E. (1997). Narrow Hips and broad waist circumferences independently contribute to increased risk of noninsulin-dependent diabetes mellitus. Journal of Internal Medicine, 242, 401-406.

Seidell, J.C., Perusse, L., Jean-Pierre, D., \& Bouchard, C. (2001). Waist and hip circumferences have independent and opposite effects on cardiovascular disease risk factors: the Quebec family Study. American Journal of Clinical Nutrition, 74, 315-321. 
Shankar, A., Klein, R, Klein, B.E.K., \& Moss, S.E. (2007). Association between glcycosylated hemoglobin level and cardiovascular and all-cause mortality in type 1 diabetes. American Journal of Epidemiology, 166, 393-402.

Sicard, D.A. \& Taylor, J.R. (2005). Comparison of Point-of-care HbA1c Test versus Standardized laboratory Testing. The Annals of Pharmacotherapy. 39(6), 10241028.

Sicree, S.J., \& Zimmet, P. (2003). The global burden of diabetes. In: Diabetes Atlas, $2^{\text {nd }}$ edition. Brussels: International Diabetes Federation, pp 15-71.

Siepel, M.M.O. (2005). Social burden of obesity on US adults. Journal of Health and Social Policy, 20(2), 1-14.

Simon, D., Coignet, M.C., Thibult, N., Senan, C., \& Eschwege, E. (1985). Comparison of glycosylated hemoglobin and fasting plasma glucose with two hour post load glucose in the detection of diabetes mellitus. American Journal of Epidemiology, 122(4), 589-593.

Singh, R.B., Dubnov, G., Niaz, M.A. et al. (2002). Effects of an Indo- Mediterranean diet on progression of coronary artery disease in high risk patients (IndoMediterranean Diet heart Study): a randomized single-blind trial. Lancet, 360 (9344), 1455-1461.

Singh, R., Shaw, J., \& Zimmet, P. (2004). Epidemiology of childhood type II diabetes in the developing world. Pediatric Diabetes, 5, 154-168.

Sinha, R., Fisch, G., Teague, B., Tamborlane, W.V., Banyas, B. et al. (2002). Prevalence of impaired glucose tolerance among children and adolescence with marked obesity. New England Journal of Medicine, 346, 802-810.

Slein, M.W. (1963). Methods of Enzyme Analysis, (Bergmeyer, H.U.), New York, Academic Press.

Smickklas-Wright, H., Mitchell, D.C., Mickle, S.J., Goldman, J.D., \& Cook, A. (2003). Foods commonly eaten in the United states, 1989-1991 and 1994-1998. Journal of the American Dietetic Association, 103(1), 41-47.

Snehalatha, C., Viswanathan, V., \& Ramachandran A. ((2003). Cutoff values for normal anthropometric variables in Asian Indian adults. Diabetes Care, 26, 1380- 1384.

Sorof, J.M., Poffenbarger, T., Franco, K., Bernard, L., \& Portman, R.J. (2002). Isolated systolic hypertension, obesity, and hyperkinetic hemodynamic states in children. Journal of Pediatrics, 140, 660-666. 
Soljie, A.N., \& Thuen, F. (1995). School journeys and leisure activities in rural and urban adolescents in Norway. Health Promotion International, 17(1), 21-30.

Statistical Institute of Jamaica (STATIN). (2001). http://www.statinja.com/images/census_pyramid_2001.jpg Retrieved 5/30/2006.

Steinberger, J., \& Daniels, R.(2003). Obesity, insulin resistance, diabetes, and cardiovascular risk in children. An American Heart Association scientific statement from the Atherosclerosis, Hypertension and Obesity in the Young Committee (Council on Cardiovascular Disease in the Young) and the Diabetes Committee (Council on Nutrition, Physical Activity, and Metabolism). Circulation, 107, 1448-1453.

Steinberger, J., Moran, A., Hong, C.P., Jacobs, D.R. Jnr, \& Sinaiko, A.R. (2001). Adiposity in childhood predicts obesity and insulin resistance in young adulthood. Journal of Pediatrics, 138, 469-473.

Stern, M.P. (1996). Do non-insulin-dependent diabetes mellitus and cardiovascular disease share common antecedents? Annals of Internal Medicine, 124(1PT 2), $110-116$.

Stuart, C.A., Driscoll, M.S., Lindquist, K.F., Gilkison, C.R., Shaheb, S., \& Smith, M.M. (1998). Acanthosis nigricans. Journal of Basic Clinical Physiology and Pharmacology, 9, 407-418.

Sylvan, H,F. Pokorny, M,E., \& English, S,M. (1995). Accuracy of finger prick glucose values in shock patients. American Journal of Critical Care, 4, 44-48.

Tajima, N. (2002). Type 2 diabetes in children and adolescents in Japan. International Diabetes Monitor, 14, 1-5.

Tamborlane, W. (2005). Comparison of finger stick hemoglobin Alc levels assayed by DCA 2000 with the DCCT/EDIC central laboratory assay: results of a Diabetes Research in Children Network (DirectNet) Study. Pediatric Diabetes, 6, 13-16.

Taylor, J.R., \& Lopez, L.M. (2004). Cholesterol: Point-of-Care Testing. The Annals of Pharmacotherapy, 38, 1252-1257.

The Caribbean Commission on Health and Development (CCHD) 2005 Report . http://www.jamaica-gleaner.com/gleaner/20070829/carib/carib4.html Retrieved 05/22/2009. 
The Expert Committee on the Diagnosis and Classification of Diabetes Mellitus. Report of the Expert Committee on the Diagnosis and Classification of Diabetes Mellitus. (1997). Diabetes Care, 20, 1183-1197.

Theintz, G. (2005). From Obesity to type 2 diabetes in children and adolescents. Review of Medical Science, 1(7), 477-480.

Thomas, A.G, Gough, J.E., Benson, N, et al. (1994). Accuracy of glucose determination in patient receiving CPR. South Medical Journal, 87, 1072-1075.

Tripathy, D., Almgren, P., Tuomi, T. \& Groop, L. (2004). Contribution of insulinstimulated glucose uptake and basal hepatic insulin sensitivity to surrogate measures of insulin sensitivity. Diabetes Care, 27 (9), 2204-2210.

Trowell, H.C., \& Burkett, D.P. (1981). Western diseases, their emergence and prevention. Cambridge, MA: Harvard University Press p 456.

Tucker, L.A. (1998). Obesity and absenteeism: An epidemiological study of employees. Journal of Health Promotion, 12(3), 202-207.

Tuomilehto, J., Lindstrom, J., \& Ericksson, J.G. et al. (2001). Prevention of type 2 diabetes mellitus by changes in lifestyle among subjects with impaired glucose tolerance. New England Journal of Medicine, 344, 1343-1350.

Vikram, N.K., Tandon, N., Misra, A., Srivastavat, M.C., Pandey, R.M., Mithal, A., et al. (2005). Correlates of Type 2 diabetes mellitus in children, adolescence and young adults in North India: a multi-site collaborative case-control study. Diabetic Medicine, 23, 293-298.

UNICEF. (1995). Monitoring progress toward the goals of the World Food Summit for children: A practical handbook for multiple indicator surveys. New York.

U.S. Department of Agriculture, Agricultural Research Service, 2005. USDA National Nutrient Database for Standard Reference, Release 18. Nutrient Data Laboratory Home Page, http://www.ars.usda.gov/ba/bhnrc/ndl

U.S. Department of Agriculture, Agricultural Research Service. 2007. USDA National Nutrient Database for Standard Reference, Release 20. Nutrient Data Laboratory Home Page, http://www.ars.usda.gov/nutrientdata

U.S. Department of Agriculture and U.S. Department of Health and Human Services. Dietary Reference Intakes for Energy, Carbohydrates, Fiber, Fat, Fatty Acids, Cholesterol, and Protein. (2002). 
University of Southern California School of Medicine (2007).

http://www.healthcalculators.org/calculators/waist_hip.asp Retrieved 06/29/2006.

Wang, X.L., Tam, C., McCredie, R.M., \& Wilcken, D.E.L. (1994). Determinants of severity of coronary artery disease in Australian men and women. Circulation, 89, 1974-1981.

Wang, C., Chung, M., Lichtenstein, A., Balk, E., Kupelnick, B., DeVine, D., Lawrence, A., \& Lau, J. (2004). Effects of omega-3 fatty acids on cardiovascular disease. Evidence Report/Technology Assessment No. 94 (Prepared by Tufts-new England Medical center Evidence-based Practice Center, under Contract No. 29002-0022). AHRQ Publication No.04-E009-2. Rockville, MD: Agency for Healthcare Research and Quality. March 2004.

Warnick, G.R., Leary, E.T., Ammirati, E.B., \& Allen, M.P. (1994). Cholesterol in fingerstick capillary specimens can be equivalent to conventional venous measurements. Archives of Pathology Laboratory Medicine, 118, 1110-1114.

Wei, J.N., Sung, F.C., Lin, C.C., Chang, C.C., Chuang, L.M. (2003). National surveillance for type 2 diabetes mellitus in Taiwanese children. Journal of the American Medical Association, 290, 1345-1350.

Weis, R. (2005). The metabolic consequences of childhood obesity. Best Practice and Research Clinical Endocrinology \& Metabolism, 19 (3), 405-419.

Welk, G.J. \& Wood, K. (2000). Physical activity assessments in physical education: A practical review of instruments and their use in the curriculum. JOPERD 71 (1), 30-40.

Whitaker, R.C., Wright, J.A., Seidel, K.D., \& Dietz, W.H. (1997). Predicting obesity in young adulthood from childhood and parental obesity. New England Journal of Medicine, 37(13), 869-873.

Wild, S., Roglic, G., Green, A., Sicree, R., \& King, H. (2004). Global prevalence of diabetes: estimates for the year 2000 and projections for 2030. Diabetes Care, 27, 1047-1053.

Williams, CJ., Hayman, L.L., Daniels, S.R., Robinson, T.N., Steinberger, J., Paridon, S. et al. (2002). Cardiovascular health in childhood: A statement for health professionals from the Committee on Atherosclerosis, Hypertension, and Obesity, in the Young (AHOY) of the Council on Cardiovascular Disease in the Young, American Health Association. Circulation, 106, 143-160. 
Willet, W.C., Manson, J.E., Stampfer, M.J., Colditz, G.A., Rosner, B., Speizer, FE., \& Hennekens, CH. (1995). Weight, weight change, and coronary heart disease in women. Risk within the "normal" weight range. Journal of the American Medical Association, 273(6), 461-465.

Windler, E. (2005). What is the consequence of an abnormal lipid profile in patients with type 2 diabetes or the metabolic syndrome? Atherosclerosis Supplements, 6, $11-14$.

Wolf, A.M., Colditz, G.A. (1998). Current estimates of the economic cost of obesity in the United States. Obesity Research, 6(2), 97-106.

Wolf, A. (1998). What is the economic cost for treating obesity? Obesity Research, 6(SI), 2S-7S.

World Health Organization. (2003). Global strategy on diet, physical activity and health: European regional consultation. Report on the Consultation, Copenhagen. EUR/HQ/03/905732. http://www.euro.who.int/Document/E81281.pdf Accessed January, 6, 2008.

Wyatt, S.B., Winter, K.P. \& Dubbert, D.M. (2006). Overweight and Obesity: prevalence, consequences, and causes of a growing public health problem. American Journal of Medical Science, Apr 331 (4), 166-174.

Yao, M., \& Roberts, S.B., (2001). Dietary energy density and weight regulation. Nutrition Review, 59, 247-258. 
APPENDICES 


\section{APPENDIX AI \\ CONSENT/ASSENT FORM}


CONSENT/ASSENT TO PARTICIPATE IN A RESEARCH STUDY

Title: Risk Factors of Type 2 Diabetes and Cardiovascular Diseases Among Jamaican Adolescents.

We are requesting your consent and your son/daughter's assent to be in a research study. The investigators of this study are Dr Fatma Huffman, Professor of Florida International University, and Sheila Barrett, doctoral student. The study will evaluate the risk factors that could cause your child to develop type 2 diabetes and cardiovascular diseases later in life. The study will involve screening approximately 360 other adolescents aged 14-19 years who will be selected from ten high schools in five different parishes in Jamaica for risk factors of type 2 diabetes and cardiovascular diseases. The entire study may take up to two hours of your child's time.

\section{Procedure:}

If you decide for your child to be a part of the study he/she will be asked to;

- Complete a 12 hour fast before the day of data collection and report to the sick bay upon arrival at school where he/she will undergo a physical examination by the school nurse who will measure height, weight, body fat percentage, and look for physical signs of Acanthosis Nigricans (a visible dark mark around the neckline which suggests the person may have diabetes).

- Report to the mobile unit of the Heart Foundation of Jamaica on location at the sehool to have his/her blood tested using finger prick method. A phlebotomist (an expert in drawing blood) will take a small amount of blood by pricking his/her finger. The blood will be used to determine his/her fasting plasma glucose. $\mathrm{HbAlc}$, and total cholesterol.

- Report to the Home Economics center in his/her school where he/she will be given a small breakfast after completing the blood test to prevent hypoglycemic event.

- Complete a questionnaire on past health history, family history of obesity, diabetes and cardiovascular diseases and a physical activity check list.

- Tell the researcher all the food and drink he/she consumed for the past 24 hours to assess hisher nutrient intake.

- After completing this blood tests and all questionnaires, he/she will be given a monetary incentive of US\$5.00 for participating in the study.

\section{Possible Risks}

The risks associated with this study are minimal. Your child might feel a little discomfort by finger pricking blood collection. With the right procedures for collecting blood, risks of infections are not likely. If any infection occurs, you should report to your child's pediatrician. Your child may also feel some discomfort by revealing information on how much food he/she eats and his/her physical activity levels. Some of the questions may be embarrassing to him/her. 
Benefits:

By participating in this study, you and your child will learn about the risk factors of type 2 diabetes and cardiovascular diseases. You and your child will receive the results of his/her blood test, and analysis and calculation of BMI. The research will give us information about Jamaican adolescents who may be at risk to type 2 diabetes and cardiovascular diseases and help us in planning preventive programs that can be made possible through the schools. There is no cost to you and your child to participate in the study.

Right to withdraw from the study:

The participation in the study is voluntary and you have the right to withdraw your child's participation from the study at any time.

\section{Confidentiality:}

To protect your child's privacy, all information gathered from him/her will be kept confidential. The information will be identified by a personal identification number (PIN) which will be assigned to him/her by the researcher. No one except Dr Huffman and Miss Barrett will know the name associated with his/her number. The data collected in this research will not be shared with anyone not directly related to the study unless required by law. The results will be presented as a group in all publications and at the general forum and conferences.

If you or your child would like to know more about the research, you can contact Dr Huffman at (305) 348-3788 or Miss Sheila Barrett at (305)408-1890. If either of you feel you were mistreated or you have questions about being in the study you may contact Dr. Patricia Price, the Chairperson of the Florida International University Institutional Review Board, at (305) 348 2618 or $(305) 348-2494$.

If you have had all your questions answered to your liking and you would like to be in the study, sign below.

(Print Teens Name)

Signature of Parent/Guardian

Date

If you have had all your questions answered to your liking and you would like to be in the study sign below.

Signature of Teen

Date

I have explained the research procedure, subject rights and answered questions asked by the participant. I have offered him/her a copy of this informed consent form.

Signature of Witness

Date 


\title{
APPENDIX AII
}

\section{IRB Approval Letter- Main Study}

\section{MEMORANDUM}

\author{
To: \\ Sheila Barrett \\ CC: \\ Dr. Fatma Huffman \\ File \\ From: \\ Chris Grayson, CIM, Asst. Director of Research Compliance \\ Date: \\ August 30, 2007 \\ Proposal Title: Risk Factors Of Type 2 Diabetes And Cardiovascular Diseases Among \\ Jamaican Adolescents.
}

Approval \# 062807-01

The Institutional Review Board of Florida International University has approved your study for the use of human subjects. Your IRB approval date is June 28, 2007 and this approval will expire on June 28,2008 . As a requirement of IRB approval you are required to:

1) Provide immediate written notification to the IRB of:

- Any additions to, or changes in the procedures involving human subjects,

- Every serious or unusual or unanticipated adverse event as well as problems with the rights or welfare of the human subjects. Confirmation of receipt of serious AE reports must be made with the IRB office.

2) Utilize copies of the date stamped consent document(s) for the recruitment of subjects and receive annual renewal of consent documents.

3) Receive annual review and re-approval prior to your expiration date.

Special Conditions: N/A

Please note your approval number is indicated above. For further information, you may contact the IRB Coordinator by email at irbiacuc@fiu.edu or visit the OSRA - Human Subjects website at www.osra. fiu.edu. 


\title{
APPENDIX AIII
}

\section{IRB Renewal Letter}

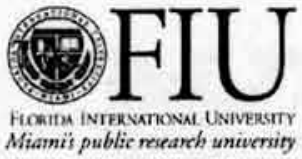

Office of Research Integrity

Research Compliance, MARC 430

\section{MEMORANDUM}

\author{
To: \\ Sheila Barrett \\ CC: \\ Dr. Fatma Huffman \\ File \\ From: \\ Chris Grayson, CIM, Asst. Director of Research Compliance \\ Date: \\ June 17, 2008 \\ Proposal Title: Risk Factors Of Type 2 Diabetes And Cardiovascular Diseases Among \\ Jamaican Adolescents.
}

Approval \# 062807-01

The Institutional Review Board of Florida International University has re-approved your study for the use of human subjects. Your IRB approval date is June 16, 2008 and this approval will expire on June 16, 2009. As a requirement of IRB approval you are required to:

1) Provide immediate written notification to the IRB of:

- Any additions to, or changes in the procedures involving human subjects.

- Every serious or unusual or unanticipated adverse event as well as problems with the rights or welfare of the human subjects. Confirmation of receipt of serious AE reports must be made with the IRB office.

2) Utilize copies of the date stamped consent document(s) for the recruitment of subjects and receive annual renewal of consent documents.

3) Receive annual review and re-approval prior to your expiration date.

Special Conditions: N/A

Please note your approval number is indicated above. For further information, you may contact the IRB Coordinator by email at irbiacucafiu.edu or visit the OSRA - Human Subjects website at www.osra. fiu.edu. 


\section{APPENDIX BI}

\section{Permission Letter- Ministry of Education, Youth and Culture, Jamaica}

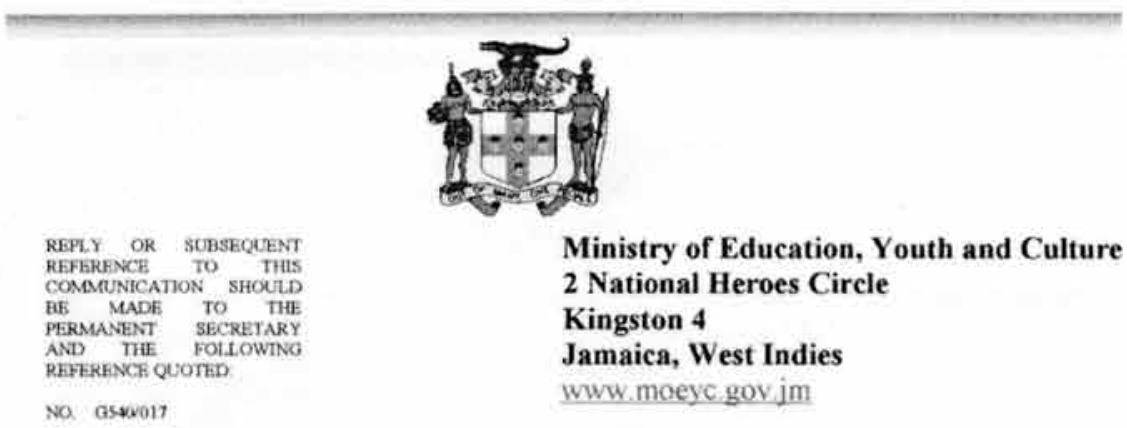

September 7, 2006

Ms Sheila Barrett

Graduate Student

Florida International University

Department of Dietetics and Nutrition

University Park, HLS 450

11200 S.W. $8^{\text {th }}$ Street

Miami, FL 33199

\section{Dear Ms Barrett}

Reference is made to your letter of September 7, 2006 in which you sought permission to conduct a study, which involves screening children ages 14-19 years for risk factors of Type 2 Diabetes and Cardiovascular Diseases in selected schools drawn from Administrative Regions One and Six.

Kindly be advised that the Ministry has granted permission for you to proceed with this research undertaking with the understanding that confidentiality and anonymity be maintained. However where medical procedures are concerned you need to get the approval from the Ministry of Health and consent from parents

Best wishes

Sincerely yours

Jaspef Lawrence

Chief Education Officer (actg)

cc: Professor Fatma G. Huffman Ph.D., RD 


\title{
APPENDIX BII
}

\section{Permission Letter- Ministry of Education, Youth and Culture, Jamaica}

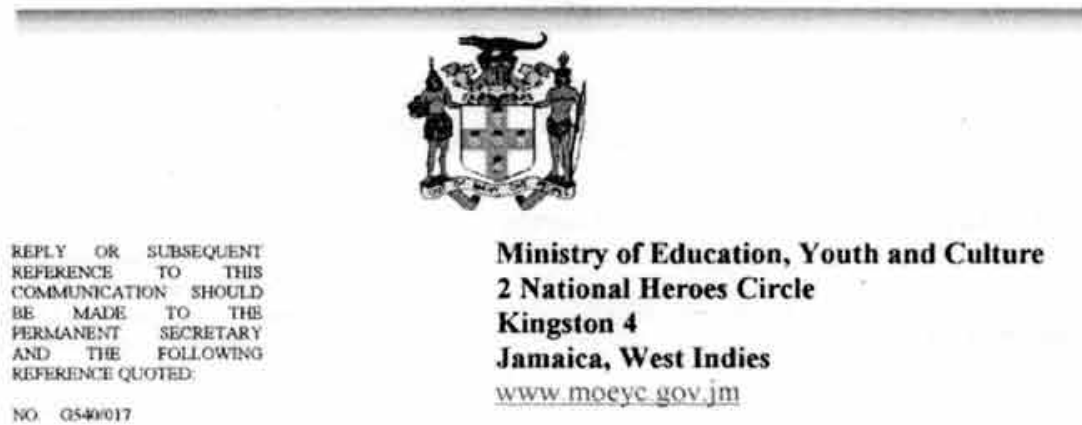

September 7, 2006

Dear Principal

This serves to introduce Ms. Sheila Barrett a graduate student who is reading for the Doctor of Philosophy Degree in Dietetics and Nutrition, Department of Dietetics and Nutrition, Florida International University. She wishes to conduct a study which involves screening children ages 14-19 years for risk factors of Type 2 Diabetes and Cardiovascular Diseases and as such would also be seeking to involve Home Economics and Science Teachers, School Nurse and Guidance Counsellors (details of the study is attached).

Kindly be advised that the Ministry has granted approval for her to proceed with this assignment and your school was selected to be a part of the sample. With this in mind, your cooperation is being solicited in facilitating her as she embarks on this undertaking.

Grateful for your usual support.

Sincerely yours

\author{
Jasper Lawrence \\ Chief Education Officer (actg.) \\ cc: Mr. Errol Levy, Regional Director, Region 6 \\ Ms. Vilma Blair, Regional Director (actg.), Region 1 \\ Ms. Beryl Jengelley, Regional Director, Region 2
}




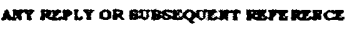
To this cowaronch Trom should ax

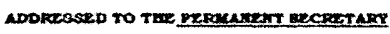

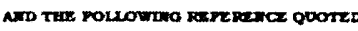

Ref : S\&R/R/10

2007 January 04

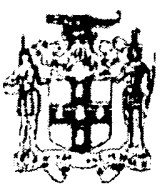

MINISTRY OF HEALTH

STANDARDS \& REGULATION DIVISION OCEANA COMPLEX

2-4 KING STREET,

KINGSTON, JAMAICA

Tel: (876) 967-11009/948-4106 Fax: 967-1629

Professor Fatma G. Huffmon Ph.D., RD

Florida International University

Department of Dietetics and Nutrition

University Park, HLS 450

11200 S. W. $8^{\text {th }}$ Street

Miami, Florida 33199

USA

Dear Professor Huffman

\section{Permission for Doctoral Study to be conducted in Selected Schools in Jamalca}

Reference is made to a request received from Ms. Shelia Barrett, doctoral student at the Florida International University to conduct her Dissertation Research in adolescents aged 14-19 years for risk factors for Type 2 Diabetes and Cardiovascular Disease in selected schools in Jamaica.

This is to inform you that permission is hereby granted for the study to be conducted in the schools indicated.

Ms. Barrett is to note that permission is granted on condition of adherence of specifications of the Ministries of Education and Health. Subsequent to this permission being granted, Ms. Barrett is expected to submit details of the intended sludy including the appropriate consent forms for use in the study.

Yours sincerely

Princess Thomas Osboume (Mrs.)

Director, Standards \& Regulation Division

Copy: Ms. Sheila Barretł. Doctoral Student 


\section{Permission Letter- Principals}

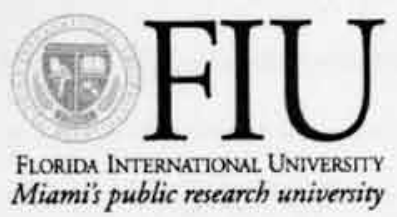

August 26, 2007

The Principal,

\section{Dear Sir/Madam,}

Your school was randomly selected to participate in a research study conducted by doctoral student, Sheila Barrett and Dr Fatma G. Huffman (major professor) of Florida International University. We are seeking your permission to conduct the research study in your institution. This study is in partial fulfillment for a $\mathrm{PhD}$ in Dietetics and Nutrition at Florida International University for Miss Barrett.

The study involves screening children ages $14-19$ years for risk factors of type 2 diabetes and cardiovascular diseases. Recent research has shown an increasing trend towards type II diabetes and cardiovascular diseases in younger cohorts of children and adolescents.

If school permission is granted, we will seek further consent from parents, and students to participate in this study. The study will be conducted in two phases; 1) an initial screening for risk factors of type 2 diabetes and cardiovascular disease, and 2) a follow - up study for select students found to be at risk for these health conditions. In phase one students will be asked to a) undergo a physical examination by a nurse, b) have their blood tested using finger prick method, c) keep a 3 day record of all food and drink consumed, d) complete a demographic questionnaire, and e) complete a physical activity checklist. Students will be paid US\$5.00 on completion of all five research activities stated above. Selected students will be invited back for further testing using venipuncture in phase two of the study.

We are also requesting the support of the Home Economics/Science Teacher/School Nurse / Guidance Counselor in your school to help with data collection for which they will be reimbursed for each student they recruit and who successfully complete all five research activities for the study. Only one teacher per school will be recruited.

I am therefore asking your permission to conduet this study in your school and to suggest the name of a teacher who is willing to help with the data collection. Please give your response by checking the attached form and return in the self-addressed, stamped envelope. Your prompt response will be appreciated. Thank you.

Yours truly,

Sheila C. Barrett

Graduate Student
Fatma G. Huffman Ph. D.

(Major professor)

Rober R. Stempel School of Public Health

Department in Dietetics and Nutrition

11200 S.W. 8th Steet, HLS-1 450 - Miami, FL 33199 • Tel: (305) 348-2878 • Fax: (305) 348.1996 
RESPONSE TO REQUEST

1 give permission to allow the researcher to conduct this study in my sehool

DYes

$\square \mathrm{No}$

Signature

Date

I recommend the following teacher to help with data collection

Name of teacher

Telephone

Address:

Email Address: 


\section{APPENDIX E}

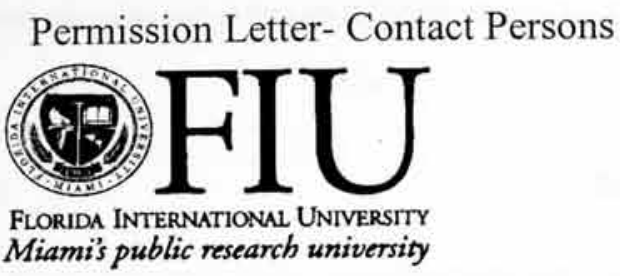

August 26, 2007

\section{Dear Sir/Madam,}

Your school was randomly selected to participate in a research study conducted by Sheila Barrett and Dr Fatma G. Huffman of Florida International University. This study is in partial fulfillment for a Ph.D. in Dietetics and Nutrition at Florida International University for Ms Barrett.

The study involves screening children ages 14-19 years for risk factors of type 2 diabetes and cardiovascular diseases. If school permission is granted, we will seek further consent from parents, and students to participate in this study. Approximately 360 students from ten high schools in Jamaica will be asked to participate in the study. Students will be randomly selected from grades 9-11 and will include twelve students from each grade level. 60 students from the original sample will be asked to undergo further testing. Recent research has shown an increasing trend towards type 2 diabetes and cardiovascular diseases in young children and adolescents.

Students will be asked to a) undergo a physical examination by a nurse, b) have their blood tested using finger prick method, c) tell the researcher all food and drink consumed for the past 24 hours, d) complete a demographic questionnaire, and e) complete a physical activity checklist. Students will be paid US\$5.00 on completion of all five research activities stated above. Selected students will be invited back for further testing using venipuncture.

You were recommended by your principal to assist in conducting this study. Should you agree to help with this study you will be required to 1) help in recruiting a total of 36 students from grades 9-11,2) distribute letters of consent to parents, and 3) help in organizing students for blood tests on data collection days. On completion of these research activities you will be given a small stipend for each student who successfully completed all research activities.

Please indicate your consent by signing the attached form and return in the selfaddressed, stamped envelope. Your prompt response will be appreciated. Thank you.

Yours truly,

Sheila C. Barrett

(Graduate Student)
Fatma G. Huffman Ph.D., RD.

Major Professor) 


\section{RESPONSE TO REQUSET}

I agree to assist with the collection of data for this study in my school
$\square$ Yes
$\square$ No

Name of Teacher

Date

Telephone

Address:

Email Address: 
APPENDIX F

\section{DEMOGRAPHIC QUESTIONNAIRE FOR ADOLESCENTS}

TO BE COMPLETED BY RESEARCHER AND /OR CONTACT PERSON

1. Name of School:

2. Place of residence:

1. Rural

2. Urban

3. Student:

ID \#:

4. Date of Birth:

5. Age:

6. Gender: $\quad 1 . \square \quad$ Male

2. Female

7. What is your grade level in school?

1. $\square$ Grade 9

2. $\square$ Grade 10

3. $\square$ Grade 11

8. Ethnicity:
1. $\square$ Black
2. $\square$ Indian
3. $\square$ Chinese
4. $]$ Caucasian

5. $\square$ Mixed (Please state)

9. Do you consider yourself a physically active person? $\quad 1 . \square$ Yes $\quad 2 . \square$ No

9b. Approximately, how many hours per week do you spend doing the following?

Sedentary activities? Moderate activities? Vigorous activities?

10. Approximately, how many hours per week do you watch TV?

11. Approximately, how many hours per week do you spend on the computer or playing video games?

12. Do you consider your weight to be
1. $\square$ Below Normal
2. $\square$ Normal
3. $\square$ Above Normal

13. How do you rate your food habits?
1. $\square$ Poor
2. $\square$ Fair
3. $\square$ Good
4. $\square$ Excellent 
14. Family History of Obesity:

Circle the number from the diagram below that best describes these persons:

1. Mother

12234556789

3. Brother

12234556788
2. Father

1234566789

4. Sister 5. Me
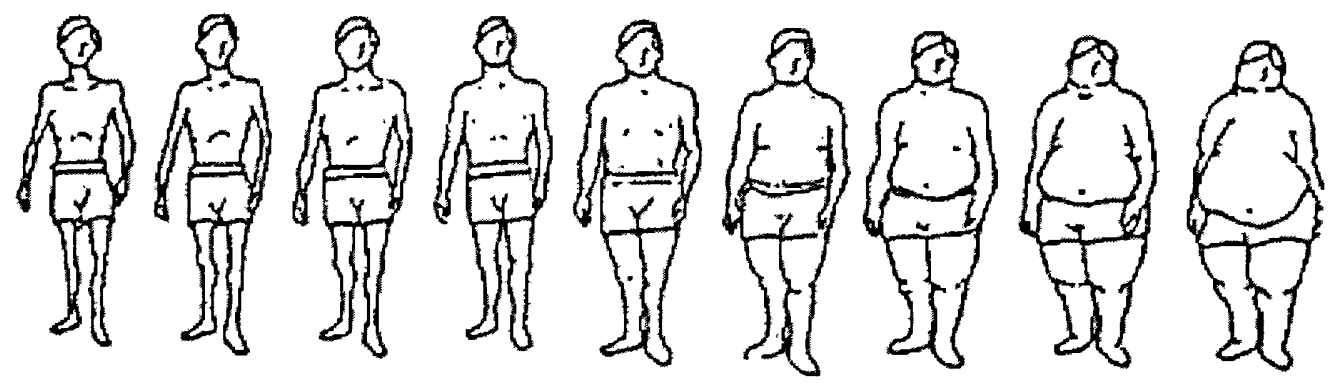

1

2

3

45

6

7

8

9
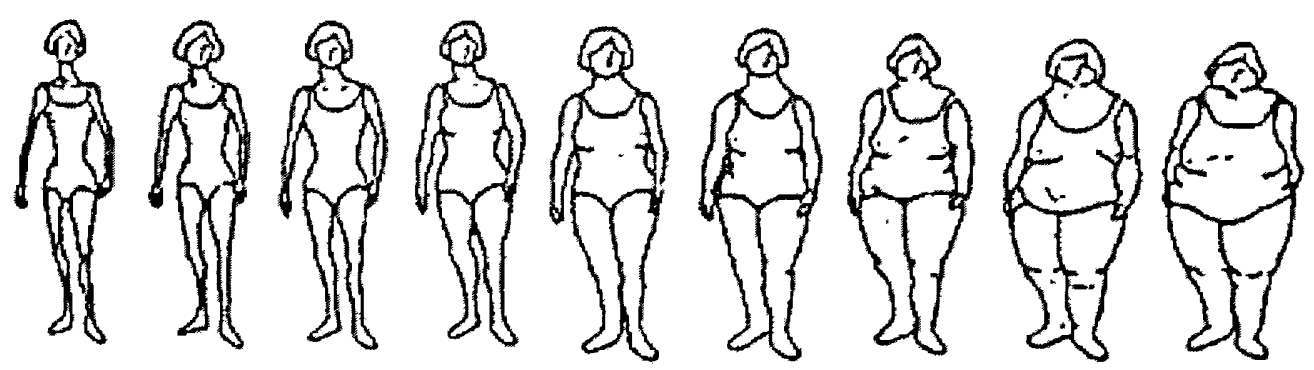

$14 \mathrm{~b}$. Which of the above figures would you want to look like?

1233456789

\section{Family History of Diabetes}

15a. Have you ever been diagnosed with diabetes? $1 . \square$ Yes

2. $\square$ No

$15 \mathrm{~b}$. Does anyone in your family have diabetes?

$15 \mathrm{c}$. If yes, indicate who;
1. $\square$ Mother only
2. $\square$ Father only
3. $\square$ Both parents
4. $\square$ grandparents
5. $\square$ Aunt
6. $\square$ Uncle

\section{7. $\square$ Cousin}

16. Family History of Heart Diseases

16a. Have anyone in your family been diagnosed by a nurse or doctor for having angina or stroke?

1. $\square$ Yes

2. $\square$ No

16b. If yes, indicate who; 
1. $\square$ Mother only

4. $\square$ Grandparents
2. $\square$ Father only

5. $\square$ Uncle
3. $\square$ Both parents

6. $\square$ Aunt

\section{7. $\square$ Cousin}

16. Mother's Occupation:

Father's Occupation:

17. Parents' Educational Background

$17 \mathrm{a}$. What is the highest level of education for your father?

1. $\square$ Primary

2. $\square$ Secondary

3. $\square$ Diploma

4. $\square$ Bachelors Degree

5. $\square$ Masters degree

6. $\square$ More than a Masters degree

$17 \mathrm{~b}$. What is the highest level of education for your mother?

1. $\square$ Primary

2. $\square$ Secondary

3. $\square$ Diploma

4. $\square$ Bachelors Degree

5. $\square$ Masters degree

6. $\square$ More than a Masters degree

18. Average Household Income (per month) J\$

1. $\square 10,000.00$ or less

2. $\square 10,00120,000$

3. $\square 20,001-30,000$

4. $\square 30,001-40,000$

5. $\square 40,001-50,000$

6. $\square$ Over 50,000

19. With whom do you live? (Check all that apply).
1. $\square$ Mother and father
2. $\square$ Mother only
3. $\square$ Father only
4. Grandparents
5. $\square$ Sister
6. $\square$ Brother
7. $\square$ Aunt
8. $\square$ Uncle
9. $\square$ Other (please state) 


\section{APPENDIX G}

\section{Contract Agreement- Heart Foundation of Jamaica}

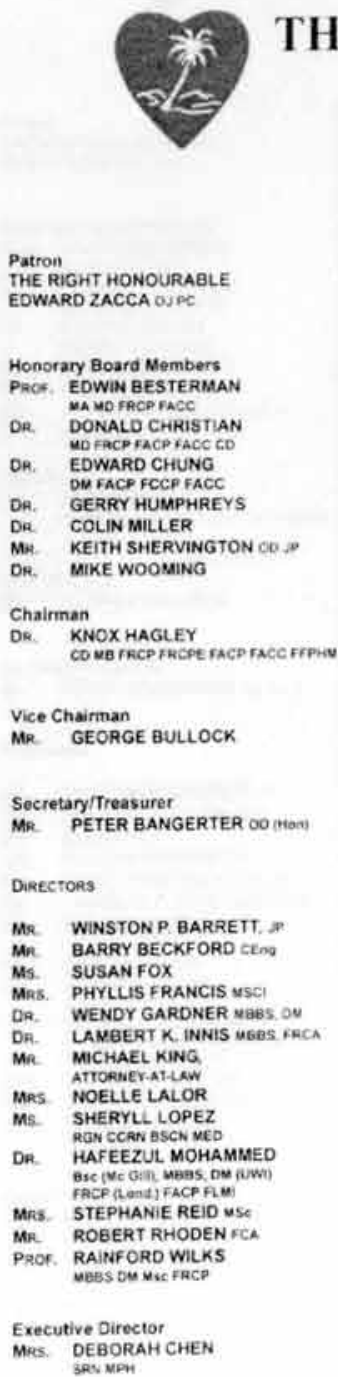

Patron
THE RIGHT HONOURABLE EDWARD ZACCA OJPC

September 7, 2006

Florida International University

Department of Dietetics \& Nutrition

University Park, HLS 450

11200 S.W. $8^{\text {th }}$ Street

Miami, FL 33199

US A

Dear Sir/Madam

The Heart Foundation of Jamaica has agreed to support Miss Sheila Barrett, doctoral student of Florida International University, in conducting her Dissertation Research.

The study involves testing Jamaican adolescents, aged 14-19 years for risk factors of Type II Diabetes and Cardiovascular Disease. The Heart Foundation of Jamaica, through the use of its Mobile Unit, will facilitate this data collection in ten (10) selected high schools by conducting the followings tests:

- Fasting Blood Glucose

- Total Cholesterol

- Blood Pressure and

- Body Mass Index

Attached is a copy of the proforma invoice, detailing the cost of these services.

Yours sincerely

THE. HEART FOUNDATION OF JAMAICA

Novelette Dónn, BBA (Hons.), MSc.

Administrative Manager 

30 BEECHWOOD AVENUE, PO. BOX 338. KINGSTON 5 TEL: (876) 926-4378, 926-6492, 929.3195 FAX: (876) 754.6441 e-mail: hfj@mail.infochan.com Website: www.heartfoundation.org.jm

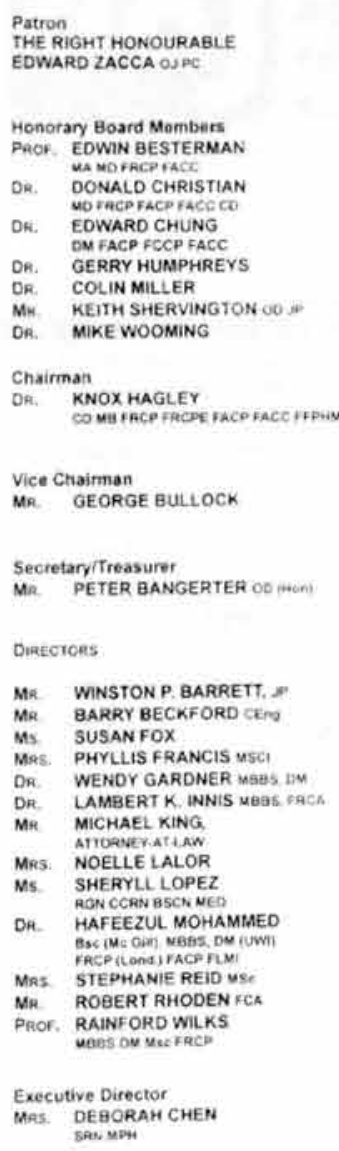

RE: BMI \& Blood Pressure checks, Total Cholesterol and Blood sugar Tests done in 10 schools shown below

BMI/Blood Pressure-279@\$100=

Total Cholesterol-281@\$400=

Blood Sugar $\quad-281 @ \$ 300=$

Travelling

TOTAL
AMOUNT (\$)

27.900 .00

$112,400.00$

$84,300,00$

$17,040.00$

$241,640.00$

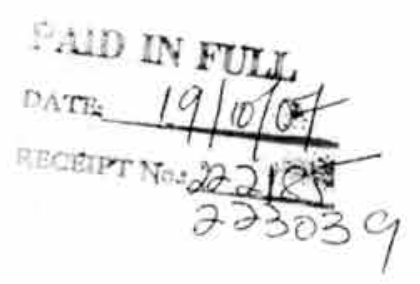




\section{APPENDIX H}

\section{Contract Agreement- Diabetes Association of Jamaica}

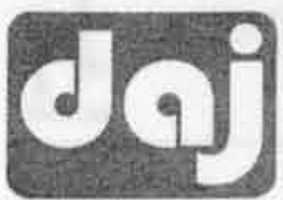

diabetes association of jamaica

Renal Unit (DARU)

1 DOWNER AVENUE, KINGSTON 5 - TELEPHONE: $327-6774$

October 1, 2007

Florida International University

Department of Dietetics \& Nutrition

University Park, HLS 450

11200 S.W. $8^{\text {th }}$ Street

Miami, Fl. 33199

U.S.A.

Dear Sir:

\section{Reference: Sheila Barrett Dissertation Research}

We are pleased to support the above-mentioned in her research, which involves testing Jamaican adolescents, aged 14-19 years for risk factors of Type II Diabetes and Cardiovascular Diseases.

We will be conducting the following test:

300 Hemoglobin A1c Test, using finger prick method, at a cost of US\$10 each, where the total will be US\$3,000.

For further discussion, I can be contacted on 978-5915.

Yours sincerely,

Diabetes Association of Jamaica

Lurline Less (Mrs.)

EXECUTIVE CHAIRMAN
Afmliafed Services:

- Deiwier Plannacy

- Downer Medieal Ciroup

- Kidney Dialygis
Mis Diabesar Cethe than alies rmoldent

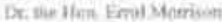

OI $\mathrm{MD}$, PhD FKCH, FACF.

FRSM(I)K,

Prof of Brochemining

4. fintocriating

Frtentive Chairnaa

Jufine Les: OMn:

Tet $\times 76-974.5515$

Fax: $176-930$. HAS

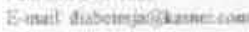

Vice Mhairmas

Estcutive Hirrctar

(Outrab)

Mr Cover Bernard

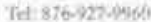

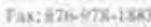

Emat diateteriaflemetcom

Stcretary

Thernis Sื่muh ifMiss

Nouosany Consettaat:

Dr. Knox Hacien

Dr. Ifaese Vernaul

Frof foreand thaino

Itranchol fsland wute

Kinpaton RS Si Aadres

I the

IEI 9276774

4 Swint Strest

Foul ibgin Sigues

Momentilary 5 s Thowe

frt ce?:140

2 Sitiogi Suent

Fon Mtona sa Mar

ict 994.2513

Soust Hied

Port Antonis, Martian:

Tel $352+481$

21 marher Seves

tsimeuth inelism

ThI 9.4-5462

Berh toovte Healiti Caan

Ge Aaniliags fi. Aim

fel 072.7259

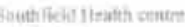

sc Elinaterb

itit theoses

Whielayes liouth Contr

Westnovedanil Heaki Centro

iet: कos.s2in 


\section{CENTRAL MEDICAL LABORATORIES LTD.}

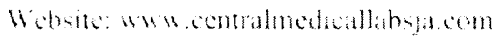

August $31^{\text {st }} 2007$

Miss Sheila Barrett

Florida International University (F.I.U)

Miami

U.S.A.

Dear Miss Barrett,

It was a pleasure hearing from you again and Central Medical Laboratories Ltd. will be awaiting your visit to assist in this very important project you are undertaking.

Listed below is the Updated Quotation for the following tests as requested.

The costs of tests are as follows:

$\begin{array}{lllll}\text { Qty } & \text { Tests } & \text { Unit Price } & \text { Amount Payable } \\ 60 & \begin{array}{l}\text { FBS (FPG) } \\ \text { Lipids } \\ \text { HbA1C }\}\end{array} & \text { JAS2,400.00 } & \text { JAS144,000.00 } \\ & & & \end{array}$

Should you require further information, please do not hesitate to contact the undersigned or Mrs. Denise Ramsay-Thompson, Business Development Manager.

Yours sincerely,

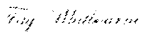

Fay Whitbourne (Dr.)

Consultant Pathologist 


\section{APPENDIX JI}

\section{STUDY PROTOCOL}

7: 00 AM- Six previously randomly selected subjects had their blood taken by venipuncture by the phlebotomist of the Central Medical Laboratory and samples were taken back to Kingston within 4 hours. This activity took place in the sick bay and first thing on data collection day. This group of six were the first to be further examined by the staff of the mobile unit.

7:30 am - Students reported to the sick bay at the school where their names were checked against the list of students made up from the returned consent forms. They were issued an ID card. Students reported to the sick bay as they arrived at school.

Each person conducting an activity wrote their signature on student's ID card as soon as that activity was completed. A school representative (prefect) ushered students from the sick bay in groups of six to the sick bay for blood testing and to then for breakfast.

8:00 am - Students began reporting to the sick bay where the Heart Foundation of Jamaica workers began blood testing. The Heart Foundation worker and worker from Diabetes Association of Jamaica took blood samples by finger prick to measure TC, FBG and $\mathrm{HbAlc}$. The finger was pricked twice to obtain sufficient blood for the three tests.

Students were weighed, had their heights taken, blood pressure measured and waist and hip measured by the Heart Foundation workers and the researcher. Data were entered on form $\mathrm{O}$.

9:00 am First group of students started arriving at a section in the sick bay where they were served a continental breakfast. Students were asked to show their signed ID card indicating that all blood samples (TC, FBG, HbA1c, $\mathrm{BP}$, weight, height, waist and hip circumference) have been taken before they were served a breakfast. Subjects had their ID card checked off after they ate and returned to the sick bay to complete the data collection.

10:00 am After breakfast, students reported back to the sick bay where they were examined by the researcher and school nurse for physical signs of AN. Subjects will answer the demographic and physical activity questionnaires and returned them to the researcher. Researcher checked for completion of questionnaire.

11:00 am- Researcher then conducted the 24 hour recall with each student. 
2:00 pm After completion of 24 hour recall, the ID card was collected and checked to determine if all research activity had been completed, students were then given a monetary incentive of US\$5.00 for their time and effort. 
School

\begin{tabular}{|c|c|c|c|c|c|c|c|c|c|}
\hline $\begin{array}{l}\text { ID } \\
\#\end{array}$ & $\begin{array}{l}\text { Demographic } \\
\text { Data }\end{array}$ & $\begin{array}{l}\text { Physical } \\
\text { Activity }\end{array}$ & \begin{tabular}{|l} 
AN \\
Scale
\end{tabular} & BP & $\mathrm{TC}$ & $\mathrm{HbAlc}$ & FBG & $\begin{array}{l}\text { Food } \\
\text { Record }\end{array}$ & $\begin{array}{l}\text { Amount } \\
\text { Paid (\$) }\end{array}$ \\
\hline \multicolumn{10}{|l|}{1.} \\
\hline \multicolumn{10}{|l|}{2.} \\
\hline \multicolumn{10}{|l|}{3.} \\
\hline \multicolumn{10}{|l|}{4.} \\
\hline \multicolumn{10}{|l|}{5.} \\
\hline \multicolumn{10}{|l|}{6.} \\
\hline \multicolumn{10}{|l|}{7.} \\
\hline \multicolumn{10}{|l|}{8.} \\
\hline \multicolumn{10}{|l|}{9.} \\
\hline \multicolumn{10}{|l|}{10} \\
\hline \multicolumn{10}{|l|}{11} \\
\hline \multicolumn{10}{|l|}{12} \\
\hline \multicolumn{10}{|l|}{13} \\
\hline \multicolumn{10}{|l|}{14} \\
\hline \multicolumn{10}{|l|}{15} \\
\hline \multicolumn{10}{|l|}{16} \\
\hline \multicolumn{10}{|l|}{17} \\
\hline \multicolumn{10}{|l|}{18} \\
\hline \multicolumn{10}{|l|}{19} \\
\hline \multicolumn{10}{|l|}{20} \\
\hline \multicolumn{10}{|l|}{21} \\
\hline \multicolumn{10}{|l|}{22} \\
\hline \multicolumn{10}{|l|}{23} \\
\hline \multicolumn{10}{|l|}{24} \\
\hline \multicolumn{10}{|l|}{25} \\
\hline \multicolumn{10}{|l|}{26} \\
\hline \multicolumn{10}{|l|}{27} \\
\hline \multicolumn{10}{|l|}{28} \\
\hline \multicolumn{10}{|l|}{29} \\
\hline 30 & & & & & & & & & \\
\hline
\end{tabular}

Comments: 


\section{APPENDIX KI}

\section{Permission Letter to Principal for Pilot Study}

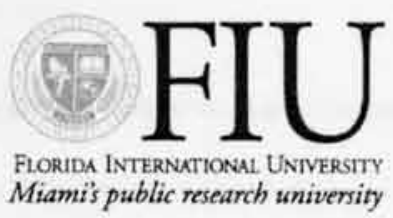

November 16,2006

Dear Sir/Madam,

Your school was randomly selected to participate in a pilot research study conducted by doctoral student, Sheila Barrett and Dr Fatma G. Huffman (major professor) of Florida International University. We are secking your permission to conduct the research study in your institution. This study is in partial fulfillment for a $\mathrm{Ph}$.D, in Dietetics and Nutrition at Florida International University for Miss Barrett.

The study involves screening children ages 14-19 years for risk factors of type 2 diabetes and cardiovascular diseases. Recent research has shown an increasing trend towards type 2 diabetes and cardiovascular diseases in young children and adolescents. I am seeking to conduct a pilot test in your institution prior to conducting the major study which will be conducted in other schools.

If school permission is granted, we will seek further consent from parents, and students to participate in this study. The pilot test involves having 30 randomly selected students from grades $9-11$ to complete a three day food record, a 24 hour recall of all food and beverage consumed, and answering a physical activity and demographic questionnaire. This will take only two hours to complete and will be done during normal school hours.

I am therefore asking your permission to conduct this study in your school and to suggest the name of a teacher who is willing to help with the data collection. Please give your response by checking the attached form and return in the self-addressed, stamped envelope. Your prompt response will be appreciated. Thank you.

Yours truly,

Sheila C. Barrett

(Graduate student and former

lecturer Mico College and U-Tech)
Fatma G. Huffman Ph.D., RD

(major professor)

Robert R. Stempel School of Public Health 


\section{RESPONSE TO REQUEST}

1 give permission to allow the researcher to conduct this pilot study in my school प Yes

Signature

$$
\text { Date }
$$

1 recommend the following teacher to help with data collection

Name of teacher

Telephone

Address:

Email Address:

Name of School: 


\section{IRB Approval- Pilot Study}
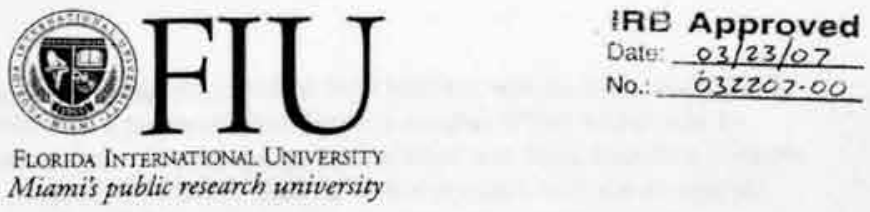

\section{CONSENT/ASSENT TO PARTICIPATE IN A RESEARCH STUDY}

\section{Title: Risk Factors of Type 2 Diabetes and Cardiovascular Diseases Among Jamaican Adolescents.}

We are requesting your consent and your son/daughter's assent to be in a research study. The investigators of this study are Dr Fatma Huffman, Professor of Florida International University, and Sheila Barrett, doctoral student. The study is a pilot study to be undertaken by the researchers. This pilot study will evaluate three different methods of collecting dietary data to assess your child's eating patterns. Approximately 30 other adolescents aged 14-19 years will be selected from a high school in Kingston. The entire study may take up to half an hour of your child's time.

\section{Procedure:}

If you decide to have your child be a part of the study he/she will be asked to;

- Tell the researcher all the foods and drinks that he/she has consumed during the past 24 hours giving details of amounts eaten, and how these foods were prepared. This activity should not take more than 10 minutes of his/her time.

- Keep a record of how much food and drink he/she has eaten over a three-day period, one day should be a week-end day. For this, your child will be given a booklet to record the information and return it to the researcher on day four of the study.

- Complete a demographic questionnaire on his/her past health history, family history of obesity, diabetes and cardiovascular diseases.

- Complete a physical activity check-list. Completion of this checklist can be achieved in less than ten minutes.

\section{Possible Risks}

The risks associated with this study are minimal and require no more than answering some questions. Your child may feel some discomfort by revealing information on how much food he/she eats and his/her physical activity levels. Some of the questions may be embarrassing.

\section{Benefits:}

By participating in this study, you and your child will learn about his/her nutrient intake and activity levels. You and your child will receive the results of the nutrient analysis which can help in planning meals. The research will give us information about the eating patterns of Jamaican adolescents who may be at risk to type 2 diabetes and cardiovascular diseases and help us in planning preventive programs that can be made possible through the schools. There is no cost to you and your child in the study. 
Right to withdraw from the study:

Your child's participation in the study is voluntary and he/she has the right to withdraw from the study at any time.

\section{Confidentiality:}

To protect your child's privacy, all information gathered from him/her will be kept confidential. Your information will be identified by a personal identification number (PIN) which will be assigned to your child by the researcher. No one except Dr Huffman and Miss Barrett will know the name associated with your number. The data collected in this research will not be shared with anyone not directly related to the study unless required by law. The results will be presented as a group in all publications and at the general forum and conferences.

If you or your child would like to know more about the research, you can contact Dr Fatma Huffman at (305) 348-3788 or Miss Sheila Barrett at (305)408-1890. If either of you feel you were mistreated or you have questions about being in the study you may contact Dr. Patricia Price, the Chairperson of the Florida International University Institutional Review Board, at (305) $348-2618$ or (305) 348-2494 .

If you have had all your questions answered to your liking and you would like

(Print Teens Name) to be in the study, sign below.

Signature of ParentGuardian

Date

If you have had all your questions answered to your liking and you would like to be in the study sign below.

Signature of Teen

Date

I have explained the research procedure, subject rights and answered questions asked by the participant. I have offered him/her a copy of this informed consent form.

Signature of Witness

Date 


\title{
IRB Consent Letter- Pilot Study
}

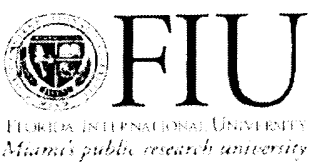

Office of Research Integrity

Rescarch Compliance, MARC 130

\section{MEMORANDUM}

\author{
To: Sheila Barretl \\ CC: Dr. latma Hufman \\ lilis \\ From: Chris Grayson, CIM, Institutional Review Board Coordinator \\ Date: March 23,2007 \\ Proposal Title: Risk Factors of Type 2 Diabetes and Cardiovascular Diseases Anmong \\ Jamaican Adolescents.
}

Approval \# $032207 \% 0$

The Institutional Review Board of Florida International Universily has approved your study for the use of human subjects. Your IRB approval date is March 22, 2007 and this approval will expire on March 22, 2008. As a requirement of IRB approval you are required to

1) Provide immediate written notification to the IRB of:

- Any adultions 10 , or changes in the procedures involving human subjects.

- Fiery serious or unusual or unanticipated adverse event as well as problems with the rights or welfare of the human subjects. Confirmation of receipt of serious Al reports must be made with the IRB office.

2) Utilize copies of the date stamped consent document(s) for the recruitment of subjects and receite ammual renewal of consent documents.

3) Receive annual review and re-approval prior to your expiration date.

Special Comditions: NA

Pleatse mote your approwal number is indicated above. For further information. you may contat the IRB Coordinator by email at irbiacucedtitedu or visit the OSRA ... Human Subjects Website at wiwosiatiucedu. 
Name of School: Subject ID:

\section{INSTRUCTIONS: Circle the number/phrase corresponding to each of the following responses.}

\begin{tabular}{|c|c|c|}
\hline & & ACANTHOSIS NIGRICANS \\
\hline Location & Score & Description \\
\hline Neck & 0 & Absent: not detectable on close inspection \\
\hline & 1 & $\begin{array}{l}\text { Present: clearly present on close visual inspection, not visible } \\
\text { to the casual observer, extent not measurable. }\end{array}$ \\
\hline & 2 & $\begin{array}{l}\text { Mild: limited to the base of skull, does not extend to } \\
\text { the lateral margins of the neck (usually }<3 \text { inches in breadth) }\end{array}$ \\
\hline & 3 & $\begin{array}{l}\text { Moderate: extending to the lateral margins of the neck } \\
\text { (posterior border of the sternocleidomastoid) (usually } 3-6 \text { inches), } \\
\text { should not be visible when the participant is viewed from the } \\
\text { front. }\end{array}$ \\
\hline & 4 & $\begin{array}{l}\text { Severe: extending anteriorly ( }>6 \text { inches), visible when the } \\
\text { participant is viewed from the front. }\end{array}$ \\
\hline Axilla & 0 & Absent: not detectable on close inspection. \\
\hline & 1 & $\begin{array}{l}\text { Present: clearly present on close visual inspection, not } \\
\text { visible to the casual observer, extent not measurable. }\end{array}$ \\
\hline & 2 & $\begin{array}{l}\text { Mild: localized to the central portion of the axilla, may } \\
\text { have gone unnoticed by the participant. }\end{array}$ \\
\hline & 3 & $\begin{array}{l}\text { Moderate: involving entire fossa, but not visible when } \\
\text { the arm is against the participant's side. }\end{array}$ \\
\hline & 4 & $\begin{array}{l}\text { Severe: visible from the front or back in the unclothed } \\
\text { participant when the arm is against the participant's side. }\end{array}$ \\
\hline Neck texture & 0 & Smooth to touch: no differentiation from normal skin to palpation. \\
\hline & 1 & Rough to touch: clearly differentiated from normal skin. \\
\hline & 2 & $\begin{array}{l}\text { Coarseness can be observed visually, portions of the skin } \\
\text { clearly raised above other areas. }\end{array}$ \\
\hline & 3 & $\begin{array}{l}\text { Extremely coarse: "hills and valleys" observable on visual } \\
\text { examination. }\end{array}$ \\
\hline Knuckles & Present & \\
\hline & Absent & \\
\hline Elbows & Present & \\
\hline & Absent & \\
\hline Knees & Present & \\
\hline & Absen & \\
\hline
\end{tabular}




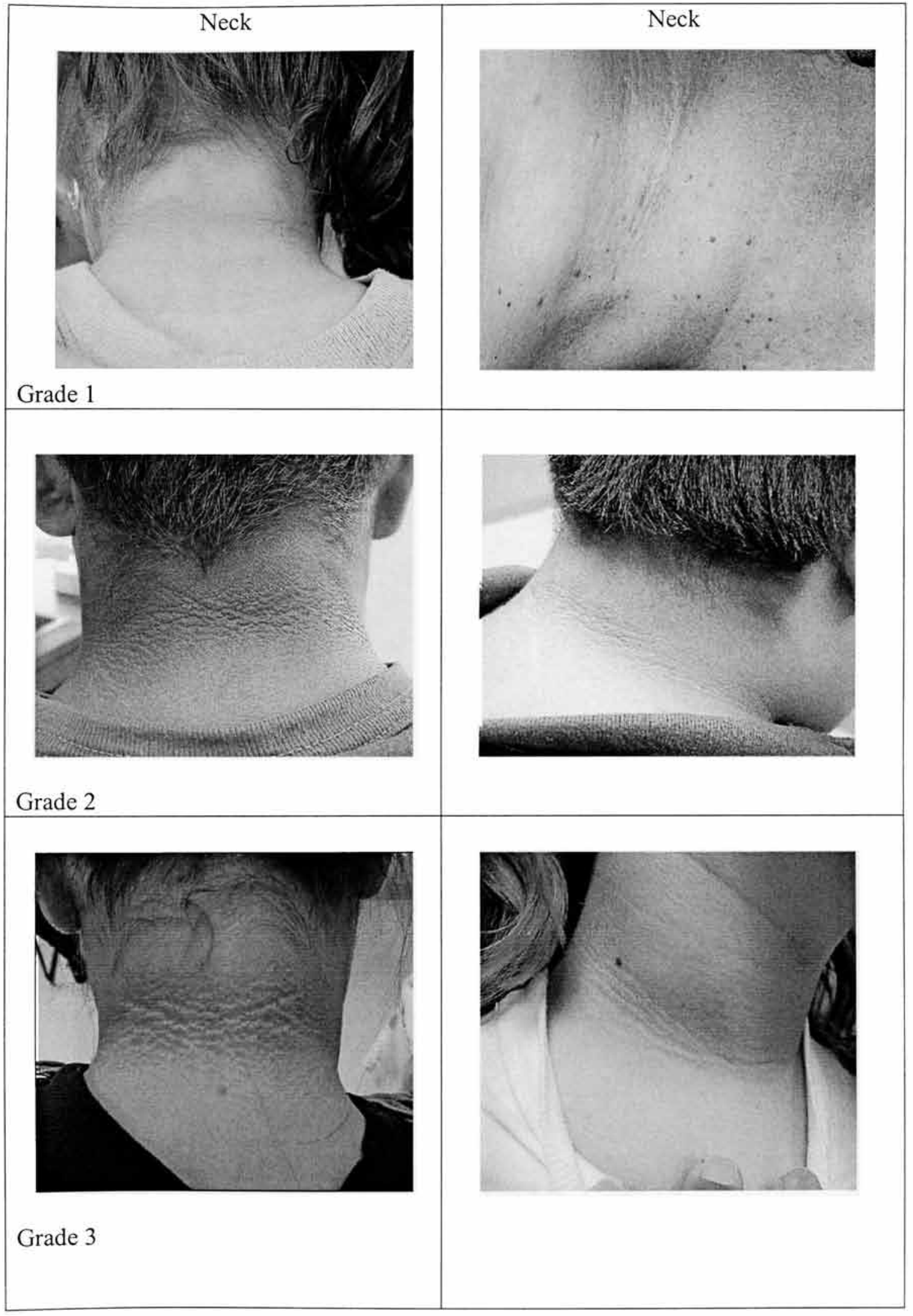




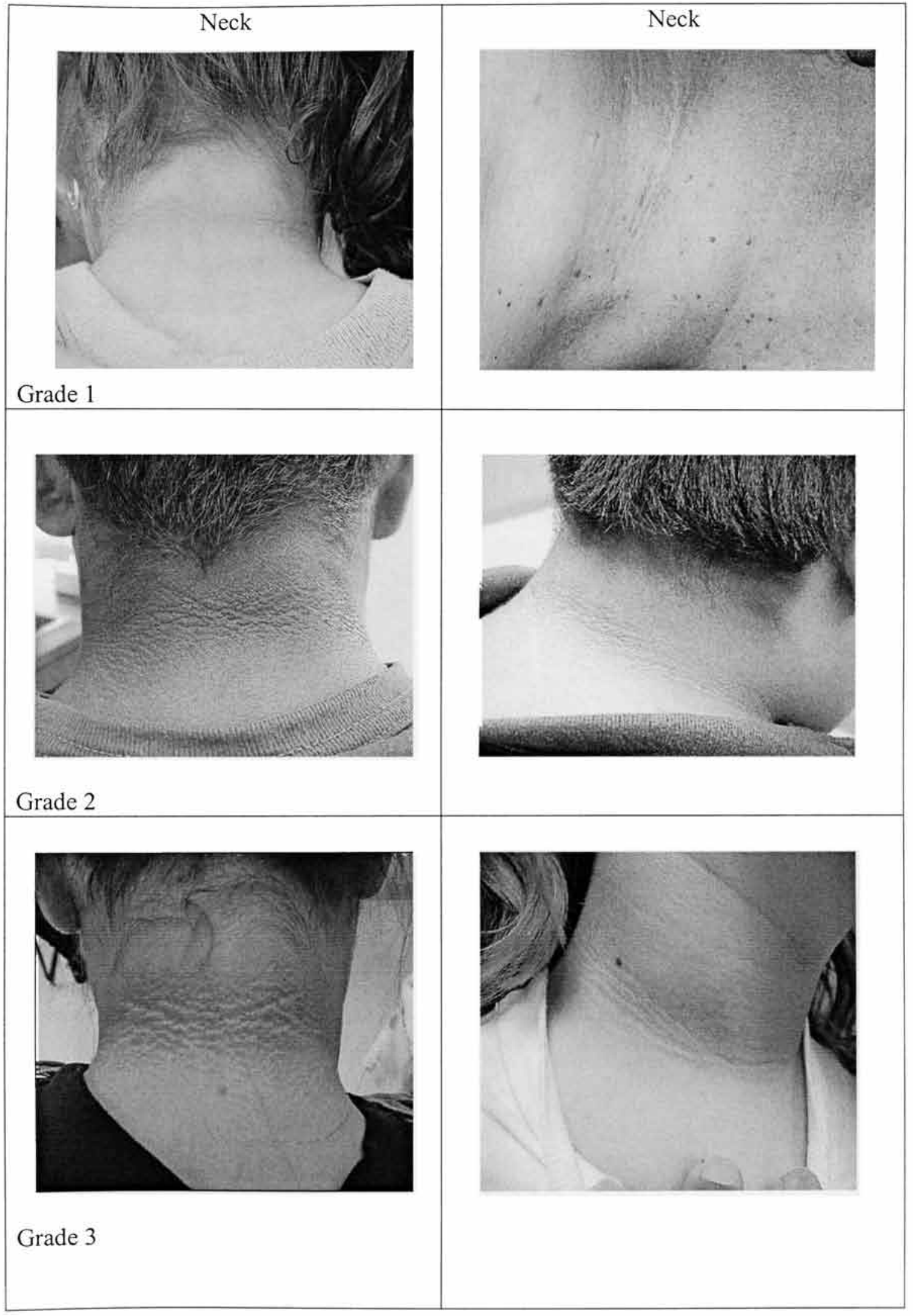




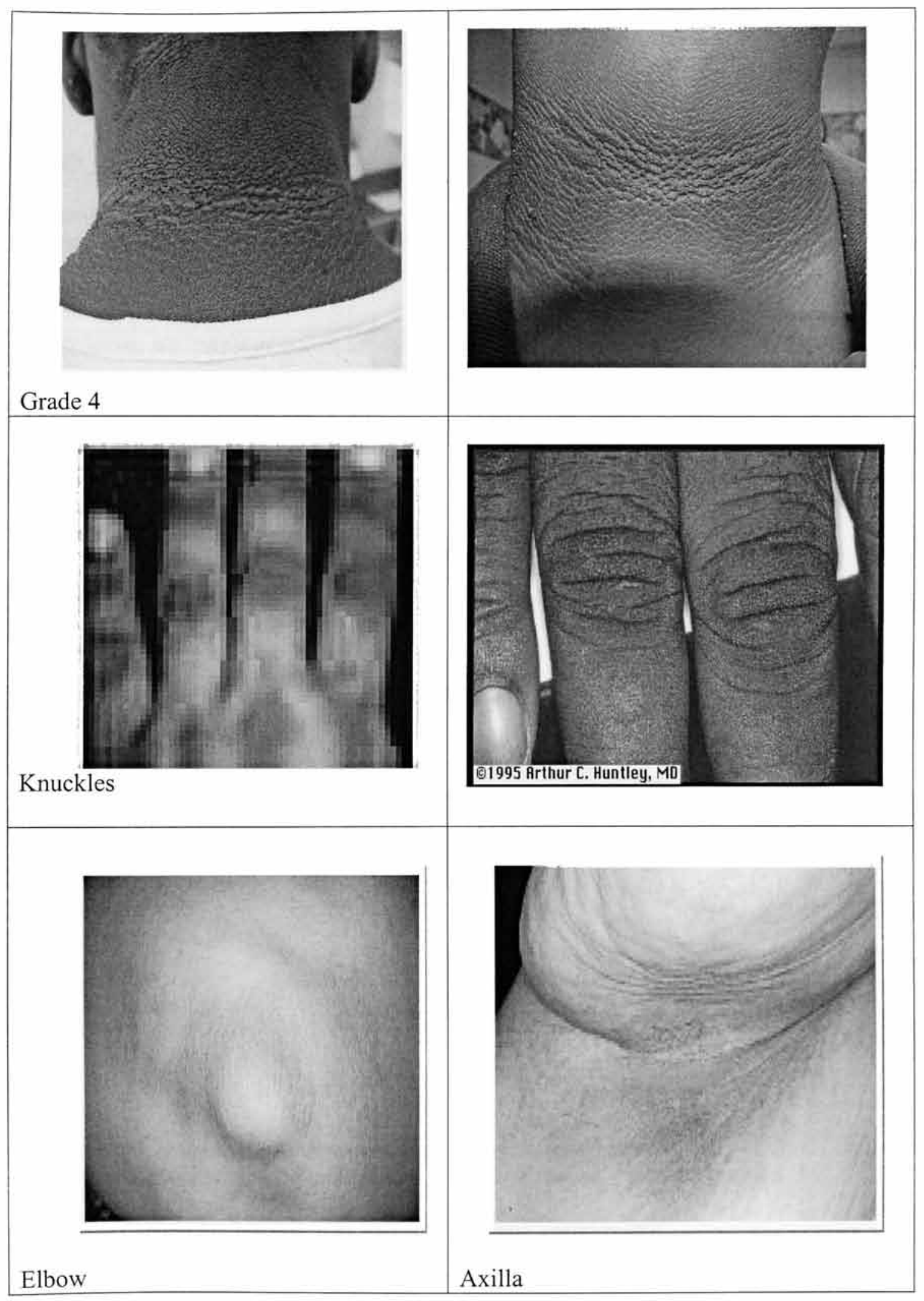




\section{APPENDIX M}

\section{PHYSICAL ACTIVITY QUESTIONNAIRE (TO BE COMPLETED BY STUDENT)}

NAME:

ID:

AGE:

GRADE:

Remember

A. There are no wrong or right answers- this is not a test

B. Please answer all the questions as honestly and accurately as you can- this is very important

\section{PHYSICAL ACTIVITY IN YOUR SPARE TIME}

Have you done any of the following activities in the past 7 days (last week)?

If yes, how many times?

\section{Check Only One Circle Per Box}

Skipping/jump rope

Walking for exercise

Active Games (Tag)

Bicycling

Jogging/running

Swimming laps

Softball

Dance (social, recreational)

Football

Racket sports (tennis, badminton)

Netball

Volleyball

Basketball

Hockey (Street)

Martial Arts (karate, judo)

Cricket

\begin{tabular}{|c|c|c|c|c|}
\hline NO & $\begin{array}{l}\text { If yes, how } \\
\text { often? }\end{array}$ & & & \\
\hline & $1-2$ & $3-4$ & $5-6$ & $>7$ \\
\hline $\mathrm{O}$ & $\mathrm{O}$ & $\mathrm{O}$ & $\mathrm{O}$ & $\mathrm{O}$ \\
\hline $\mathrm{O}$ & $\mathrm{O}$ & $\mathrm{O}$ & $\mathrm{O}$ & $\mathrm{O}$ \\
\hline $\mathrm{O}$ & $\mathrm{O}$ & $\mathrm{O}$ & $\mathrm{O}$ & $\mathrm{O}$ \\
\hline $\mathrm{O}$ & $\mathrm{O}$ & $\mathrm{O}$ & $\mathrm{O}$ & $\mathrm{O}$ \\
\hline $\mathrm{O}$ & $\mathrm{O}$ & $\mathrm{O}$ & $\mathrm{O}$ & $\mathrm{O}$ \\
\hline $\mathrm{O}$ & $\mathrm{O}$ & $\mathrm{O}$ & $\mathrm{O}$ & $\mathrm{O}$ \\
\hline $\mathrm{O}$ & $\mathrm{O}$ & $\mathrm{O}$ & $\mathrm{O}$ & $\mathrm{O}$ \\
\hline $\mathrm{O}$ & $\mathrm{O}$ & $\mathrm{O}$ & $\mathrm{O}$ & $\mathrm{O}$ \\
\hline $\mathrm{O}$ & $\mathrm{O}$ & $\mathrm{O}$ & $\mathrm{O}$ & $\mathrm{O}$ \\
\hline $\mathrm{O}$ & $\mathrm{O}$ & $\mathrm{O}$ & $\mathrm{O}$ & $\mathrm{O}$ \\
\hline $\mathrm{O}$ & $\mathrm{O}$ & $\mathrm{O}$ & $\mathrm{O}$ & $\mathrm{O}$ \\
\hline $\mathrm{O}$ & $\mathrm{O}$ & $\mathrm{O}$ & $\mathrm{O}$ & $\mathrm{O}$ \\
\hline $\mathrm{O}$ & $\mathrm{O}$ & $\mathrm{O}$ & $\mathrm{O}$ & $\mathrm{O}$ \\
\hline $\mathrm{O}$ & $\mathrm{O}$ & $\mathrm{O}$ & $\mathrm{O}$ & $\mathrm{O}$ \\
\hline $\mathrm{O}$ & $\mathrm{O}$ & $\mathrm{O}$ & $\mathrm{O}$ & $\mathrm{O}$ \\
\hline $\mathrm{O}$ & $\mathrm{O}$ & $\mathrm{O}$ & $\mathrm{O}$ & $\mathrm{O}$ \\
\hline $\mathrm{O}$ & $\mathrm{O}$ & $\mathrm{O}$ & $\mathrm{O}$ & $\mathrm{O}$ \\
\hline $\mathrm{O}$ & $\mathrm{O}$ & $\mathrm{O}$ & $\mathrm{O}$ & $\mathrm{O}$ \\
\hline $\mathrm{O}$ & $\mathrm{O}$ & $\mathrm{O}$ & $\mathrm{O}$ & $\mathrm{O}$ \\
\hline $\mathrm{O}$ & $\mathrm{O}$ & $\mathrm{O}$ & $\mathrm{O}$ & $\mathrm{O}$ \\
\hline
\end{tabular}

2. Which ONE of the following describes you best for the last 7 days?

Read ALL FIVE statements before deciding on the one answer that describes you.

O All or most of my free time was spent doing things involving little physical effort.

O I sometimes (1-2 times last week) did physical things in my free time

$\mathrm{O}$ l often (3-4 times last week) did physical things in my free time 
O I quite often (5-6 times last week did physical things in my free time.

$\mathrm{O}$ I very often ( $>7$ times last week) did physical things in my free time.

3. How many days in the past week did you walk or bike to school?

$\begin{array}{ccccc}\text { None } & 1 \text { time } & 2-3 \text { times } & 4 \text { times } & \text { Every day } \\ \mathrm{O} & \mathrm{O} & \mathrm{O} & \mathrm{O} & \mathrm{O}\end{array}$

4. In the last 7 days, during your physical education (PE) classes, how often were you very active (playing hard, running, jumping, throwing)?

$\begin{array}{ccccc}\text { I don't do PE } & \text { Hardly ever } & \text { Sometimes } & \text { Quite Often } & \text { Always } \\ \text { O } & \mathrm{O} & \mathrm{O} & \mathrm{O} & \mathrm{O}\end{array}$

5. In the last 7 days, on how many days RIGHT AFTER SCHOOL, did you do sports, or play games in which you were very active?
None
1 time
2-3 times
4 times
5 times
$\mathrm{O}$
$\mathrm{O}$
$\mathrm{O}$
$\mathrm{O}$
$\mathrm{O}$

6. In the last 7 days, on how many EVENINGS did you play sports, dance, or play games in which you were very active?

$\begin{array}{ccccc}\text { None } & 1 \text { time } & 2-3 \text { times } & 4 \text { times } & 5 \text { times } \\ \mathrm{O} & \mathrm{O} & \mathrm{O} & \mathrm{O} & \mathrm{O}\end{array}$

7. THIS PAST WEEKEND, how many times did you play sports, dance, or play games in which you were very active?

$\begin{array}{ccccc}\text { None } & & & & 6 \text { or more } \\ \text { times }\end{array}$

8. In the last 7 days, what did you do most of the time at RECESS? (check only one)

O Sat down (talking, reading, doing school work).

O Stood around.

O Walked around a little.

O Ran around and played quite a bit.

O Ran and played hard most of the time.

9. In the last 7 days, what did you normally do AT LUNCH (besides eating lunch)? (check only one).

O Sat down (talking, reading, doing school work).

O Stood around.

O Walked around a little.

O Ran around and played quite a bit.

O Ran and played hard most of the time. 


\section{APPENDIX N}

FOOD RECORD- 24-Hour Recall

TO BE COMPLETED BY RESEARCHER AND STUDENT

INSTRUCTIONS FOR FOOD RECORD

(INSIDE COVER OF BOOKLET)

Student ID:

1) Please tell the researcher all the food and drink you consumed for the past 24 hours.

2) Give as much detailed descriptions as possible such as size of item, for example ripe banana (small, medium, large).

3) Tell the researcher the method of preparation such as grilled, fried, baked, boiled....

4) Say where you ate these foods; at home, school (canteen, cafeteria, school vendors), restaurant, fast food joint, road-side vendor).

5) Tell the researcher the exact amounts of each food or drink consumed, use units such as ounces, cups, each, small, medium, large. Refer to diagram for proportions of foods consumed. 


\begin{tabular}{|c|c|c|c|c|}
\hline Meal/Time & Food /Drink & $\begin{array}{l}\text { Description of } \\
\text { food eaten } \\
\text { (fried, grilled, } \\
\text { boiled) }\end{array}$ & $\begin{array}{l}\text { Amounts Eaten } \\
\text { (cups, ozs, each, } \\
\text { small, medium, } \\
\text { large) }\end{array}$ & Location \\
\hline \multirow[t]{4}{*}{ Breakfast } & 1. & & & \\
\hline & 2. & & & \\
\hline & 3. & & & \\
\hline & 4. & & & \\
\hline \multirow[t]{3}{*}{ Snack } & 1. & & & \\
\hline & 2. & & & \\
\hline & 3. & & & \\
\hline \multirow[t]{5}{*}{ Lunch } & 1. & & & \\
\hline & 2. & & & \\
\hline & 3. & & & \\
\hline & 4. & & & \\
\hline & 5. & & & \\
\hline \multirow[t]{5}{*}{ Dinner } & 1. & & & \\
\hline & 2. & & & \\
\hline & 3. & & & \\
\hline & 4. & & & \\
\hline & 5. & & & \\
\hline \multirow[t]{2}{*}{ Snack } & 1. & & & \\
\hline & 2. & & & \\
\hline
\end{tabular}


Name of School: Subject ID:

\begin{tabular}{|c|c|c|c|c|}
\hline \multicolumn{5}{|l|}{ Weight in $\mathrm{Kg}$ : } \\
\hline \multicolumn{5}{|l|}{ Height $\mathrm{cm}$} \\
\hline \multicolumn{5}{|l|}{ BMI } \\
\hline \multicolumn{5}{|l|}{ Percentile BMI } \\
\hline \multicolumn{5}{|l|}{ Waist Circumference $\mathrm{cm}$} \\
\hline \multicolumn{5}{|l|}{ Hip circumference $\mathrm{cm}$} \\
\hline \multicolumn{5}{|l|}{ Waist to Hip Ratio } \\
\hline \multirow[t]{3}{*}{ Blood Pressure $\mathrm{mm} / \mathrm{Hg}$} & Diastolic & \multicolumn{2}{|c|}{ Systolic } & Average \\
\hline & 1) & \multicolumn{2}{|l|}{ 1) } & \\
\hline & 2) & \multicolumn{2}{|l|}{ 2) } & \\
\hline \multirow[t]{2}{*}{ Fasting Blood Glucose $\mathrm{mg} / \mathrm{dL}$} & \multicolumn{3}{|c|}{ Finger prick: FBG } & \\
\hline & \multicolumn{2}{|c|}{ Venipuncture: FPG } & & \\
\hline \multirow[t]{2}{*}{ HbAlc $(\%)$} & \multicolumn{2}{|c|}{ Finger prick: } & & \\
\hline & \multicolumn{2}{|c|}{ Venipuncture: } & & \\
\hline \multirow[t]{2}{*}{ Lipid Profile- TC mg/dL } & \multicolumn{2}{|c|}{ Finger prick: } & \multicolumn{2}{|l|}{$\mathrm{TC}$} \\
\hline & \multicolumn{2}{|c|}{ Venipuncture } & \multicolumn{2}{|l|}{$\mathrm{TC}$} \\
\hline
\end{tabular}




\section{APPENDIX P}

Silhouettes For Measuring Family History of Overweight and Obesity
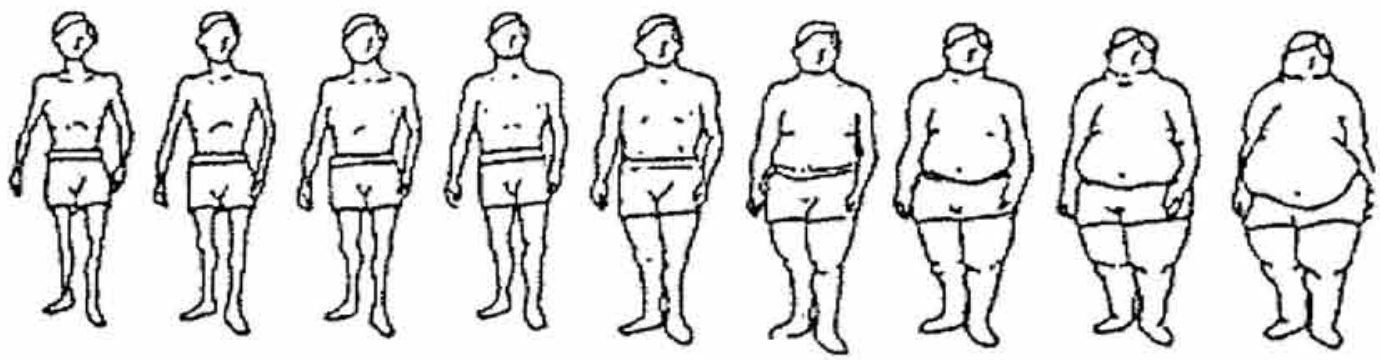

1

2

3

4

5

6

8

9
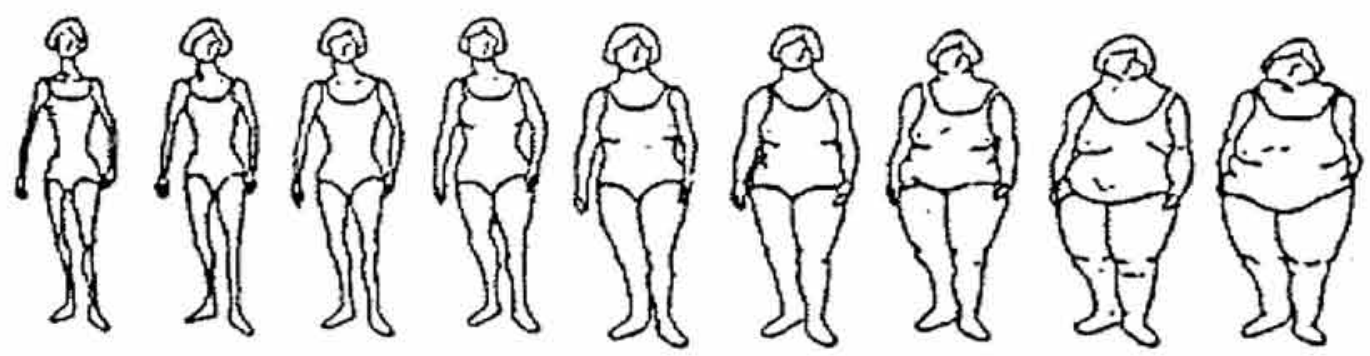

\begin{tabular}{|l|c|c|c|c|c|c|c|c|c|}
\hline Men: & 1 & 2 & 3 & 4 & 5 & 6 & 7 & 8 & 9 \\
\hline \hline BMI: & 19.8 & 21.1 & 22.2 & 23.6 & 25.8 & 28.1 & 31.5 & 35.2 & 41.5 \\
\hline
\end{tabular}

\begin{tabular}{|c|c|c|c|c|c|c|c|c|c|}
\hline Women: & 1 & 2 & 3 & 4 & 5 & 6 & 7 & 8 & 9 \\
\hline BMI: & 18.3 & 19.3 & 20.9 & 23.1 & 26.2 & 29.9 & 34.3 & 38.6 & 45.4 \\
\hline
\end{tabular}

Bulik CM, Wade TD, Heath AC, Martin NG, Stunkard AJ, Eaves $U$. Relating body mass index to figural stimuli: Dopulation-based normative data for Caucasians. Int J Obes Relat Metab Disord, Oct $2001 ; 25(10): 1517-24$ 
Blood Pressure Levels for the $90^{\text {th }}$ and $95^{\text {th }}$ percentile for Boys 1-17 Years

of Age by Percentile of Height

\begin{tabular}{|c|c|c|c|c|c|c|c|c|c|c|c|c|c|c|c|}
\hline \multicolumn{9}{|c|}{ Systolic BP (mmHg) by Percentile of Height } & \multicolumn{7}{|c|}{$\begin{array}{l}\text { Diastolic BP (mmHg) by Percentile } \\
\text { of Height }\end{array}$} \\
\hline Age(Yr) & \%ile & $5 \%$ & $10 \%$ & $25 \%$ & $50 \%$ & $75 \%$ & $90 \%$ & $95 \%$ & $5 \%$ & $10 \%$ & $25 \%$ & $50 \%$ & $75 \%$ & $90 \%$ & $95 \%$ \\
\hline \multirow[t]{2}{*}{1} & $90^{\text {th }}$ & 94 & 95 & 97 & 99 & 101 & 102 & 103 & 49 & 49 & 50 & 51 & 52 & 53 & 54 \\
\hline & $95^{\text {th }}$ & 98 & 99 & 101 & 103 & 105 & 106 & 107 & 54 & 54 & 55 & 56 & 57 & 58 & 58 \\
\hline \multirow[t]{2}{*}{2} & $90^{\text {th }}$ & 98 & 99 & 101 & 103 & 104 & 106 & 107 & 54 & 54 & 55 & 56 & 57 & 58 & 58 \\
\hline & $95^{\text {th }}$ & 101 & 103 & 105 & 107 & 108 & 110 & 110 & 58 & 59 & 60 & 61 & 62 & 63 & 63 \\
\hline \multirow[t]{2}{*}{3} & $90^{\text {th }}$ & 101 & 102 & 103 & 105 & 107 & 109 & 109 & 59 & 59 & 60 & 61 & 62 & 63 & 63 \\
\hline & $95^{\text {th }}$ & 105 & 106 & 107 & 109 & 111 & 112 & 113 & 63 & 63 & 64 & 65 & 66 & 67 & 68 \\
\hline \multirow[t]{2}{*}{4} & $90^{\text {th }}$ & 103 & 104 & 105 & 107 & 109 & 110 & 111 & 63 & 63 & 64 & 65 & 66 & 67 & 67 \\
\hline & $95^{\text {th }}$ & 107 & 108 & 109 & 111 & 113 & 114 & 115 & 67 & 68 & 68 & 69 & 70 & 71 & 72 \\
\hline \multirow[t]{2}{*}{5} & $90^{\text {th }}$ & 104 & 105 & 107 & 109 & 111 & 112 & 113 & 66 & 67 & 68 & 69 & 69 & 70 & 71 \\
\hline & $95^{\text {th }}$ & 108 & 109 & 111 & 113 & 114 & 116 & 117 & 71 & 71 & 72 & 73 & 74 & 75 & 76 \\
\hline \multirow[t]{2}{*}{6} & $90^{\text {th }}$ & 105 & 106 & 108 & 110 & 112 & 113 & 114 & 70 & 70 & 71 & 72 & 73 & 74 & 74 \\
\hline & $95^{\text {th }}$ & 109 & 110 & 112 & 114 & 116 & 117 & 118 & 74 & 75 & 75 & 76 & 77 & 78 & 79 \\
\hline \multirow[t]{2}{*}{7} & $90^{\text {th }}$ & 106 & 107 & 109 & 111 & 113 & 114 & 115 & 72 & 73 & 73 & 74 & 75 & 76 & 77 \\
\hline & $95^{\text {th }}$ & 110 & 111 & 113 & 115 & 117 & 118 & 119 & 77 & 77 & 78 & 79 & 80 & 81 & 81 \\
\hline \multirow[t]{2}{*}{8} & $90^{\text {th }}$ & 108 & 109 & 110 & 112 & 114 & 116 & 116 & 74 & 75 & 75 & 76 & 77 & 78 & 79 \\
\hline & $95^{\text {th }}$ & 112 & 113 & 114 & 116 & 118 & 119 & 120 & 79 & 79 & 80 & 81 & 82 & 83 & 83 \\
\hline \multirow[t]{2}{*}{9} & $90^{\text {th }}$ & 109 & 110 & 112 & 114 & 116 & 117 & 118 & 76 & 76 & 77 & 78 & 79 & 80 & 80 \\
\hline & $95^{\text {th }}$ & 113 & 114 & 116 & 118 & 119 & 121 & 122 & 80 & 81 & 81 & 82 & 83 & 84 & 85 \\
\hline \multirow[t]{2}{*}{10} & $90^{\text {th }}$ & 111 & 112 & 113 & 115 & 117 & 119 & 119 & 77 & 77 & 78 & 79 & 80 & 81 & 81 \\
\hline & $95^{\text {th }}$ & 115 & 116 & 117 & 119 & 121 & 123 & 123 & 81 & 82 & 83 & 83 & 84 & 85 & 86 \\
\hline \multirow[t]{2}{*}{11} & $90^{\text {th }}$ & 113 & 114 & 115 & 117 & 119 & 121 & 121 & 77 & 78 & 79 & 80 & 81 & 81 & 82 \\
\hline & $95^{\text {th }}$ & 117 & 118 & 119 & 121 & 123 & 125 & 125 & 82 & 82 & 83 & 84 & 85 & 86 & 87 \\
\hline \multirow[t]{2}{*}{12} & $90^{\text {th }}$ & 115 & 116 & 118 & 120 & 121 & 123 & 124 & 78 & 78 & 79 & 80 & 81 & 82 & 83 \\
\hline & $95^{\text {th }}$ & 119 & 120 & 122 & 124 & 125 & 127 & 128 & 83 & 83 & 84 & 85 & 86 & 87 & 87 \\
\hline \multirow[t]{2}{*}{13} & $90^{\text {th }}$ & 118 & 119 & 120 & 122 & 124 & 125 & 126 & 78 & 79 & 80 & 81 & 81 & 82 & 83 \\
\hline & $95^{\text {th }}$ & 121 & 122 & 124 & 126 & 128 & 129 & 130 & 83 & 83 & 84 & 85 & 86 & 87 & 88 \\
\hline \multirow[t]{2}{*}{14} & $90^{\text {th }}$ & 120 & 121 & 123 & 125 & 127 & 128 & 129 & 79 & 79 & 80 & 81 & 82 & 83 & 83 \\
\hline & $95^{\text {th }}$ & 124 & 125 & 127 & 129 & 131 & 132 & 133 & 83 & 84 & 85 & 86 & 87 & 87 & 88 \\
\hline \multirow[t]{2}{*}{15} & $90^{\text {th }}$ & 123 & 124 & 126 & 128 & 130 & 131 & 132 & 80 & 80 & 81 & 82 & 83 & 84 & 84 \\
\hline & $95^{\text {th }}$ & 127 & 128 & 130 & 132 & 133 & 135 & 136 & 84 & 85 & 86 & 86 & 87 & 88 & 89 \\
\hline \multirow[t]{2}{*}{16} & $90^{\text {th }}$ & 126 & 127 & 129 & 131 & 132 & 134 & 134 & 81 & 82 & 82 & 83 & 84 & 85 & 86 \\
\hline & $95^{\text {th }}$ & 130 & 131 & 133 & 134 & 136 & 138 & 138 & 86 & 86 & 87 & 88 & 89 & 90 & 90 \\
\hline \multirow[t]{2}{*}{17} & $90^{\text {th }}$ & 128 & 129 & 131 & 133 & 135 & 136 & 137 & 83 & 84 & 85 & 86 & 87 & 87 & 88 \\
\hline & $95^{\text {th }}$ & 132 & 133 & 135 & 137 & 139 & 140 & 141 & 88 & 88 & 89 & 90 & 91 & 92 & 93 \\
\hline
\end{tabular}

Source: Rosner B, Prineas RJ, Loggie JM, Daniels SR: Blood pressure nomograms for children and adolescents, by height, sex, and age, in the United States. J Pediatr 123:87186, 1993. 
Blood Pressure Levels for the $90^{\text {th }}$ and $95^{\text {th }}$ percentile for Girls 1-17 Yearsof Age by

Percentile of Height

\begin{tabular}{|c|c|c|c|c|c|c|c|c|c|c|c|c|c|c|c|}
\hline \multicolumn{9}{|c|}{ Systolic BP (mmHg) by Percentile of Height } & \multicolumn{7}{|c|}{$\begin{array}{l}\text { Diastolic BP }(\mathrm{mmHg}) \text { by Percentile } \\
\text { of Height }\end{array}$} \\
\hline Age(Yr) & \%ile & $5 \%$ & $10 \%$ & $25 \%$ & $50 \%$ & $75 \%$ & $90 \%$ & $95 \%$ & $5 \%$ & $10 \%$ & $25 \%$ & $50 \%$ & $75 \%$ & $90 \%$ & $95 \%$ \\
\hline \multirow[t]{2}{*}{1} & $90^{\text {th }}$ & 98 & 98 & 99 & 101 & 102 & 103 & 104 & 52 & 52 & 53 & 53 & 54 & 55 & 55 \\
\hline & $95^{\text {th }}$ & 101 & 102 & 103 & 104 & 106 & 107 & 108 & 56 & 56 & 57 & 58 & 58 & 59 & 60 \\
\hline \multirow[t]{2}{*}{2} & $90^{\text {th }}$ & 99 & 99 & 101 & 102 & 103 & 104 & 105 & 57 & 57 & 58 & 58 & 59 & 60 & 60 \\
\hline & $95^{\text {th }}$ & 103 & 103 & 104 & 106 & 107 & 108 & 109 & 61 & 61 & 62 & 62 & 63 & 64 & 64 \\
\hline \multirow[t]{2}{*}{3} & $90^{\text {th }}$ & 100 & 101 & 102 & 103 & 104 & 105 & 106 & 61 & 61 & 61 & 62 & 63 & 64 & 64 \\
\hline & $95^{\text {th }}$ & 104 & 104 & 106 & 107 & 108 & 109 & 110 & 65 & 65 & 66 & 66 & 67 & 68 & 68 \\
\hline \multirow[t]{2}{*}{4} & $90^{\text {th }}$ & 101 & 102 & 103 & 104 & 106 & 107 & 108 & 64 & 64 & 65 & 65 & 66 & 67 & 67 \\
\hline & $95^{\text {th }}$ & 105 & 106 & 107 & 108 & 109 & 111 & 111 & 68 & 68 & 69 & 69 & 70 & 71 & 71 \\
\hline \multirow[t]{2}{*}{5} & $90^{\text {th }}$ & 103 & 103 & 105 & 106 & 107 & 108 & 109 & 66 & 67 & 67 & 68 & 69 & 69 & 70 \\
\hline & $95^{\text {th }}$ & 107 & 107 & 108 & 110 & 111 & 112 & 113 & 71 & 71 & 71 & 72 & 73 & 74 & 74 \\
\hline \multirow[t]{2}{*}{6} & $90^{\text {th }}$ & 104 & 105 & 106 & 107 & 109 & 110 & 111 & 69 & 69 & 69 & 70 & 71 & 72 & 72 \\
\hline & $95^{\text {th }}$ & 108 & 109 & 110 & 111 & 113 & 114 & 114 & 73 & 73 & 74 & 74 & 75 & 76 & 76 \\
\hline \multirow[t]{2}{*}{7} & $90^{\text {th }}$ & 106 & 107 & 108 & 109 & 110 & 112 & 112 & 71 & 71 & 71 & 72 & 73 & 74 & 74 \\
\hline & $95^{\text {th }}$ & 110 & 111 & 112 & 113 & 114 & 115 & 116 & 75 & 75 & 75 & 76 & 77 & 78 & 78 \\
\hline \multirow[t]{2}{*}{8} & $90^{\text {th }}$ & 108 & 109 & 110 & 111 & 112 & 114 & 114 & 72 & 72 & 73 & 74 & 74 & 75 & 76 \\
\hline & $95^{\text {th }}$ & 112 & 113 & 114 & 115 & 116 & 117 & 118 & 76 & 77 & 77 & 78 & 79 & 79 & 80 \\
\hline \multirow[t]{2}{*}{9} & $90^{\text {th }}$ & 110 & 111 & 112 & 113 & 114 & 116 & 116 & 74 & 74 & 74 & 75 & 76 & 77 & 77 \\
\hline & $95^{\text {th }}$ & 114 & 115 & 116 & 117 & 118 & 119 & 120 & 78 & 78 & 79 & 79 & 80 & 81 & 81 \\
\hline \multirow[t]{2}{*}{10} & $90^{\text {th }}$ & 112 & 113 & 114 & 115 & 116 & 118 & 118 & 75 & 75 & 76 & 77 & 77 & 78 & 78 \\
\hline & $95^{\text {th }}$ & 116 & 117 & 118 & 119 & 120 & 122 & 122 & 79 & 79 & 80 & 81 & 81 & 82 & 83 \\
\hline \multirow[t]{2}{*}{11} & $90^{\text {th }}$ & 114 & 115 & 116 & 117 & 119 & 120 & 120 & 76 & 77 & 77 & 78 & 79 & 79 & 80 \\
\hline & $95^{\text {th }}$ & 118 & 119 & 120 & 121 & 122 & 124 & 124 & 81 & 81 & 81 & 82 & 83 & 83 & 84 \\
\hline \multirow[t]{2}{*}{12} & $90^{\text {th }}$ & 116 & 117 & 118 & 119 & 121 & 122 & 123 & 78 & 78 & 78 & 79 & 80 & 81 & 81 \\
\hline & $95^{\text {th }}$ & 120 & 121 & 122 & 123 & 125 & 126 & 126 & 82 & 82 & 82 & 83 & 84 & 85 & 85 \\
\hline \multirow[t]{2}{*}{13} & $90^{\text {th }}$ & 118 & 119 & 120 & 121 & 123 & 124 & 124 & 79 & 79 & 79 & 80 & 81 & 82 & 82 \\
\hline & $95^{\text {th }}$ & 122 & 123 & 124 & 125 & 126 & 128 & 128 & 83 & 83 & 84 & 84 & 85 & 86 & 86 \\
\hline \multirow[t]{2}{*}{14} & $90^{\text {th }}$ & 120 & 121 & 122 & 123 & 124 & 125 & 126 & 80 & 80 & 80 & 81 & 82 & 83 & 83 \\
\hline & $95^{\text {th }}$ & 124 & 125 & 126 & 127 & 128 & 129 & 130 & 84 & 84 & 85 & 85 & 86 & 87 & 87 \\
\hline \multirow[t]{2}{*}{15} & $90^{\text {th }}$ & 121 & 122 & 123 & 124 & 126 & 127 & 128 & 80 & 81 & 81 & 82 & 83 & 83 & 84 \\
\hline & $95^{\text {th }}$ & 125 & 126 & 127 & 128 & 130 & 131 & 131 & 85 & 85 & 85 & 86 & 87 & 88 & 88 \\
\hline \multirow[t]{2}{*}{16} & $90^{\text {th }}$ & 122 & 123 & 124 & 125 & 127 & 128 & 129 & 81 & 81 & 82 & 82 & 83 & 84 & 84 \\
\hline & $95^{\text {th }}$ & 123 & 127 & 128 & 129 & 130 & 132 & 132 & 85 & 85 & 85 & 87 & 87 & 88 & 88 \\
\hline \multirow[t]{2}{*}{17} & $90^{\text {th }}$ & 123 & 123 & 124 & 126 & 127 & 128 & 129 & 81 & 81 & 82 & 83 & 83 & 84 & 85 \\
\hline & $95^{\text {th }}$ & 127 & 127 & 128 & 130 & 131 & 132 & 133 & 85 & 86 & 86 & 87 & 88 & 88 & 89 \\
\hline
\end{tabular}

Source: Rosner B, Prineas RJ, Loggie JM, Daniels SR: Blood pressure nomograms for children and adolescents, by height, sex, and age, in the United States. J Pediatr 123:87186, 1993. 


\section{APPENDIX S}

Comparison of Subject's BMI with Parents' BMI

\begin{tabular}{|c|c|c|c|c|c|c|}
\hline \multirow[t]{2}{*}{ BMI Categories } & \multicolumn{2}{|l|}{ Subjects BMI } & \multicolumn{2}{|l|}{ Mothers BMI } & \multicolumn{2}{|l|}{ Fathers BMI } \\
\hline & $\mathrm{N}(\%)$ & Mean \pm SD & $N(\%)$ & Mean \pm SD & $\mathrm{N}(\%)$ & Mean \pm SD \\
\hline Overall BMI & 276 & $23.76 \pm 7.72$ & 267 & $24.72 \pm 6.55$ & 255 & $23.14 \pm 7.40$ \\
\hline Underweight $(\mathrm{BMI}<18.5)$ & $68(24.6 \%)$ & $16.72 \pm 1.26$ & $11(4.0 \%)$ & $18.3 \pm .0$ & 0 & 0 \\
\hline Normal (BMI18.0-24.9) & $118(42.4 \%)$ & $20.91 \pm 1.75$ & $116(42.0 \%)$ & $22.06 \pm 1.37$ & $134(48.6 \%)$ & $22.47 \pm 1.22$ \\
\hline Overweight (BMI 25.0-29.9) & $41(14.9 \%)$ & $27.32 \pm 1.38$ & $109(39.5 \%)$ & $27.54 \pm 1.80$ & $91(33.0 \%)$ & $26.56 \pm 1.08$ \\
\hline $\begin{array}{l}\text { Obesity Class I (BMI 30.0- } \\
34.9 \text { ) }\end{array}$ & $21(7.2 \%)$ & $32.33 \pm 1.68$ & $24(8.7 \%)$ & $34.3 \pm .0$ & $27(9.8 \%)$ & $31.63 \pm 0.71$ \\
\hline $\begin{array}{l}\text { Obesity Class II (BMI 35.0- } \\
39.9 \text { ) }\end{array}$ & $18(6.9 \%)$ & $36.83 \pm 0.98$ & $6(2.2 \%)$ & $37.3 \pm 2.0$ & $3(1.1 \%)$ & $35.4 \pm 0.34$ \\
\hline Obesity Class III (BMI > 40) & $11(4.0 \%)$ & $46.21 \pm 8.43$ & $1(0.4 \%)$ & $45.4 \pm .0$ & 0 & 0 \\
\hline Missing & 0 & & $9(3.3 \%)$ & & $21(7.6 \%)$ & \\
\hline
\end{tabular}




\section{APPENDIX T}

Relationships Between Select Macronutrient Intakes and Risk Factors of T2DM and CVDS

\begin{tabular}{|c|c|c|c|c|c|c|}
\hline \multirow[t]{2}{*}{$\begin{array}{l}\text { Risk } \\
\text { Factors }\end{array}$} & \multicolumn{2}{|c|}{$\begin{array}{l}\text { Total Carbohydrate } \\
\text { g) } \\
\quad(n=273)\end{array}$} & \multicolumn{2}{|c|}{$\begin{array}{l}\text { Total fats }(\mathrm{g}) \\
\quad(\mathrm{n}=272)\end{array}$} & \multicolumn{2}{|c|}{$\begin{array}{c}\text { Total kcals } \\
(\mathrm{n}=273)\end{array}$} \\
\hline & $\mathrm{r}$ & $P$ value & $\mathrm{r}$ & $\mathrm{P}$ value & $\mathrm{r}$ & $\mathrm{P}$ value \\
\hline High BMI & -.17 & .004 & -.16 & .006 & -.17 & .004 \\
\hline High WC & -.14 & .020 & -.18 & .003 & -.15 & .011 \\
\hline Acanthosis Nigricans & -.15 & .013 & -.13 & .036 & -.13 & .029 \\
\hline Family history of T2DN & -.06 & .330 & -.06 & .333 & -.16 & .008 \\
\hline Total \# of risk factors & -.12 & .039 & -.08 & .169 & -.11 & .073 \\
\hline
\end{tabular}




\section{APPENDIX U}

Table 19

Relationships between Complex and Refined Carbohydrate Intakes and Risk Factors of $T 2 D M$ and $C V D S$

\begin{tabular}{lrrrrrrr}
\hline Risk factors & \multicolumn{2}{c}{$\begin{array}{c}\text { Starch }(\mathrm{g}) \\
(\mathrm{n}=273)\end{array}$} & \multicolumn{2}{c}{$\begin{array}{c}\text { Sugar }(\mathrm{g}) \\
(\mathrm{n}=268)\end{array}$} & \multicolumn{2}{c}{$\begin{array}{c}\text { Fiber }(\mathrm{g}) \\
(\mathrm{n}=270)\end{array}$} \\
\cline { 2 - 8 } & $\mathrm{r}$ & $\mathrm{P}$ value & $\mathrm{r}$ & P value & $\mathrm{r}$ & P value \\
\hline BMI & -.14 & .017 & -.14 & .020 & -.15 & .015 \\
Waist Circumference & -.12 & .045 & -.11 & .080 & -.15 & .012 \\
Physical Activity & .13 & .026 & -.13 & .029 & .08 & .161 \\
Family History of Obesity & -.12 & .056 & -.03 & .631 & -.17 & .005 \\
Acanthosis Nigricans & -.15 & .011 & 0.05 & .388 & -.18 & $.003^{*}$ \\
Total \# of risk factors & -.13 & .030 & -.06 & .339 & -.14 & .017 \\
\hline F Significant ung
\end{tabular}

* Significant using the Holm's Sequential Bonferroni Method overall $\alpha=.05$ for each macronutrient variable. 


\section{APPENDIX V}

Table 20

Relationships Between Types of Fats and Risk Factors of T2DM and CVDS

\begin{tabular}{lcccccr}
\hline Risk Factors & $\begin{array}{c}\text { Saturated Fats } \\
(\mathrm{g}) \\
(\mathrm{n}=273)\end{array}$ & $\begin{array}{c}\text { Monounsaturated Fats } \\
(\mathrm{g}) \\
(\mathrm{n}=273)\end{array}$ & \multicolumn{2}{c}{$\begin{array}{c}\text { Polyunsaturated } \\
\text { Fats }(\mathrm{g}) \\
(\mathrm{n}=273)\end{array}$} \\
\cline { 2 - 7 } & $\mathrm{r}$ & $\mathrm{P}$ value & $\mathrm{r}$ & $\mathrm{P}$ value & $\mathrm{r}$ & P value \\
\hline HbA1c & .04 & .517 & .13 & .032 & .13 & .031 \\
BMI & -.10 & .094 & -.12 & .051 & -.12 & .043 \\
WC & -.16 & .007 & -.15 & .013 & -.13 & .028 \\
AN & -.17 & .006 & -.17 & .005 & -.15 & .011 \\
\hline
\end{tabular}

No significance found using the Holm's Sequential Bonferroni Method overall $\alpha=.05$ for each macronutrient variable and the 13 risk factors. 


\section{APPENDIX W}

Boxplots Comparing Finger prick and Venous Measurements of FBG

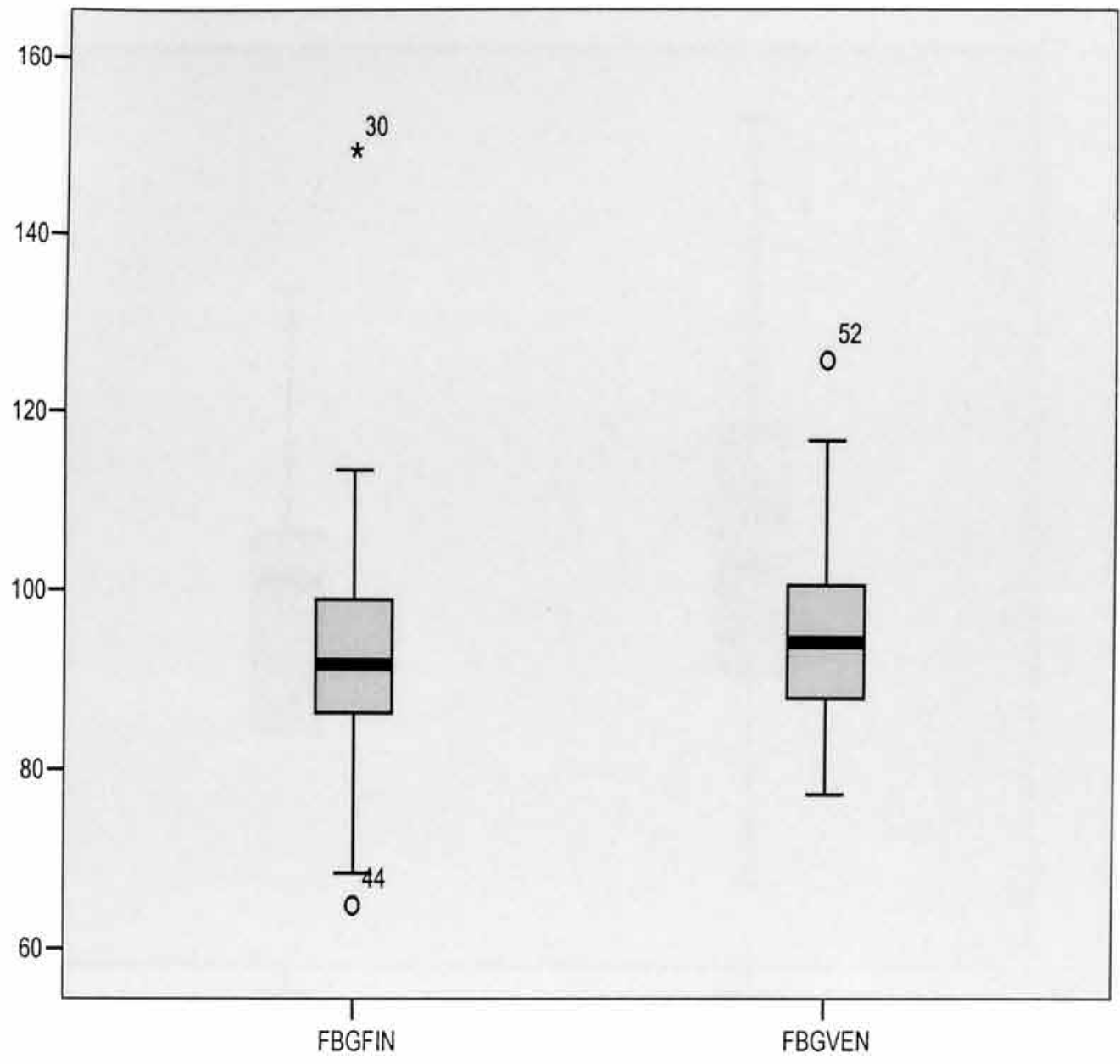




\section{APPENDIX X}

Boxplots Comparing Finger prick and Venous Measurements of TC

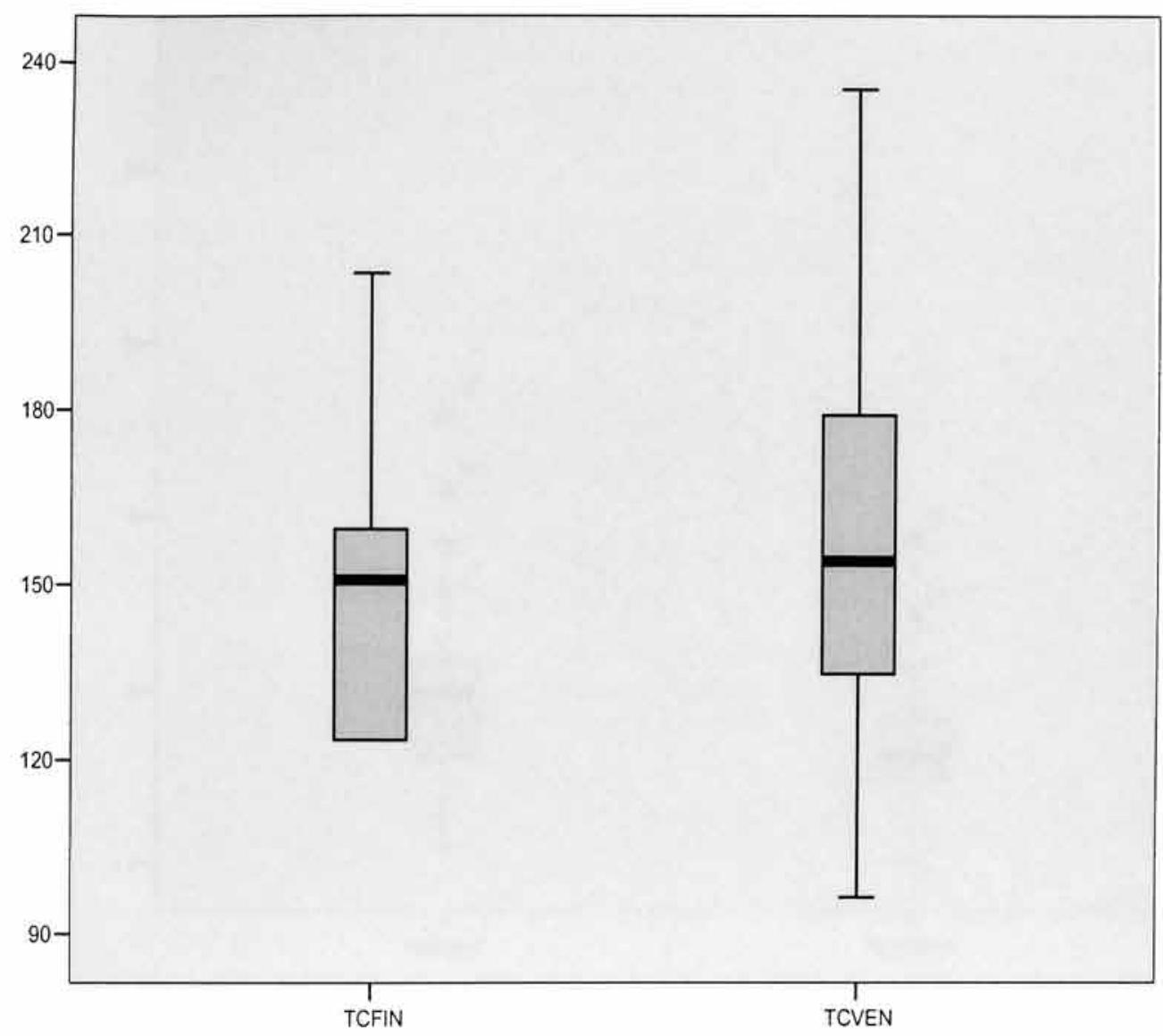




\section{APPENDIX Y}

Boxplots Comparing Finger prick and Venous Measurements of $\mathrm{HbAlc}$

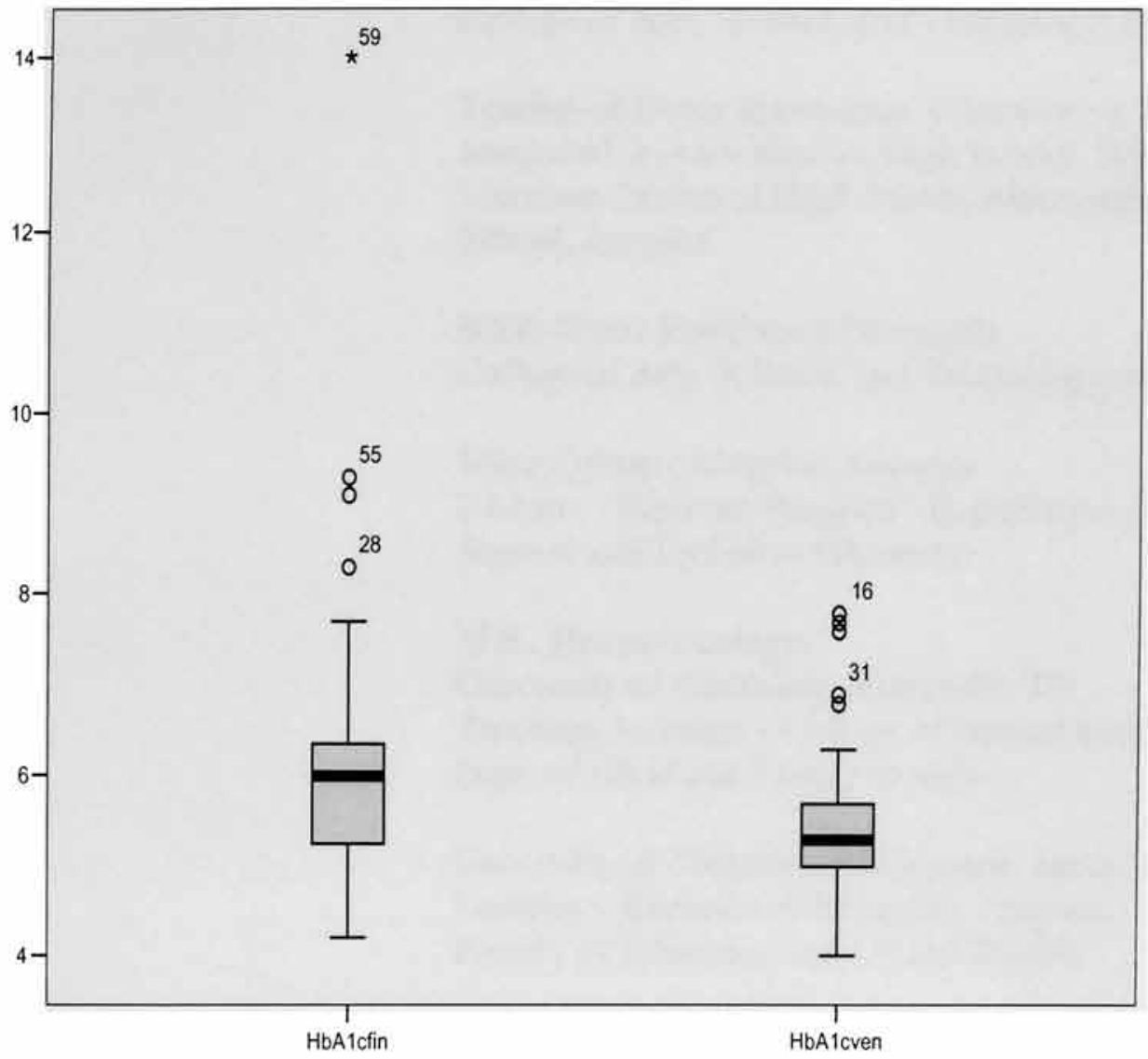




\section{VITA}

\section{SHEILA C. BARRETT}

1982

$1982-90$

1990

1990-94

1996-2001

1994-1996

2001

August 2002- present
Technical Teachers' Diploma Home Economics College of Arts, Science, and Technology, Jamaica

Teacher of Home Economics, Chemistry and Integrated Science Rusea's High School, Herbert Morrison Technical High School, Manning's High School, Jamaica.

B.Ed. Home Economics Education College of Arts, Science, and Technology, Jamaica

Mico College, Kingston, Jamaica Lecturer - Diploma Program - Department of Science and Technical Education

M.S., Human Ecology, University of Tennessee, Knoxville, TN Teaching Assistant - College of Human Ecology, Dept. of Child and Family Studies

University of Technology, Kingston, Jamaica Lecturer - Bachelor of Education Program -Faculty of Education and Liberal Studies

Doctoral Candidate Florida International University, Miami, Florida Teaching Assistant - Robert Stempel College of Public Health and Social Work; Department of Dietetics \& Nutrition

\section{Awards and Memberships}

Dissertation Year Fellowship (2009) Florida International University, Latin American Scholarship Program for American Universities (LASPAU) Full-bright Scholarship 1994-1996; Member of Phi Kappa Phi, Kappa Omnicron Nu, Phi Beta Delta, and Delta Epsilon Iota for academic excellence; Jamaican Home Economics Association, Caribbean Association of Home Economists and International Federation of Home Economists.

\section{Positions Held}

Vice-President and President of the Association of Graduate Students in Dietetics and Nutrition, Florida International University 2003-2005, Secretary and Chairman - Home Economics Board of Studies, Joint Board of Teacher Education, University of the West Indies 1996-2001 and 2000-2001. 
To: Dean Vish Prasad

College of Engineering

This dissertation, written by Andres Barrios, and entitled Nonlinear Quantum Transport in Low-Dimensional Electronic Devices, having been approved in respect to style and content, is referred to you for judgment.

We have read this dissertation and recommend that it be approved.
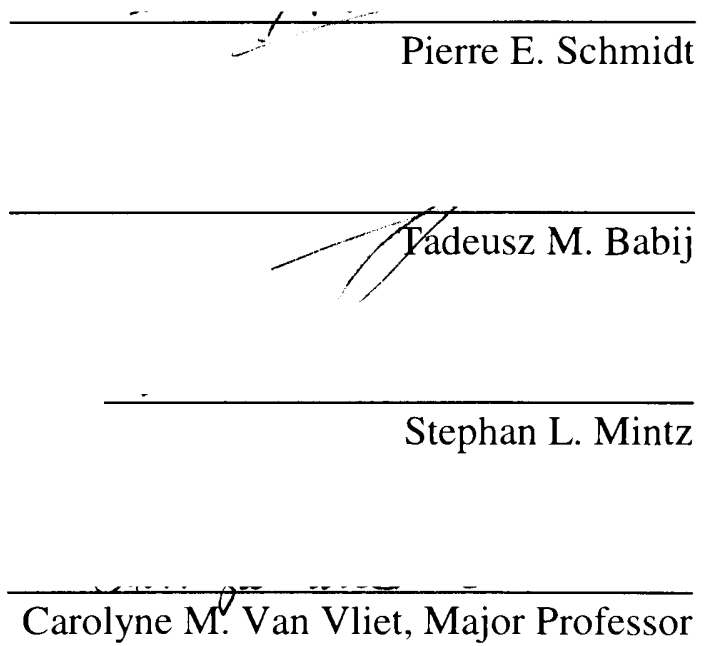

Date of Defense: April 4, 2002

The dissertation of Andres Barrios is approved.

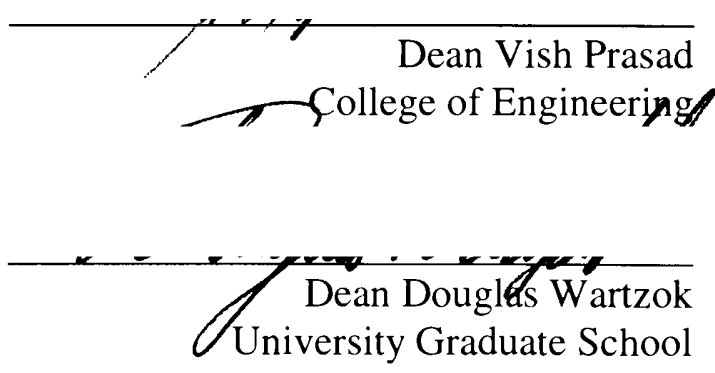

Florida International University, 2002 